\title{
WestVirginiaUniversity
}

THE RESEARCH REPOSITORY @ WVU

Graduate Theses, Dissertations, and Problem Reports

2016

\section{Modeling of magnetoelectric composite structures}

Tomas Ignacio Muchenik Cena

Follow this and additional works at: https://researchrepository.wvu.edu/etd

\section{Recommended Citation}

Muchenik Cena, Tomas Ignacio, "Modeling of magnetoelectric composite structures" (2016). Graduate Theses, Dissertations, and Problem Reports. 7114.

https://researchrepository.wvu.edu/etd/7114

This Dissertation is protected by copyright and/or related rights. It has been brought to you by the The Research Repository @ WVU with permission from the rights-holder(s). You are free to use this Dissertation in any way that is permitted by the copyright and related rights legislation that applies to your use. For other uses you must obtain permission from the rights-holder(s) directly, unless additional rights are indicated by a Creative Commons license in the record and/ or on the work itself. This Dissertation has been accepted for inclusion in WVU Graduate Theses, Dissertations, and Problem Reports collection by an authorized administrator of The Research Repository @ WVU.

For more information, please contact researchrepository@mail.wvu.edu. 


\title{
Modeling of magnetoelectric composite structures
}

\section{Tomás Ignacio Muchenik Ceña}

Dissertation submitted

to the College of Engineering and Mineral Resources

at West Virginia University

in partial fulfillment of the requirements for the degree of

Doctor of Philosophy in

Mechanical Engineering

\author{
Ever J. Barbero, Ph.D., Chair \\ Fritz Campo, Ph.D., \\ Victor Mucino, Ph.D., \\ Xueyan Song, Ph.D., \\ Eduardo M. Sosa, Ph.D.
}

Department of Mechanical and Aerospace Engineering

Morgantown, West Virginia

May 3, 2016

Keywords: Composite, Magnetoelectric, Magnetostrictive, Modeling, Piezoelectric, Piezomagnetic, Demagnetization, Eshelby, Particulate Composite, Micromechanics, Green's function, Dilute approximation, Mori-Tanaka mean field theory Copyright 2016 Tomás Ignacio Muchenik Ceña 


\section{Abstract}

\section{Modeling of magnetoelectric composite structures \\ Tomás Ignacio Muchenik Ceña}

Novel models to predict magnetoelectric (ME) properties of composites made of piezoelectric $(\mathrm{PE})$ and piezomagnetic $(\mathrm{PM})$ phases is proposed. Two different composite arrangements are used: laminate and particulate. ME properties for laminate arrangement are obtained by applying the multiphysics equations for all four possible laminate configurations (TT, LT, TL, and LL), with appropriate boundary conditions. Closed form, explicit formulas are derived for the calculation of the ME charge and voltage coefficients as a function of material properties of both phases and PM volume fraction. A new coefficient, the ME coupling factor, is proposed in order to assess the conversion of magnetic work into electric work. The predicted ME voltage coefficient is in agreement with previous work and experimental data. A new approach is proposed to take into account the conductivity of the PM phase, resulting in calculated ME charge coefficients within 30\% of experimental data. The voltage, current, and electric power generated by unit of magnetic field applied to the composite define the intrinsic voltage, current, and power conversion factors. Since the PM phase of the composite has a higher magnetic permeability than the surrounding medium, a far filed magnetic field is not fully utilized due to demagnetization. Thus, novel explicit equations are developed here to calculate the extrinsic voltage, current, and power conversion factors accounting for demagnetization. The proposed formulation is applied to various materials 
and geometries to illustrate the process of material and device-geometry selection leading to an optimum design. The magnetoelectric (ME) properties of particulate composites are calculated using Eshelby theory and two homogenization techniques: dilute approximation and Mori-Tanaka mean field theory. A method that allows the calculation of all ME properties under any boundary conditions is proposed. These boundary conditions are dictated by the experimental configuration, e.g. films on a substrate, free-standing composites, etc. Predictions are compared with calculations reported by Harshe et al. and Nan et al., and good correlation is obtained with those, but to achieve good correlation with experimental data, the conductivity of the piezomagnetic (PM) phase must be taken into account, and a method is proposed to that effect. Percolated composites do not have any piezoelectric (PE) or ME properties because the charge leaks through the conductive PM phase. The experimental parameters that influence the percolation threshold are discussed and the best particulate composite design is proposed. Unlike previous models that did not account for conductivity, correlation between the proposed model and experimental data is much better. 


\section{Acknowledgments}

I am grateful to my committee chair, Prof. Ever Barbero, for making my graduate studies a great academic and personal experience. Having the opportunity to conduct research with Prof. Barbero allowed me to write a book chapter, several peer reviewed papers, and present my work in several conferences, while having a very pleasant time. As my mentor, he was always very supportive and encouraged my ideas while providing excellent guidance.

I would like to thank my committee members; Dr. Fritz Campo, Prof. Victor Mucino, Prof. Xueyan Song, and Prof. Eduardo M. Sosa. They gave me very valuable advice and support to accomplish my Ph.D. dissertation. I would especially like to thank Prof. Mucino and Prof. Jacky Prucz for helping me secure a stipend from the department through a graduate teaching assistantship, which was a very productive and enriching experience.

I am also grateful for Lisa Kogan, Jonathan Taub, Ozcan Ozmen, Anirudh Tirus, Manish Aggarwal, and Derrick Banerjee for helping me in my research and for making this experience a playful, intellectual adventure as much as it was productive.

My thanks are extended to all who, directly or indirectly, have given their helping hand in this venture. Personally, I would like to thank my loved ones for their support, my friends for the enjoyable times and especially thank my family for supporting me spiritually throughout my life. 


\section{Contents}

Acknowledgments $\quad$ iv

1 Introduction $\quad 1$

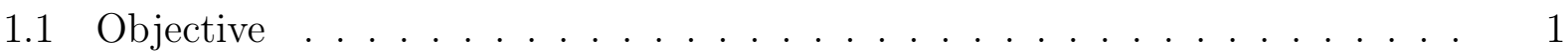

1.2 Magnetoelectric effect . . . . . . . . . . . . . . . . . 2

1.3 Historical review . . . . . . . . . . . . . . . . . . . . . . 4

1.4 ME effect in composite materials . . . . . . . . . . . . . . . . . . 6

1.4.1 Discovery of ME effect in composite materials . . . . . . . . . . 8

1.4.2 ME composites made by ceramic sintering . . . . . . . . . . . . 9

1.4.3 Laminate composites . . . . . . . . . . . . . . . 16

1.4.4 Resonance frequency . . . . . . . . . . . . . . . . . . 23

1.5 Thin films ME composites . . . . . . . . . . . . . . . . . . 23

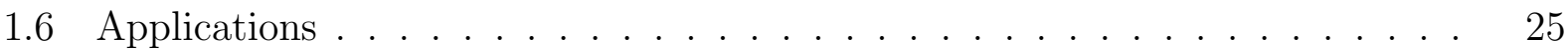

1.6.1 Magnetic sensors ....................... 25

1.6.2 Magnetoelectric harvesters . . . . . . . . . . . . . 25

1.7 Magnetoelectric modeling . . . . . . . . . . . . . . 36

1.7.1 Analytical models . . . . . . . . . . . . . . . . . 36

1.7.2 Numerical models . . . . . . . . . . . . . . . . . . . 42

2 Charge, voltage, and work-conversion formulas for magnetoelectric laminated composites 
2.1 Introduction . . . . . . . . . . . . . . . . . 46

2.2 Model description . . . . . . . . . . . . . . . . . . . . . . . . . 49

2.2.1 Transverse magnetization and polarization $(\mathrm{TT}) \ldots \ldots$. . . . . . . 53

2.2.2 Longitudinal magnetization and transverse polarization (LT) . . . . . 60

2.2 .3 TL configuration . . . . . . . . . . . . . . 66

2.2.4 LL configuration . . . . . . . . . . . . . . . . 67

2.3 ME coupling Factor . . . . . . . . . . . . . . . . . . . . . . 68

2.4 Demagnetizing Factor . . . . . . . . . . . . . . . . . 72

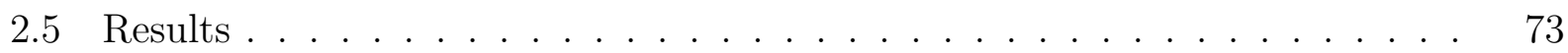

2.6 Conclusions . . . . . . . . . . . . . . . . . . . . 77

3 Design selection using extrinsic charge, voltage, and work-conversion factors for laminated magnetoelectric composites $\quad 80$

3.1 Introduction . . . . . . . . . . . . . . . . . 81

3.2 Materials and constitutive equations . . . . . . . . . . . . . 82

3.3 Intrinsic properties . . . . . . . . . . . . . . . . 86

3.3.1 TT configuration ..................... 87

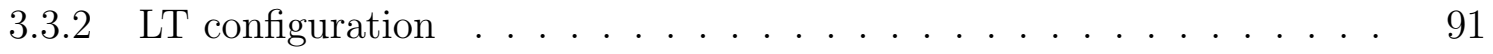

3.3.3 TL configuration ......................... 94

3.3.4 LL configuration . . . . . . . . . . . . . . . . . 94

3.3.5 Summary of intrinsic properties . . . . . . . . . . . . . . 97

3.4 Extrinsic ME properties . . . . . . . . . . . . . . . . . . . 99

3.4.1 Extrinsic ME voltage coefficient . . . . . . . . . . . . . . . . 102

3.4.2 Extrinsic ME charge coefficient . . . . . . . . . . . . . . . . . 112

3.4.3 Extrinsic ME coupling factor . . . . . . . . . . . . 116

3.5 Conclusions . . . . . . . . . . . . . . . . . . . . 118

4 Prediction of magnetoelectric coefficients for particulate composites $\quad 121$ 
4.1 Introduction . . . . . . . . . . . . . . . . . . . 122

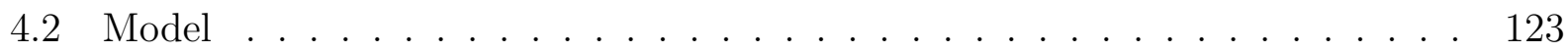

4.3 Magnetoelectric constants . . . . . . . . . . . . . . . . . 128

4.4 Percolation threshold . . . . . . . . . . . . . . . . . . . . . . 130

4.5 Validation . . . . . . . . . . . . . . . . . . . . 132

4.5.1 Non-conductive PM matrix . . . . . . . . . . . . . . 136

4.5.2 Conductive PM matrix . . . . . . . . . . . . . . . 137

4.5.3 PE matrix with dispersed PM phase . . . . . . . . . . . . . . 137

4.6 Results . . . . . . . . . . . . . . . . . . . . 139

4.7 Conclusions . . . . . . . . . . . . . . . . . . . . . . . . 144

5 Conclusions $\quad 145$

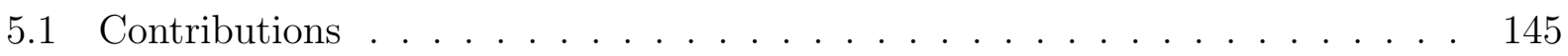

5.2 Future work . . . . . . . . . . . . . . . . . . . 148 


\section{Chapter 1}

\section{Introduction}

\section{$1.1 \quad$ Objective}

In the present work, multiphysics models are presented to predict the ME properties for both laminate and particulate composites. In the case of ME laminate composites the current models in literature deviate from experimental results because they do not consider the high conductivity of the PM phase. The objective of this work is to properly account for this and obtain good correlation with experimental data. The ME properties calculated in literature do not address the applications for energy harvesting. The objective of this work is to develop a ME coupling factor to calculate the electric power that can be harvested by a ME composite when exposed to magnetic fields, and vice versa. If the magnetic field is not applied directly into the boundaries of the composite, a demagnetizing effect will reduce the applied magnetic field. The objective of this work is to account for this demagnetizing effect and define the extrinsic ME properties. The final objective is to design and select the optimum material combination from the available PM and PE phases, optimum volume fraction and optimal configuration, as a function of the desired device aspect ratio.

In the case of ME particulate composite, the implications of the conductive PM phase are more important. For example, the fabricated ME particle composites, reported in the 
bibliography, that have PM phase as a matrix do not show any ME output, but the models in literature predict ME output. Furthermore, these ME properties will also vanish in the case of percolated PM phase. The objective of this work is to be able to account for the conductivity of the PM phase to be in agreement with reported experimental data. The objective of this work is to also account for the effect that different mechanical boundary conditions (free standing, thin film) have in the ME properties.

\subsection{Magnetoelectric effect}

There are different ferroic orders, e.g., ferroelectric, ferromagnetic, ferroelastic. The materials in which different ferroic orders coexist are called multiferroic materials. One case of these materials are the magnetoelectric (ME) materials, these materials are ferroelectric and ferromagnetic and at the same time they posses a coupling between these two ferroic orders. Recently these materials have attracted an increasing interest due to the discovery of new materials that make possible several applications (For an historical review see section 1.3). The direct magnetoelectric coupling is the appearance of an electrical polarization $P$ when a magnetic field $H$ is applied, this can be expressed by the equation:

$$
\Delta P=\beta \Delta H o r \Delta E=\alpha \Delta H
$$

Where $\Delta E$ is the change in the electric field, $\alpha$ is the ME voltage coefficient and $\beta$ is the ME charge coefficient. This means that the electric polarization can be changed with an external magnetic field or a voltage can be produced with a magnetic field if the material possesses ME coupling. The ME materials not only posses direct ME effect, they also posses the so called converse ME effect. The converse ME effect is the appearance of a magnetization (magnetic polarization) $M$ when a electric field is applied. This can be expressed with the following equation, 


$$
\Delta M=\gamma \Delta E
$$

This means that the magnetization of the material can be modified with an external electric field by the converse ME effect that the ME materials posses. In order to obtain these relations between the electric and magnetic fields in a non homogeneous material and using Cartesian coordinate system a more general deduction has to be made [1]. To do this first the expansion of the free energy of a material has to be considered

$$
\begin{aligned}
F(\vec{E}, \vec{H})= & F_{0}-P_{i}^{S} E_{i}-M_{i}^{S} H_{i} \\
& -\frac{1}{2} \epsilon_{0} \epsilon_{i j} E_{i} E_{j}-\frac{1}{2} \mu_{0} \mu_{i j} H_{i} H_{j}-\alpha_{i j} E_{i} H_{j} \\
& -\frac{1}{2} \beta_{i j k} E_{i} H_{j} H_{k}-\frac{1}{2} \gamma_{i j k} H_{i} E_{j} E_{k}-\ldots
\end{aligned}
$$

with $\vec{E}$ and $\vec{H}$ as the electric field and magnetic field, respectively. Differentiations leads to the polarization

$$
\begin{aligned}
P_{i}(\vec{E}, \vec{H})= & -\frac{\partial F}{\partial E_{i}} \\
= & P_{i}^{S}+\epsilon_{0} \epsilon_{i j} E_{j}+\alpha_{i j} H_{j} \\
& +\frac{1}{2} \beta_{i j k} H_{j} H_{k}+\gamma_{i j k} H_{j} E_{k}-\ldots
\end{aligned}
$$

and to the magnetization

$$
\begin{aligned}
M_{i}(\vec{E}, \vec{H})= & -\frac{\partial F}{\partial H_{i}} \\
= & M_{i}^{S}+\mu_{0} \mu_{i j} H_{j}+\alpha_{i j} E_{j} \\
& +\beta_{i j k} E_{j} H_{k}+\frac{1}{2} \gamma_{i j k} H_{j} E_{k}-\ldots
\end{aligned}
$$


where $\vec{P}^{S}$ and $\vec{M}^{S}$ denote the spontaneous polarization and magnetization and $\hat{\epsilon}$ is the electric permittivity and $\hat{\mu}$ is the magnetic permeability. The tensor $\hat{\alpha}$ correspond to induction of polarization by a magnetic field or of magnetization by an electric field which is designated as the linear ME effect. The matrix $\hat{\alpha}$ shows the coupling between the electric and magnetic fields in a more detailed way, e.g. when a magnetic field is applied in the $\mathrm{x}$ direction an electric polarization can be generated in the y direction because of the non diagonal components of $\hat{\alpha}$. Another advantage of the tensor notation is that shows the non homogeneous behavior of the ME materials, e.g. when a single crystal is used, the different orientations shows different ME coupling and as it will be discussed afterwards the laminate composite materials that present a huge anisotropy since they have ME coupling in only one direction. After the $\hat{\alpha}$ matrix there are higher orders of ME coupling that are not linear e.i. $\hat{\beta}$ and $\hat{\gamma}$. In the vast majority of research on the ME effect this higher order coupling is not investigated and the ME effect is considered to be only the linear ME effect, a convention that will be used in this work also.

Since the first observation of the ME effect a lot of research has been made due to the potential applications, e.g., magnetic sensors, energy harvesters, memory devices, etc. The most promising applications will be summarized in section 1.6. In order to make these applications possible a high linear ME coefficient at room temperature is required. Historically several approaches have been made to achieve these properties in single phase materials and composite materials. These different approaches are reviewed in the next section.

\subsection{Historical review}

The first important achievement in the area of ME effect was made by Rontgen in 1888 [2]. He showed that a dielectric material that is moving can be magnetized when an external electric field is applied. This effect is known as the direct ME effect. After this discovery the converse ME effect was observed by Wilson [3]. He measured the electric polarization of 
a dielectric material (in this case ebonite) when it is set rotating in a magnetic filed parallel to the axis of revolution. He found out that the produced polarization is proportional to the applied magnetic field. In 1894 Pierre Courie [4] first proposed that the ME effect was possible in static materials. He proved this by crystal symmetry considerations, i.e. an asymmetric molecule may become magnetically polarized when one places them in an electric field. He was not able to quantify the effect, meaning that he did not know if it was feasible to measure the effect and under what conditions e.g. temperature of the experiment. The term "magnetoelectric" was coined by Debye [5] a few years after the first (unsuccessful) attempts to demonstrate the static ME effect experimentally. The ME effect was experimentally discovered by Rado et al. [6] back in 1961 using single crystal $\mathrm{Cr}_{2} \mathrm{O}_{3}$. They found that the voltage produced was proportional to the applied electric field. Additionally they characterized the ME response as a function of the temperature, this results can be seen in Figure 1.1. A clear disadvantage of this material is that does not present ME response at temperatures higher than $-70^{\circ} \mathrm{C}$. Another drawback of these ME materials is that the ME coupling is really small for technical applications like ME sensors or harvesters, the maximum value, that can be seen in the Figure 1.1, is $4.13 \mathrm{ps} \mathrm{m}^{-1}$ at $263 \mathrm{~K}$ according to $[1,7]$. In order to increase the ME coefficient several works have been made in materials with other compositions. As a result of this work about 80 materials were found that displays ME effect [8]. The three largest coefficients have been observed for $\mathrm{LiCoPO}_{4}$, yttrium iron garnet (YIG) and $\mathrm{TbPO}_{4}$. The ME effect in $\mathrm{LiCoPO}_{4}$ was reported by Rivera [7]. In this case the value of the ME coupling was $30.6 \mathrm{ps} \mathrm{m}^{-1}$ measured at $4.2 \mathrm{~K}$. They found that the Néel temperature was $21.9 \mathrm{~K}$. In the case of YIG a ME effect of $30 \mathrm{ps} \mathrm{m}^{-1} 1$ was reported by Krichevtsov et al. [9]. The value of the ME effect reported by Rado et al. in $\mathrm{TbPO}_{4}$ was $36.7 \mathrm{ps} \mathrm{m}^{-1}$ [10]. This value was obtained at a temperature of $2 \mathrm{~K}$.

After these investigations with single phase materials, the interest in the ME coupling phenomena decreased. This decrease was due to several reasons. With the understanding of the microscopic mechanisms driving ME behavior it became clear that the coupling would 


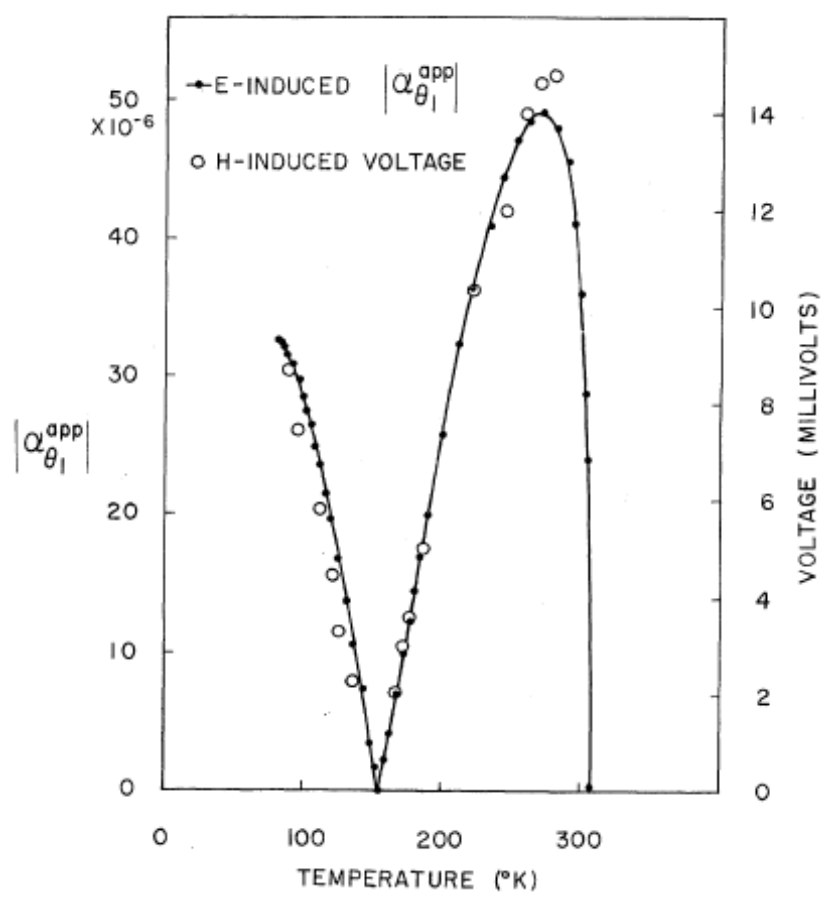

Figure 1.1: Voltage induced at different temperatures on the experiment designed by Rado et al. Reprinted with permission from [6]. Copyright (1961) by the American Physical Society.

hardly exceed about $10 \mathrm{ps} \mathrm{m}^{-1}$, that did not make any application possible. Another reason of this decrease was that the temperature (Curie or Néel temperature) in which these materials show ME coupling is far below room temperature. The discovery of the ME composite materials increased the interest in ME materials again. The drawbacks in the single phase materials were solved and more degrees of freedom in the design of these composites for different performance and applications were available.

\subsection{ME effect in composite materials}

The physical properties of a material that is formed from two or more single-phase compounds are determined by the properties of the constituents as well as by the interaction between them. Two effects can be distinguished [11]. First the sum property, is a weighted sum of the contributions from the constituents phases, the weight being determined by the fractions of these phases. An effect described by $B_{i}=\sigma_{i} A$ with $i=1,2$ denoting the constituents of 
(a) Sum Properties

$$
\left.\begin{array}{l}
\text { Phase } 1: \mathrm{A} \rightarrow \mathrm{B}_{1} \\
\text { Phase } 2: \mathrm{A} \rightarrow \mathrm{B}_{2}
\end{array}\right] \mathrm{A} \rightarrow \mathrm{B}^{*}
$$

\section{(b) Product Properties}

\section{$\left.\begin{array}{l}\text { Phase 1: } \mathrm{A} \rightarrow \mathrm{B} \\ \text { Phase } 2: \mathrm{B} \rightarrow \mathrm{C}\end{array}\right] \mathrm{A} \rightarrow \mathrm{C} \quad$ New Function}

Figure 1.2: Composite properties; (a) sum properties, and (b) product properties. Reprinted from [11], Copyright (2002), with permission from Springer.

a two phase composite, will display the sum effect $B=\sigma A$, with $\sigma=f_{1} \sigma_{1}+f_{2} \sigma_{2}$ and $f_{i}$ as compositional fractions. Density and resistivity are examples of sum properties. Another effect are the product properties; this refer to effects which are present in the composite, but in none of its compounds. The effects $B_{1}=\sigma_{1} A$ and $C_{2}=\sigma_{2} B$ can lead to the product effect $C=\sigma A$ with $\sigma=\phi \sigma_{1} \sigma_{2}$, where $\phi$ is a function of the fractions $f_{i}$ and the connectivity between the constituents. These properties are summarized in Figure 1.2.

Where sum properties denote the average of the effects which are already present in the constituents of the composite, product properties refer to novel effects originated in the interaction between constituents. Composites can therefore be used to generate ME behavior from materials which in themselves do not allow the ME effect. This is conveniently achieved by combining magnetostrictive and piezoelectric materials. A magnetic field applied to the composite will induce strain in the magnetostrictive constituent which is mechanically transferred to the piezoelectric constituent, where it induces an electric polarization. An equation that represents the mechanical coupling between the piezoelectric and magnetostrictive materials is:

$$
\text { MEeffect }=\frac{\text { electrical }}{\text { mechanical }} \times \frac{\text { mechanical }}{\text { magnetic }}
$$




\subsubsection{Discovery of ME effect in composite materials}

The idea of synthesizing a composite medium displaying a ME effect was first formulated by Tellegen in 1948 [12], that is, more than a decade before the first observation of intrinsic ME behavior in single-phase compound. Tellegen suggested a suspension of macroscopic particles that carry both electric and magnetic dipole moments. Actually, van der Boomgaard et al. grew the first artificial ME material by combining ferroelectric piezoelectric $\mathrm{BaTiO}_{3}$ and ferromagnetic $\mathrm{CoFe}_{2} \mathrm{O}_{4}$ in an eutectic composite by unidirectional solidification [13, 14].

The strain caused by a magnetic field in the magnetostrictive materials is not linearly proportional to the field strength but is related to the square of the magnetic field strength. This makes the product property, the magnetoelectric effect in the piezoelectric-magnetostrictive composites, a non-linear effect unlike the single phase materials where the magnetoelectric effect is a linear effect over a wide range of the values of the magnetic or electric field [11]. Also the magnetoelectric effect in these composites shows a hysteresis behavior. This makes the applications of such composites difficult in linear devices. Linearity in such composites is achieved by applying a bias magnetic field across them so that the magnetoelectric effect over a short range around this bias can be approximated as a linear effect. The hysteretic nature of this effect can be used in memory devices, for which there is no necessity of a bias magnetic field.

In composite materials the ME effect is defined for a weak (up to 10 Oe) ac magnetic field applied in the presence of a large (up to $10 \mathrm{kOe}$ ) dc bias field with frequencies of the ac field between $100 \mathrm{~Hz}$ and $1 \mathrm{MHz}$. The voltage produced by the ac field is proportional to the ac field amplitude. This establishes an analogy to the linear ME response of the single-phase compounds and reveals the ME voltage coefficient $d E / d H$, which is typically specified in units of $\mathrm{mV} \mathrm{cm}^{-1} \mathrm{Oe}^{-1}$. This value is calculated with the following formula:

$$
d E / d H=\frac{V_{\text {out }}}{t H_{a c}}
$$


Where $V_{\text {out }}$ is the voltage measured by the amplifier, $t$ the thickness and $H_{a c}$ is the AC magnetic field applied. Depending on the growth conditions the composition used by van der Boomgaard et al. revealed ME voltage coefficients of up to $130 \mathrm{mV} \mathrm{cm}^{-1} \mathrm{Oe}^{-1}$, which

corresponds to $\alpha=720 \mathrm{ps} \mathrm{m}^{-1}$. Thus, even the earliest experiments on composite magnetoelectrics the ME response exceeds the largest values observed on single-phase compounds by more than an order of magnitude [13].

The use of the product properties in ME composites, given by the equation 1.8 , is a mighty addition to the ME coupling phenomena. These composites can be tunned to optimize the performance in each particular application, examples of the new variables in ME composites are; the stoichiometry between the piezoelectric and magnetostrictive phases and the microstructure of the composite.

\subsubsection{ME composites made by ceramic sintering}

After the work done by van der Boomgaard et al. [13] several works have been made using ceramic double sintering process. The unidirectional solidification used by van der Boomgaard et al. requires high temperatures and a critical control over the composition especially when one of the components (oxygen) was gas in order to avoid unexpected third phases in the composite. The ceramic sintering is a much easier and cheaper ME composites fabrication technique compared with unidirectional solidification. Moreover, molar ratios of phases, grain size of each phase, and sintering temperature are easily controllable.

There are three important issues in the fabrication of ME particulate composites that must be addressed in order to achieve a high ME voltage. First, no chemical reaction should occur between the piezoelectric and magnetostrictive materials during the sintering process. The chemical reaction may reduce the piezoelectric or magnetostrictive properties of each phase. Second, the resistivity of magnetostrictive phase should be as high as possible. If the resistivity of magnetostrictive is low, the electric poling becomes very difficult due to leakage current. Also, the leakage current reduces the magnetoelectric properties of the composites. 
When the ferrite particles make long connected chains (percolation), the electric resistivity of composites is reduced significantly, because of the low resistivity of ferrite. Therefore, good dispersion of the ferrite particles in the matrix is highly required in order to sustain sufficient electric resistivity of the composite. Third, mechanical defects such as pores in the interface between two phases should not exist in the composite for good mechanical coupling.

An example of this is the work done by Kothale et al. [15]. In this work they mixed $\mathrm{Cu}_{0.6} \mathrm{Co}_{0.4} \mathrm{Fe}_{2} \mathrm{O}_{4}$ and $\mathrm{Ba}_{0.8} \mathrm{Pd}_{0.2} \mathrm{TiO}_{3}$ powders and sinter them at $1050^{\circ} \mathrm{C}$. In this work, they did not observe any phase interaction between the ferrite and the ferroelectric phase characterized by XRD. They analyzed different stoichiometries; 15,30 and $45 \%$ of magnetostrictive phase. The maximum value of ME coupling was achieved for the lowest amount of magnetostrictive phase. This is due to the fact that when high amounts of magnetostrictive phase are added, the leakage currents drastically decrease the ME coupling.

Another work in ME composites obtained by conventional ceramic sintering is the work made by Zhai et al. [16]. They used $\mathrm{CoFe}_{2} \mathrm{O}_{4}$ for the magnetostrictive phase and PZT for the piezoelectric phase. They characterized the piezoelectric constant $\left(d_{33}\right)$ for different PZT compositions and they found that the presence of $\mathrm{CoFe}_{2} \mathrm{O}_{4}$ decreases the piezoelectric properties, these results can be seen in Figure 1.3. By adding 10 vol\% of $\mathrm{CoFe}_{2} \mathrm{O}_{4}$ to the PZT, the $d_{33}$ decreases three times and when the amount of PZT is equal to the $\mathrm{CoFe}_{2} \mathrm{O}_{4}$ the piezoelectric response is zero. The reason for this is that the resistance of the ferrite is much smaller than that of PZT. This makes the current leak through the composite. When a composite contains too much ferrite, it cannot be poled at high voltage for its low resistance and as a consequence cannot get a good piezoelectric effect.

The Figure 1.4 shows the ME voltage coefficient for various PZT contents. With low PZT content, there is no ME effect due to $d_{33}=0$. As PZT content increases, the $d E / d H$ of the composites increases due to the increase in $d_{33}$. The composites with 80 vol\% PZT shows the higher ME voltage coefficient, with a value of $30.2 \mathrm{mV} \mathrm{cm}{ }^{-1} \mathrm{Oe}^{-1}$. At higher PZT content the magnetostriction of the composites is small which leads to the decrease in $d E / d H$. 


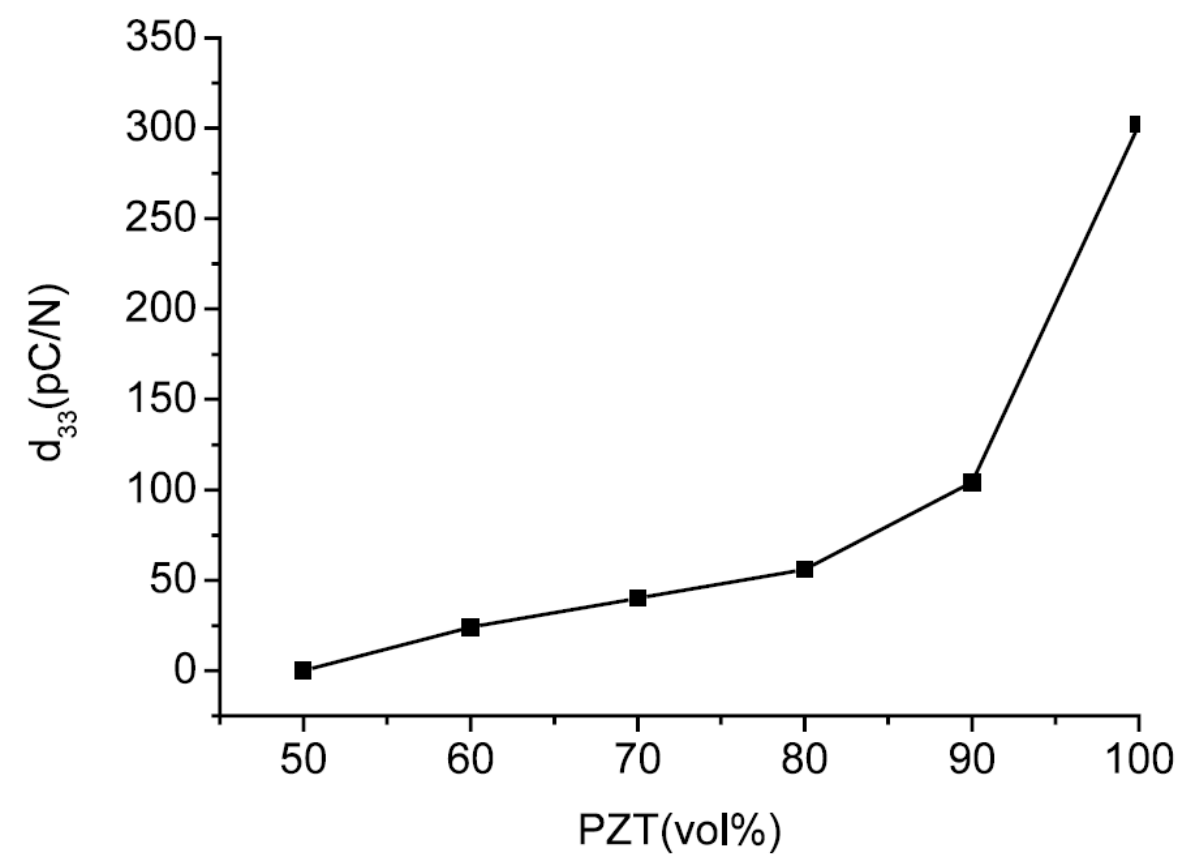

Figure 1.3: Variation in piezoelectric properties for different PZT contents. Reprinted from [16], Copyright (2003), with permission from Elsevier.

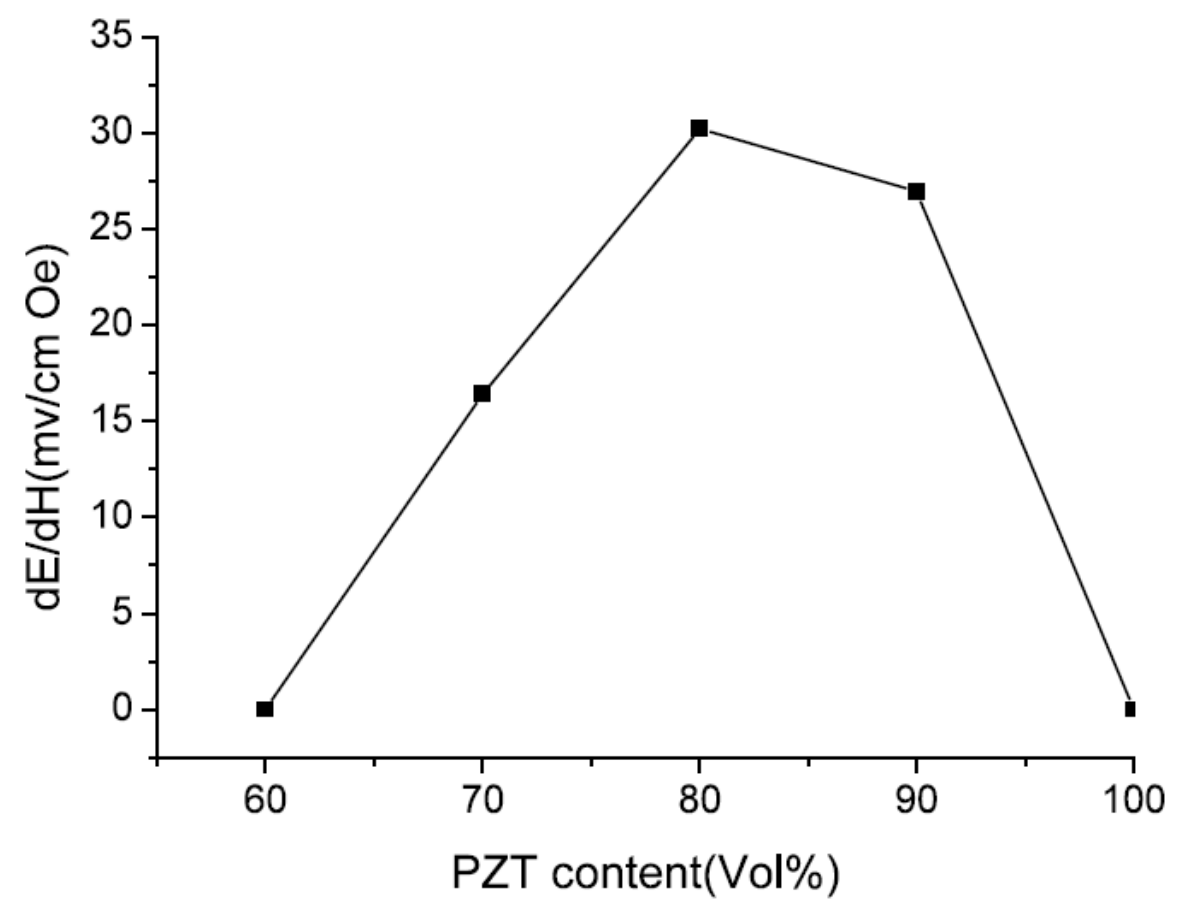

Figure 1.4: Variation of the magnetoelectric properties for different PZT contents. Reprinted from [16], Copyright (2003), with permission from Elsevier. 
Another work in sintered composites is that of Ryu et al. [17]. In this work they used PZT as the piezoelectric phase and $\mathrm{NiCO}_{0.05} \mathrm{Cu}_{0.05} \mathrm{Mn}_{0.1} \mathrm{Fe}_{1.8} \mathrm{O}_{4}$ as a magnetostrictive phase. They used this Ni-ferrite because of its high magnetostrictive coupling and high resistivity They used different compositions of the Ni-ferrite and different sintering temperatures, from 1100 to $1300{ }^{\circ} \mathrm{C}$ to investigate the sintering behaviors, microstructures and piezoelectric and magnetoelectric properties of these particulate composites. The particle size of the Ni-ferrite phase obtained by solid state sintering was in the order of $3 \mu \mathrm{m}$. After mixing the powders and sintering, they did not find any additional phase formation but the peak intensity of the ferrite phase decreased when sintering at $1300^{\circ} \mathrm{C}$. This can be due to the diffusion of the Fe ions into the piezoelectric phase. This detrimental effect can be seen in the piezoelectic and in the magnetoelectric properties. When the temperature was increased from 1150 to $1250{ }^{\circ} \mathrm{C}$ the ME effect increased due to the better connectivity between the ferrite and the PZT grains. With regards of ferrite composition they found that when the concentration is increased the agglomerated clusters size increased. This leads to a decrease in the piezoelectric properties and increase of the conductivity. At the same time, with higher amounts of ferrite phase the magnetostriction of the composite increases. This gives a compromise to obtain the maximum ME coupling. As a result of this study they obtained an optimum ME coefficient of $115 \mathrm{mV} / \mathrm{cm}$ Oe for a $20 \%$ ferrite particulate composite sintered at $1250{ }^{\circ} \mathrm{C}$.

In order to improve the connectivity of the particulate composite, Laletin et al. [18] analyzed the influence in the particle surface area (particle size) in the ME effect of the composite. As a piezoelectric phase they used TsTBS-3 barium lead zirconate titanate and as a magnetostrictive phase they used $\mathrm{NiFe}_{1.9} \mathrm{Co}_{0.02} \mathrm{O}_{4}$. They had chosen this Ni-ferrite because of it's high resistivity, $9 \times 10^{9} \Omega \mathrm{cm}$, that can improve polarization and ensures higher ME performance. In this work they used specific surface areas from 0.8 to $7.6 \mathrm{~m}^{2} / \mathrm{g}$ and sintering temperatures from 1130 to $1210^{\circ} \mathrm{C}$. The effect of the surface area change and temperature can be seen in Figure 1.5; the ME effect increases when the surface area increases and when the sintering temperature increases. There is a $60 \%$ increment of the ME coupling 


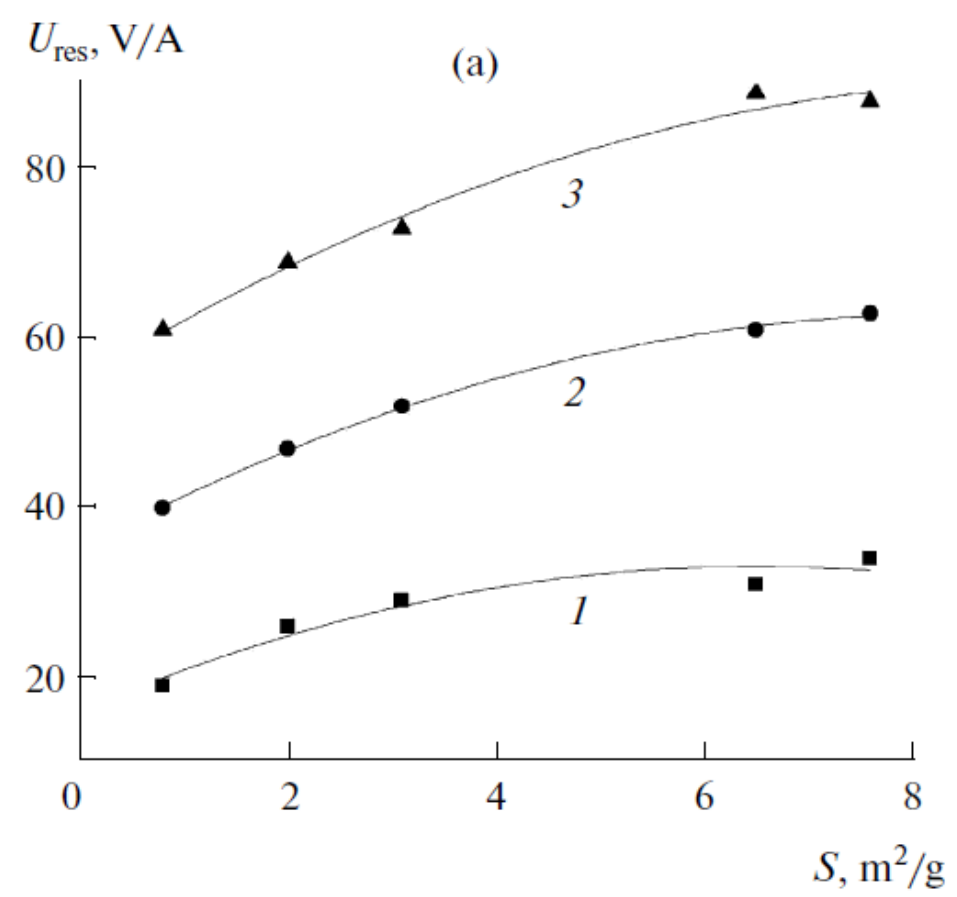

Figure 1.5: ME voltage coefficient for powders obtained at sintering temperatures of (1) 1130, (2) 1170, and (3) $1210^{\circ} \mathrm{C}$. Reprinted from [18], Copyright (2012), with permission from Springer.

with the increment in the surface area. This is due to the formation of an homogeneous composite material given by the better dispersion, improvement of the dielectric properties, increase of the contact area between phases and increase of the density of the material. The maximum ME effect coupling that they obtained was $107.9 \mathrm{mV} / \mathrm{A}$, that is $85.9 \mathrm{mV} / \mathrm{Oe} \mathrm{cm}$, for the samples sintered at $1210^{\circ} \mathrm{C}$ with a powder surface area of $6.5 \mathrm{~m}^{2} / \mathrm{g}$.

As it was demonstrated before, smaller particle sizes improve the ME performance in particulate composites but this effect is valid for grain sizes bigger than $1 \mu \mathrm{m}$. This was demonstrated by Adnan Islam et al. [19]. They studied the effect of the particle size, in the $\mathrm{nm}$ region, in PZT - 20\% CFO particulate composite. They found that the ME coupling decreases when the grain size is smaller than one micrometer and the bigger drop is noticed when the grain size is smaller than $200 \mathrm{~nm}$. This is due to the fact that the domain wall motion is restricted with the grain boundaries in piezoelectric materials. For larger grains, where the size of the domain is smaller than the size of the grains, the movement of the 


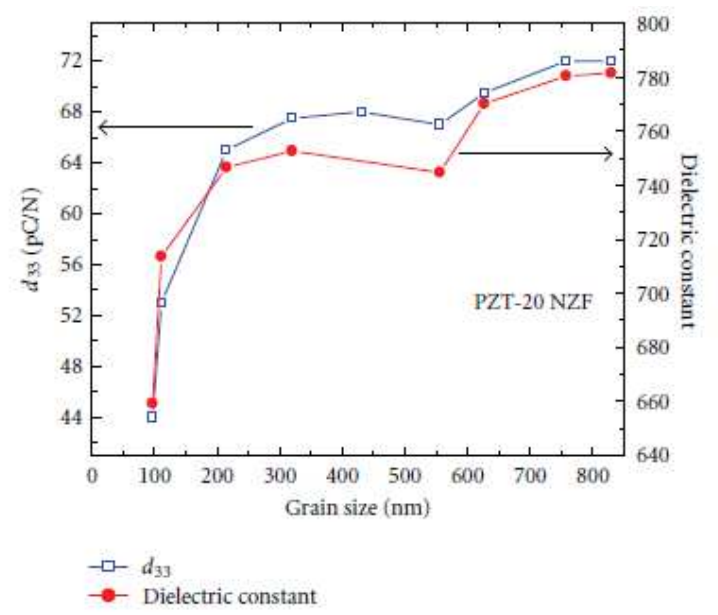

(a)

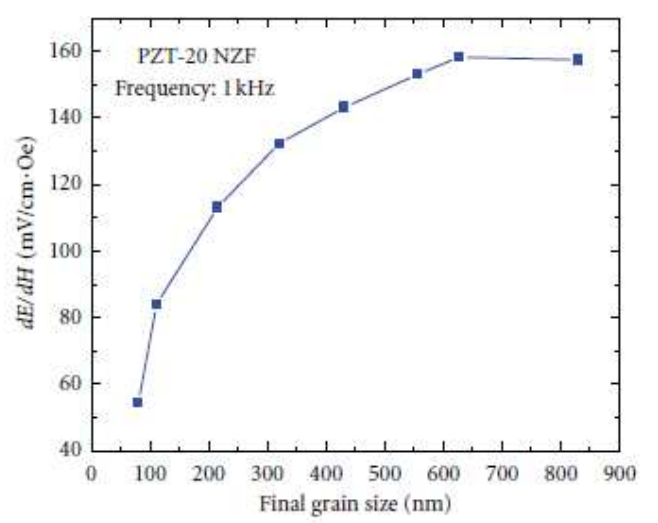

(b)

Figure 1.6: Effect of ferroelectric grain size on (a) the piezoelectric and dielectric and (b) ME coupling of PZT - 20\% NZF composite. Reprinted from [19].

domain walls is easier. On the other hand, in small grains, the movement of domain walls is restricted by the grain boundary. As a result during poling, the domain switching will be difficult which reduces the piezoelectric properties [20]. This will be explained in more detail in section 1.4.3. The results obtained by Adnan Islam et al. can be seen in Figure 1.6. The ME coupling increases from 54.4 to $157.5 \mathrm{mV} / \mathrm{cm}$ Oe when the particle size increases from 97 to $830 \mathrm{~nm}$, respectively. For higher particles sizes the influence is not as important as it is in the nm range. This shows the importance of the grain size with the final ME properties.

Another approach to reduce the electric percolation, leakage currents, is the coating of the magnetic phase as it was made by Nan et al. [21]. He used Tb-Dy-Fe alloy (called TerfenolD) as a magnetic phase, PZT for the piezoelectric phase and PVDF polymer binder. In this 


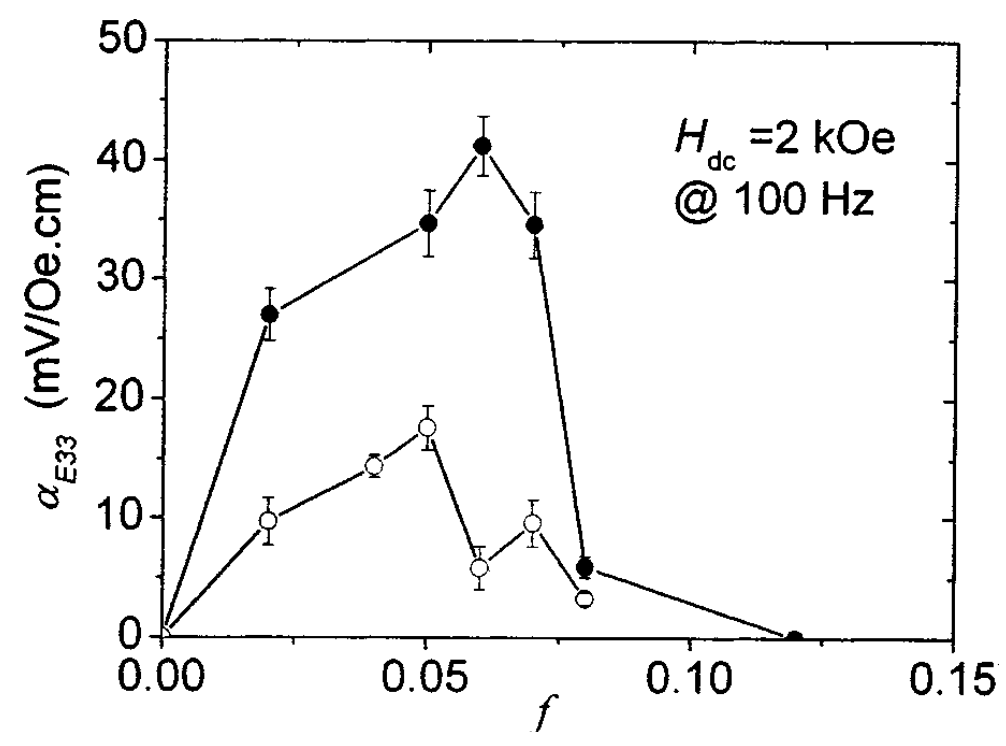

Figure 1.7: $\quad$ ME coupling for different $f$ (Terfenol-D \%) without (solid dots) and with (open dots) surfactant. Reprinted from [21], Copyright (2003), American Institute of Physics.

approach, since the Terfenol-D cannot be heated to high temperatures, a polymer is used to sinter the composite at low temperature $\sim 190^{\circ} \mathrm{C}$ and be able to mechanically transfer the stress generated by the magnetostrictive phase to the piezoelectric phase. A surfactant was added to the Terfenol-D in order to investigate the influence of the particle coating with the leakage currents. The results obtained can be seen in the Figure 1.7. It can be observed that, the addition of the surfactant do not increase the amount of magnetic phase that can be added without having the percolation produced by the leakage currents. Also, the addition of the surfactant decreases the ME coupling; this is due to the decrement of the mechanical transfer of the deformation generated in the magnetic phase. Using Terfenol-D instead of oxides like $\mathrm{CoFe}_{2} \mathrm{O}_{4}$ decrease the amount of magnetic phase that can be added without having leakage currents to $6 \%$. This is the reason why in this system the maximum ME coupling that can be obtained is $42 \mathrm{mV} / \mathrm{Oe} \mathrm{cm}$. 


\subsubsection{Laminate composites}

In order to improve the particulate composites different sintering temperatures, grain sizes and mole fractions were examined as summarized in Section 1.4.2. However, in spite of all these efforts substantial improvement of the ME response of these particulate composites beyond $\sim 100 \mathrm{mV} /$ Oe cm was not achieved, although theories predicted voltage coefficients one or two orders higher than the observed ones. Several reasons for this discrepancy were identified;

- Atomic diffusion between the magnetostrictive and piezoelectric phase generate atomic impurities and sometimes some non desired phases are generated. This is generated due to the high sintering temperatures used to sinter the composite materials $(\sim 1200$ $\left.{ }^{\circ} \mathrm{C}\right)$.

- Mechanical defects between the magnetoelectric and the piezoelectric phase in order to ensure the mechanical transfer of the stress generated in the magnetic to the piezoelectric material cannot be achieved. These defects can be pores or microscopic cracks between the constituents, in order to decrease the porosity and cracks the sintering temperature has to be increased but this has a negative effect because the atomic diffusion increases when the temperature is increased. A compromise between these two effects has to be made in order to obtain the optimum performance. Another option is to change the solid state sintering technique for other techniques where composite without cracks or porosity is obtained at a lower temperature.

- High leakage currents when the magnetic phase composition is increased over $20 \%$. This is due to the percolation of the conductive magnetic phase. This makes a challenge to electrically pole the piezoelectric phase and in addition the ME effect is drastically decreased due to the high leakage currents. Therefore good dispersion of the conducting phase in the piezoelectric matrix in order to avoid contact between particles and reduce the leakage currents is required to improve the ME coupling. With this objective Nan 
et al. [21] used a coating of the magnetic particles to decrease the contact between them and decrease the conductivity, but as a result of this the ME coupling was decreased.

These obstacles were overcome in 2001 by using laminar instead of particulate composites. This discovery was made by Ryu et al. [22, 23]. In this work they stacked, in a sandwich structure, two Terfenol-D disks with a PZT disk. In order to ensure the conductivity of the junction they used silver epoxy to bound the disks. A picture and schematic of the laminate structure can be seen in Figure 1.8. As a result of this structure the ME coupling was increased to $4680 \mathrm{mV} / \mathrm{Oe} \mathrm{cm}$. This value is 40 times higher compared to the ME coupling obtained in the particulate structures. This is mainly because of two reasons; first the leakage currents in the piezoelectric phase are suppressed since the magnetic and piezoelectric phase are not connected and secondly the magnetic and piezoelectric laminae can be fabricated on their own and then be bounded with conductive epoxy at low temperature which prevents from any kind of interaction between the phases. Different PZT compositions were studied in this work and they found that the ME voltage depends linearly with the piezoelectric voltage coefficient $\left(\mathrm{g}_{33}\right)$.

The ME response of the laminated composites is determined by four major aspects which will be discussed; (i) the magnetic, electrical and mechanical coefficients of the constituents, (ii) the respective thickness and number of piezoelectric and magnetostrictive layers, (iii) the type of bonding between constituents, (iv) the orientation of the constituents and the applied fields.

\section{Materials selection}

As it was described before, the materials properties used has a significant effect in the output ME effect. An example of this is the use of different piezoelectric phases in the work made by Ryu et al. [11]. In order to improve the ME coupling he changed the PZT for $\mathrm{Pb}\left(\mathrm{Mg}_{1 / 3} \mathrm{Nb}_{2} / 3\right) \mathrm{O}_{3}-\mathrm{PbTiO}_{3} \mathrm{PMN}-\mathrm{PT}$ single crystal, using the same thicknesses and Terfenol-D laminaes. The piezoelectric voltage coefficient $\left(\mathrm{g}_{33}\right)$ of the PMN-PT and PZT 



Figure 1.8: Magnetoelectric laminate composite using Terfenol-D and PZT disks. (a) schematic structure, and (b) photograph of the device. Reprinted from [23], Copyright (2001), John Wiley and Sons.

are 44.45 and $26.11 \mathrm{mV} \mathrm{m} / \mathrm{N}$, respectively. As a result of the increase of the piezoelectric coefficient, the ME voltage increased from 5.1 to $10.3 \mathrm{~V} / \mathrm{cm}$ Oe for the PZT and PMN-PT, respectively. An increase of $70 \%$ in the piezoelectric constant is transformed into an increase of $101 \%$ in the ME response.

Another example of different piezoelectric phases is the work made by Mori et al. [24]. In this case they used polyvinylidenedifluoride (PVDF) instead of lead based piezoelectric materials. The PVDF has a $\mathrm{g}_{31}$ of $216 \mathrm{~V} \mathrm{~m} / \mathrm{N}$ that is almost 5 times higher compared to the lead piezoelectrics. In this case the Young's modulus must be considered as well because the stress generated in the piezoelectric phase is highly dependent on the Young's modulus.

Particle size is another factor that influences the properties of the piezoelectric and magnetic phases. By controlling the particle size, the electric and magnetic properties can be optimized and as a result an optimum ME coefficient can be achieved. Several works to study the influence in the particle size in the piezoelectric coefficient of the PZT have been 


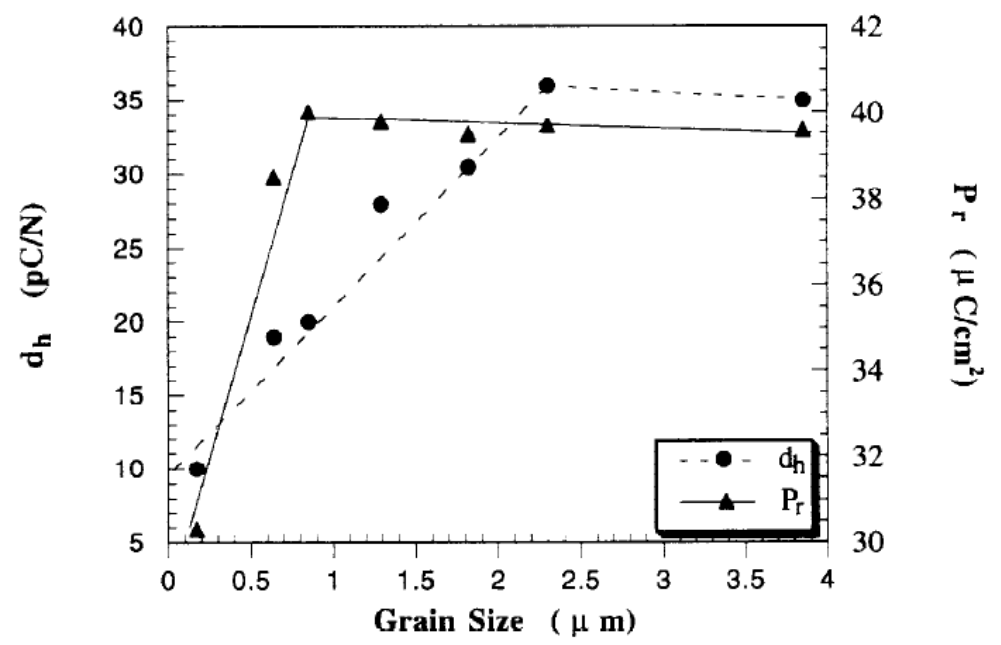

Figure 1.9: Grain-size dependence of remnant polarization $P_{r}$ and hydrostatic coefficient $d_{h}$ for niobium-doped PZT (52/48). Reprinted from [20], Copyright (1998), John Wiley and Sons.

made. An example of this is the work conducted by Randall et al. [20]. In order to obtain different grain sizes they sintered $\mathrm{PbZr}_{0.52} \mathrm{Ti}_{0.48} \mathrm{O}_{4}$ at different temperatures, as it can be expected the grain size increased with the sintering temperature. After this, they measured the remnant polarization and hydrostatic piezoelectric coefficient; these results can be seen in Figure 1.9. The figure shows that the polarization and $d_{h}$ has a critical size around 0.8 and $2.4 \mu \mathrm{m}$, respectively.

In the case of the magnetostrictive phase there is not much work done but a lot of studies have been made in the study of the magnetic properties. It was shown by Uestuener et al. [25] that as particle size decreases (in the $\mu \mathrm{m}$ scale), coercivity increases and for very low value of particle size ferromagnetic to superparamagnetic transition occurs. It has been shown that with increasing particle size from $1 \mathrm{~nm}$ and above, the magnitude of coercive field increases and reaches a maximum before dropping again with further increase in size. The particle size where maximum in coercive field occurs corresponds to the size of the single-domain particle. 


\section{Thicknesses of the layers}

Depending on the materials parameters of each layer, the optimum thickness ratio has to be calculated in order to ensure the optimum ME coupling. When the magnetic phase thickness is too big, compared with the piezoelectric thickness, the composite will produce a large deformation but since the piezoelectric phase is too small it will not produce a large electric field. If we consider the case where the thickness of the piezoelectric phase is bigger than the magnetic phase, there will be good piezoelectric coupling in the composite but the thin magnetic phase will not be able to produce a significant strain in the piezoelectric phase. This is the reason why a compromise of the thickness percentage between the phases is required. This relationship depends on each individual set of magnetic and piezoelectric materials since the magnetostrictive and piezoelectric coupling and the elastic modulus has a big influence on it. A detailed study is made in Chapter 2 and Chapter 3.

\section{Bonding}

Different approaches have been made in order to achieve good mechanical bonding and conductivity between the laminae. Ryu et al. [11] used silver epoxy to bond PMN-PT to Terfenol-D and the laminate was heated to $80{ }^{\circ} \mathrm{C}$ in order to improve the mechanical properties of the epoxy.

A similar technique to bond the piezoelectric and magnetostrictive phases is the one used by Mori et al. [24]. They used $110 \mu \mathrm{m}$ thick PVDF with silver electrodes and $3 \mathrm{~mm}$ thick Terfenol-D. In order to bond these layers they used conductive epoxy and as a result they obtained a ME coupling of $1.43 \mathrm{~V} / \mathrm{cm}$ Oe.

A different approach is the one made by Cai et al. [26]. They fabricated a three phases ME composite with Terfenol D, PZT and PVDF. They made laminae of particulate composites using Terfenol D and PVDF and PZT and PVDF. After this two laminae were fabricated they were stacked and hot molded. Using a hot press ensures good interfacial bounding between composite layers because of the low melting temperature PVDF polymer. The PVDF is used 
as a matrix binder and after the hot pressing at $180{ }^{\circ} \mathrm{C}$ it ensures good mechanical transfer of the stress generated. This structure results in a ME coupling of $90 \mathrm{mV} / \mathrm{cm}$ Oe.

\section{Orientation with the magnetic field}

The magnetic field can be applied in the longitudinal and in the transverse direction giving as a result very different ME couplings. A representative study in the influence of the applied magnetic field is the work made by Srinivasan et al. [27]. They studied the ME coupling for the transverse and the longitudinal mode in $\mathrm{Co}_{1-x} \mathrm{Zr}_{x} \mathrm{Fe}_{2} \mathrm{O}_{4}$-PZT laminate composites. Since the deformations produced in the PZT are small, the voltage generated is proportional to the deformation that the magnetic phase produces. The magnetic phase can only transfer the in-plane deformation because the out of plane direction cannot produce any strain in the laminate structure. Then if we apply a magnetic field in the plane the magnetostriction will be proportional to $\lambda_{11}$ and if the magnetic field is applied perpendicular to the plane the in-plane magnetostriction will be given by $\lambda_{13}$. In the work made by Srinivasan et al. they measured the magnetostriction $\lambda_{11}$ and $\lambda_{13}$ for different applied magnetic fields. The results obtained can be seen in Figure 1.10. It can be clearly seen that the $\lambda_{11}$ is two times higher compared with $\lambda_{13}$. Another important factor that can be seen here is that the saturation magnetization is highly dependent on the composition of the $\mathrm{Co}_{1-x} Z \mathrm{Zr}_{x} \mathrm{Fe}_{2} \mathrm{O}_{4}$, when $\mathrm{x}=0$ the saturation of $\lambda_{11}$ is at $5 \mathrm{kOe}$ but when $\mathrm{x}=0.2$ and 0.4 the saturation magnetization is $2 \mathrm{kOe}$ and $0.6 \mathrm{kOe}$, respectively. The maximum magnetostriction that can be achieved depends on the composition of the CZFO too, being maximum for $\mathrm{x}=0$ and 0.2 .

As it was mentioned before, the ME coupling is directly related with the magnetostriction. But since the ME effect is measured as the change in voltage with the change in $\mathrm{AC}$ magnetic field the ME effect follows the derivative of the magnetostriction with respect to the applied magnetic field. This effect can be seen in the Figure 1.11. Here when the magnetostrictive phase is saturated, there is no voltage generated with the $\mathrm{AC}$ magnetic field and the maximum value of the ME effect is achieved when the slope of the magnetostriction is maximum. 


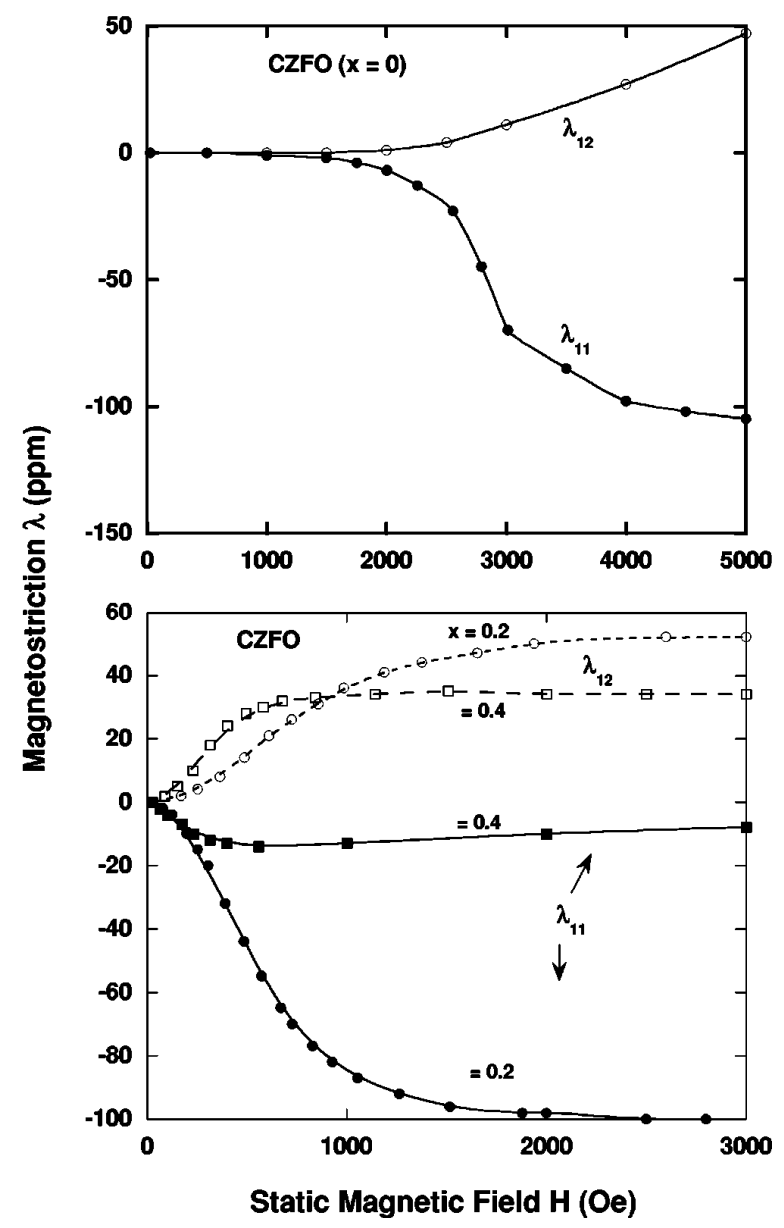

Figure 1.10: Room-temperature in-plane parallel $\left(\lambda_{11}\right)$ and perpendicular $\left(\lambda_{13}\right)$ magnetostriction versus magnetic field for CZFO bulk samples $(\mathrm{x}=0,0.2,0.4)$ made from thick films. Reprinted with permission from [27]. Copyright (2003) by the American Physical Society.

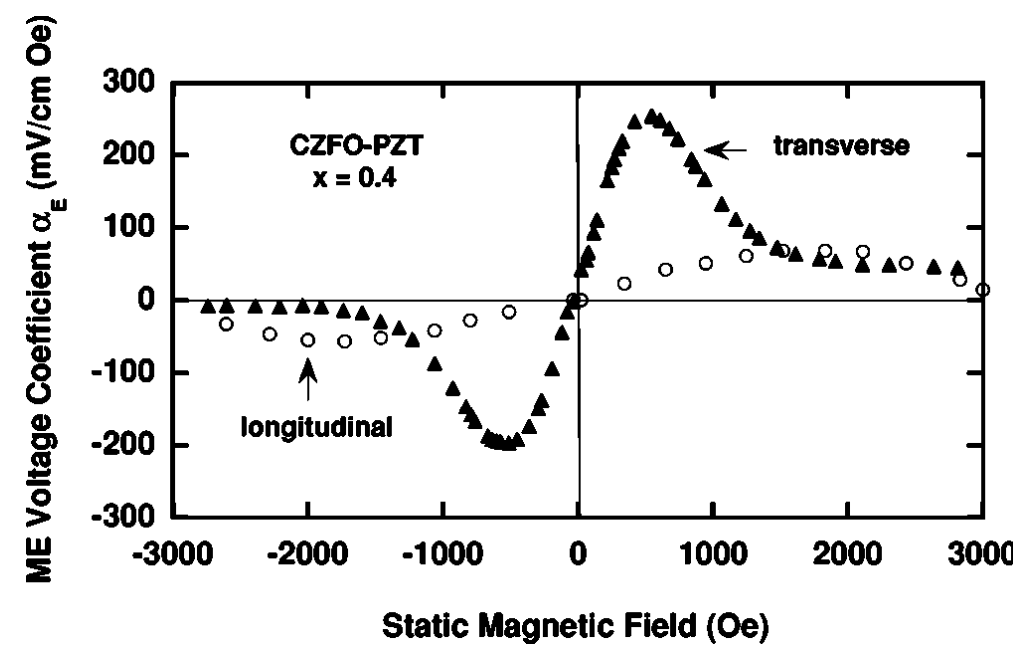

Figure 1.11: ME coupling for CZFO-PZT composite in longitudinal and transverse mode. Reprinted with permission from [27]. Copyright (2003) by the American Physical Society. 
The effect of the magnetic field and the polarization orientation in the ME properties of the composite is further discussed in Chapter 2 and Chapter 3.

\subsubsection{Resonance frequency}

Up to now the influence of the $\mathrm{AC}$ magnetic field frequency that is used in order to generate

the ME response has not been discussed. The reason for this is that for the frequency used (1 - $10^{4} \mathrm{~Hz}$ ) the ME effect remains constant, independently of the frequency. In 2003 Bichurin et al. investigated the influence of higher $\mathrm{AC}$ frequencies in the ME response [28]. They used a laminate composite made of a multilayered composite, 22 layers, of $\mathrm{NiFe}_{2} \mathrm{O}_{4} \mathrm{NFO}$ and PZT with thicknesses of 13 and $26 \mu \mathrm{m}$, respectively. The response of this laminate composite with the frequency can be seen in Figure 1.12. Here it can be seen that this material possesses an electromechanical resonance at $350 \mathrm{kHz}$. If we compare the $\mathrm{ME}$ coupling at $100 \mathrm{~Hz}$ to $350 \mathrm{kHz}$ the values obtained are 30 and $1200 \mathrm{mV} / \mathrm{cm}$ Oe. This is an increase of 40 times when the ME composite is excited at its resonance frequency compared with low frequencies. This effect in the electromechanical resonance can be seen in the impedance response of the composite also. When close to the resonance frequency the impedance has a peak, as it can be seen in Figure 1.12.

\subsection{Thin films ME composites}

The investigation of ME nano-structured films has recently been accelerated by the advances in thin-film growth techniques. These techniques have provided routes for novel structures and phases, and have the properties of traditional functional materials modified by strain engineering and inter-phase control. An ever-increasing number of works have been devoted to research of composite ME films in the last seven years. So far, a number of ME films of ferroelectric (e.g., BTO, $\mathrm{PbTiO}_{3}$ (PTO), PZT and BFO) and magnetic (e.g., CFO, NFO, $\mathrm{Fe}_{3} \mathrm{O}_{4}, \mathrm{La}_{1-x} \mathrm{Sr}_{x} \mathrm{MnO}_{3}$ (LSMO) and metals) with different nano-structures (e.g., 0-3 type 


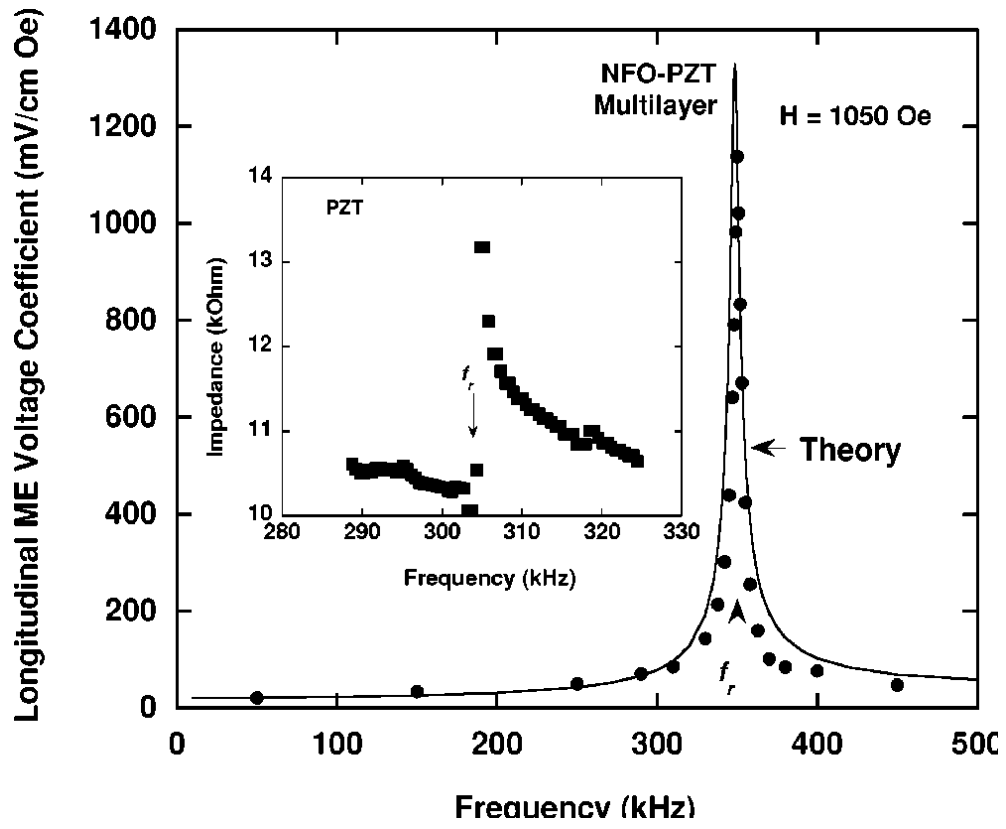

Figure 1.12: Variations of longitudinal ME coupling with different frequencies. The inset, impedance vs. frequency for a 10-mm disk of PZT. Reprinted with permission from [28]. Copyright (2003) by the American Physical Society.

particulate films, 2-2 type layered structures, and 1-3 type vertical structure) have been prepared via physical deposition techniques (e.g., PLD, molecular beam epitaxy (MBE) and sputtering) and chemical processing (e.g., spin coating and metal-organic chemical vapor deposition (MOCVD)).

Compared with bulk ME composites, composite thin films have unique superiorities. Different phases could be combined at atomic-level, and by precise control of the lattice matching, epitaxial or superlattice composite films can be designed, facilitating the understanding of ME coupling at atomic scale. The thin film technology allows to integrate ME composites into a circuit in a confined space. To achieve this, preparation of ME composite thin films with high quality is highly desired, which can be achieved by utilizing a wide variety of grown techniques like PLD, MBE, sputtering, spin coating, MOCVD, and more. 


\subsection{Applications}

\subsubsection{Magnetic sensors}

The working principle of magnetic sensing in the ME composites is simple and direct. When probing a magnetic field, the magnetic phase in the ME composite strains, producing a proportional charge in the piezoelectric phase. Highly sensitive magnetic field sensors can be obtained using the ME composites with high ME coefficients. The ME composites can be used as a magnetic probe for detecting ac or dc fields.

Dong et al. developed a trilayer laminate made of Terfenol-D and PMN-PT that shows induced voltages for magnetic fields down to $10^{-12} T$ [29, 30]. The induced voltage showed a linear response with the applied magnetic fields. Different frequencies of the applied magnetic field were used, a significant improvement was observed when the resonance frequency was used. Magnetic fields at ultra low frequencies were detected using a multilayered ME composite with high ME charge coupling and large capacitance [31]. This sensor reported a sensitivity of $10^{-7} \mathrm{~T}$ at frequencies as low as $10 \mathrm{mHz}$.

In the case of detection of dc magnetic fields, a small constant drive AC field is required [30]. This configuration showed a sensitivity limit of about $10^{-7} T$, using a constant amplitude low frequency drive, which can be enhanced to $10^{-8} T$ under resonant drive.

\subsubsection{Magnetoelectric harvesters}

The ME composites can also be used as transducers. These specific transducers, that transform magnetic fields and mechanical stresses to electrical energy through the ME coupling, are called magnetoelectric harvesters. The magnetic and mechanical energy is present in most environments; this makes them a convenient way to power wireless sensors, mobile electronics and other devices in remote locations [32]. The environment power sources for the harvesters can be, vibrations, human motion, acoustic noise, and also electromagnetic energy coming from the environment such as radio and television broadcasting. 
Recently the wireless technology allow us to place sensors in multiple locations without the necessity of wires. This allows to monitor factories, the human body and the ocean in more remote locations [33]. Due to the fact that the wireless sensors do not rely on wires to transmit the data, they have to be powered with batteries or any other wireless power system. In the case of wireless sensors powered by batteries, the useful life of the sensor is determined by the batteries volume. In many of the sensor applications, the replacement of the batteries is extremely challenging and in some cases impossible. This opens the necessity of a new system to power these devices and recharge them in order to avoid the replacement or the volume increment of the batteries.

The first vibration harvesters were made with piezoelectric materials. These devices transform mechanical vibrations to electrical energy [34, 35]. Here, the amount of energy generated depends fundamentally on the quantity and form, of the mechanical vibrations available, and the efficiency of the particular harvester. The harvester can be made with different geometries in order to achieve the maximum coupling between the environmental vibrations and the transducer. This requires a tuning of the harvester with the particular vibrations of the environment; this means that the resonance frequency of the device should match the frequency of the energy source in order to achieve maximum coupling. The resonance frequency is given by the harvester geometry. Usually, this geometry consists in a frame that is attached to a mass, when it is exposed to vibrations the frame and the inertial mass has a relative displacement. This relative displacement can be transformed to energy with the appropriate transducer, for example a piezoelectric material. The piezoelectric materials transform the relative displacement to polarization that can be stored with the appropriate electronics. The mechanical system can be improved with more complex geometries including hydraulic systems or changing linear displacement to rotary displacement [34].

An example of these devices is the piezoelectric vibrational energy harvester developed by Andosca et al. [36]. They made a micro scale cantilever with a dead mass in the end. This structure was constructed using a micro-fabrication technique over a $\mathrm{Si}$ wafer. In this 


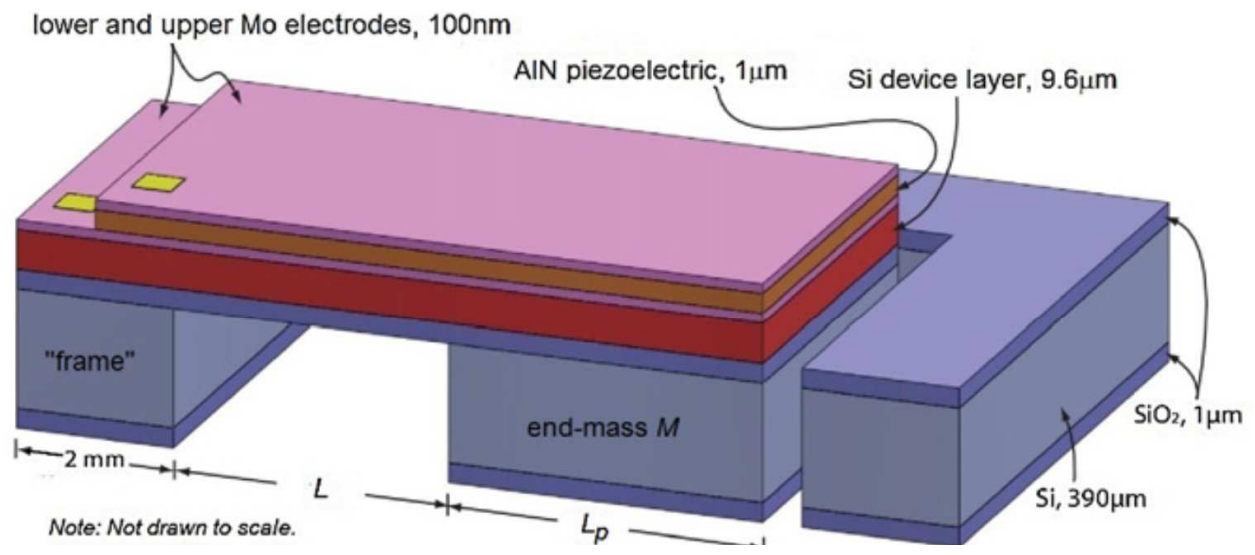

Figure 1.13: Illustration of the piezoelectric cantilever harvester developed by Andosca et al. Reprinted from [36], Copyright (2012), with permission from Elsevier.

harvester they used $A l N$ (piezoelectric material) as active material. The main advantages of $A l N$ over PZT is that it does not have lead, which may cause hazard to the environment and is easier to deposit using a sputtering station due to the fact that there are less elements involved and no oxidation is required. An illustration of this cantilever can be seen in Figure 1.13. The cantilever is composed by the structural layers, like the $\mathrm{Si}$ and $\mathrm{SiO}_{2}$, and the active material $(A l N)$ and electrodes $(M o)$. The end-mass is used to tune the resonance frequency and increase the strain generated in the piezoelectric material. This device was tested at its resonance frequency, of $58 \mathrm{~Hz}$, with an external acceleration of $0.7 \mathrm{~g}\left(\mathrm{~g}=9.81 \mathrm{~m} / \mathrm{s}^{2}\right)$ and as a result generated a power of $63 \mu \mathrm{W}$. If we consider the volume of the device, $1 \mathrm{~cm}^{3}$, it gives a power density of $90 \mu \mathrm{W} / \mathrm{cm}^{3} \mathrm{~g}$. This harvester was successfully used to power a wireless sensor node using a Harvester 3 with the THINERGY IPS-EVALEH-01 Energy Harvesting Evaluation Kit provided by Infinite Power Solutions (IPS; www.infinitepowersolutions.com). This demonstration was made in the laboratory conditions but in many applications this harvester could be used due to the fact that the acceleration and frequency used are similar to those found in industries using $60 \mathrm{~Hz} \mathrm{AC}$ electricity. For other applications the resonance frequency should be tuned to match the excitation frequency.

In the case of ME harvesters several works have been made since $2003[32,33,37,38$, 39, 40]. Dong et al. [32, 37] developed a ME harvester that can synchronously harvester 


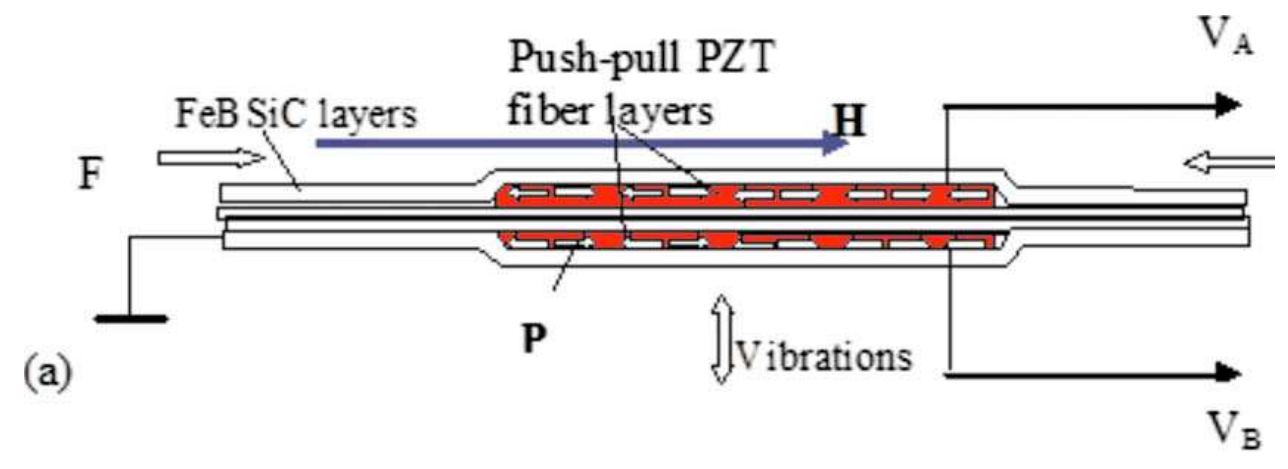

(b)

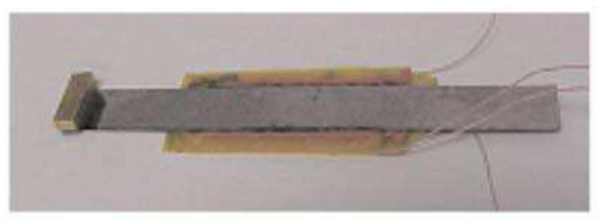

Figure 1.14: (a)Schematic of the ME laminate configuration and (b) photo of the ME laminate proposed by Dong et al. Reprinted with permission from [32]. Copyright [2008], American Institute of Physics.

mechanical and magnetic energy. This was achieved through a ME laminate composite made of piezoelectric PZT fibers stacked between two magnetostrictive FeBSiC alloy laminae. The ME laminate configuration can be seen in Figure 1.14. This ME composite is able to harvest magnetic fields using the longitudinal vibrating mode and harvest mechanical vibration using the bending mode. Regarding the magnetic harvesting, they applied an AC magnetic field of 1 Oe. The AC frequency was set at the mechanical resonance $(\sim 21 \mathrm{kHz})$ of the laminate in order to achieve maximum power. In order to calculate the generated power, they applied an external load of $50 \mathrm{k} \Omega$, giving a resultant power output of $420 \mu \mathrm{W} /$ Oe. Dividing by the volume of the harvester, an output power density of $2.1 \mathrm{~mW} / \mathrm{Oe} \mathrm{cm}^{3}$ was obtained from this work. Since there are not many magnetic sources at $\sim 21 \mathrm{kHz}$, they customized the geometry in order to change the resonance frequency to 20-40 Hz. With this geometry they achieved a power density of $0.4 \mathrm{~mW} / \mathrm{Oe} \mathrm{cm}^{3}$. In this work they harvested mechanical vibrations while magnetic fields were harvested also. 


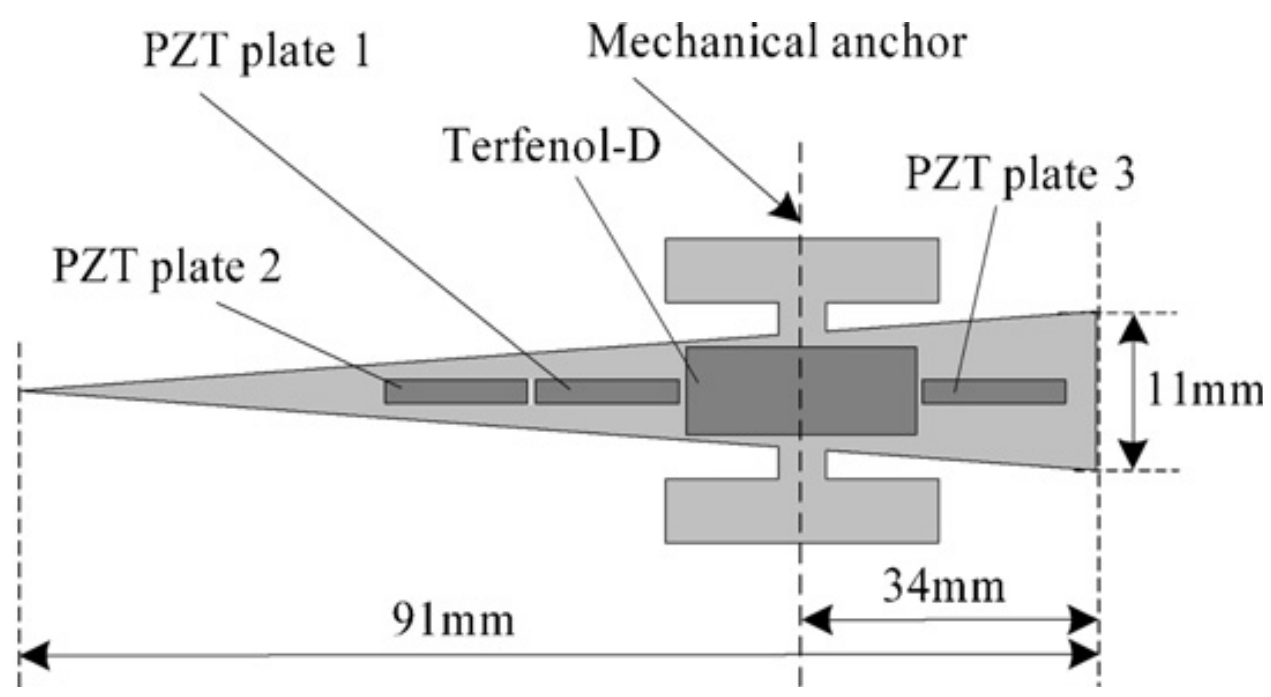

Figure 1.15: Schematic of the ME laminate configuration proposed by Li et al. Reprinted from [38], Copyright (2010), with permission from Elsevier.

A similar work was completed by Li et al. [38]. They made a ME energy harvester with PZT plates and Terfenol-D plate on an ultrasonic horn substrate. The ME composite is placed over an ultrasonic horn in order to increase the oscillation displacement generated by the magnetostrictive plate [41]. This composite ultrasonic horn can be seen in Figure 1.15. With this configuration, they obtained a ME voltage coefficient of $2 \mathrm{~V} / \mathrm{Oe}$ and a power coefficient of $20 \mu \mathrm{W} /$ Oe at resonance. Dividing by the volume of the device $\left(1.5 \mathrm{~cm}^{3}\right)$, it gives a ME power density of $13.3 \mu \mathrm{W} / \mathrm{Oe} \mathrm{cm}^{3}$. This is six times higher than the ME power output obtained by Dong et al. [32], showing the advantages of the ultrasonic horn geometry. With this ME composite they designed an electronic setup that is able to accumulate the weak power produced by the ME harvester and provide a high power output in a short cycle. This is achieved by a switching circuit. When the circuit is charging the storage capacitors are in parallel, and when the circuit is discharged, the capacitors are switched to serial giving as a result a higher voltage/power for a short period when discharging. This device was used to power a temperature and humidity wireless sensor node. This sensor was powered wirelessly by an 1 Oe ac magnetic field generated in the main node. The sensor was successfully operated at a distance of 60 meter without a loss of data and an operation cycle of $620 \mathrm{~ms}$. 


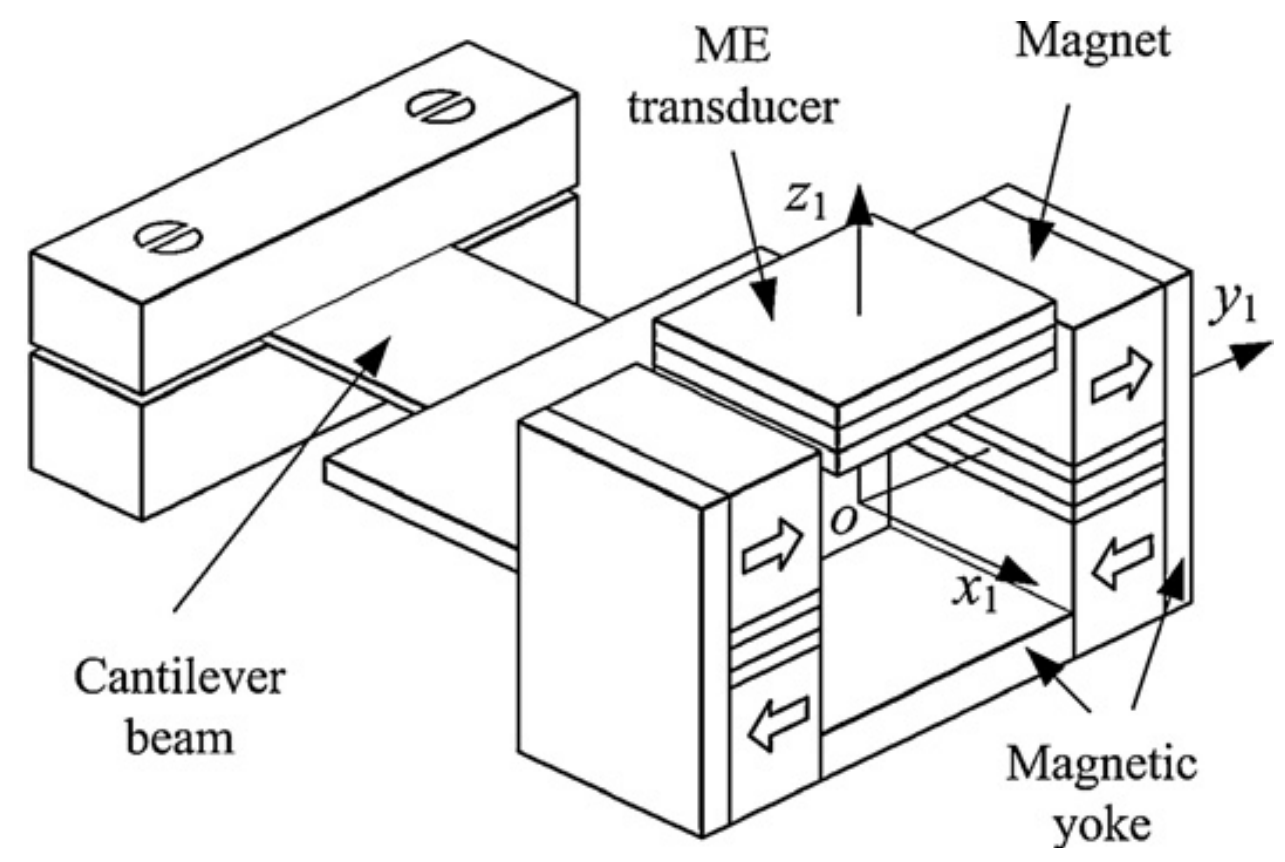

Figure 1.16: Schematic diagram of the vibration energy harvester proposed by Dai et al. Reprinted from [43], Copyright (2009), with permission from Elsevier.

A different approach is to use ME composites to harvest mechanical vibrations [39, 40, 42]. In order to achieve this, the devices generate an $\mathrm{AC}$ magnetic field from the mechanical vibrations, for example a vibrating permanent magnet, and this AC magnetic field is harvested by the ME composite. At the same time the mechanical vibrations are transmitted to the ME composite generating more power. This configuration gives, as a result, a more efficient way to transform mechanical vibrations to useful electric power. An example of these devices is the one proposed by Dai et al. [43]. In this work they generated an AC magnetic field with a setup of permanent magnets vibrating due to the mechanical vibrations. This AC magnetic field was transformed to electrical power with a ME composite. A schematic diagram of the proposed vibration energy harvester can be seen in Figure 1.16. In this case, the ME composite was made of two layers of Terfenol-D surrounding a PZT layer. The power density output that they obtained was of $0.47 \mathrm{~mW} / \mathrm{cm}^{3} \mathrm{~g}$. This is 5 times higher than the electrical power obtained with piezoelectric harvesters.

Another approach was developed by Huang et al. [42]. They developed a ME vibrations 

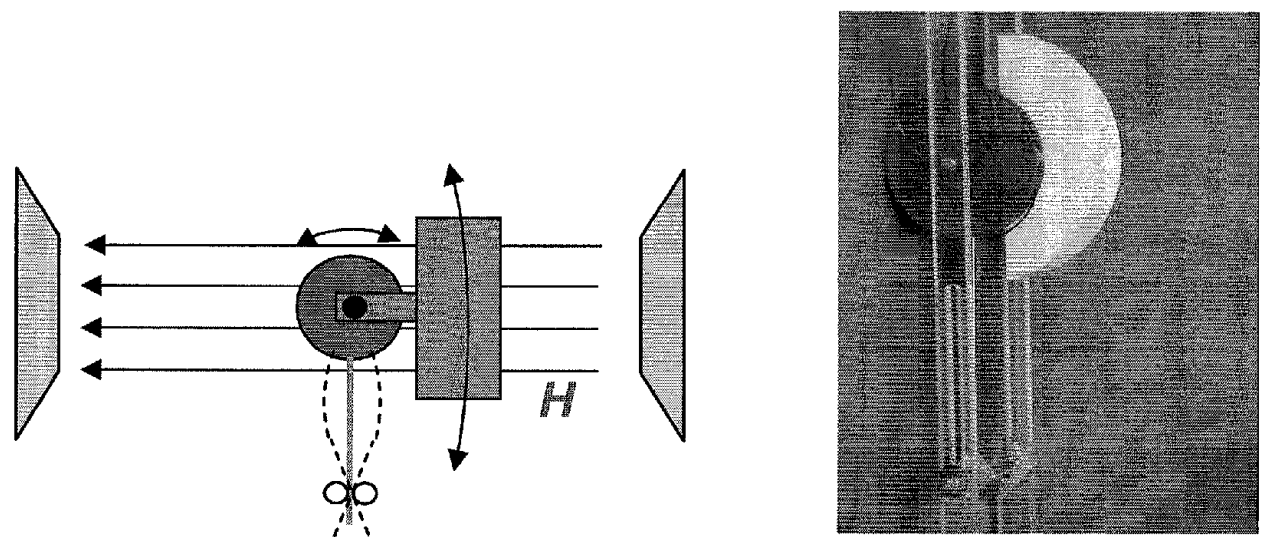

Figure 1.17: Schematic and photograph of the rotational vibration energy harvester developed by Huang et al. Reprinted from [42], with permission from SPIE.

harvester made out of a ME composite composed of two Terfenol-D magnetostrictive layers and an internal PZT layer with a total thickness of $5 \mathrm{~mm}$. They developed a harvester that transforms the external vibration energy into a rotation of the ME composite. This ME composite is set in a constant magnetic field. Due to the relative motion of the ME composite, the magnetic energy is transformed to an AC magnetic field. As a result of the mechanical vibrations, the ME composite is able to produce a polarization that can be externally stored. The schematic of this circular harvester system can be seen in Figure 1.17. This setup is able to harvest up to $10 \mathrm{~mW}$ of electrical power when it is exposed to an acceleration of $0.5 \mathrm{~g}$ at $30 \mathrm{~Hz}$. Considering the volume of only the ME composite $\left(1 \mathrm{~cm}^{3}\right.$ in this example) the power density of this harvester was found to be $20 \mathrm{~mW} / \mathrm{cm}^{3} \mathrm{~g}$. Note that in this calculation only the volume of the ME composite is considered and not the magnets and the beams needed for the ME harvester.

Moss et al. developed another setup that transforms mechanical vibrations using a ME composite [40]. The device consists of a permanent magnet with a ME composite attached to the top. In order to generate an electric polarization through the ME composite, a chrome ball is placed on the top of the composite; when the ball vibrates as a reaction of the external vibrations, it generates an $\mathrm{AC}$ magnetic field over the $\mathrm{ME}$ composite that is transformed to electrical power. A schematic diagram of the energy harvester can be seen in Figure 1.18. 


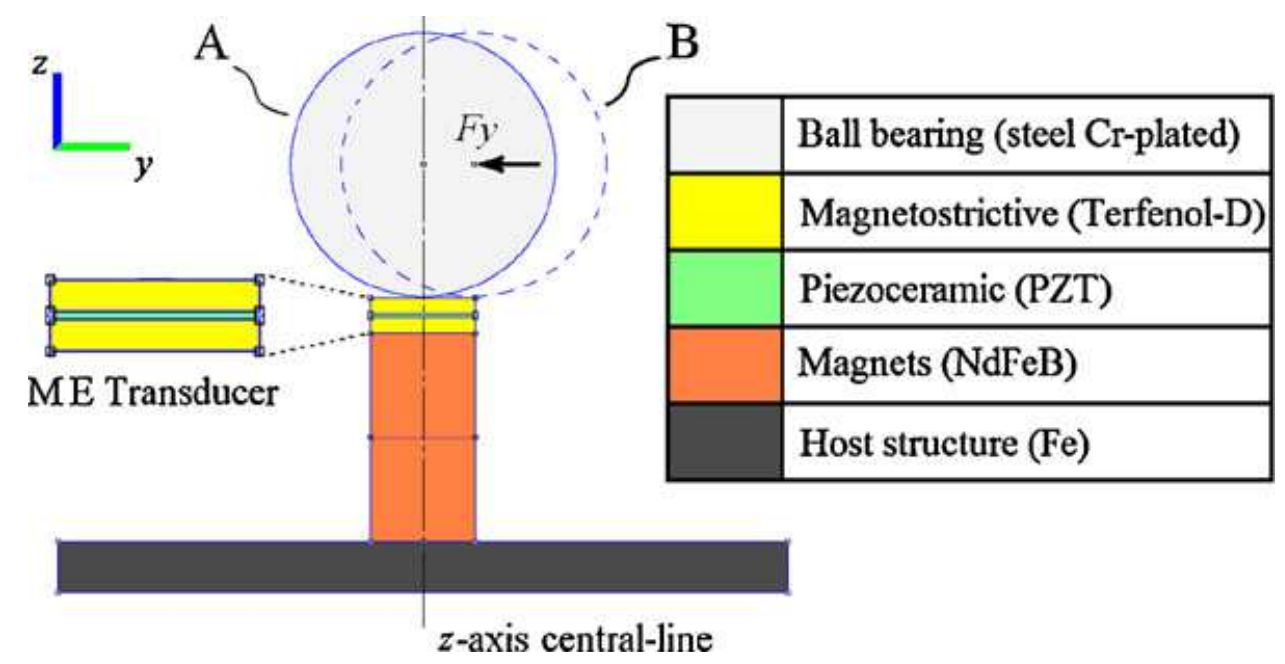

Figure 1.18: Schematic diagram on the energy harvester developed by Moss et al. Reprinted from [40], Copyright (2012), with permission from Elsevier.

This setup produces a power of $121 \mu \mathrm{W}$ when a $61 \mathrm{mg}$ acceleration is applied. Considering the volume of the device $\left(31 \mathrm{~cm}^{3}\right)$, the power density of the device is $0.064 \mathrm{~mW} / \mathrm{cm}^{3} \mathrm{~g}$. This set up produces 7 times less energy than the proposed by Dai et al., but it shows that different configurations could improve the performance of the device [43].

A problem with all of the above devices is that the frequency tolerance of the vibration source is too small; this means that the frequency of the mechanical vibrations has to be close to the resonance frequency in order to produce a high power output. This is a big drawback, due to the fact that in nature waste vibrations are present in a broad and non-constant frequency spectrum. In order to solve this problem, several solutions have been proposed in literature, such as active/passive tuning techniques [44] and widening of the bandwidth [39]. In active/passive tuning techniques, the parameters of the generator such as the mass or the stiffness are altered so that the resonance frequency is tuned to match the environmental frequency. In the active tuning technique, this adjustment is done continuously, this means that the tuning mechanism has to continuously supply power to achieve the resonance frequency change. Whereas in the case of passive tuning technique, the tuning actuators supply power initially to tune the frequency, and then turn off after the adjustment, maintaining the new 


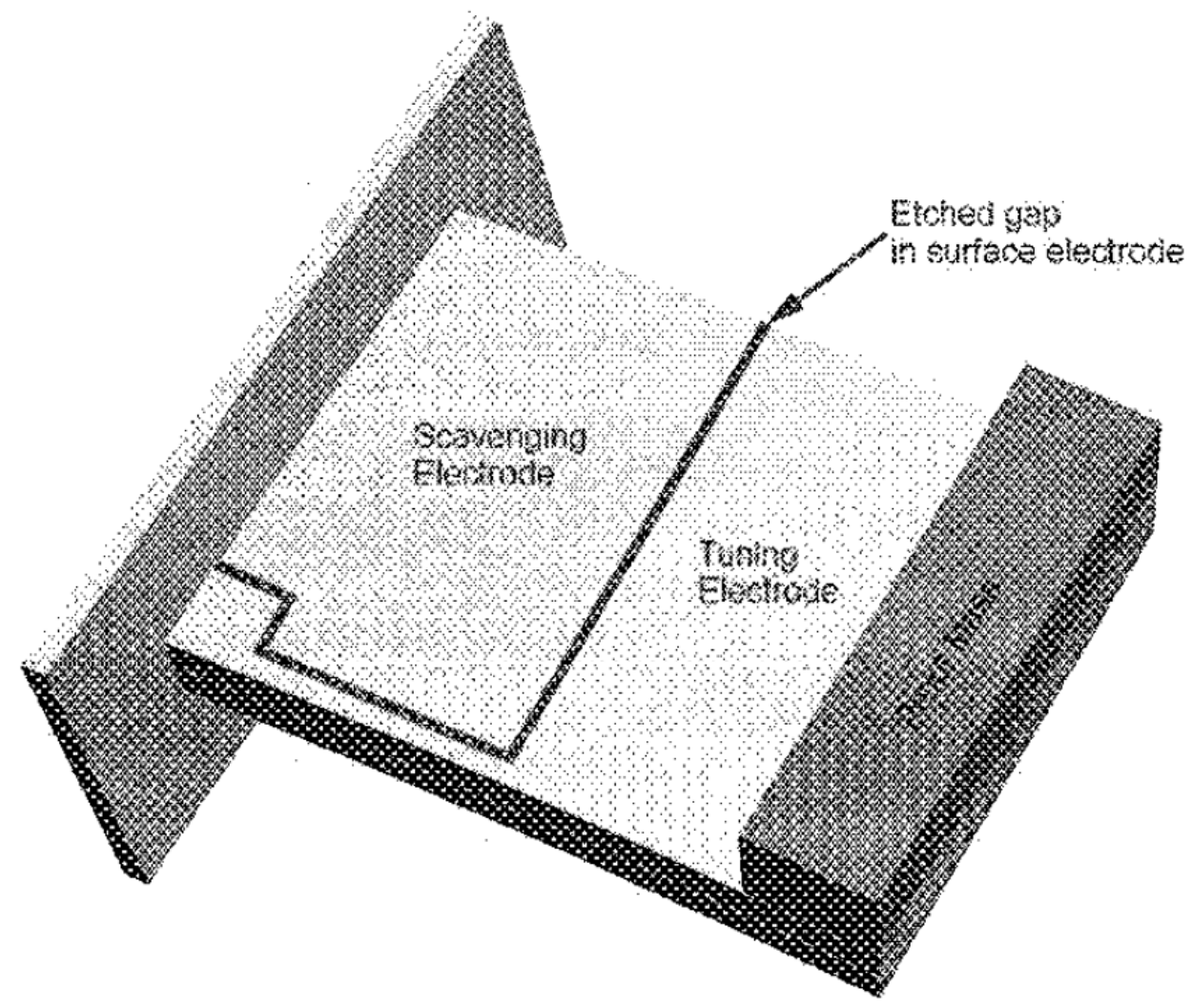

Figure 1.19: Schematic of active piezoelectric vibration harvester developed by Roundy et al. Reprinted from [44], with permission from SPIE.

resonance frequency.

Roundy et al. [44] developed an active tuning device based on a cantilever beam made out of PZT with two different electrodes. One of the electrodes is used to collect the generated power produced by the vibrations and the other half is used for frequency tuning. When a voltage is applied though the tuning electrode the apparent stiffness of the cantilever change produces a change in the resonance frequency. A schematic of this cantilever beam can be seen in Figure 1.19. As it was shown in this work, mathematically and experimentally, active tuning techniques are not feasible because the tuning actuators require more power than the power that the device can generate. On the other hand, passive tuning techniques also require actuators and sensors, which increase the complexity and the cost of the device.

Another solution to improve the operational frequency of the harvesters is to widen the 
bandwidth of the generator. Sari et al. [45] developed a generator that instead of using piezoelectric materials they used a electro-magnetic power generator as a harvester. These devices consist in a coil and a permanent magnet. The power is generated when the relative distance between the magnet and the coil is changed, for this the coil is placed over a cantilever. Under vibration of the cantilever, the relative distance between the coil and the magnet changes generating an electric power. In order to harvester a broad spectrum of frequencies, they developed a series of cantilevers with varying lengths in order to obtain different resonance frequencies of each cantilever. The proposed set up can be seen in the Figure 1.20 a. They derived an expression for the power output in order to calculate the optimum cantilever length distribution and correspondent resonance frequency response. In order to derive this expression, they used the Newton's 2nd law and an electrical equivalent circuit. To obtain a broad harvesting frequency the output of different cantilevers is superposed. This response was predicted by the expression obtained by Rundy et al. and it can be seen in Figure 1.20 b. With the results obtained with this analysis, they fabricated an array of 40 cantilevers with different resonance frequencies. This device generates $0.4 \mu \mathrm{W}$ continuously within a frequency band of $800 \mathrm{~Hz}(4.2-5 \mathrm{kHz})$.

Another approach to broaden the bandwidth of the harvester was made by Yang et al. [39]. The system that they developed consists of two cantilever beams, a permanent magnet and a ME transducer. These two cantilever beams have two different resonance frequencies. With this configuration if one of the cantilevers is vibrating the relative distance between the magnets and the ME transducer will change, generating a power outlet. A schematic diagram of this set up can be seen in Figure 1.21. With this setup they were able to harvest a bandwidth of $4.6 \mathrm{~Hz}$, from 27.2 to $31.8 \mathrm{~Hz}$ giving a constant power output of $2.55 \mathrm{~mW} / \mathrm{cm}^{3} \mathrm{~g}$. 
(a)
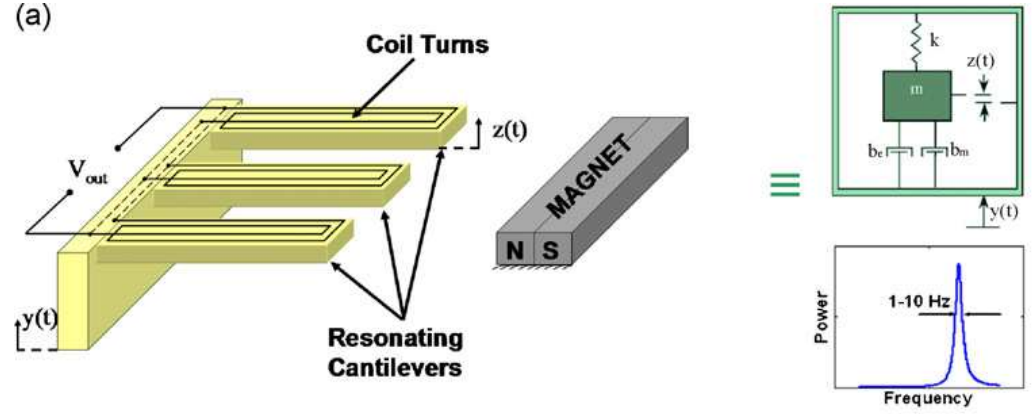

(b)
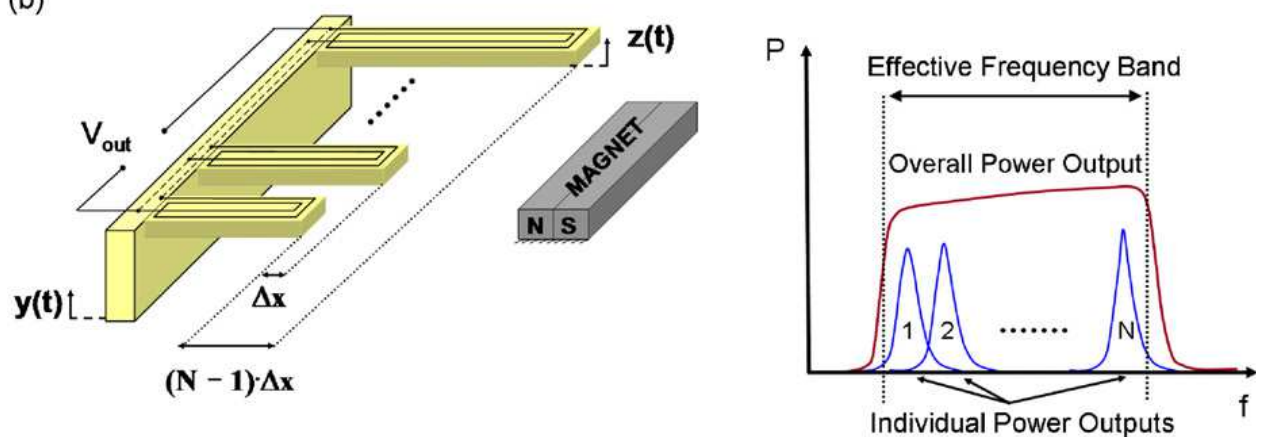

Figure 1.20: Schematic expected responce of a power generator with (a) only one resonance frequency and multiple resonance frequencies developed by Sari et al. Reprinted from [45], Copyright (2008), with permission from Elsevier.

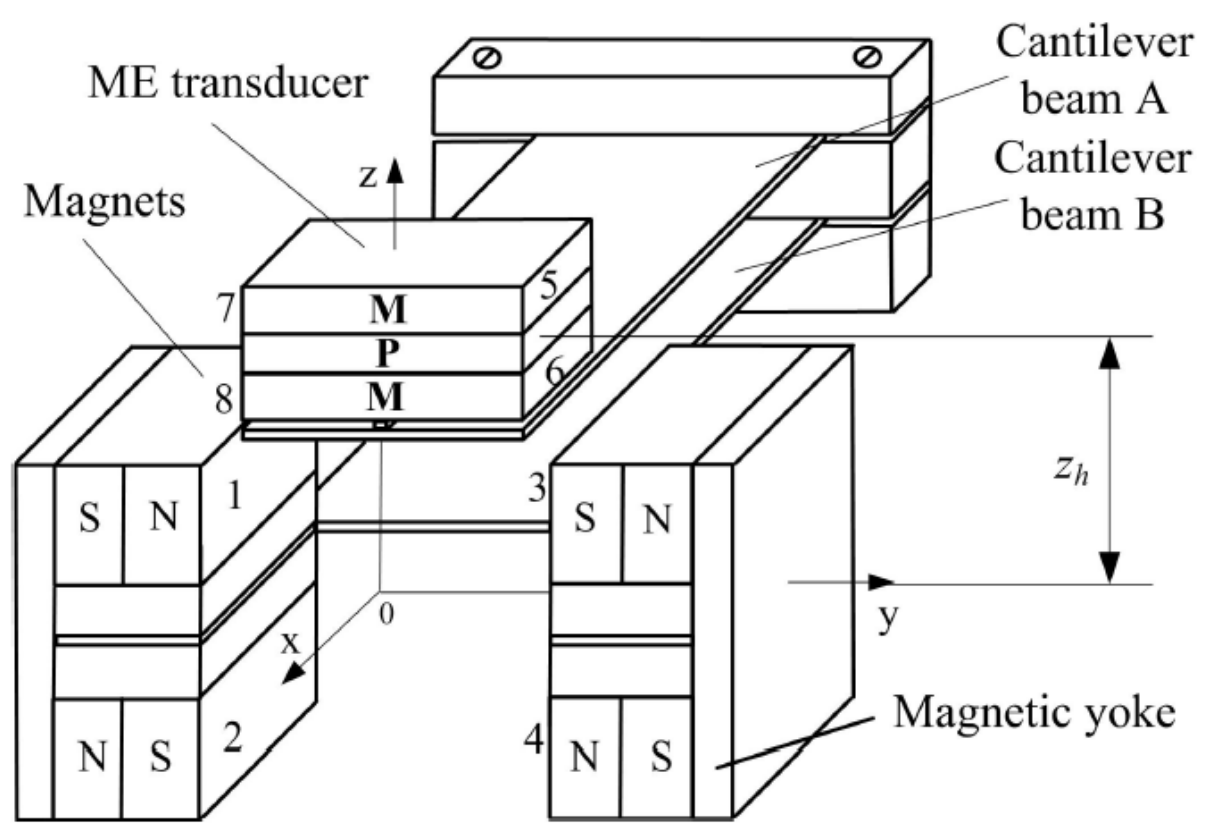

Figure 1.21: Schematic developed by Yang et al. (C)[2010] IEEE. Reprinted, with permission, from [39]. 


\subsection{Magnetoelectric modeling}

Different models had been proposed to predict the magnetoelectric response in composite structures. All these models predict the magnetoelectric coupling between the piezoelectric and piezomagnetic phase through mechanical coupling. These models are implemented analytically and numerically using different materials, geometries, and methods.

An important property of the magnetoelectric composites is the electric conductivity. The conductivity of the composites has to be low in order to maintain the voltage generated through the thickness or collect the charge. When the conductivity of the composite is excessively high, the magnetoelectric effect cannot be measured. In the case of a laminate structure, the conductivity of the composite can be easily calculated and by proper manufacture can be kept low. In the case of particulate composites, to keep the conductivity low is a challenge due to the high conductivity of the magnetic phase. Up to now there is no model that can predict the conductivity of these particulate composites. This will be further discussed in Chapter 4.

\subsubsection{Analytical models}

\section{Harshe1993}

The first analytical model of magnetoelectric composites was the one reported by Harshé et al. [46]. In here the constitutive equations used are the piezoelectric effect:

$$
\begin{aligned}
D & =d \sigma \\
E & =g \sigma \\
g & =-\frac{d}{\epsilon^{T}}
\end{aligned}
$$

where $D$ is the electric displacement, $d$ is the piezoelectric coefficient, $\sigma$ is the stress, $E$ is 
the electric field, $g$ is the piezoelectric voltage coefficient and $\epsilon$ is the dielectric permittivity. The constitutive equations for the magnetostrictive phase at low magnetic fields and assuming no hysteresis con be expressed as follows:

$$
s=\lambda H^{2}
$$

where $s$ is the strain, $\lambda$ is the magnetostriction coefficient and $H$ is the magnetic field. In this work the magnetostriction was taken as pseudo-piezomagnetic, which means that near a given magnetic field, $H_{0}$, the slope of the magnetostriction curve can be assumed to be constant. This pseudo-piezomagnetism at a bias field $H_{0}$ can be expressed as:

$$
s=k H
$$

where $k$ is the pseudo-piezomagnetic coefficient. These constitutive equations were used to model a laminate structure, 2-2 composite, using $\mathrm{CoFe}_{2} \mathrm{O}_{4}$ as magnetostrictive phase and $\mathrm{BaTiO}_{3}$ and PZT as piezoelectric phase. Harshé et al. calculated the magnetoelectric voltage coefficient for different thickness of the piezoelectric and piezomagnetic phase [46]. In order to obtain the optimum performance a compromise between the thicknesses has to be made. In addition to this they studied the influence of the dielectric constant in the magnetoelectric coefficient showing a decrease of the magnetoelectric coefficient when materials with higher dielectric constants are used.

\section{Harshe1993a}

In the second publication by Harshé et al. the same constitutive equations were used to predict the magnetoelectric voltage coefficient on a 0-3 and 3-0 (piezoelectric-piezomagnetic) composite structure. This structure consist of cubed particles (piezoelectric or piezomagnetic) 
in a matrix (piezomagnetic or piezoelectric). The properties used for each phase are $\mathrm{CoFe}_{2} \mathrm{O}_{4}$, BTO and PZT. They found that the theoretical magnetoelectric coefficient is one order of magnitude higher than the laminate structure previously reported. In addition, the composite structure was fabricated using solid state sintering. The experimental results obtained showed that the measured magnetoelectric coefficient is two orders of magnitude lower than the calculated. This is attributed to the high conductivity given by the connected conductive particles.

\section{Nan1994}

In the work of Nan in 1994, the interaction between the piezoelectric and piezomagnetic phases in a magnetoelectric composite were studied [47]. The piezoelectric and piezomagnetic phases have linear coupling between the electric fields and deformation and between the magnetic fields and deformation, respectively. In [47] the magnetic phase is assumed to have a linear response, piezomagnetic, with the applied magnetic fields.

The work presented by Nan models the magnetoelectric effect by the mechanical coupling between the piezoelectric and piezomagnetic phase described by the equation 1.8. The constitutive equations used to couple the magnetic-electrical-mechanical interactions in each phase are:

$$
\begin{aligned}
\sigma & =C s-e^{T} E-q^{T} H \\
D & =e s+\epsilon E+\alpha H \\
B & =q s+\alpha^{T} E+\mu H
\end{aligned}
$$

where $\sigma, s, E, H, D, B$ are the stress tensor, strain tensor, electrical field, magnetic field, electrical displacement and magnetic induction, respectively. $C, \epsilon$ and $\mu$ are the stiffness tensor (measured at constant electrical and magnetic field) and dielectric and magnetic per- 
meability tensors (measured at constant strain), respectively. $e, q$ and $\alpha$ are the piezoelectric, piezomagnetic and magnetoelectric coefficient tensors, respectively. Of these tensors, $C$ is a forth-rank tensor; $e$ and $q$ are third-rank tensors; $\epsilon, \mu, \alpha, s$ and $\sigma$ are second-rank tensors; $E, D, H$ and $B$ are first-rank tensors.

The piezoelectric phase does not present piezomagnetic or magnetoelectric coupling. This means that $q=0$ and $\alpha=0$ reducing the equation 1.15 to:

$$
\begin{aligned}
\sigma & =C s-e^{T} E \\
D & =e s+\epsilon E \\
B & =\mu H
\end{aligned}
$$

The piezomagnetic phase does not present piezoelectric or magnetoelectric coupling $(e=0$ and $\alpha=0)$, reducing equation 1.15 to:

$$
\begin{aligned}
\sigma & =C s-q^{T} H \\
D & =\epsilon E \\
B & =q s+\mu H
\end{aligned}
$$

The mechanical transfer of the stress and strain between these phases gives as a result magnetoelectric coupling in the composite structure, which is not present in the constituent phases. In the work presented by Nan the magnetoelectric coupling of the composite was calculated by using the corresponding boundary conditions and geometry. In this work two different geometries were used 1-3 and 3-1 (piezoelectric-piezomagnetic) structures. In the case of the 1-3 structure the piezoelectric is connected in one axis and the piezomagnetic in the three axis, which represents piezoelectric fibers in a piezomagnetic matrix. And the case 
of the 3-1 piezoelectric-piezomagnetic the piezoelectric phase is connected in one axis and the piezomagnetic phase in the three axis. This geometry would not allow the piezoelectric phase to generate an electric field, or electric displacement, in practical applications due to the fact that the conductivity of the composite would be too high. The conductivity of the magnetic phase is several orders of magnitude higher than the piezoelectric phase (Conductivity of Terfenol-D, $\mathrm{CoFe}_{2} \mathrm{O}_{4}$ and PZT are $10^{4}, 10^{-2}$ and $\left.10^{-8} \mathrm{~S} / \mathrm{cm}\right)$. In this model the effect of the conductivity in the magnetoelectric performance is not evaluated, making it unrealistic.

The magnetoelectric coupling can be defined by measuring the generated voltage or the charge when a magnetic field is applied. These different coefficients are called magnetoelectric voltage coefficient and magnetoelectric charge coefficient. In order to measure the voltage the circuit between the electrodes has to be open to prevent the capacitor to be discharged and the voltage between electrodes has to be measured. The magnetoelectric charge coefficient is measured by using a closed circuit and measuring the charge, or current, that is generated as a response of the applied magnetic field. When these parameters are calculated using models the corresponding boundary conditions have to be applied. After one coefficient is calculated the other one can be calculated using the following equation:

$$
\alpha=-\frac{\beta}{\epsilon}
$$

where $\alpha$ is the magnetoelectric voltage coefficient, $\beta$ is the magnetoelectric charge coefficient, and $\epsilon$ is the dielectric constant of the composite in the direction of the electric field or charge displacement.

The results obtained by Nan show a compromise between the amount of the piezoelectric and piezomagnetic phase. The optimum volume fraction is different for the case of magnetoelectric voltage coefficient and the magnetoelectric charge coefficient. That is why depending on the application, where voltage or charge will be measured, different volume fractions have to be used to optimize the output. In [47] shows that the aspect ratio has a large influence 
in the magnetoelectric coupling. The magnetoelectric coupling shows an increase when the fibers have a higher aspect ratio.

\section{Osaretin2010}

An analytical model to calculate the magnetoelectric coupling in laminate composites was made by Osaretin et al. [48]. In [48] the constitutive equations are slightly different than ecuation 1.15 , i.e., using stress, $\sigma$, as independent variable rather than strain, $s$ :

$$
\begin{aligned}
s & =S \sigma+d E+q H \\
D & =d \sigma+\epsilon E+\alpha H \\
B & =q \sigma+\alpha^{T} E+\mu H
\end{aligned}
$$

where $S$ is the compliance, $d$ is the piezoelectric constant, $q$ is the piezomagnetic constant, $\epsilon$ is the electric permittivity and $\mu$ is the magnetic permeability. In [48] two main modifications were made in comparison to the work made by Harshé et al.. First, they introduced the use of coupling parameter, $k$. Second, they applied different boundary conditions.

The coupling parameter, $k$, is used to model the non perfect mechanical interaction in the boundary. This coupling parameter is defined as:

$$
k=\frac{s^{e}}{s^{m}}
$$

where the superscript $e$ and $p$ represents for the piezoelectric and piezomagnetic phase, respectively. This assumes that the strain induced in one phase may not be completely transfered to the other phase due to several factors like mechanical defects, bonding material, etc. These factors are contained and described by the interface coupling parameter. This parameter cannot be measured experimentally. In some works this parameter is used to fit 


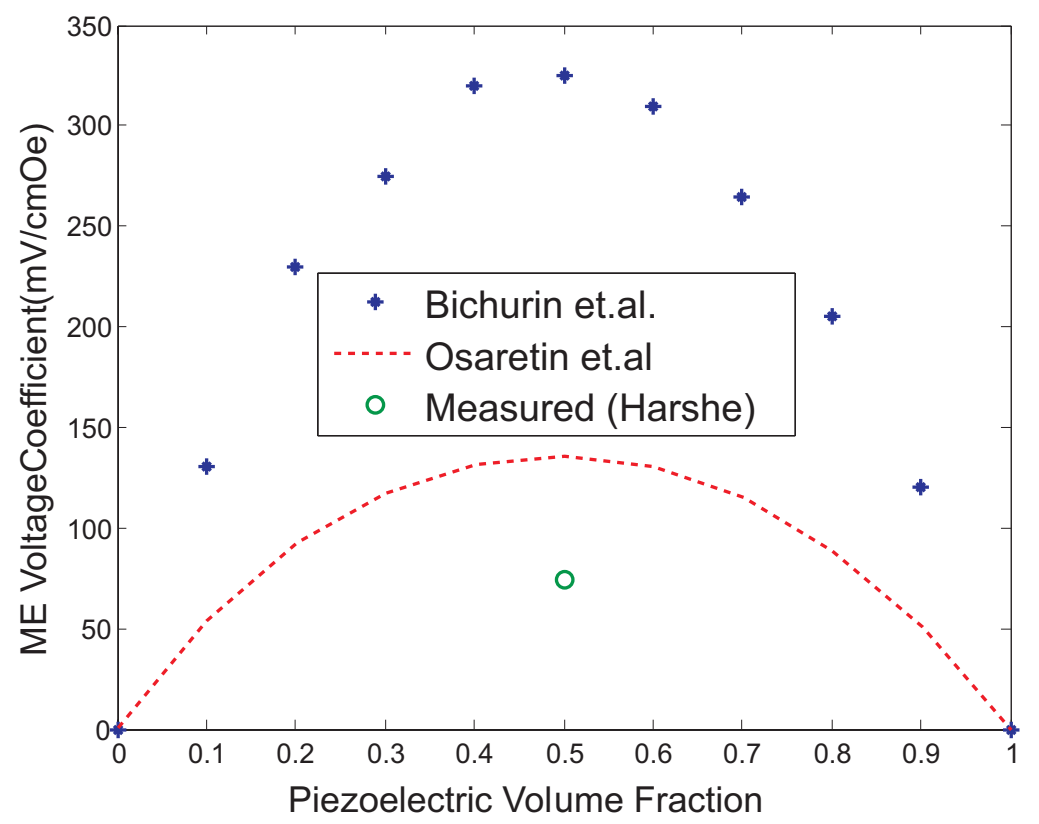

Figure 1.22: Comparison of the analytical model made by Bichurin et al., Osaretin et al. $(k=1)$ and measured values by Harshé $[49,48,46]$. Reprinted with permission from [48]. Copyright (2010) by the Americal Physical Society.

the model to the experimental data by choosing the corresponding coupling parameter. This coupling parameter will not be taken into account in Chapter 2 and Chapter 3 because it is a fitting parameter which cannot be experimentally measured or proven.

The use of more realistic boundary conditions in this work showed that the results obtained by this model are closer to the experimental results than those of Bichurin and Harshé $[49,46]$. This can be observed in Figure 1.22, were it can be seen that the work made by Osaretin et al. is closer to the experimental data than the work made by Bichurin et al.

\subsubsection{Numerical models}

\section{Sun2013}

Sun et al. calculated the magnetoelectric coupling of composite structures employing COMSOL software [50]. In this work the piezoelectric phase is modeled using a linear relation between the strain and the electric fields but in the case of the magnetic phase quadratic relation between the applied magnetic fields and the strain was used (similar to equation 1.13). 
In order to calculate the magnetoelectric coefficient the model was made in two steps. First the magnetostrictive equations were solved and with the obtained results the piezoelectric system was analyzed. This process was repeated until the system converged.

Two different geometries were studied in this work, 1-3 and 3-0. The 1-3 geometry consisted in 9 piezoelectric rods embedded in a magnetic matrix. This configuration electrically connects the electrodes through the magnetic phase, since the conductivity of the magnetic phase is several orders of magnitude higher than the piezoelectric phase. In this case no voltage or charge could be measured, i.e., all the charge would leak through the magnetic phase. In the case of the 3-0, the geometry consists in spheres of the magnetic phase in a piezomagnetic matrix. The geometry was made with 125 spheres randomly distributed, using Monte Carlo method. In this work only one volume fraction of $12.5 \%$ magnetic phase was used. In order to obtain an accurate result, 100 different random geometries were randomly made, calculated and averaged. The materials properties used for these models were the corresponding to PZT - 5H and $\mathrm{CoFe}_{2} \mathrm{O}_{4}$. The results obtained for the 3-0 geometry can be seen in Figure 1.23.

\section{Bouillault}

The magnetoelectric coupling on laminated structures was studied by Bouillault et al. for sensors applications $[51,52,53,54]$. The model implemented on COMSOL following a similar convergence method as Sun et al.. This model was used to estimate the voltage response of a magnetic sensor using as sensing material a laminate magnetoelectric composite. In this work the performance of the sensor was measured but the magnetoelectric coupling of the composite was not measured or discussed. 

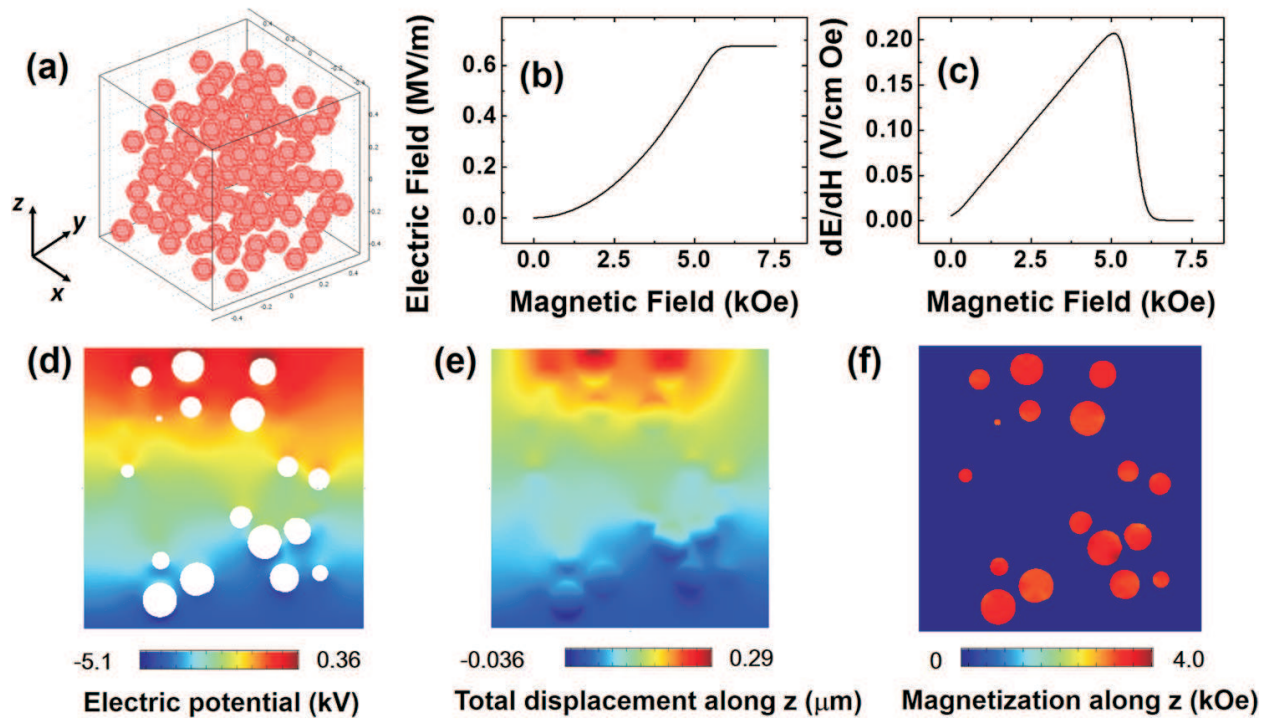

Figure 1.23: Results obtained by Sun et al.; (a) particles distributed in a matrix randomly, (b) output electric field as a response of the applied magnetic field, (c) $\mathrm{dE} / \mathrm{dH}$ as a funtion of the input magnetic field, (d) Distribution of the electric potential, (e) total displacement and (d) magnetization. Reprint with permission from [50]. Copyright [2013], AIP Publishing LLC. 


\section{Chapter 2}

\section{Charge, voltage, and work-conversion formulas for magnetoelectric}

\section{laminated composites}

A novel analytical model for magnetoelectric (ME) laminate composites made of piezoelectric $(\mathrm{PE})$ and piezomagnetic $(\mathrm{PM})$ phases is proposed ${ }^{1}$. The multiphysics equations are applied to all four possible laminate configurations (TT, LT, TL, and LL), with appropriate boundary conditions. Closed form, explicit formulas are derived for the calculation of the intrinsic ME charge coefficient, ME voltage coefficient, and ME coupling factor as a function of material properties of both phases and the PM volume fraction. The predicted ME voltage coefficient is in agreement with previous work and experimental data. A new approach is proposed to take into account the conductivity of the PM phase resulting in calculated ME charge coefficients within $30 \%$ of experimental data, which is a major departure from the available approaches that either require to impose an additional constraint on the model or simply ignore the conductivity of the PM phase. To assess the conversion of magnetic work into electric work, a novel approach is developed to calculate the ME coupling factor in closed

\footnotetext{
${ }^{1}$ This chapter was published in Smart Materials and Structures journal [55]. (c) IOP Publishing. Reproduced with permission. All rights reserved.
} 
form by using the calculated properties of the ME composite structure, thus avoiding the equivalent circuit assumption, and furthermore novel coupling factor formulas are developed for all four polarization/magnetization configurations and taking into account the strain coupling in both in-plane directions. Using actual material properties, conclusions are drawn regarding the optimal configuration and PM volume fraction necessary to achieve maximum charge, voltage, and work conversion.

\section{$2.1 \quad$ Introduction}

Interest in magnetoelectic (ME) devices is motivated by potential applications such as sensors [29], energy harvesters [42, 44, 41, 32, 45, 43, 40], and solid state memory [56]. Although ME materials exist in nature [6], their ME charge coefficient $\beta$ is too small $\left(4.13 p s m^{-1}{ }^{2}\right.$ at $2^{\circ} \mathrm{K}$ [10]) and/or their Néel temperature is too low for practical applications.

On the other hand, composite materials can be built to exploit the product property between a strongly magnetostrictive (MS) $s / H$ material and a strongly piezoelectric (PE) $E / s$ material; that is, obtaining the product ME property $E / H$, where $s, H, E$, are the strain, magnetic filed, and electric field, respectively. Unlike naturally occurring ME materials, ME composites can achieve very strong ME voltage coefficients $\left(\alpha=5.76 \mathrm{~V}^{-1}{ }^{3}\right.$ [22], which corresponds to $\beta \sim 13000 \mathrm{ps} \mathrm{m}^{-1}$ ) at room temperature. Therefore, ME composites are considered to be metamaterials [57].

While particulate composites are limited in performance by atomic diffusion, mechanical defects, and leakage currents, laminated composites may overcome or minimize these problems $[22,33,58]$. Since the constituent PE and MS materials, and the laminated composite they form, all have other useful properties such as the ability to carry loads (stiffness and strength), ME composites may be considered to be multifunctional materials as well [59].

PE materials display an approximately linear strain-electric field response over a wide

\footnotetext{
${ }^{2} C A^{-1} m^{-1}=s m^{-1}$

${ }^{3} \mathrm{VA}^{-1}=796 \mathrm{mVcm} \mathrm{cm}^{-1} \mathrm{Oe}$
} 
range of strain or electric fields. On the other hand, MS materials display a nonlinear strainmagnetic field $(\mathrm{s}-\mathrm{H})$ response. However, most applications use these materials over a small magnetic-field range centered around a large, fixed magnetic bias. As a result, the behavior may be approximated as linear, and in this later case the material is said to be piezomagnetic $(\mathrm{PM})$.

Analytical models $[46,47,48,49]$ are useful because they can be used to quickly predict approximate values for the expected voltage, charge, or work conversion of the device as a function of material properties for the PE and PM materials as well as a function of the relative thicknesses, or volume fractions of the constituents. Therefore, an analytical model for ME laminate composites made of PE and PM layers is developed herein. The governing equations are applied to all four possible laminate configurations (TT, LT, TL, and LL), with appropriate boundary conditions. The system of equations is solved using Wolfram Mathematica [60]. Conclusions are drawn regarding the merits, drawbacks, and requirements for analysis of each configuration.

Closed form, explicit formulas are derived for the calculation of the ME voltage coefficient, $\alpha$, as a function of the material properties of PE and PM phases, and the PM volume fraction, $\chi$. The predicted ME voltage coefficient is in agreement with previous work and experimental data.

Also, closed form, explicit formulas are derived for the calculation of the ME charge coefficient $\beta$ as a function of the material properties of PE and PM phases, and the PM volume fraction. The values of ME charge coefficient previously calculated in the literature do not agree with available experimental data. This is due to the fact that the high conductivity of the PM phase has not being taken into account in previous models [48], giving predictions three orders of magnitude smaller than experimental values. A new approach is proposed in Section 2.2.2 to take into account the conductivity of the PM phase, resulting in calculated ME charge coefficients within $30 \%$ of experimental data. The new proposed approach does not require to impose the additional constraint of zero electric field in the PM. While this 
would be acceptable for the particular case of laminates, it would not apply to more general cases. For example, such constraint would imply that a finite element implementation for arbitrary shape of PE inclusions in a PM matrix would have to be programmed with two different element formulations; one with the correct constitutive equation (2.2) for the $\mathrm{PE}$ phase and another with $\mathrm{E}=0$ for the PM phase, thus canceling (2.2). To avoid such inconsistency and lack of generality, a new approach is proposed within the context of electrostatics, magnetostatics, and elasticity; that is, without requiring the addition of electrodynamics, which would increase the complexity of the constitutive equations significantly.

The ME voltage coefficient provides a indication of the ME performance to produce an electric field $E$, and thus voltage $V=E t$ from exposure to a magnetic field $H$, when no work is drawn from the device ${ }^{4}$. This definition may lead to maximum ME voltage performance when the volume fraction of PM phase $\chi \rightarrow 1$, but a nearly zero thickness of PE would produce almost no work and thus make it difficult to measure the voltage.

Similarly, the ME charge coefficient provides an indication of the ME performance to produce an electric displacement $D$, and thus charge $Q=D A$ (where $A$ is the area of the device) from exposure to a magnetic field $H$, again when no work is drawn from the device. This definition may lead to maximum ME charge performance when the volume fraction of PM phase $\chi \rightarrow 1$, but a nearly zero thickness of PE would produce almost no work and thus make it very difficult to measure the charge $Q$ or current $I=\int Q d t$.

The ME coupling factor $\kappa$ provides a indication of the ME performance to convert magnetic work into electric work [61,62]. This coefficient has been calculated in [61] only for the push-pull configuration, and only the mechanical coupling in the direction of the applied magnetic field was considered. Furthermore, an equivalent circuit for the composite had to be assumed in [61]. In this work, closed form, explicit formulas are derived for the calculation of the ME coupling factor as a function of the material properties of PE and PM phases, and the PM volume fraction considering mechanical coupling in all directions, without the need

\footnotetext{
${ }^{4}$ Where $t$ is the total thickness of the device, $E$ is the average electric field over $t$, and $V$ is the voltage measured across the thickness $t$ of the device.
} 
to assume an equivalent circuit.

The formulas for these coefficients require calculation of the dielectric permittivity of the device $\epsilon^{H}$ at constant magnetic field, $H$, and magnetic permeability of the device $\mu^{E}$ at constant electric field, E. The well known series/parallel capacitor formulas are not appropriate for PM devices because the application of a magnetic field results in an electrical displacement and vice versa, due to the inherent coupling present in the device. Thus, a methodology for the calculation of these coefficients is proposed, which is then used to derive close form, explicit formulas for their calculation.

This work is concerned with predicting the intrinsic material properties of the composite, while the extrinsic properties are those that can be measured in an experimental setup. The difference between intrinsic and extrinsic can be calculated by established formulas for the demagnetizing effect $[63,64]$.

Three materials, LSMO/PZT, FeBSiC/PZN-PT, and Terfenod-D/PZT-5H are used to illustrate salient aspects of the model, of the various configurations, and of the three coefficients. Application of the proposed formulas highlights a trade off between deformation and charge/voltage/work as a function of PM volume fraction. Further use of these formulas for optimization is delayed until Chapter 3.

\subsection{Model description}

The analytical model is based in the following constitutive equations:

$$
\begin{aligned}
s & =S \sigma+d^{T} E+q^{T} H \\
D & =d \sigma+\epsilon E \\
B & =q \sigma+\mu H
\end{aligned}
$$

where $\sigma$ is the stress tensor, $s$ is the strain tensor, $E$ is the electric field vector, $H$ is the magnetic field vector, $D$ is the electric displacement vector, $B$ is the magnetic flux density 
vector, $S$ is the compliance tensor (measured at constant electric and magnetic field), $\epsilon$ is the dielectric permittivity tensor (measured at constant stress), $\mu$ is the magnetic permeability tensor (measured at constant stress), $d$ is the $\mathrm{PE}$ charge constant tensor, and $q$ is the $\mathrm{PM}$ constant tensor. These equations describe the behavior of the PM and PE phases. As it was discussed before, all the materials used as a PM are actually magnetostrictive with a nonlinear relationship between strain and magnetic field, but they are customarily treated as linear in the close neighborhood of an applied magnetic bias. Then, the PM constant tensor and all the PM properties are calculated at this magnetic bias.

In this work the materials are assumed to be transversally isotropic. For PE and PM materials the axis of symmetry is the direction at which the material is polarized or magnetized, respectively. However, for sake of expedience, this direction is simply called the polarization (or magnetization) direction. Further, it is convenient to define a material coordinate system were the 3-direction is aligned with the polarization (or magnetization), see Figure 2.1. Since each phase can be polarized, or magnetized, in different directions, the global coordinate system of the composite may not coincide with the material coordinate system.

The compliance tensor $S$ is then defined as follows (Equation 1.92 from [?]):

$$
S=\left[\begin{array}{cccccc}
S_{11} & S_{12} & S_{23} & 0 & 0 & 0 \\
S_{12} & S_{11} & S_{23} & 0 & 0 & 0 \\
S_{23} & S_{23} & S_{33} & 0 & 0 & 0 \\
0 & 0 & 0 & S_{44} & 0 & 0 \\
0 & 0 & 0 & 0 & S_{44} & 0 \\
0 & 0 & 0 & 0 & 0 & 2\left(S_{11}-S_{12}\right)
\end{array}\right]
$$




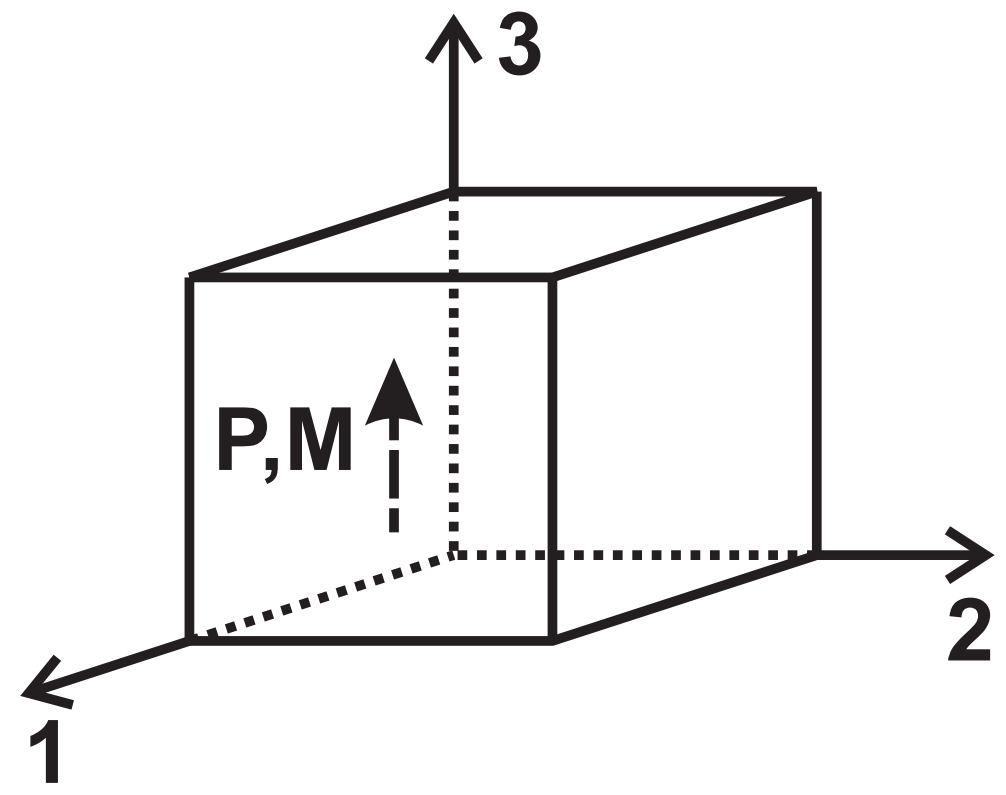

Figure 2.1: Material coordinate system.

The PE charge constant tensor $d$ is defined as

$$
d=\left[\begin{array}{cccccc}
0 & 0 & 0 & 0 & d_{15} & 0 \\
0 & 0 & 0 & d_{15} & 0 & 0 \\
d_{31} & d_{31} & d_{33} & 0 & 0 & 0
\end{array}\right]
$$

where

$$
\left.\left.d_{i j}=\frac{s_{j}}{E_{i}}\right]_{\sigma=0}=\frac{D_{i}}{\sigma_{j}}\right]_{E=0}
$$

The PM constant tensor $q$ is defined in the same way, where

$$
\left.\left.q_{i j}=\frac{s_{j}}{H_{i}}\right]_{\sigma=0}=\frac{B_{i}}{\sigma_{j}}\right]_{H=0}
$$

The dielectric permittivity $\epsilon$, and magnetic permeability $\mu$, are diagonal tensors defined 


$$
\begin{gathered}
\epsilon=\epsilon_{i j} \delta_{i j} \\
\mu=\mu_{i j} \delta_{i j}
\end{gathered}
$$

where $\delta_{i j}$ is the Kronecker symbol and $x_{3}$ is the axis of transverse isotropy, which coincides with the direction of polarization or magnetization $\left(\epsilon_{11}=\epsilon_{22}\right.$ and $\left.\mu_{11}=\mu_{22}\right)$.

ME devices can be built using four different configurations (Figure 2.2): transverse magnetization with transverse polarization (TT), longitudinal magnetization with transverse polarization (LT), transverse magnetization with longitudinal polarization (TL), and longitudinal magnetization with longitudinal polarization (LL). For simplicity, only two layers are shown in Figure 2.2, but it is assumed that the actual device is symmetrically laminated. Furthermore, the laminas are thin in comparison to the in-plane dimensions, resulting in a state of plane stress [65] and insignificant shear lag effect [63]. As a result, the stresses considered in this work are averaged through the thickness of each lamina and the intralaminar/interlaminar shear strains are negligible [65]. Consequently, the ME pair is assumed to be fully effective over its entire area.

The PE phase is most commonly polarized in the transverse direction. Otherwise, an insulator is needed at the interface to prevent charge leakage from the PE through the PM phase, since the latter is highly conductive. For example, a longitudinally polarized PZT was bonded to two magnetostrictive FeBSiC alloy (Metglas) foils, using two Kapton films in between to avoid leakage [66].

The four possible geometric configurations shown in Figure 2.2 (a), (b), (c) and (d) are implemented by appropriate boundary conditions, as follows. 

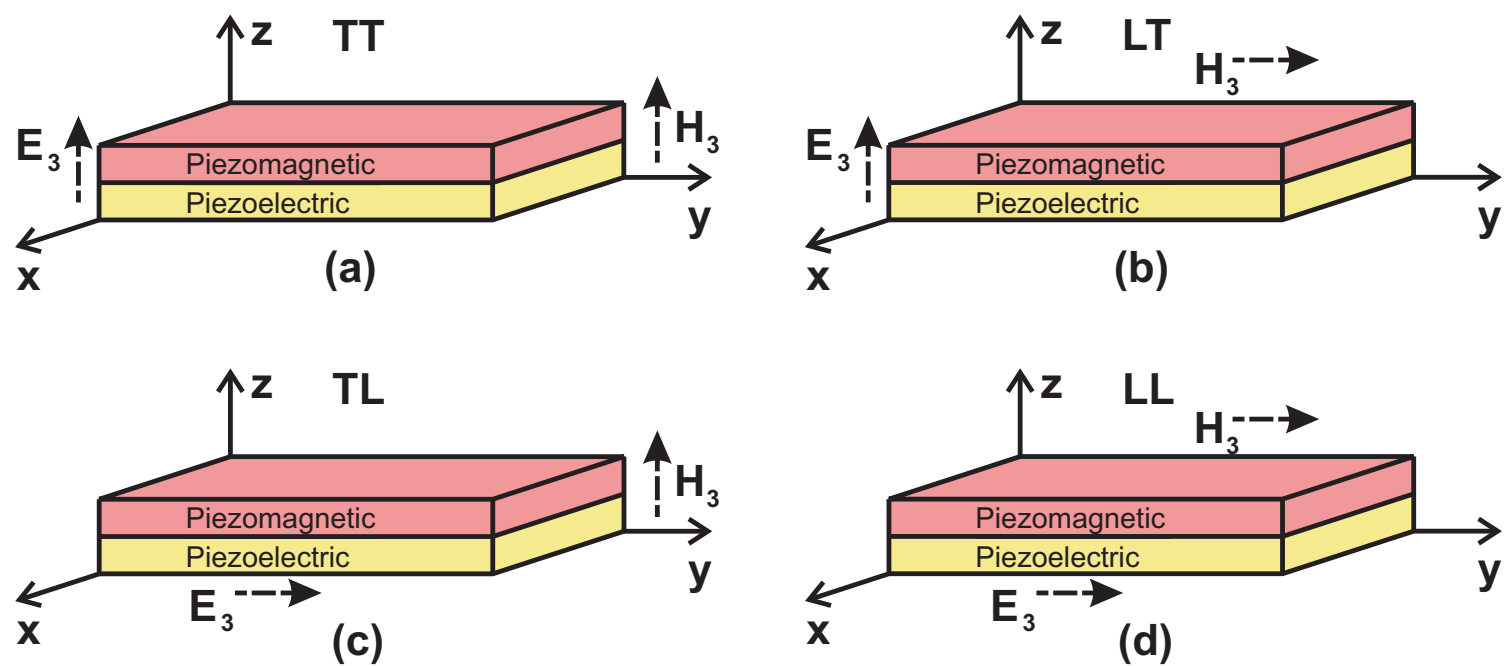

Figure 2.2: Laminate configurations: (a) transverse magnetization and transverse polarization (TT), (b) Longitudinal magnetization and transverse polarization (LT), (c) transverse magnetization and longitudinal polarization (TL), and (d) longitudinal magnetization with longitudinal polarization (LL).

\subsubsection{Transverse magnetization and polarization (TT)}

Using (2.1) for the first term of the strain vector, yields

$$
s_{x}=\sigma_{x}\left(S_{11}+S_{12}\right)+d_{31} E_{z}+q_{31} H_{z}
$$

where $\sigma_{x}=\sigma_{y}$ because of the symmetry of the applied fields and geometry, $\sigma_{z}=0$ because the laminate is not restrained to deform in the z-direction. Also, PE and PM are polarized (magnetized) in the z-direction shown in Figure 2.2.a. Equation (2.10) is valid for both PM and PE phases. Since $d_{31}^{P M}=0$ in the PM phase, (2.10) reduces to

$$
s_{x}^{P M}=\sigma_{x}^{P M}\left(S_{11}^{P M}+S_{12}^{P M}\right)+q_{31}^{P M} H_{z}^{P M}
$$

Since $q_{31}^{P E}=0$ in the PE phase, (2.10) reduces to

$$
s_{x}^{P E}=\sigma_{x}^{P E}\left(S_{11}^{P E}+S_{12}^{P E}\right)+d_{31}^{P E} E_{z}^{P E}
$$


From (2.2), the electric displacement in the z-direction, $D_{z}$, is expressed as

$$
D_{z}=2 d_{31} \sigma_{x}+\epsilon_{33} E_{z}
$$

Since $d_{31}^{P} M=0$ in the PM phase

$$
D_{z}^{P M}=\epsilon_{33}^{P M} E_{z}^{P M}
$$

and for the PE phase

$$
D_{z}^{P E}=2 d_{31}^{P E} \sigma_{x}^{P E}+\epsilon_{33}^{P E} E_{z}^{P E}
$$

Using the same approach the magnetic flux density in the z-direction for the PM phase is

$$
B_{z}^{P M}=2 q_{31}^{P M} \sigma_{x}^{P M}+\mu_{33}^{P M} H_{z}^{P M}
$$

and the magnetic flux density for the PE phase is

$$
B_{z}^{P E}=\mu_{33}^{P E} H_{z}^{P E}
$$

Assuming that the PE and PM layers are perfectly bonded, the strain in the $\mathrm{x}$ and y-direction on both layers are equal

$$
s_{x}^{P E}=s_{x}^{P M}
$$

By force equilibrium, the force in the PM and PE phases have the same magnitude and opposite direction. The PM volume fraction, $\chi$, is defined to take into account laminae with 
different thicknesses

$$
\chi=\frac{V^{P M}}{V^{P M}+V^{P E}}
$$

where $V$ is the volume of each phase. Then, the equilibrium of forces is expressed as

$$
\sigma_{x}^{P M}=-\sigma_{x}^{P E}\left(\frac{1}{\chi}-1\right)
$$

To produce an electric displacement, $D$, charge has to flow in the laminate of a certain area. Since the charge can only flow from the top to the bottom electrode, the electric displacement, $D$, is the same in the PE and PM phases

$$
D_{z}^{P M}=D_{z}^{P E}
$$

The same applies to the magnetic flux density

$$
B_{z}^{P M}=B_{z}^{P E}
$$

Since the magnetic and electric fields that are externally measured are average values, it is convenient to define the following averages

$$
\begin{aligned}
E^{a v g} & =E_{z}^{P E}(1-\chi)+E_{z}^{P M} \chi \\
H^{a v g} & =H_{z}^{P E}(1-\chi)+H_{z}^{P M} \chi
\end{aligned}
$$

The ME voltage coefficient is experimentally measured by applying a magnetic field to the composite and measuring the open circuit voltage $\left(D_{z}=0\right)$. The ME voltage coefficient, $\alpha$, is an intrinsic property of the composite and is calculated by dividing the measured voltage 
by the thickness and the applied magnetic field. This can be mathematically expressed as

$$
\left.\left.\alpha=\frac{V}{t H^{\text {avg }}}\right]_{D=0}=\frac{E^{a v g}}{H^{\text {avg }}}\right]_{D=0}
$$

where $V$ is the voltage generated between the upper and lower electrode as a response of the applied magnetic field and $t$ is the total thickness of the composite. The second equality is obtained by the definition of the electric field $\left(E=\frac{V}{t}\right)$. In this work, the ME voltage coefficient for the TT configuration is calculated by solving the system of equations (2.11)(2.23), resulting in the following formula

$$
\begin{aligned}
\alpha_{T T}= & \frac{A_{1}}{B_{1}} \\
A_{1}= & -\left(2 d_{31}^{P E} \mu_{33}^{P E} q_{31}^{P M}(\chi-1) \chi\right) \\
B_{1}= & \left(2\left(d_{31}^{P E}\right)^{2} \chi\left(\mu_{33}^{P M}+\mu_{33}^{P E} \chi-\mu_{33}^{P M} \chi\right)+\epsilon_{33}^{P E}\left(2\left(q_{31}^{P M}\right)^{2}(\chi-1)^{2}\right.\right. \\
& \left.\left.-\left(\mu_{33}^{P M}(\chi-1)-\mu_{33}^{P E} \chi\right)\left(S_{11}^{P M}(\chi-1)+S_{12}^{P M}(\chi-1)-\left(S_{11}^{P E}+S_{12}^{P E}\right) \chi\right)\right)\right)
\end{aligned}
$$

The ME voltage coefficient calculated in this work is compared to the results reported in [49]. Lead zirconate titanate (PZT) and lanthanum strontium manganite (LSMO) were used in [49] for the PE and PM phase, respectively. The properties used for these materials are reported in Table 2.1. The results obtained from the model proposed in [49] for different PM volume fractions is compared with the proposed model. The outcome can be seen in Figure 2.3, showing agreement between the models. The data shows a trade off between the volume of $\mathrm{PE}$ and PM phase with an optimum value of ME voltage coefficient at $\chi=0.65$.

When higher volumes of PM phase are used (higher PM volume fraction $\chi$ ), it gives higher strain, resulting in a higher electric field in the PE phase. The relationship between the electric field in the PE phase and the electric field in the composite can be seen in (2.21), showing that the total electric field depends on the electric field produced in the PE phase and the PM volume fraction (or thickness).

For PM volume fraction below the optimum, increasing the thickness of the PM phase 


\begin{tabular}{c||c|c||c} 
Material Property & LSMO & PZT & Units \\
\hline \hline$S_{11}$ & 15 & 15.3 & $10^{-12} m^{2} N^{-1}$ \\
\hline$S_{12}$ & -5 & -5 & $10^{-12} m^{2} N^{-1}$ \\
\hline$\epsilon_{33} / \epsilon_{0}$ & 10 & 1750 & \\
\hline$\mu_{33} / \mu_{0}$ & 2 & 1 & \\
\hline$d_{31}$ & 0 & -175 & $10^{-12} C N^{-1}$ \\
\hline$d_{33}$ & 0 & 400 & $10^{-12} C N^{-1}$ \\
\hline$q_{31}$ & 250 & 0 & $10^{-12} m A^{-1}$ \\
\hline$q_{33}$ & -120 & 0 & $10^{-12} m A^{-1}$ \\
\hline
\end{tabular}

Table 2.1: Material Properties of PZT and LSMO from [49].

gives an increase of the total electric field, which is stronger than the effect of the reduction of the thickness of the PE phase. But in the case of PM volume fraction above the optimum, even though the electric field in the PE phase increases with the increased thickness of PM phase, the decrease in PE phase thickness results in a decrease of the total electric field of the composite. Thus the parabolic response observed in Figure 2.3.

The ME charge coefficient $\beta$ is experimentally measured by applying a magnetic field to the composite and measuring the short circuit charge with $E=0$. The ME charge coefficient is an intrinsic property that is calculated as follows

$$
\left.\left.\beta=\frac{Q}{A H^{a v g}}\right]_{E=0}=\frac{D^{a v g}}{H^{a v g}}\right]_{E=0}
$$

where $Q$ is the charge generated in the direction of polarization and $A$ is the total area of composite perpendicular to the polarization.

An analytical expression for the ME charge coefficient in the TT configuration is obtained by solving the previous system of equations with the average electric field equal to zero, 


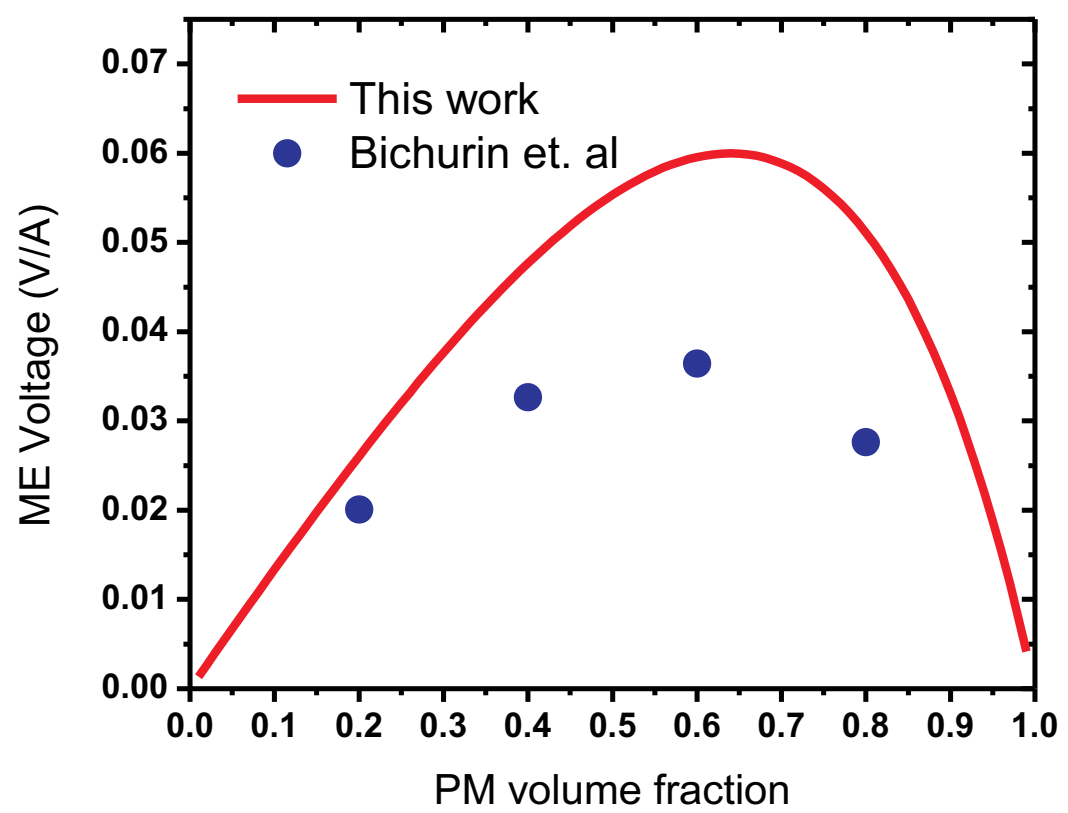

Figure 2.3: Comparison between the ME voltage coefficient obtained in this work and the work made by Bichurin et al. [49] for LSMO/PZT in TT configuration.

$E^{a v g}=0$, resulting in the following formula

$$
\begin{aligned}
\beta_{T T}= & \frac{C_{1}}{D_{1}} \\
C_{1}= & \left(2 d_{31}^{P E} \epsilon_{33}^{P M} \mu_{33}^{P E} q_{31}^{P M}(\chi-1) \chi\right) \\
D_{1}= & \left(-\epsilon_{33}^{P M}(\chi-1)\left(2\left(q_{31}^{P M}\right)^{2}(\chi-1)^{2}-\left(\mu_{33}^{P M}(\chi-1)-\mu_{33}^{P E} \chi\right)\left(S_{11}^{P M}(\chi-1)+S_{12}^{P M}(\chi-1)\right.\right.\right. \\
& \left.\left.-\left(S_{11}^{P E}+S_{12}^{P E}\right) \chi\right)\right)+\chi\left(2\left(d_{31}^{P E}\right)^{2} \chi\left(\mu_{33}^{P M}+\mu_{33}^{P E} \chi-\mu_{33}^{P M} \chi\right)+\epsilon_{33}^{P E}\left(2\left(q_{31}^{P M}\right)^{2}(\chi-1)^{2}\right.\right. \\
& \left.\left.\left.-\left(\mu_{33}^{P M}(\chi-1)-\mu_{33}^{P E} \chi\right)\left(S_{11}^{P M}(\chi-1)+S_{12}^{P M}(\chi-1)-\left(S_{11}^{P E}+S_{12}^{P E}\right) \chi\right)\right)\right)\right)
\end{aligned}
$$

The values obtained with this equation were compared with those obtained using Equation 16 in [48]. The results are shown in Figure 2.4. There is good agreement between the model proposed in [48] and the present work.

To calculate the ME charge coefficient, the electric field between electrodes is set to be zero as a boundary condition. If the total electric field is zero, it does not imply that the 


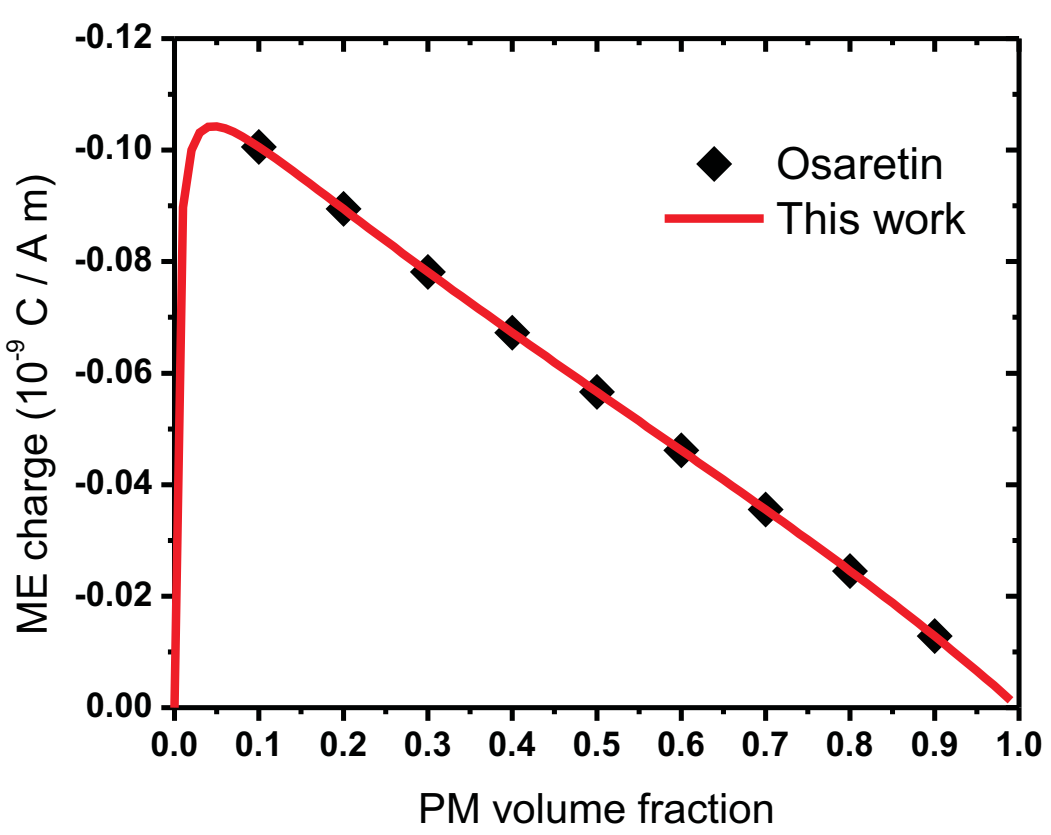

Figure 2.4: ME charge coefficient for different PM volume fractions of laminated CFO-PZT (TT) composite. The obtained results were compared with the work made by Osaretin [48].

electric field in the PM phase and the PE phase are zero, as it can be seen in (2.21), but if the total electric field and the electric field in the PM phase are zero, the electric field in the PE phase will also be zero. In the proposed model, the electric field generated in the PM phase $\left(E_{z}^{P M}\right)$ is considerably high. In real applications, the PM phase cannot produce an electric field after a period of time because the electric conductivity of this material is high (the conductivity of $\mathrm{CFO}$ is $0.01 \mathrm{Scm}^{-1}$ ) [67]. If the conductivity of the composite were to be modeled explicitly, the complexity of the model would increase significantly.

There are two ways to overcome this. First, the electric field in the PM phase can be set to be zero as an additional constraint condition $\left(E_{z}^{P M}=0\right)$. Second, the dielectric constant of the PM phase can be changed to avoid the generation of a electric field through the PM phase. As it can be seen in (2.13), in order to decrease the electric field generated in the PM phase, the dielectric constant of the PM phase, $\epsilon_{33}^{P M}$, has to be increased. Both methods work equally well within the context of the model proposed herein but we wish to emphasize 


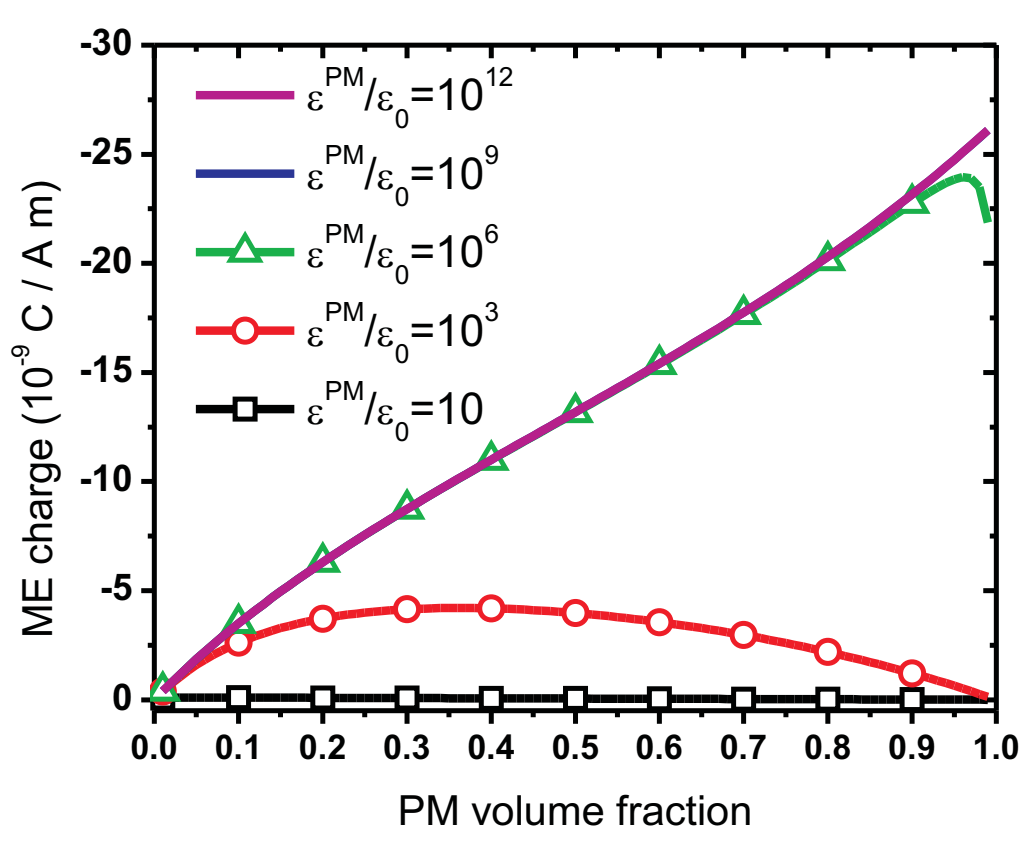

Figure 2.5: ME charge coefficient for different dielectric constants of the PM phase of the laminated CFO-PZT (TT) composite.

the fact that, unless the electric field in the PM phase is explicitly set to zero, the dielectric constant of the PM phase must be increased to a high value. Otherwise, model results will not be accurate, and sometimes this fact has been overlooked in the literature.

The ME charge coefficients for different values of the dielectric constant of the PM phase are shown in Figure 2.5. The higher the dielectric constant of the PM phase, the higher the ME charge coefficient. This trend is observed until the dielectric constant reaches values of $\epsilon_{33}^{P M} / \epsilon_{0}=10^{9}$. Higher values have no significant effect.

\subsubsection{Longitudinal magnetization and transverse polarization (LT)}

The ME charge coefficient has been measured by several researchers. The results of the proposed model are compared with experimental data reported in [68], where two layers of FeBSiC alloy were bonded to a (100) oriented PZN-PT layer, with 33\% PM volume fraction in an LT configuration. The experimental value of ME charge coefficient reported is $168 \times$ 
$10^{-9} \mathrm{CA}^{-1} \mathrm{~m}^{-1}$. To compare this values with the current model, the analytical expressions for the LT configuration are obtained as follows.

To obtain the ME coefficients for the LT configuration, a similar approach to the TT configuration is used. Using (2.1) for the first and second terms of the strain vector in the PE phase, the following equations are obtained

$$
\begin{aligned}
& s_{x}^{P E}=S_{11}^{P E} \sigma_{x}^{P E}+S_{12}^{P E} \sigma_{y}^{P E}+d_{31}^{P E} E_{z}^{P E} \\
& s_{y}^{P E}=S_{12}^{P E} \sigma_{x}^{P E}+S_{11}^{P E} \sigma_{y}^{P E}+d_{31}^{P E} E_{z}^{P E}
\end{aligned}
$$

In this case $\sigma_{x} \neq \sigma_{y}$ since the magnetic field applied in the y-direction will produce different strains in the $\mathrm{x}$ and $\mathrm{y}$-direction. In the case of the PM phase, the first and second terms of the strain vector are

$$
\begin{aligned}
& s_{x}^{P M}=S_{11}^{P M} \sigma_{x}^{P M}+S_{23}^{P M} \sigma_{y}^{P M}+q_{31}^{P M} H_{y}^{P M} \\
& s_{y}^{P M}=S_{23}^{P M} \sigma_{x}^{P M}+S_{33}^{P M} \sigma_{y}^{P M}+q_{33}^{P M} H_{y}^{P M}
\end{aligned}
$$

From (2.2), the electric displacement in the z-direction for the PE phase is expressed as

$$
D_{z}^{P E}=d_{31}^{P E} \sigma_{x}^{P E}+d_{31}^{P E} \sigma_{y}^{P E}+\epsilon_{33}^{P E} E_{z}^{P E}
$$

and the following equation is obtained for the PM phase

$$
D_{z}^{P M}=\epsilon_{11}^{P M} E_{z}^{P M}
$$

The same procedure is used to obtain the magnetic flux density in the y-direction for the PM phase

$$
B_{y}^{P M}=q_{31}^{P M} \sigma_{x}^{P M}+q_{33}^{P M} \sigma_{y}^{P M}+\mu_{33}^{P M} H_{y}^{P M}
$$


and for the PE phase

$$
B_{y}^{P E}=\mu_{11}^{P E} H_{y}^{P E}
$$

The strain in the $\mathrm{x}$ and $\mathrm{y}$-direction are the same for the PM and PE layers, assuming they are perfectly bonded

$$
\begin{aligned}
& s_{x}^{P E}=s_{x}^{P M} \\
& s_{y}^{P E}=s_{y}^{P M}
\end{aligned}
$$

By force equilibrium, the force produced be the PM phase restricted by the PE phase has the same magnitude and opposite directions

$$
\begin{aligned}
\sigma_{x}^{P M} & =-\sigma_{x}^{P E}\left(\frac{1}{\chi}-1\right) \\
\sigma_{y}^{P M} & =-\sigma_{y}^{P E}\left(\frac{1}{\chi}-1\right)
\end{aligned}
$$

The electric displacement is the same in the PE and the PM phases because the charge is produced between the top and the bottom of the laminate

$$
D_{z}^{P M}=D_{z}^{P E}
$$

Since the magnetic field is applied in the y-direction, the magnetic field in each phase has to be equal

$$
H_{y}^{P M}=H_{y}^{P E}
$$

Again, it is convenient to define the averaged electric field, since this is the value which 
is externally measured:

$$
E^{a v g}=E_{z}^{P E}(1-\chi)+E_{z}^{P M} \chi
$$

The ME voltage and charge coefficient is obtained by solving the system of equations (2.27)-(2.41) and setting the electric displacement equal to zero $\left(D_{z}=0\right)$

$$
\begin{aligned}
\alpha_{L T}= & \frac{A_{2}}{B_{2}} \\
A_{2}= & d_{31}^{P E}(\chi-1) \chi\left(q_{33}^{P M}\left(\left(S_{23}^{P M}-S_{11}^{P M}\right)(1-\chi)-\left(S_{11}^{P E}-S_{12}^{P E}\right)\right) \chi\right) \\
& +q_{31}^{P M}\left(S_{23}^{P M}(1-\chi)+S_{33}^{P M}(\chi-1)+\left(-S_{11}^{P E}+S_{12}^{P E}\right) \chi\right) \\
B_{2}= & \left(d_{31}^{P E}\right)^{2} \chi\left(S_{11}^{P M}-2 S_{23}^{P M}(1-\chi)+S_{33}^{P M}(1-\chi)-\left(-2 S_{11}^{P E}+2 S_{12}^{P E}+S_{11}^{P M}\right) \chi\right) \\
& \epsilon_{33}^{P E}\left(\left(S_{23}^{P M}\right)^{2}+\left(\left(S_{12}^{P E}-S_{23}^{P M}\right)^{2}-\left(S_{11}^{P E}-S_{11}^{P M}\right)\left(S_{11}^{P E}-S_{33}^{P M}\right)\right) \chi^{2}\right) \\
& -S_{11}^{P M} S_{33}^{P M}-\left(2 S_{23}^{P M}\left(-S_{12}^{P E}+S_{23}^{P M}\right)-2 S_{11}^{P M} S_{33}^{P M}+S_{11}^{P E}\left(S_{11}^{P M}+S_{33}^{P M}\right)\right) \chi
\end{aligned}
$$

The ME charge coefficient is obtained by solving the system of equations (2.27)-(2.41) and the average electric field equal to zero $\left(E^{a v g}=0\right)$

$$
\begin{aligned}
\beta_{L T}= & \frac{d_{31}^{P E}(\chi-1) \chi\left(C_{2}\right)}{(1-\chi)\left(D_{2}\right)+\frac{\chi}{\epsilon_{11}^{P M}}\left(E_{2}+\epsilon_{33}^{P E}\left(\left(S_{23}^{P M}\right)^{2}-S_{11}^{P M} S_{33}^{P M}-F_{2}+G_{2}\right)\right)} \\
C_{2}= & q_{33}^{P M}\left(S_{11}^{P M}-S_{23}^{P M}+\left(S_{11}^{P E}-S_{12}^{P E}-S_{11}^{P M}+S_{23}^{P M}\right) \chi\right) \\
& q_{31}^{P M}\left(S_{33}^{P M}+S_{23}^{P M}(\chi-1)-\left(-S_{11}^{P E}+S_{12}^{P E}+S_{33}^{P M}\right) \chi\right) \\
D_{2}= & \left(S_{23}^{P M}\right)^{2}-S_{11}^{P M} S_{33}^{P M}+\left(\left(S_{12}^{P E}-S_{23}^{P M}\right)^{2}-\left(S_{11}^{P E}-S_{11}^{P M}\right)\left(S_{11}^{P E}-S_{33}^{P M}\right)\right) \chi^{2} \\
& -\left(2 S_{23}^{P M}\left(-S_{12}^{P E}+S_{23}^{P M}\right)-2 S_{11}^{P M} S_{33}^{P M}+S_{11}^{P E}\left(S_{11}^{P M}+S_{33}^{P M}\right)\right) \chi \\
E_{2}= & \left(d_{31}^{P E}\right)^{2} \chi\left(S_{11}^{P M}-2 S_{23}^{P M}+S_{33}^{P M}-\left(-2 S_{11}^{P E}+2 S_{12}^{P E}+S_{11}^{P M}-2 S_{23}^{P M}+S_{33}^{P M}\right) \chi\right) \\
F_{2}= & \left(2 S_{23}^{P M}\left(-S_{12}^{P E}+S_{23}^{P M}\right)-2 S_{11}^{P M} S_{33}^{P M}+S_{11}^{P E}\left(S_{11}^{P M}+S_{33}^{P M}\right)\right) \chi \\
G_{2}= & \left(\left(S_{12}^{P E}-S_{23}^{P M}\right)^{2}-\left(S_{11}^{P E}-S_{11}^{P M}\right)\left(S_{11}^{P E}-S_{33}^{P M}\right)\right) \chi^{2}
\end{aligned}
$$

The results obtained by this model are compared with the experimental work reported 


\begin{tabular}{c||c|c||c} 
Material Property & FeBSiC & PZN-PT & Units \\
\hline \hline$S_{11}$ & 125 & 69 & $10^{-12} m^{2} N^{-1}$ \\
\hline$S_{33}$ & 40 & 119 & $10^{-12} m^{2} N^{-1}$ \\
\hline$S_{12}$ & $-42^{a}$ & $-23^{a}$ & $10^{-12} m^{2} N^{-1}$ \\
\hline$S_{23}$ & $-13^{a}$ & & $10^{-12} m^{2} N^{-1}$ \\
\hline$\epsilon / \epsilon_{0}$ & see text & 5500 & \\
\hline$\mu / \mu_{0}$ & 2 & 1 & \\
\hline$d_{31}$ & 0 & -2800 & $10^{-12} C N^{-1}$ \\
\hline$q_{31}$ & -5800 & 0 & $10^{-12} m A^{-1}$ \\
\hline$q_{33}$ & 12000 & 0 & $10^{-12} m A^{-1}$ \\
\hline
\end{tabular}

Table 2.2: Material Properties of PZN-PT and FeBSiC from [68]. ${ }^{a}$ were calculated by assuming $\nu_{12}^{P E}=\nu_{12}^{P M}=\nu_{23}^{P M}=1 / 3$

in [68]. The materials used for the PM and PE phase are FeBSiC and (001) PZN-PT, with properties listed in Table 2.2. Values for $S_{12}^{P E}, S_{12}^{P M}$ and $S_{23}^{P M}$ were calculated by assuming $\nu_{12}^{P E}=\nu_{12}^{P M}=\nu_{31}^{P M}=1 / 3$, where $\nu$ is the Poisson ratio $\left(S_{12}=-\nu_{12} S_{11}\right.$ and $\left.S_{23}^{P M}=-\nu_{31}^{P M} S_{33}^{P M}\right)$.

The ME charge coefficient reported in [68] and the predictions obtained using different values for the relative dielectric permittivity are shown in Figure 2.6. When $\epsilon^{P M} / \epsilon_{0}=10$ is used, the calculated ME charge coefficient $(\chi=0.33)$ is $7.1 \times 10^{-9} \mathrm{CA}^{-1} \mathrm{~m}^{-1}$, which is quite smaller than the experimentally measured value $\left(168 \times 10^{-9} \mathrm{C}^{-1} \mathrm{~m}^{-1}\right)$. As previously discussed, this is due to the electric field produced in the PM phase. When the ME charge coefficient is calculated using higher values for the relative dielectric permittivity of the PM phase, $\beta$ increases and the electric field in the PM phase decreases. It can be seen that the values converge to a single line. No difference is observed for $\epsilon^{P M} / \epsilon_{0}>10^{6}$, and the electric field in the PM phase $E \rightarrow 0$. This indicates that using a high relative dielectric permittivity is a valid approach to to take into account the high conductivity of the PM phase. Furthermore, the calculated ME charge coefficient $\left(201 \times 10^{-9} \mathrm{CA}^{-1} \mathrm{~m}^{-1}\right)$ when the electric field in the PM phase approaches zero, is in reasonable agreement with experimental data reported in [68] $\left(168 \times 10^{-9} \mathrm{CA}^{-1} \mathrm{~m}^{-1}\right)$.

The results obtained when the electric field in the PM phase is zero show that the ME charge coefficient increases when the PM volume fraction increases. This is due to the fact 


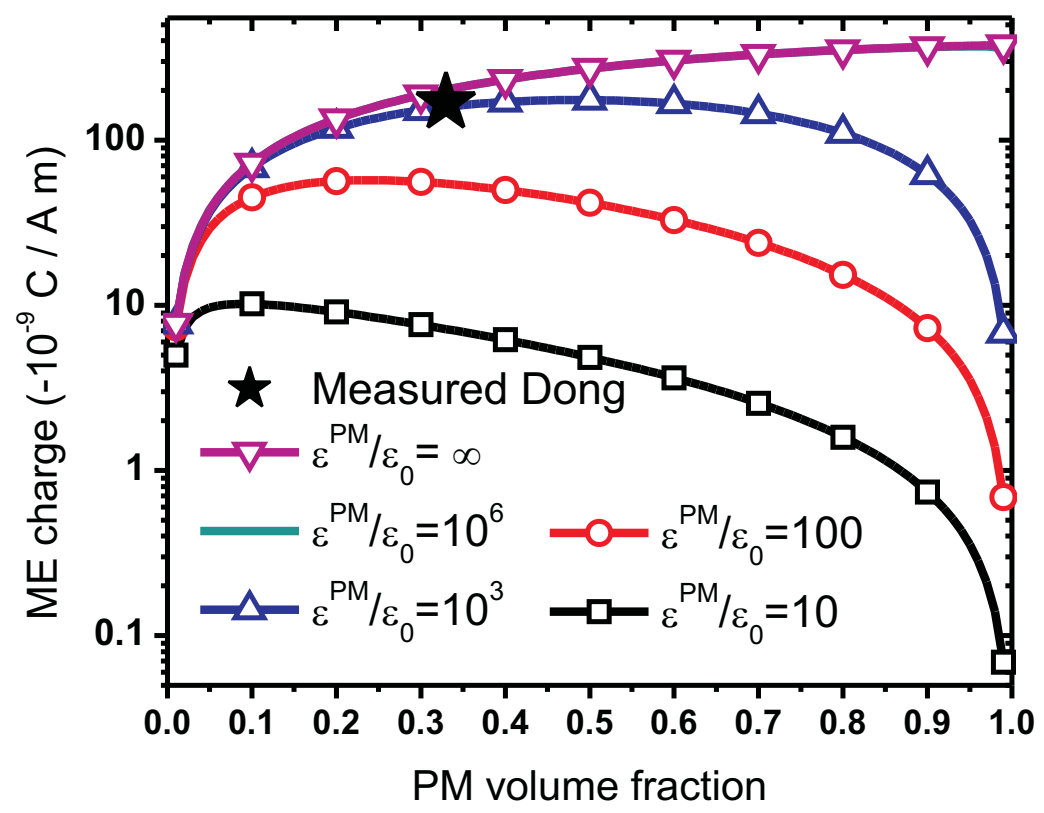

Figure 2.6: ME charge coefficient (LT) of the laminated FeBSiC-PZN-PT composite for different PM relative dielectric permittivities compared with the experimental result obtained by Dong et al. [68]. 
that higher amounts of PM phase will produce higher strain in the PE phase, resulting in a higher electric displacement in the PE phase. Since the electric displacement, $D$, does not depend on the PE phase thickness no decrease in the ME charge coefficient can be seen when higher amounts of the PM phase are used.

Using $\epsilon^{P M} \rightarrow \infty$, the expressions for the ME charge coefficient, in TT and LT configurations, reduce to

$$
\begin{aligned}
\beta_{T T}= & \frac{\left(2 d_{31}^{P E} \mu_{33}^{P E} q_{31}^{P M}(\chi-1) \chi\right)}{E_{1}} \\
E_{1}= & (1-\chi)\left(2\left(q_{31}^{P M}\right)^{2}(\chi-1)^{2}-\left(\mu_{33}^{P M}(\chi-1)-\mu_{33}^{P E} \chi\right)\right. \\
& \left.\left(S_{11}^{P M}(\chi-1)+S_{12}^{P M}(\chi-1)-\left(S_{11}^{P E}+S_{12}^{P E}\right) \chi\right)\right) \\
\beta_{L T}= & \frac{d_{31}^{P E}(\chi-1) \chi\left(C_{3}\right)}{(1-\chi)\left(D_{3}\right)}
\end{aligned}
$$

\subsubsection{TL configuration}

A similar approach is used to obtain the ME charge and voltage coefficient for the TL configuration, as follows

$$
\begin{aligned}
\alpha_{T L}= & \frac{A_{3}}{B_{3}} \\
A_{3}= & -\left(\mu _ { 1 1 } ^ { P E } q _ { 3 1 } ^ { P M } \chi \left(-\left(d_{31}^{P E}+d_{33}^{P E}\right)\left(S_{11}^{P M}-S_{12}^{P M}\right)+\left(d _ { 3 3 } ^ { P E } \left(-S_{11}^{P E}+S_{23}^{P E}+S_{11}^{P M}\right.\right.\right.\right. \\
& \left.\left.\left.\left.-S_{12}^{P M}\right)+d_{31}^{P E}\left(S_{23}^{P E}-S_{33}^{P E}+S_{11}^{P M}-S_{12}^{P M}\right)\right) \chi\right)\right) \\
B_{3}= & \epsilon_{33}^{P E}\left(( q _ { 3 1 } ^ { P M } ) ^ { 2 } ( \chi - 1 ) ^ { 2 } \left(-2 S_{11}^{P M}(\chi-1)+2 S_{12}^{P M}(\chi-1)+\left(S_{11}^{P E}-2 S_{23}^{P E}\right.\right.\right. \\
& \left.\left.+S_{33}^{P E}\right) \chi\right)+\left(\mu_{33}^{P M}(\chi-1)-\mu_{11}^{P E} \chi\right)\left(\left(S_{11}^{P M}\right)^{2}(\chi-1)^{2}-\left(S_{12}^{P M}\right)^{2}(\chi-1)^{2}\right. \\
& \left.\left.-\left(S_{11}^{P E}+S_{33}^{P E}\right) S_{11}^{P M}(\chi-1) \chi+2 S_{23}^{P E} S_{12}^{P M}(\chi-1) \chi+\left(-\left(S_{23}^{P E}\right)^{2}+S_{11}^{P E} S_{33}^{P E}\right) \chi^{2}\right)\right) \\
& +\chi\left(\left(d_{33}^{P E}\right)^{2}\left(-\left(q_{31}^{P M}\right)^{2}(\chi-1)^{2}+\left(\mu_{33}^{P M}(\chi-1)-\mu_{11}^{P E} \chi\right)\left(S_{11}^{P M}(\chi-1)-S_{11}^{P E} \chi\right)\right)\right. \\
& +2 d_{31}^{P E} d_{33}^{P E}\left(\left(q_{31}^{P M}\right)^{2}(\chi-1)^{2}-\left(\mu_{33}^{P M}(\chi-1)-\mu_{11}^{P E} \chi\right)\left(S_{12}^{P M}(\chi-1)-S_{23}^{P E} \chi\right)\right) \\
& \left.+\left(d_{31}^{P E}\right)^{2}\left(-\left(q_{31}^{P M}\right)^{2}(\chi-1)^{2}+\left(\mu_{33}^{P M}(\chi-1)-\mu_{11}^{P E} \chi\right)\left(S_{11}^{P M}(\chi-1)-S_{33}^{P E} \chi\right)\right)\right)
\end{aligned}
$$


and

$$
\begin{aligned}
\beta_{T L}= & \frac{C_{3}}{D_{3}} \\
C_{3}= & -\left(\mu _ { 1 1 } ^ { P E } q _ { 3 1 } ^ { P M } ( \chi - 1 ) \chi \left(-\left(d_{31}^{P E}+d_{33}^{P E}\right)\left(S_{11}^{P M}-S_{12}^{P M}\right)+\left(d_{33}^{P E}\right.\right.\right. \\
& \left.\left.\left.\left(-S_{11}^{P E}+S_{23}^{P E}+S_{11}^{P M}-S_{12}^{P M}\right)+d_{31}^{P E}\left(S_{23}^{P E}-S_{33}^{P E}+S_{11}^{P M}-S_{12}^{P M}\right)\right) \chi\right)\right) \\
D_{3}= & \left(q_{31}^{P M}\right)^{2}(\chi-1)^{2}\left(-2 S_{11}^{P M}(\chi-1)+2 S_{12}^{P M}(\chi-1)+\left(S_{11}^{P E}-2 S_{23}^{P E}+S_{33}^{P E}\right) \chi\right) \\
& +\left(\mu_{33}^{P M}(\chi-1)-\mu_{11}^{P E} \chi\right)\left(\left(S_{11}^{P M}\right)^{2}(\chi-1)^{2}-\left(S_{12}^{P M}\right)^{2}(\chi-1)^{2}\right. \\
& \left.-\left(S_{11}^{P E}+S_{33}^{P E}\right) S_{11}^{P M}(\chi-1) \chi+2 S_{23}^{P E} S_{12}^{P M}(\chi-1) \chi+\left(-\left(S_{23}^{P E}\right)^{2}+S_{11}^{P E} S_{33}^{P E}\right) \chi^{2}\right)
\end{aligned}
$$

\subsubsection{LL configuration}

A similar approach is used to obtain the ME charge and voltage coefficient for the LL configuration, obtaining

$$
\begin{aligned}
\alpha_{L L}= & \frac{A_{4}}{B_{4}} \\
A_{4}= & \chi\left(d_{33}^{P E}\left(q_{33}^{P M}\left(S_{11}^{P M}(\chi-1)-S_{11}^{P E} \chi\right)+q_{31}^{P M}\left(S_{23}^{P M}+S_{23}^{P E} \chi-S_{23}^{P M} \chi\right)\right)\right. \\
& \left.+d_{31}^{P E}\left(q_{31}^{P M}\left(S_{33}^{P M}(\chi-1)-S_{33}^{P E} \chi\right)+q_{33}^{P M}\left(S_{23}^{P M}+S_{23}^{P E} \chi-S_{23}^{P M} \chi\right)\right)\right) \\
B_{4}= & \chi\left(\left(d_{33}^{P E}\right)^{2}\left(S_{11}^{P M}(\chi-1)-S_{11}^{P E} \chi\right)+\left(d_{31}^{P E}\right)^{2}\left(S_{33}^{P M}(\chi-1)-S_{33}^{P E} \chi\right)\right. \\
& \left.+2 d_{31}^{P E} d_{33}^{P E}\left(S_{23}^{P M}+S_{23}^{P E} \chi-S_{23}^{P M} \chi\right)\right)+\epsilon_{33}^{P E}\left(-\left(S_{23}^{P M}\right)^{2}(\chi-1)^{2}\right. \\
& +2 S_{23}^{P E} S_{23}^{P M}(\chi-1) \chi+S_{11}^{P M}\left(S_{33}^{P M}+S_{33}^{P E} \chi\right)+\chi\left(\left(S_{11}^{P E}\right.\right. \\
& \left.\left.\left.-2 S_{11}^{P M}\right) S_{33}^{P M}-\left(S_{23}^{P E}\right)^{2} \chi+\left(S_{11}^{P E}-S_{11}^{P M}\right)\left(S_{33}^{P E}-S_{33}^{P M}\right) \chi\right)\right)
\end{aligned}
$$


and

$$
\begin{aligned}
\beta_{L L}= & \frac{C_{4}}{D_{4}} \\
C_{4}= & (\chi-1) \chi\left(d_{33}^{P E}\left(q_{33}^{P M}\left(S_{11}^{P M}(\chi-1)-S_{11}^{P E} \chi\right)+q_{31}^{P M}\left(S_{23}^{P M}+S_{23}^{P E} \chi-S_{23}^{P M} \chi\right)\right)\right. \\
& \left.+d_{31}^{P E}\left(q_{31}^{P M}\left(S_{33}^{P M}(\chi-1)-S_{33}^{P E} \chi\right)+q_{33}^{P M}\left(S_{23}^{P M}+S_{23}^{P E} \chi-S_{23}^{P M} \chi\right)\right)\right) \\
D_{4}= & -\left(S_{23}^{P M}\right)^{2}(\chi-1)^{2}+2 S_{23}^{P E} S_{23}^{P M}(\chi-1) \chi+S_{11}^{P M}\left(S_{33}^{P M}+S_{33}^{P E} \chi\right) \\
& +\chi\left(\left(S_{11}^{P E}-2 S_{11}^{P M}\right) S_{33}^{P M}-\left(S_{23}^{P E}\right)^{2} \chi+\left(S_{11}^{P E}-S_{11}^{P M}\right)\left(S_{33}^{P E}-S_{33}^{P M}\right) \chi\right)
\end{aligned}
$$

\subsection{ME coupling Factor}

The ME coupling factor is the ratio between the electrical work generated and magnetic work applied (or magnetic work generated and electrical work applied). It is an useful property to compare different ME composites in their ability to be used as energy transducers. The mathematical definition is the following

$$
\kappa^{2}=\frac{W_{E}^{G}}{W_{M}^{A}}
$$

where $W_{E}^{G}$ is the electric work generated, $W_{M}^{A}$ is the magnetic work applied, $W_{M}^{G}$ is the magnetic work generated, and $W_{E}^{A}$ is the electric work applied. The ME coupling factor can be expressed as a function of the ME charge coefficient and the electric and magnetic properties of the composite.

If a magnetic field is applied to the composite when it is short circuited $\left(E_{3}^{I->I I}=0\right)$, no electric work will be generated and magnetic work will be applied with the following magnitude (state I to state II in Figure 2.7)

$$
\left.\left.W_{M}^{A}=\frac{B_{z}^{I I} \times H_{z}^{I I}}{2}\right]_{E=0}=\frac{\mu_{z z}\left(H_{z}^{I I}\right)^{2}}{2}\right]_{E=0}=\frac{\mu_{z z}^{E}\left(D_{z}^{I I}\right)^{2}}{2\left(\beta_{z z}\right)^{2}}
$$

where $\mu_{z z}^{E}$ is the magnetic permeability in the z-direction of the composite measured at con- 

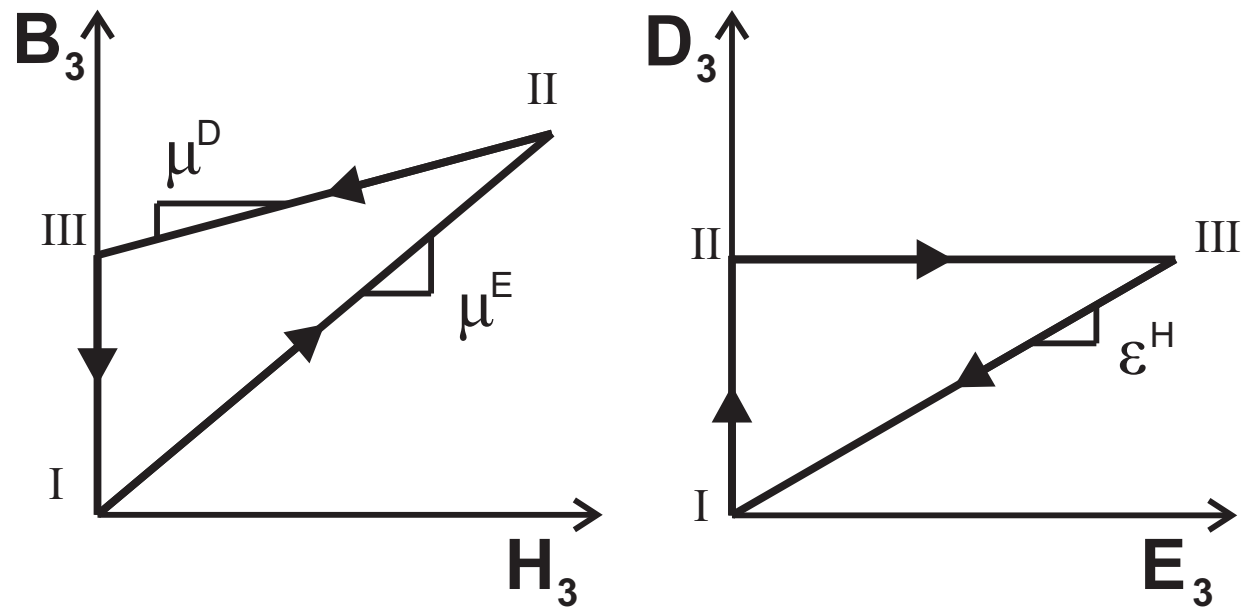

Figure 2.7: B-H and D-E diagram used to calculate the magnetic and electric work defined in ME coupling factor.

stant electric field and $\left.\beta=\frac{D}{H}\right]_{E=0}$. Prior to the removal of the magnetic field the composite is open-circuited. From the state II to the state III, there is no electric work generated and the generated magnetic work is wasted. After this it is connected to an ideal electric load to complete the cycle. From the state III to the state I only electric work is generated. The amount of work generated is

$$
\begin{aligned}
W_{E}^{G} & \left.=\frac{D_{z}^{I I I} \times E_{z}^{I I I}}{2}\right]_{H=0} \\
& \left.=\frac{\left(D_{z}^{I I I}\right)^{2}}{2 \epsilon_{z z}}\right]_{H=0}=\frac{\left(D_{z}^{I I I}\right)^{2}}{2 \epsilon_{z z}^{H}}=\frac{\left(D_{z}^{I I}\right)^{2}}{2 \epsilon_{z z}^{H}}
\end{aligned}
$$

where $\epsilon_{z z}^{H}$ is the dielectric permittivity in the z-direction of the composite measured at constant magnetic field. With these values the ME coupling factor can be calculated as

$$
\begin{aligned}
\kappa_{z z}^{2} & =\frac{\left(D_{z}^{I I}\right)^{2}}{2 \epsilon_{z z}^{H}} \frac{2\left(\beta_{z z}\right)^{2}}{\mu_{z z}^{E}\left(D_{z}^{I I}\right)^{2}}=\frac{\left(\beta_{z z}\right)^{2}}{\epsilon_{z z}^{H} \mu_{z z}^{E}} \\
& =\frac{\left(\alpha_{z z}\right)^{2} \epsilon_{z z}^{H}}{\mu_{z z}^{E}}
\end{aligned}
$$

taking into account that $\beta_{i j}=\frac{\alpha_{i j}}{\epsilon_{j j}^{H}}$. The reciprocal of the ME coupling factor can be calculated 
as

$$
\kappa^{2}=\frac{W_{M}^{G}}{W_{E}^{A}}=\frac{W_{E}^{G}}{W_{M}^{A}}
$$

and can be seen that these two definitions are equivalent.

Similarly, $\kappa_{i j}$ is obtained as

$$
\kappa_{i j}^{2}=\frac{\left(\beta_{i j}\right)^{2}}{\epsilon_{j j}^{H} \mu_{i i}^{E}}=\frac{\left(\alpha_{i j}\right)^{2} \epsilon_{j j}^{H}}{\mu_{i i}^{E}}
$$

Where the subscripts $i$ and $j$ correspond to the polarization and magnetization selected (Transverse or Longitudinal). The dielectric permittivity at constant magnetic field and magnetic permeability at constant electric displacement of the composite has to be calculated for all the different configurations. To do this the simple formulas for parallel and series capacitors cannot be used because in this case, fixing the magnetic field has an influence on the dielectric permittivity. It can be seen in (2.2) that for the composite, whenever an electric field is applied to measure the dielectric permittivity, a strain is produced that results in the increase of the magnetic flux density or magnetic field. Therefore, to calculate the dielectric permittivity of the composite, $\epsilon^{H}$, a similar approach as the one used for the calculation of the ME voltage coefficient $\alpha$ must be used.

To calculate $\alpha$, the ratio between the electric field and the magnetic field at a constant electric displacement was evaluated. In the case of $\epsilon^{H}$, the ratio between the electric displacement and the electric field at constant magnetic field has to be calculated as follows

$$
\left.\epsilon_{i j}^{H}=\frac{D_{i}}{E_{j}}\right]_{H=0}
$$

Similarly the magnetic permeability is calculated

$$
\left.\mu_{i j}^{D}=\frac{B_{i}}{H_{j}}\right]_{D=0}
$$


For the TT configuration the following expression are obtained

$$
\begin{aligned}
\epsilon_{z z}^{H}= & \frac{E_{1}}{F_{1}} \\
E_{1}= & -\left(\epsilon _ { 3 3 } ^ { P M } \mu _ { 3 3 } ^ { P M } ( \frac { 1 } { \chi } - 1 ) ( \mu _ { 3 3 } ^ { P M } ( \chi - 1 ) - \mu _ { 3 3 } ^ { P E } \chi ) \left(-\left(\left(2\left(d_{31}^{P E}\right)^{2} \chi\right) /(\chi-1)\right)\right.\right. \\
& +\epsilon_{33}^{P E}\left(S_{11}^{P E}+S_{12}^{P E}-S_{11}^{P M}-S_{12}^{P M}+\left(S_{11}^{P E}+S_{12}^{P E}\right) /(\chi-1)\right. \\
& \left.\left.\left.-\left(2\left(q_{31}^{P M}\right)^{2}(\chi-1)\right)\left(\mu_{33}^{P M}+\mu_{33}^{P E} \chi-\mu_{33}^{P M} \chi\right)\right)\right)\right) \\
F_{1}= & \left(\mu _ { 3 3 } ^ { P M } ( \frac { 1 } { \chi } - 1 ) ( \epsilon _ { 3 3 } ^ { P M } ( \chi - 1 ) - \epsilon _ { 3 3 } ^ { P E } \chi ) \left(\mu_{33}^{P M}\left(S_{11}^{P M}+S_{12}^{P M}\right)+2\left(q_{31}^{P M}\right)^{2}(\chi-1)\right.\right. \\
& \left.+\left(\mu_{33}^{P E}-\mu_{33}^{P M}\right)\left(S_{11}^{P M}+S_{12}^{P M}\right) \chi\right)-\mu_{33}^{P M}\left(\mu_{33}^{P M}(\chi-1)-\mu_{33}^{P E} \chi\right)\left(2\left(d_{31}^{P E}\right)^{2} \chi\right. \\
& \left.\left.+\left(S_{11}^{P E}+S_{12}^{P E}\right)\left(\epsilon_{33}^{P M}(\chi-1)-\epsilon_{33}^{P E} \chi\right)\right)\right)
\end{aligned}
$$

and

$$
\begin{aligned}
\mu_{z z}^{D}= & \frac{G_{1}}{H_{1}} \\
G_{1}= & \left(\mu _ { 3 3 } ^ { P E } \mu _ { 3 3 } ^ { P M } \left(1+\left(2 \mu _ { 3 3 } ^ { P E } ( q _ { 3 1 } ^ { P M } ) ^ { 2 } ( \frac { 1 } { \chi } - 1 ) \chi \left(\epsilon_{33}^{P M}+\epsilon_{33}^{P E} \chi\right.\right.\right.\right. \\
& \left.\left.-\epsilon_{33}^{P M} \chi\right)\right) /\left(\mu _ { 3 3 } ^ { P M } ( \frac { 1 } { \chi } - 1 ) ( \epsilon _ { 3 3 } ^ { P M } ( \chi - 1 ) - \epsilon _ { 3 3 } ^ { P E } \chi ) \left(\mu_{33}^{P M}\left(S_{11}^{P M}+S_{12}^{P M}\right)\right.\right. \\
& \left.+2\left(q_{31}^{P M}\right)^{2}(\chi-1)+\left(\mu_{33}^{P E}-\mu_{33}^{P M}\right)\left(S_{11}^{P M}+S_{12}^{P M}\right) \chi\right)-\mu_{33}^{P M}\left(\mu_{33}^{P M}(\chi-1)\right. \\
& \left.\left.\left.\left.-\mu_{33}^{P E} \chi\right)\left(2\left(d_{31}^{P E}\right)^{2} \chi+\left(S_{11}^{P E}+S_{12}^{P E}\right)\left(\epsilon_{33}^{P M}(\chi-1)-\epsilon_{33}^{P E} \chi\right)\right)\right)\right)\right) \\
H_{1}= & \left(\mu_{33}^{P M}+\mu_{33}^{P E} \chi-\mu_{33}^{P M} \chi\right)
\end{aligned}
$$

Expressions for the LT, TL and LL configurations can be obtained by following the same procedure. These expressions and all the other coefficients calculated in this work are provided as supplemental material in the Website [69]. 


\subsection{Demagnetizing Factor}

As it was previously discussed in the introduction, when a structure is exposed to an external magnetic field $\left(H_{0}\right)$ the internal magnetic field is reduced by the demagnetization field,

$$
H_{3}=H_{0}+H_{d}=H_{0}-N_{3} M
$$

where $H_{d}$ is the demagnetizing field, $N$ is the demagnetizing factor and $M$ is the magnetization. The magnetization can be written as follows,

$$
M=\frac{H_{0}\left(\mu_{r}-1\right)}{1+N_{3}\left(\mu_{r}-1\right)}
$$

resulting in,

$$
H_{3}=H_{0}\left(1-\frac{N_{3}\left(\mu_{r}-1\right)}{1+N_{3}\left(\mu_{r}-1\right)}\right)
$$

The demagnetizing factor has values between 0 and 1. When $N=0$ then $H_{3}=H_{0}$ and

when $N=1$ then $H_{3}=\frac{H_{0}}{\mu_{r}}$. The composite structures with high demagnetizing factor and high relative permeability will show the highest reduction of internal magnetic field $\left(H_{3}\right)$. This is relevant if one wishes to measure the extrinsic performance of the device, i.e., the voltage generated for a given magnetic field and specific device dimensions. However, the intrinsic material performance is independent of geometry. The extrinsic performance can be calculated combining the intrinsic material properties, as reported in this work, and the demagnetizing factor for particular device dimensions [70, 71].

The demagnetizing factor is given by the geometry of the structure, the simplest case is the sphere were $N=1 / 3$ in all three directions and it has a constant value. It has to be noticed that the demagnetization factor has to satisfy the following condition, $N_{1}+N_{2}+N_{3}=1$. In the case of an infinite plate the out of plane demagnetizing factor is 1 and the in-plane demagnetizing factor is 0 . The demagnetizing factor for non-infinite plates depends on the 
position and can be calculated using the equations derived by Joseph and Schlomann or the averaged demagnetization factor can be obtained using the expressions derived by Aharoni $[70,71]$.

In this chapter the demagnetizing effect is not considered because it is an extrinsic effect. The intrinsic magnetoelectric coefficients reported in this chapter are independent of the geometrical dimensions of the composite, thus simplifying the preliminary design and material selection criteria. Then, the dimensions of the magnetoelectric composite can be optimized taking into account the demagnetizing factor $N$ in Chapter 3. The optimal dimensions would yield $N=0$.

For the case of the composites with longitudinal magnetization ( $\$ 2.2 .2$ and $\S 2.2 .4)$, the thickness has to be considerably smaller than the in-plane dimensions to achieve $N=0$.

For composites with transverse magnetization ( $\$ 2.2 .1$ and $\S 2.2 .3)$, measured performance, rather than intrinsic performance, may be significantly reduced due to high demagnetization factor [72]. However, one could stack a number of ME laminates to build a device with high thickness-to-width ratio, $h>>w$, so that the device approximates a cylindrical rod, which has $\mathrm{N}=0$ along the axis of the rod. Each ME laminate (that is, each PM/PE pair) must be thin to reduce the shear lag effect [63].

\subsection{Results}

The expected application of the ME composite dictates which ME property needs to be optimized. For sensors, there are two different sensitivities: short circuit sensitivity and open circuit sensitivity. Higher short circuit sensitivity requires a higher ME charge coefficient $\beta$. Higher open circuit sensitivity requires a higher ME voltage coefficient $\alpha$. For energy transducers, such as magnetic harvesters, a high $\kappa$ is desired because in that case more electrical work will be generated for the same amount of magnetic work harvested.

Results are presented for PZT-5H/Terfenol-D. The properties of PZT-5H are obtained 


\begin{tabular}{c||c|c||c} 
Material Property & Terfenol-D & PZT-5H & Units \\
\hline \hline$S_{11}$ & 44 & 16.5 & $10^{-12} m^{2} N^{-1}$ \\
\hline$S_{33}$ & 38 & 20.7 & $10^{-12} m^{2} N^{-1}$ \\
\hline$S_{12}$ & -11 & -4.78 & $10^{-12} m^{2} N^{-1}$ \\
\hline$S_{23}$ & -16.5 & -8.45 & $10^{-12} m^{2} N^{-1}$ \\
\hline$\epsilon_{33} / \epsilon_{0}$ & $\infty$ & 3400 & \\
\hline$\mu_{11} / \mu_{0}$ & 8.1 & 1 & \\
\hline$\mu_{33} / \mu_{0}$ & 3 & 1 & \\
\hline$d_{31}$ & 0 & -274 & $10^{-12} C N^{-1}$ \\
\hline$d_{33}$ & 0 & 593 & $10^{-12} C N^{-1}$ \\
\hline$q_{31}$ & -4300 & 0 & $10^{-12} m A^{-1}$ \\
\hline$q_{33}$ & 8500 & 0 & $10^{-12} m A^{-1}$ \\
\hline$k_{31}$ & 0.33 & 0.39 & $10^{-12} C N^{-1}$ \\
\hline$k_{33}$ & 0.71 & 0.75 & $10^{-12} C N^{-1}$ \\
\hline
\end{tabular}

Table 2.3: Material Properties of PZT-5H and Terfernol-D from [73, 74].

from Morgan Electro Ceramics [73]. The selected elastic properties are measured at constant electric field.

It is important to use the appropriate elastic properties in the analysis. Otherwise, significant errors may occur. For example, the compliance in the polarization direction of PZT-5H is $S_{33}^{E}=20.7 \times 10^{-12} \mathrm{~m}^{2} \mathrm{~N}^{-1}$ at constant electric field while it is only $S_{33}^{D}=8.9 \times 10^{-12} \mathrm{~m}^{2} \mathrm{~N}^{-1}$ at constant electric displacement. The same applies to the dielectric permittivity, but in this case it should be measured at constant stress. The magnetic permeability $\mu^{E}$ of the PE phase is required for the calculations, but it is immaterial if it is measured at constant electric field or constant electric displacement because there is no PM coupling in the PE phase. Since the magnetic permeability of PZT is not reported in [73], a value $\mu_{P E} / \mu_{0}=1$ is used for the examples in this study.

Additionally, all the properties have to be measured with the same polarization since the properties change drastically with different polarization intensities and direction. For the PM phase, Terfenol-D is selected and the properties were obtained from [74]. The values of these properties are reported in Table 2.3.

The analytical expression for the ME coupling factor for the different configurations are evaluated with the properties from Table 2.3. The maximum value of the ME coupling factor 


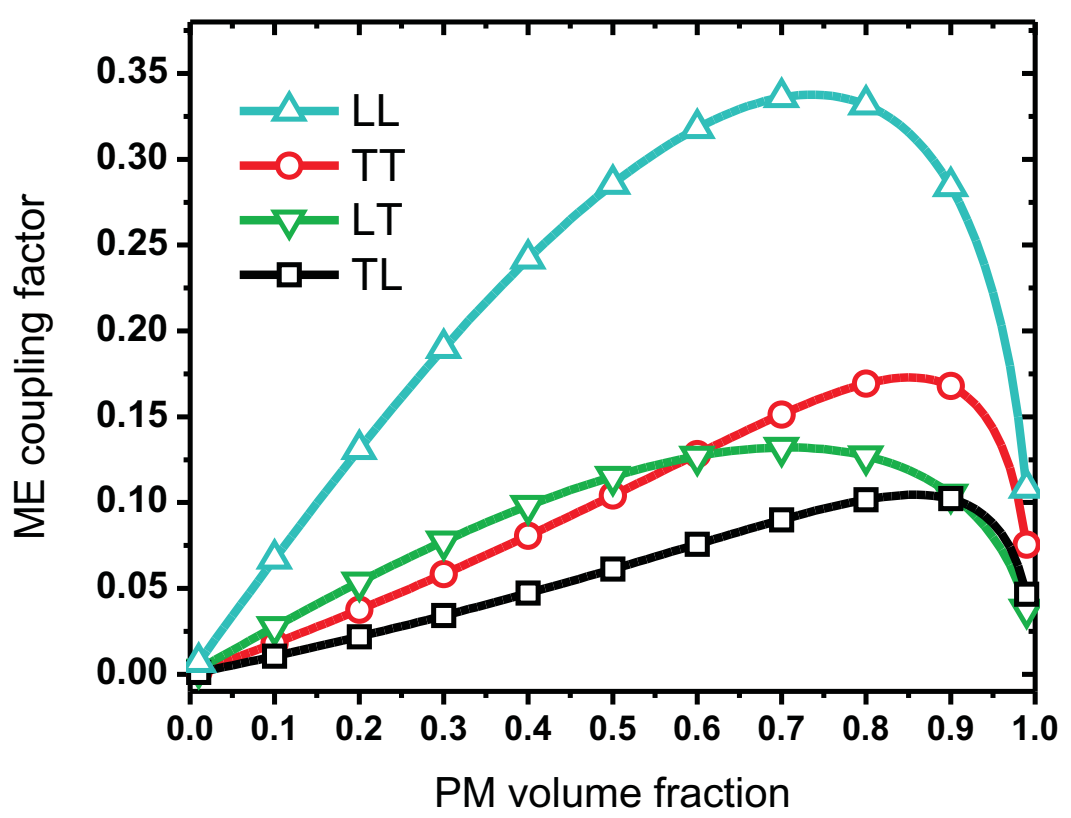

Figure 2.8: ME coupling factor of PZT-5H Terfenol-D composite for different PM volume fractions using TT, LT, TL and LL configurations.

is $\kappa=0.36$ for the LL configuration and a PM volume fraction of $73 \%$, as shown in Figure 2.8. The ME coupling factor as a function of PM volume fraction $\chi$ has a parabolic shape. This is due to the fact that for small $\chi$ the magnetic work applied cannot be transformed to mechanical work and for $\chi$ close to 1 the mechanical work generated by the PM phase cannot be converted to electrical work because the PE phase is too thin. The maximum ME coupling factor is obtained for the LL configuration because in that configuration the highest $\mathrm{PE}$ and PM coupling factors $\left(k_{33}\right)$ of the constituents are being used.

The ME charge coefficients, $\beta$, for the different configurations can be seen in Figure 2.9. For the TT and LT configuration, $\beta$ increases with increasing PM volume fraction. When higher $\chi$ is used, the stress in the PE phase increases resulting in a higher electric displacement. Taking into account that the PE area does not change, the electric displacement on the PE phase is the total electric displacement.

In the case of the LL and TL, the ME charge coefficient display a compromise between 


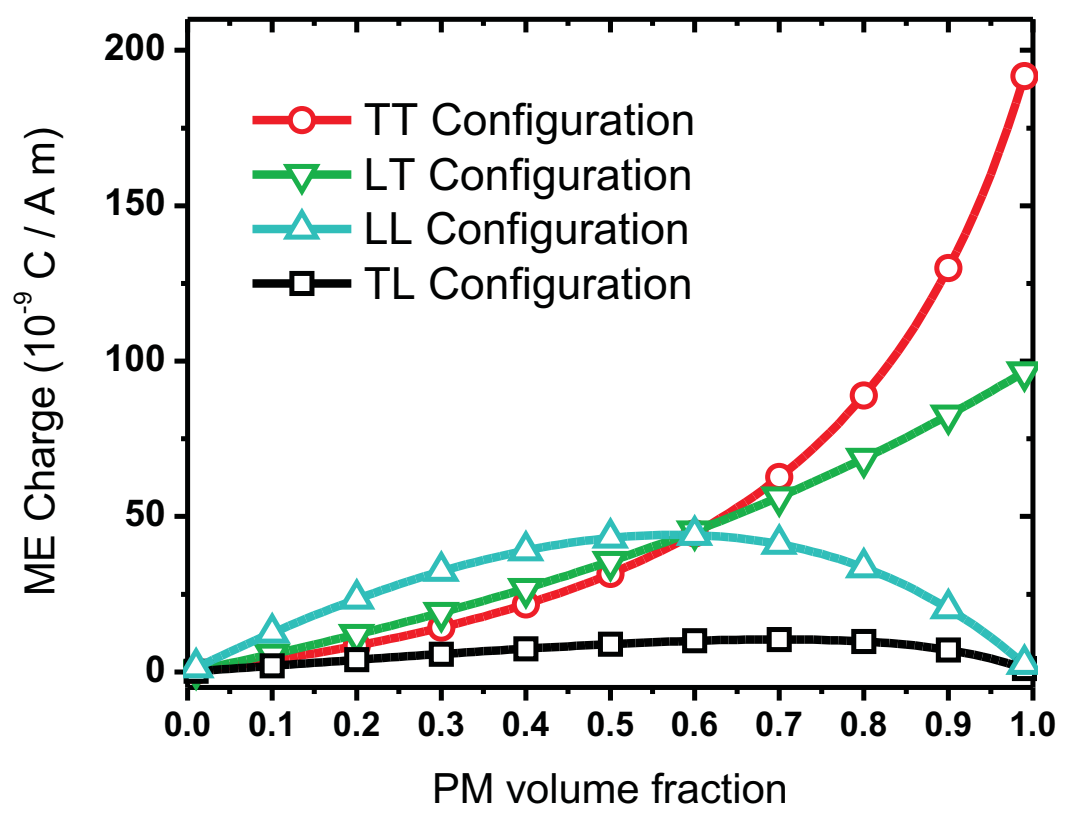

Figure 2.9: ME charge coefficient of PZT-5H Terfenol-D composite for different PM volume fractions using TT, LT, TL and LL configurations.

strain and the thickness of the PE phase. For small PM volume fraction, the increase of $\chi$ increases the strain resulting in a higher electric displacement and higher $\beta$. For high $\mathrm{PM}$ volume fraction, the increase of $\chi$ increases the electric displacement in the PE phase, but since the area of the PE phase is reduced, it results in a reduction of the total electric displacement.

The highest value of ME charge $\left(\beta_{T T}=192 \times 10^{-9} C A^{-1} m^{-1}\right)$ is obtained for the TT configuration using a PM volume fraction close to 1 . The transverse polarization has the highest charge because it has the highest effective area. In the case of magnetization, the highest charge is expected for the longitudinal magnetization (LT) because $q_{33}>q_{31}$. But this is not the case because when the magnetic field is applied, an expansion in the y-direction and a contraction in the $\mathrm{x}$-direction take place. While, the expansion in the $\mathrm{y}$-direction yields a negative electric displacement, the contraction in the x-direction yields a positive electric displacement, resulting in a small total electric displacement. Therefore, transverse 
magnetization results in higher charge because in this later case the electric displacement produced by the strain in the $\mathrm{x}$ and $\mathrm{y}$-direction are equal.

The optimum $\beta$ is obtained for volume fractions close to 1 . The use of volume fractions close to 1 will result in a small ME coupling factor. This means that if the detector used to measure charge does not have an infinite resistance, the measured charge will be diminished. For this reason, is convenient to use a lower PM volume fraction, say at 90\%, which yields a good ME charge coefficient $\left(130 \times 10^{-9} \mathrm{CA}^{-1} \mathrm{~m}^{-1}\right)$ and good ME coupling factor $(0.17)$.

The ME voltage coefficient for PZT-5H/Terfenol-D composite is shown in Figure 2.10. It shows a trend similar to that of the ME charge coefficient, but since the total electric field increases with the thickness of the PE phase, the configurations with transverse polarization (TT and LT) are the ones to show a parabolic response - not the ones with longitudinal polarization. The LL configuration shows the highest ME voltage coefficient of $\alpha_{L L}=18 \mathrm{VA}^{-1}$ with $\chi \rightarrow 1$. In the LL configuration, the electric field due to contraction/expansion in the $\mathrm{x}$ and $\mathrm{y}$-direction have the same sign because in this case the $\mathrm{PE}$ phase is polarized in the $\mathrm{y}$ direction. A PM volume fraction of $85 \%$ is more convenient to combine a high $\alpha=10.7 \mathrm{VA}^{-1}$ with a very high ME coupling factor of 0.32 .

\subsection{Conclusions}

A new method is proposed to take into account the high conductivity of the PM phase. The method consists of artificially increasing the electrical permittivity of the PM phase. From the analysis of three composites made with three PM and three PE materials, analyzed for PM volume fraction continuously varying from zero to one, we conclude that setting the relative permittivity of the PM to $10^{9}$ is equivalent to a fully conducting PM material and thus a negligible electric field in the PM.

Artificially increasing the dielectric permittivity of the PM phase in the numerical calculation to simulate the high conductivity of said phase proved to be an expedient method 


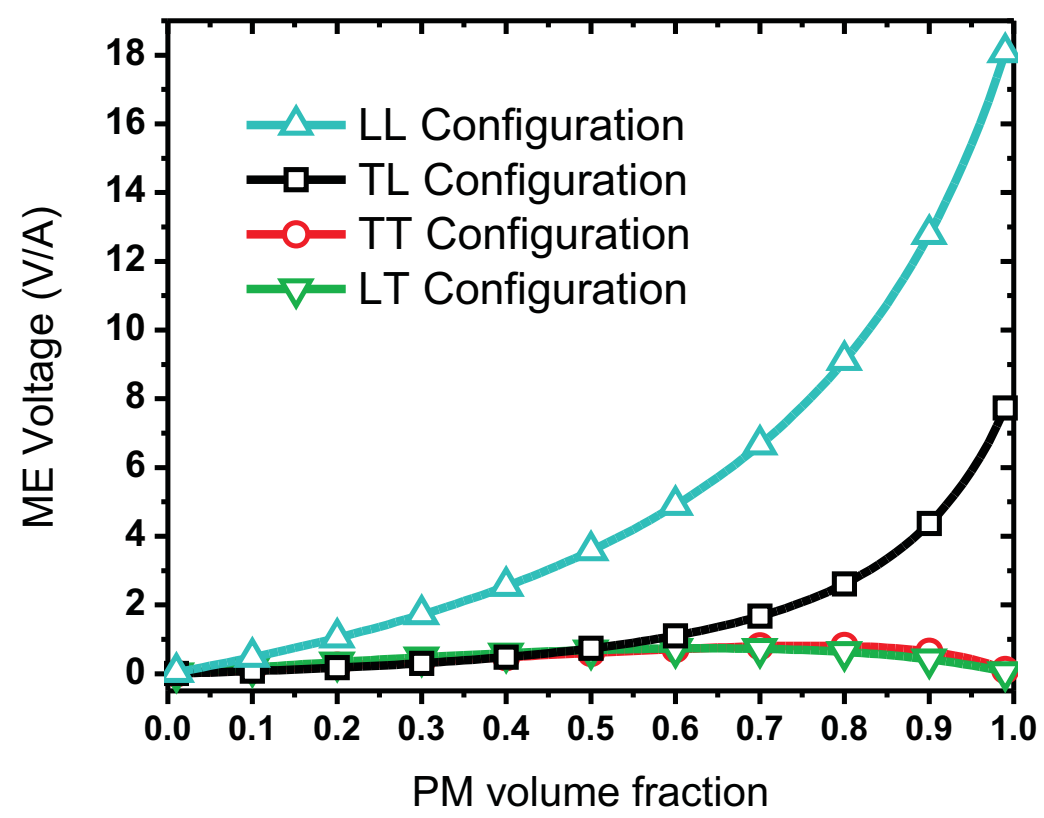

Figure 2.10: ME voltage coefficient of PZT-5H Terfenol-D composite for different PM volume fractions using TT, LT, TL and LL configurations.

for taking into account the conductivity of the PM material without having to introduce additional equations modeling electrical conductivity in the proposed model. In fact, as permittivity $\epsilon^{P M} \rightarrow \infty$, the ME charge coefficient vs. PM volume fraction plot becomes insensitivity to permittivity and the values predicted are close to experimental data for FeBSiC/PZN-PT (Fig. 2.6) for LT configuration. Also, the formulas become much simpler.

For the material systems analyzed, both ME voltage and charge coefficients are maximized when the PM volume fraction $\chi \rightarrow 1$ (in particular configurations). But such design is unrealistic because an infinitely thin layer of PE cannot generate any significant work. Thus, measuring the voltage or the current would be very difficult. In this regard, the newly developed ME coupling factor equations $(2.55,2.55,2.57)$ prove to be very useful in that they provide an indication of the work conversion that can be achieved.

For Terfenol-D/PZT-5H in TT configuration, a reduction of PM volume fraction from $100 \%$ to $90 \%$ results in a reduction of ME charge coefficient from an upper bound of $192 \times$ 
$10^{-9} \mathrm{CA}^{-1} \mathrm{~m}^{-1}$ down to $130 \times 10^{-9} \mathrm{CA}^{-1} \mathrm{~m}^{-1}$, while the ME coupling factor increases from zero to 0.17 . Note that a value of coupling factor $\kappa=1.0$, although practically unattainable, would indicate $100 \%$ work conversion.

In LL configuration, a reduction of PM volume fraction from $100 \%$ to $85 \%$ results in a reduction of ME voltage coefficient from an upper bound $\alpha=18 V A^{-1}$ down to $\alpha=$ $10.7 V A^{-1}$, while the ME coupling factor increases from zero to 0.32 . The formulas presented herein can be used to tune the volume fraction to achieve an acceptable compromise between ME charge, or voltage, and work conversion.

If work conversion needs to be maximized (for transducer applications), the optimum configuration is not TT but LL, at PM volume fraction $73 \%$, yielding a ME coupling factor (i.e., conversion) of 0.36 .

For accurate calculation, it is very important to use the appropriate elastic compliance of the material, which can vary significantly depending on the testing conditions. For example, the compliance in the polarization direction $S_{33}$ of PZT-5H changes from $20.7 \times 10^{-12}$ to $8.9 \times 10^{-12} \mathrm{~m}^{2} \mathrm{~N}^{-1}$ when it is measured at constant $E$ and constant $D$, respectively.

A trade off between voltage and PM volume fraction is evident for LSMO/PZT (Figure 2.3) yielding an optimum PM volume fraction $\chi=65 \%$ in TT configuration. Optimum values like this are dependent on material properties and configuration. Therefore, it is of significant interest for preliminary design and material selection to count with a complete set of formulas such as those proposed in this manuscript to be able to assess voltage, charge, and energy conversion for any of the four laminated configurations, as function of material and geometrical parameters. 


\section{Chapter 3}

\section{Design selection using extrinsic}

\section{charge, voltage, and work-conversion}

\section{factors for laminated magnetoelectric}

\section{composites}

A magnetoelectric composite produces electricity in response to a magnetic field ${ }^{1}$. The voltage, current, and electric power generated by unit of magnetic field applied to the composite define the intrinsic voltage, current, and power conversion factors. Since the magnetostrictive phase of the composite has a higher magnetic permeability than the surrounding medium, a far field magnetic field is not fully utilized due to demagnetization. Thus, novel explicit equations are developed here to calculate the extrinsic voltage, current, and power conversion factors accounting for demagnetization. The proposed formulation is applied to various materials and geometries to illustrate the process of material and device-geometry selection leading to an optimum design.

\footnotetext{
${ }^{1}$ This chapter was published in Smart Materials and Structures journal [75]. (c) IOP Publishing. Reproduced with permission. All rights reserved.
} 


\subsection{Introduction}

Analytical models $[46,47,48,49,55]$ are useful because they can be used to quickly predict approximate values for the expected voltage, charge, or work conversion of the device as a function of material properties for the PE and PM materials as well as a function of the relative volume fractions of the constituents. An analytical model to predict the intrinsic ME response of laminate composites made is presented in Chapter 2 for all four possible laminate configurations (TT, LT, TL, and LL), correctly taking into account the conductivity of the PM phase. However, intrinsic response is not representative of device performance because the far field magnetic field is not fully utilized due to demagnetization, which is caused by the magnetostrictive phase of the composite having a higher magnetic permeability than the surrounding medium. Thus, novel explicit equations are developed herein to calculate the extrinsic voltage, current, and power conversion factors accounting for demagnetization, which unlike for intrinsic properties, requires to account for the geometry of the device ${ }^{2}$.

The ME voltage coefficient provides a indication of the ME material's performance to produce an electric field $E$, and thus voltage $V=E t$ from exposure to a magnetic field $H$, when no work is drawn from the device ${ }^{3}$. Similarly, the ME charge coefficient represents the performance of the ME material to produce an electric displacement $D$, and thus charge $Q=D A$ from exposure to a magnetic field $H$, again when no work is drawn from the device ${ }^{4}$. Finally, the ME coupling factor $\kappa$ yields a measure of the ME material's performance to convert magnetic work into electric work [55, 61, 62].

The formulas for these coefficients require calculation of the dielectric permittivity of the device $\epsilon^{H}$ at constant magnetic field and magnetic permeability of the device $\mu^{E}$ at constant

\footnotetext{
${ }^{2}$ In this work, the composite is called a "material" as long as the geometrical dimensions of the "device" are not relevant. The dimensions are relevant due to demagnetization, requiring the use of the term device to emphasize the dependency of the device behavior with geometrical dimensions. Volume fractions are not dimensions. The PM and PE are called "phases" to emphasize that they are not composites and do not have intrinsic ME effect.

${ }^{3}$ Where $t$ is the total thickness of the device, $E$ is the average electric field over $t$, and $V$ is the voltage measured across the thickness $t$ of the device.

${ }^{4}$ Where $A$ is the area of the device.
} 
electric field, but the well known series/parallel capacitor formulas are not appropriate for PM devices because the application of a magnetic field results in an electrical displacement and vice versa, due to the inherent coupling present in the device. Thus, the methodology proposed in Chapter 2 is used here to derive close form, explicit formulas for their calculation.

The demagnetizing effect has been considered by other researchers, allowing them to obtain extrinsic properties for particular geometries and configurations. In this work, explicit equations are obtained for all four possible configurations (TT,TL,LT,LL) by using the averaged demagnetizing factor approximation. Twenty-eight combinations of seven PM and four PE materials are studied to elucidate the effects of material properties, volume fraction, and device geometry on voltage, charge, and work-conversion. ME properties are calculated to find the optimum PM/PE materials combinations and PM volume fraction $\chi$ for the four configurations (TT, TL, LT, LL). Optimum values are computed for 3 measures of performance, namely ME voltage coefficient $\alpha$, ME charge coefficient $\beta$, and ME coupling factor $\kappa$. Thorough explanations are presented for the trends observed.

\subsection{Materials and constitutive equations}

The PE and PM materials selected for this study are listed in Tables 3.1 and 3.2, respectively. Some properties not available for Galfenol and Metglas are calculated using the same ratios $S_{11} / S_{33}, \nu_{12} / \nu_{31}$ and $q_{31} / q_{33}$ of Terfenol-D. The mechanical properties of CFO ${ }^{5}$ are assumed to be isotropic. The DC magnetic bias used to measure the properties of the PM phase is indicated in Table 3.2. A low magnetic bias is desired for most applications, so that a smaller permanent magnet can be used. This gives a design advantage to Metglas and Galfenol.

The analytical model from Chapter 2 is summarized first. It starts with the multi-physics

\footnotetext{
${ }^{5} \mathrm{CFO}$ stands for $\mathrm{CoFe}_{2} \mathrm{O}_{4}$
} 


\begin{tabular}{c|c|c|c|c|c|c|c|c} 
Property & BTO & PZT-2 & PZT-4 & PZT-5A & PZT-5H & PZT-8 & PVDF & Units \\
\hline$S_{11}$ & 8.6 & 11.6 & 12.3 & 16.4 & 16.5 & 11.5 & 365 & $10^{-12} m^{2} N^{-1}$ \\
\hline$S_{33}$ & 9.1 & 14.8 & 15.5 & 18.8 & 20.7 & 13.5 & 472 & $10^{-12} m^{2} N^{-1}$ \\
\hline$S_{12}$ & -2.6 & -3.33 & -4.05 & -5.74 & -4.78 & -3.7 & -209 & $10^{-12} m^{2} N^{-1}$ \\
\hline$S_{23}$ & -2.7 & -4.97 & -5.31 & -7.22 & -8.45 & -4.8 & -192 & $10^{-12} m^{2} N^{-1}$ \\
\hline$\epsilon_{33} / \epsilon_{0}$ & 1200 & 450 & 1300 & 1700 & 3400 & 1000 & 13 & \\
\hline$\mu_{11} / \mu_{0}$ & 1 & 1 & 1 & 1 & 1 & 1 & 1 & \\
\hline$\mu_{33} / \mu_{0}$ & 1 & 1 & 1 & 1 & 1 & 1 & 1 & \\
\hline$d_{31}$ & -58 & -60.2 & -123 & -171 & -274 & -37 & 21 & $10^{-12} C N^{-1}$ \\
\hline$d_{33}$ & 149 & 152 & 289 & 374 & 593 & 225 & -26 & $10^{-12} C N^{-1}$ \\
\hline
\end{tabular}

Table 3.1: PE material properties $[73,74,76]$. The compliance is measured at constant electric field and the dielectric constant is measured at constant stress.

\begin{tabular}{c|c|c|c|c|c} 
Property & Terfenol-D & Galfenol & Metglas & CFO & Units \\
\hline$S_{11}$ & 44 & 20.3 & 52.7 & 6.5 & $10^{-12} m^{2} N^{-1}$ \\
\hline$S_{33}$ & 38 & 17.5 & 45.5 & 6.5 & $10^{-12} m^{2} N^{-1}$ \\
\hline$S_{12}$ & -11 & -5.1 & -13.2 & -2.37 & $10^{-12} m^{2} N^{-1}$ \\
\hline$S_{23}$ & -16.5 & -7.6 & -19.7 & -2.37 & $10^{-12} m^{2} N^{-1}$ \\
\hline$\epsilon / \epsilon_{0}$ & $\infty$ & $\infty$ & $\infty$ & $\infty$ & \\
\hline$\mu_{33} / \mu_{0}$ & 3 & 260 & 17000 & 2 & \\
\hline$q_{31}$ & -4300 & -23271 & -460353 & 556 & $10^{-12} m A^{-1}$ \\
\hline$q_{33}$ & 8500 & 46000 & 910000 & -1880 & $10^{-12} m A^{-1}$ \\
\hline$k_{31}$ & 0.33 & 0.29 & 0.43 & 0.14 & $10^{-12} m A^{-1}$ \\
\hline$k_{33}$ & 0.71 & 0.61 & 0.92 & 0.47 & $10^{-12} m A^{-1}$ \\
\hline Magnetic Bias & 39.8 & 1.8 & $\sim 0.2$ & $\sim 360$ & $10^{3} \mathrm{Am}^{-1}$ \\
\hline
\end{tabular}

Table 3.2: PM material properties [74, 48, 27], CFO stands for $\mathrm{CoFe}_{2} \mathrm{O}_{4}$. The compliance is measured at constant magnetic field and the magnetic permeability is measured at constant stress. 
constitutive equations

$$
\begin{aligned}
s & =S \sigma+d^{T} E+q^{T} H \\
D & =d \sigma+\epsilon E \\
B & =q \sigma+\mu H
\end{aligned}
$$

where $\sigma$ is the stress tensor, $s$ is the strain tensor, $E$ is the electric field vector, $H$ is the magnetic field vector, $D$ is the electric displacement vector, $B$ is the magnetic flux density vector, $S$ is the compliance tensor (measured at constant electric and magnetic field), $\epsilon$ is the dielectric permittivity tensor (measured at constant stress), $\mu$ is the magnetic permeability tensor (measured at constant stress), $d$ is the $\mathrm{PE}$ charge constant tensor, and $q$ is the $\mathrm{PM}$ constant tensor. These equations describe the behavior of the PM and PE phases. PM materials are actually magnetostrictive, with a nonlinear relationship between strain and magnetic field, but they are customarily treated as linear in the close neighborhood of an applied magnetic bias. Then, the PM constant tensor and all the PM properties are measured at this magnetic bias.

$\mathrm{PE}$ and PM materials display transverse isotropy on a plane normal to the polarization (magnetization) direction, which is denoted by the 3 -axis in this work (Figure 2.1). The polarization and magnetization directions do not coincide for the TL and LT configurations. Therefore, a global coordinate system (Figure 2.2) is used to cast the equations after appropriate coordinate transformation for each of the phases. 
The compliance tensor $S$ is defined as follows [65, (1.91)]

$$
S=\left[\begin{array}{cccccc}
S_{11} & S_{12} & S_{23} & 0 & 0 & 0 \\
S_{12} & S_{11} & S_{23} & 0 & 0 & 0 \\
S_{23} & S_{23} & S_{33} & 0 & 0 & 0 \\
0 & 0 & 0 & S_{44} & 0 & 0 \\
0 & 0 & 0 & 0 & S_{44} & 0 \\
0 & 0 & 0 & 0 & 0 & 2\left(S_{11}-S_{12}\right)
\end{array}\right]
$$

The PE charge constant tensor $d$ is defined as

$$
d=\left[\begin{array}{cccccc}
0 & 0 & 0 & 0 & d_{15} & 0 \\
0 & 0 & 0 & d_{15} & 0 & 0 \\
d_{31} & d_{31} & d_{33} & 0 & 0 & 0
\end{array}\right]
$$

where

$$
\left.\left.d_{i j}=\frac{s_{j}}{E_{i}}\right]_{\sigma=0}=\frac{D_{i}}{\sigma_{j}}\right]_{E=0}
$$

The PM constant tensor $q$ is defined in the same way, where

$$
\left.\left.q_{i j}=\frac{s_{j}}{H_{i}}\right]_{\sigma=0}=\frac{B_{i}}{\sigma_{j}}\right]_{H=0}
$$

The dielectric permittivity $\epsilon$, and magnetic permeability $\mu$, are diagonal tensors defined as

$$
\begin{array}{r}
\epsilon=\epsilon_{i j} \delta_{i j} \\
\mu=\mu_{i j} \delta_{i j}
\end{array}
$$

where $\delta_{i j}$ is the Kronecker symbol and $x_{3}$ is the axis of transverse isotropy, which coincides 
with the direction of polarization or magnetization $\left(\epsilon_{11}=\epsilon_{22}\right.$ and $\left.\mu_{11}=\mu_{22}\right)$.

ME devices can be built using four different configurations (Figure 2.2): transverse magnetization with transverse polarization (TT), longitudinal magnetization with transverse polarization (LT), transverse magnetization with longitudinal polarization (TL), and longitudinal magnetization with longitudinal polarization (LL), as shown in Figure 2.2. Intrinsic voltage, charge, and coupling factors are found by applying boundary conditions representing each case.

For simplicity, only two layers are shown in Figure 2.2, but it is assumed that the actual device is symmetrically laminated. Furthermore, the laminas are thin in comparison to the in-plane dimensions, resulting in a state of plane stress [65] and insignificant shear lag effect [63]. As a result, the stresses are averaged through the thickness of each lamina and the intralaminar/interlaminar shear strains are negligible [65]. Consequently, the ME pair is assumed to be fully effective over its entire area.

The PE phase is most commonly polarized in the transverse direction. Otherwise, an insulator is needed at the interface to prevent charge leakage from the PE through the PM phase, since the latter is highly conductive. For example, a longitudinally polarized PZT was bonded to two magnetostrictive FeBSiC alloy (Metglas) foils, using two Kapton films in between to confine the electric field to the PE phase, thus avoiding leakage [66].

\subsection{Intrinsic properties}

Intrinsic properties are calculated first for the 28 material combinations to establish a baseline for subsequent comparison with the extrinsic properties. This is necessary because extrinsic properties also depend on geometry but intrinsic do not. In this way, it is possible to better elucidate the material effects separately from the geometrical and the demagnetization effects. Intrinsic properties are calculated with the equations presented in Chapter 2. 


\subsubsection{TT configuration}

\section{ME coupling factor}

Power output from the ME composite requires a high ME coupling factor $\kappa$, formally defined as the ratio of electric work harvested to magnetic work applied (Eqn. 2.57). The intrinsic ME coupling factor $\kappa$ of the TT configuration is calculated for the 28 material combinations. The results are shown in Figure 3.1. The highest coupling factor $(\kappa=0.17)$ is obtained for Terfenol-D/PZT-5H composite with PM volume fraction $\chi=85 \%$.

Composites with Terfenol-D and other PZT compositions result in slightly lower ME coupling factors with similar volume fraction dependency. The highest values are obtained for the compositions with higher PE coupling factor. Terfenol-D/BTO has a similar dependency on the PM volume fraction as Terfenol-D/PZT but with smaller values of $\kappa$ due to the smaller PE coupling factor of BTO. Finally, Terfenol-D/PVDF has the smallest ME coupling factor due to the smallest PE coupling factor of PVDF.

For Terfenol-D/PVDF, it can be observed that the optimum ME coupling is obtained for smaller PM volume fraction $\chi$ compared to Terfenol-D/PZT-5H. This is due to the higher compliance of PVDF, which requires a smaller amount of PM phase to obtain the optimum strain.

Regarding the influence of the PM phase, the highest ME coupling is obtained for TerfenolD, even though it does not have the highest PM coupling factor. Although Metglas has the highest PM coupling factor, it does not yield the highest ME coupling factor because of its high magnetic permeability. A high magnetic permeability affects the distribution of $H$ between PM and PE phases which detrimentally affects performance.

The distribution of $H$ between PM and PE phases is obtained from (3.3). The magnetic flux density in the z-direction for the PM phase is

$$
B_{z}^{P M}=2 q_{31}^{P M} \sigma_{x}^{P M}+\mu_{33}^{P M} H_{z}^{P M}
$$




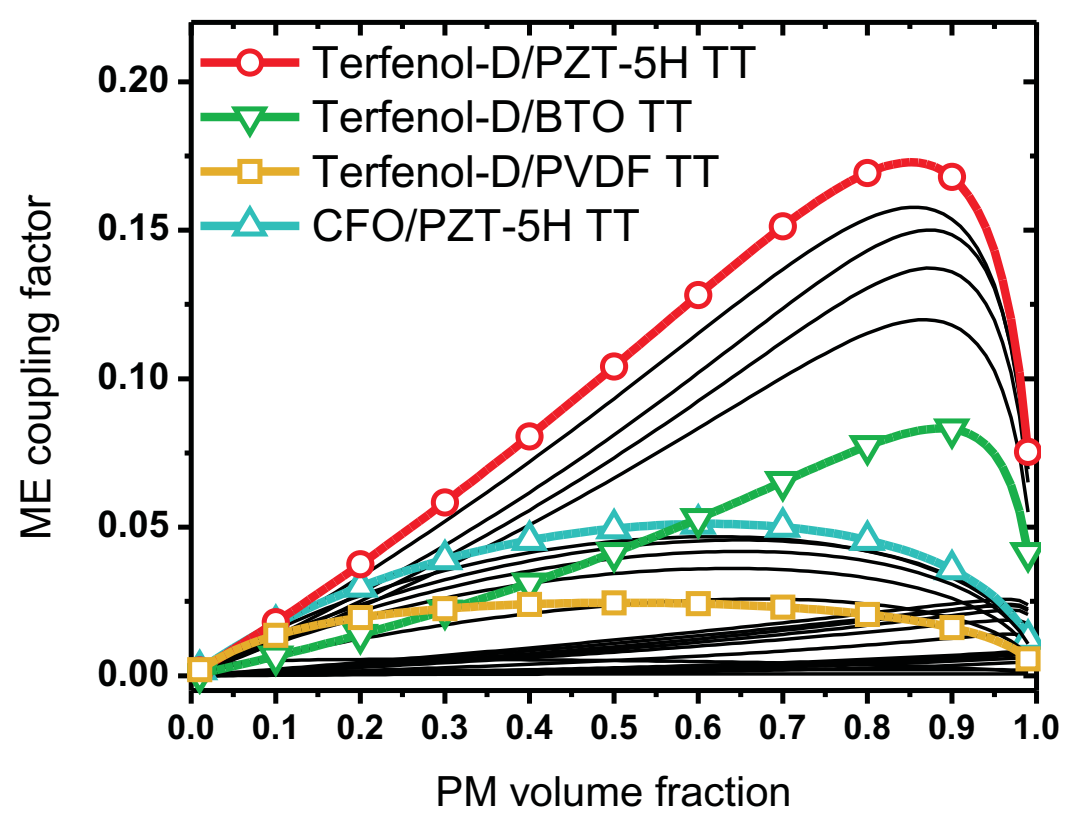

Figure 3.1: ME coupling factor in TT configuration, (dimensionless) for all material combinations. Metglas based composites not labeled because they display very low coupling in this situation (see text). 
and the magnetic flux density for the PE phase is

$$
B_{z}^{P E}=\mu_{33}^{P E} H_{z}^{P E}
$$

When transverse magnetization is used, the magnetic flux density $B$ in both phases are equal $B_{z}^{P E}=B_{z}^{P M}$. Combining equations (3.10) and (3.11) we get

$$
\mu_{33}^{P E} H_{z}^{P E}=2 q_{31}^{P M} \sigma_{x}^{P M}+\mu_{33}^{P M} H_{z}^{P M}
$$

In (3.12), if the PM phase has a magnetic permeability $\mu_{33}^{P M}$ much higher than the PE phase $\mu_{33}^{P E}$, a smaller magnetic field $H_{z}^{P M}$ in the PM phase is observed. This smaller magnetic field reduces the magnetic work harvested by the PM phase. Therefore, for transverse magnetization, a smaller magnetic permeability increases the magnetic field (and the magnetic work harvested) in the PM phase. After Terfenol-D, CFO shows the next higher ME coupling factor, for the same reason.

The effect of the high magnetic permeability on the effective internal magnetic field is further discussed in Section 3.4 to account for demagnetization.

The optimum PM volume fraction with CFO is smaller than with Terfenol-D because $\mathrm{CFO}$, having smaller compliance, generates more stress to drive the PE phase with less PM volume fraction $\chi$ than Terfenol-D.

\section{ME voltage coefficient}

To obtain high open circuit sensitivity requires high ME voltage coefficient $\alpha$ (Equation 2.23). The ME voltage coefficient for the 28 different material combinations in the TT configuration are shown in Figure 3.2. The highest ME voltage $\left(2.3 \mathrm{VA}^{-1}\right)$ is obtained with Terfenol-D/PVDF composite with a PM volume fraction $\chi=41 \%(\kappa=0.024)$.

Another configuration with a high ME voltage coefficient $\left(1.4 V A^{-1}\right)$ is Terfenol-D/PZT-2 composite with PM volume fraction $77 \%$. The optimum $\alpha$ is obtained for higher PM volume 


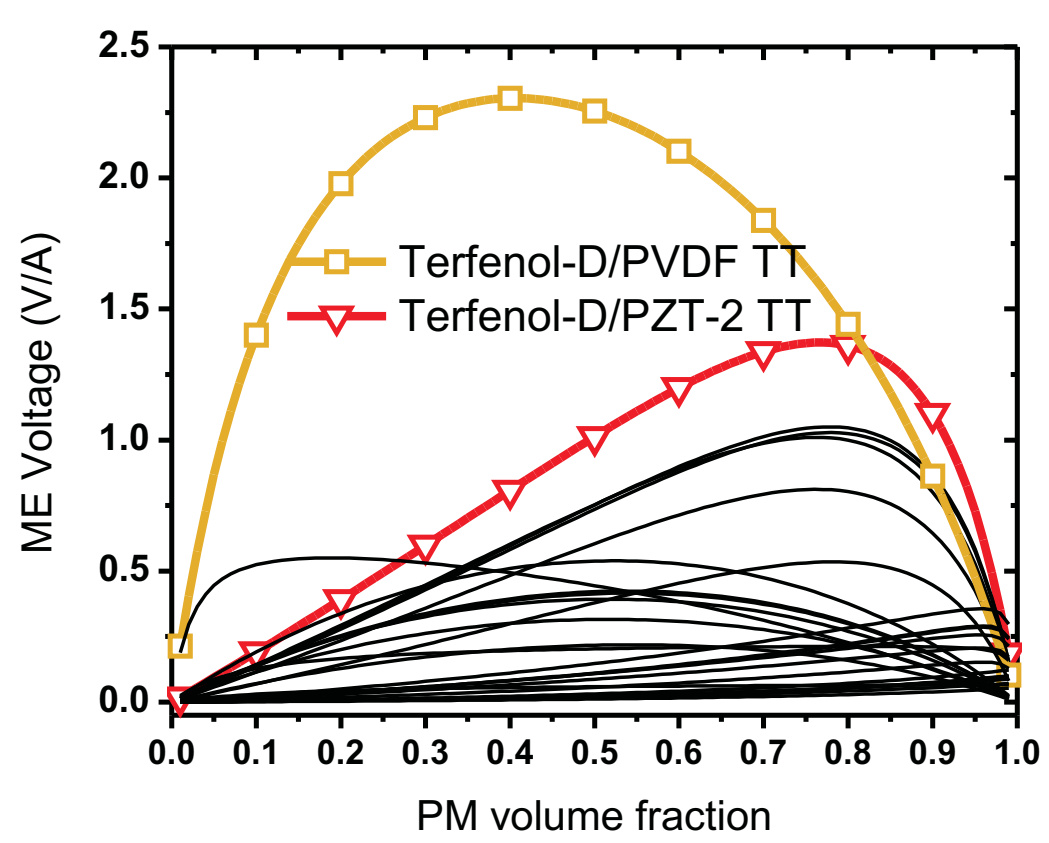

Figure 3.2: ME voltage coefficient (TT configuration) for all material combinations.

fractions because of the higher stiffness of PZT-2, requiring more PM phase to achieve the optimum strain.

\section{ME charge coefficient}

To obtain a high short circuit sensitivity, a high ME charge coefficient $\beta$ is required (Equation 2.25). The ME charge coefficient for the selected composites in the TT configuration are shown in Figure 3.3. In this case, the highest $\beta$ is obtained when $\chi \rightarrow 1$, because the effective area to produce the electric displacement $D$ does not change with the PM volume fraction. Then, $\chi \rightarrow 1$ maximizes the stiffness of the PM phase and thus maximizes the strain transferred to the PE phase. However, $\chi \rightarrow 1$ results in a ME coupling factor $\kappa=0$, making impossible the measurement. Therefore, a compromise between $\beta$ and $\kappa$ is required. The criterion used in this work to achieve this compromise consist in selecting a PM volume fraction that has a high $\beta$ while retaining $70 \%$ of the maximum $\kappa$ for that configuration. In the case of Terfenol-D/PZT-5H, $70 \%$ of the maximum $\kappa$ is 0.12 , which can be obtained 


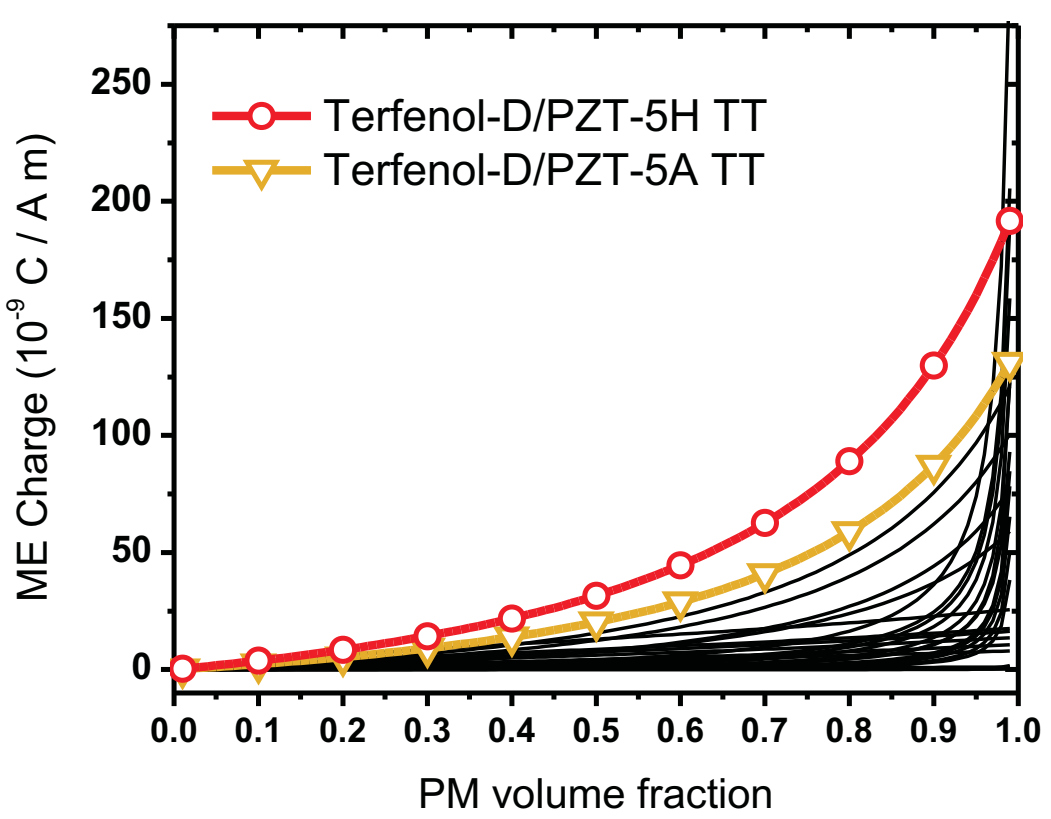

Figure 3.3: ME charge coefficient (TT configuration) for all material combinations.

with $\chi=97 \%$, resulting in $\beta=175 \times 10^{-9} C A^{-1} m^{-1}$. Furthermore, composites using PZT-5H posses a high ME charge coefficient due to the high PE charge coefficient $\left(d_{31}=\right.$ $\left.274 \times 10^{-12} C N^{-1}\right)$ of PZT-5H (Table 3.1).

\subsubsection{LT configuration}

Here the PM is magnetized in the longitudinal direction, i.e., along the longest dimension of the laminate. Longitudinal magnetization results in a increase of the magnetic field in the PM phase compared to transverse magnetization, because (unlike for the TT configuration) the magnetic field is applied at the edges of the PM phase without having to cross the PE phase $\left(H^{P M}=H^{P E}=H_{3}\right)$. This is an advantage for the materials with high magnetic permeability $\mu_{33}^{P M}$ such as Metglas. This effect will be further discussed when the extrinsic properties are calculated using the demagnetizing effect in Section 3.4. Additionally, the PM coupling coefficient $\kappa_{33}$ is higher in the direction of magnetization than $\kappa_{31}$ for the TT configuration. 




Figure 3.4: ME coupling factor (dimensionless) in LT configuration for all material combinations.

\section{ME coupling factor}

The increase of the magnetic field in the PM phase results in a higher magnetic work harvested (compared with transverse magnetization). This can be observed in Figure 3.4 were the ME coupling coefficient of composites (LT configuration) using Metglas show better performance that anything in Figure 3.1.

Regarding the PE phase, the same materials used for the TT configuration give the optimum response. Metglas/PZT-5H composite (LT configuration) with a PM volume fraction of $53 \%$ has a ME coupling factor of 0.23 and Metglas/BTO composite with a PM volume fraction of $64 \%$ has a ME coupling factor of 0.11 .

\section{ME voltage coefficient}

The improvement obtained by magnetization in the longitudinal direction is observed in the ME voltage coefficient as well (Figure 3.5). Here it can be seen that Metglas/PVDF 


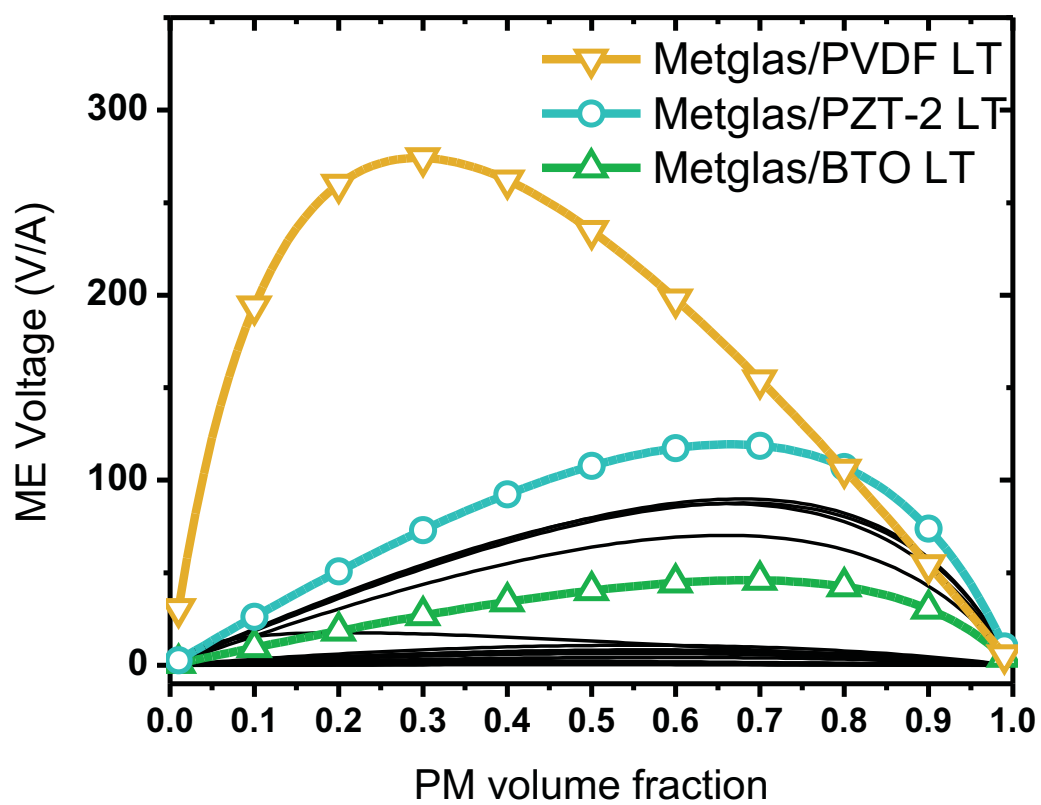

Figure 3.5: ME voltage coefficient for all the material combinations for the LT configuration.

composite has the highest $\alpha=274 V A^{-1}$ with a PM volume fraction of $29 \%(\kappa=0.05)$. Another composite of interest is Metglas/PZT-2, which has smaller ME voltage coefficient $\left(119 \mathrm{VA}^{-1}\right)$ but higher ME coupling factor $(\kappa=0.16)$ for a PM volume fraction of $66 \%$.

\section{ME charge coefficient}

The highest values of ME charge coefficient are obtained for the LT configuration due to the high strain produced by the PM phase and the large effective area of the PE phase. Predictions are shown in Figure 3.6.

If the criterion discussed in Section 3.3.1 is used for the Metglas/PZT-5H composite in the LT configuration, the PM volume fraction to achieve $70 \%$ of the optimum ME coupling is $89 \%$, resulting in a good compromise between ME charge coefficient $(\beta=8393 \times$ $10^{-9} C A^{-1} m^{-1}$ and coupling factor $\left.\kappa=0.16\right)$. An alternative for a lead free material is Metglas/BTO composite with a PM volume fraction of $92 \%$, which results in $\beta=3219 \times$ $10^{-9} C A^{-1} m^{-1}$ and $\kappa=0.08$. 


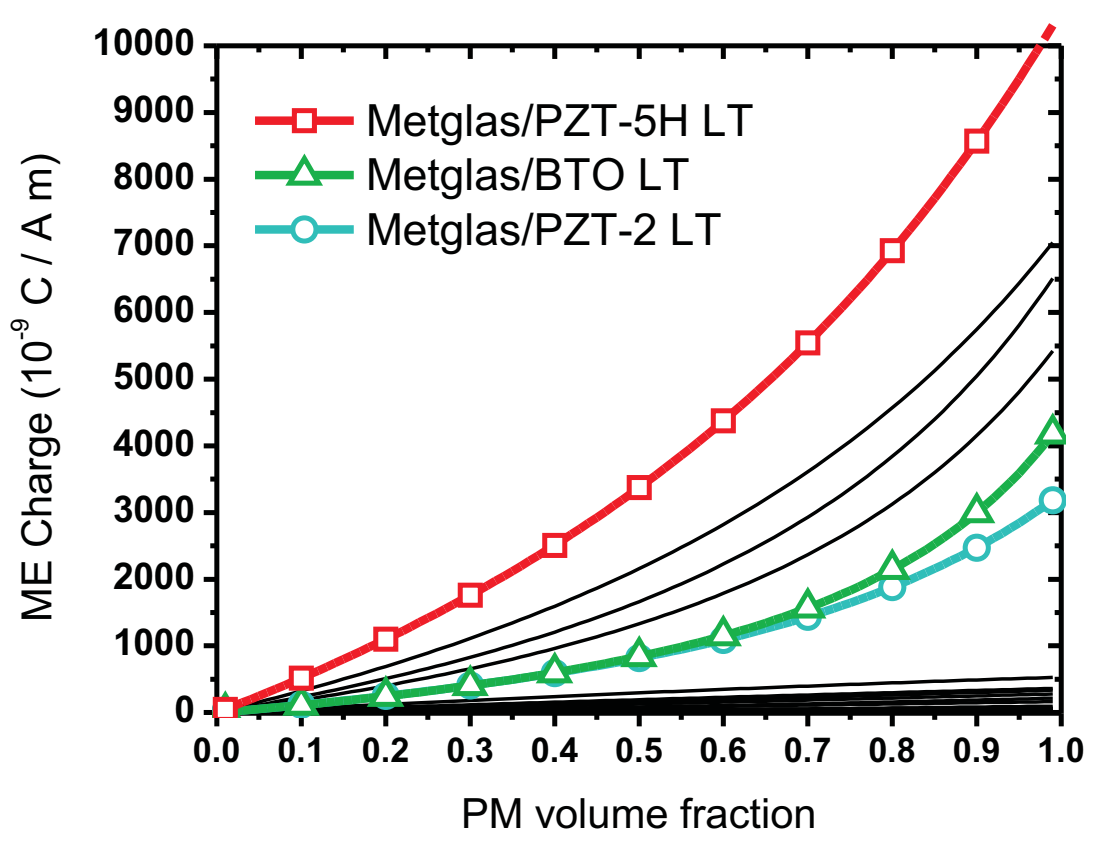

Figure 3.6: ME charge coefficient (LT configuration) for all material combinations.

\subsubsection{TL configuration}

Here the composite is magnetized in the transverse direction but charge/voltage are sensed at the ends of the longitudinal direction. Polarization of the PE phase in the longitudinal direction is more challenging requiring and insulator at the $\mathrm{PM} / \mathrm{PE}$ interface, but ME coupling factor and voltage coefficient are increased. The use of the TL configuration results in smaller ME coefficients and coupling compared to LL and LT configurations. This is due to the smaller magnetic field in the PM phase, as it was discussed in Section 3.3.1. For this reason, this configuration will not be discussed further, but predictions can be obtained using the formulas provided in the Website [69].

\subsubsection{LL configuration}

The highest $\alpha$ and $\kappa$ is predicted when the ME composite is polarized and magnetized in the longitudinal direction, justifying the more complicated fabrication required by longitudinal 


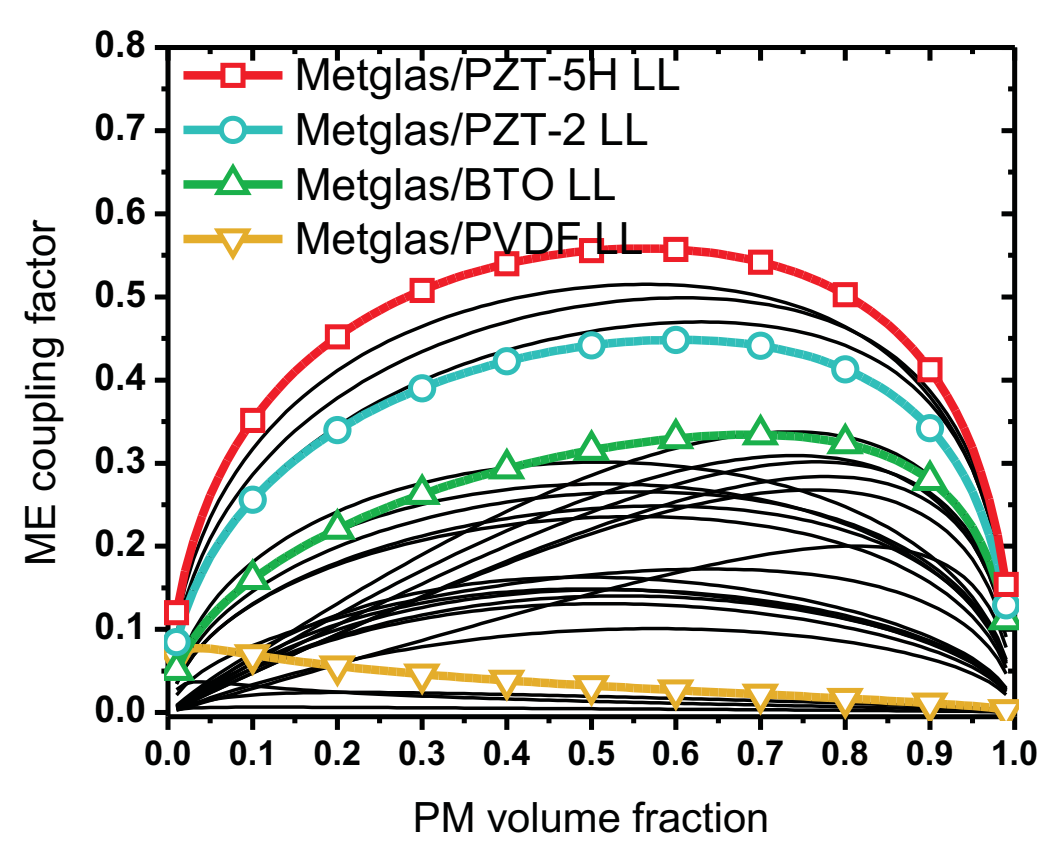

Figure 3.7: ME coupling factor (dimensionless) in LL configuration for all material combinations.

polarization, including an insulator.

\section{ME coupling factor}

Predicted ME coupling factors for LL configuration are shown in Figure 3.7. Metglas/PZT$5 \mathrm{H}$ has the highest $\mathrm{ME}$ coupling factor (0.56) at $57 \% \mathrm{PM}$ volume fraction. This is due to the high PM and PE coupling factors of Metglas and PZT-5H, respectively. For this configuration the coupling factor $k_{33}$ and $k_{31}$ are important because strain is produced in the $\mathrm{x}$ - and y-directions. For a lead free material Metglas/BTO with a PM volume fraction of $69 \%(\kappa=0.33)$ is the best option.

\section{ME charge coefficient}

Predictions for the ME charge coefficient can be seen in Figure 3.8. Metglas/PZT-5H composite has a ME charge coefficient of $4246 \times 10^{-9} C A^{-1} m^{-1}$ and $\kappa=0.56$ for a PM volume 


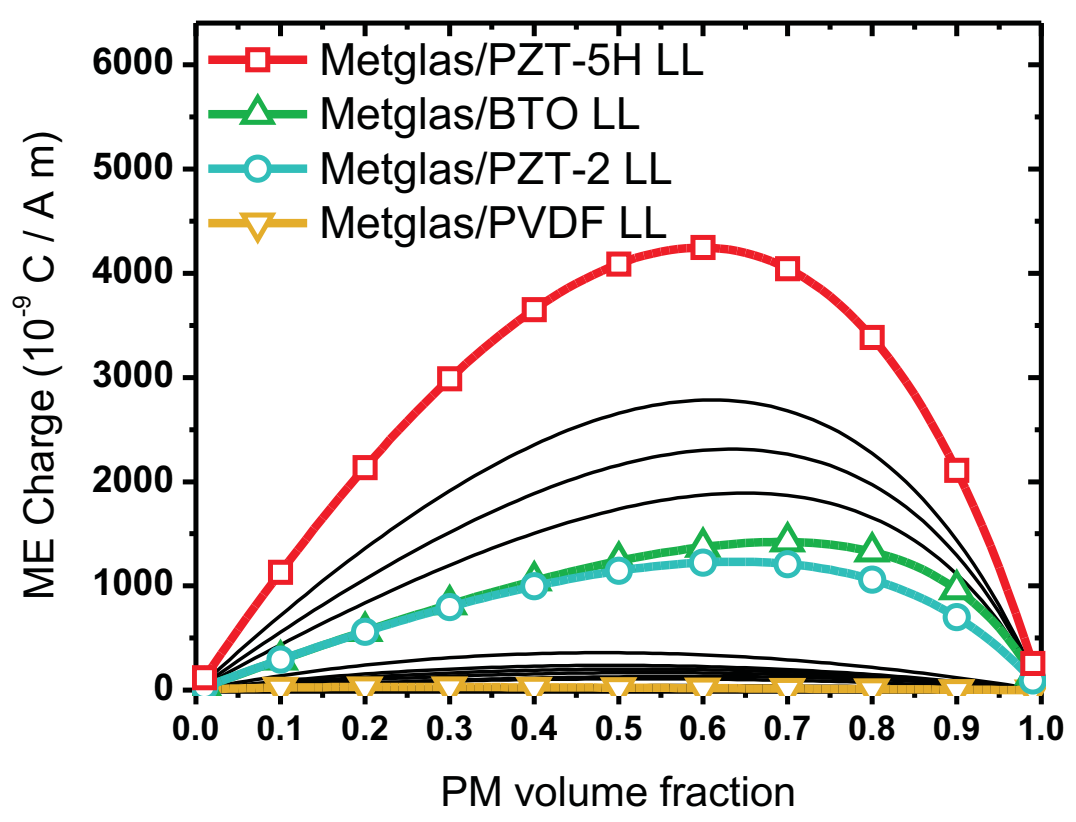

Figure 3.8: ME charge coefficient (LL configuration) for all material combinations.

fraction of $60 \%$. The $\beta$ is smaller than the value for the LT configuration but the coupling coefficient associated is three times higher. Metglas/BTO composite is a good alternative to PZT, yielding $\beta=1423 \times 10^{-9} C A^{-1} m^{-1}$ and $\kappa=0.33$ for a PM volume fraction of $69 \%$.

\section{ME voltage coefficient}

The ME voltage coefficient for the LL configuration can be seen in Figure 3.9. Metglas/PZT2 composite reaches a good compromise (according to the established criterion) between the ME voltage coefficient and the ME coupling factor at PM volume fraction of $92 \%$, resulting in $\alpha=2705 V A^{-1}$ and $\kappa=0.32$.

Metglas/PVDF composite shown an unusual behavior as a function of the PM volume fraction. For $\chi<0.4$, increasing the PM volume fraction increases the ME voltage coefficient, but after $40 \%$ the ME voltage coefficient remains practically constant. This is due to the fact that the elastic modulus of PVDF is more than six times smaller than the elastic modulus of Metglas. As a result, only a small volume fraction of Metglas is required to produce high 


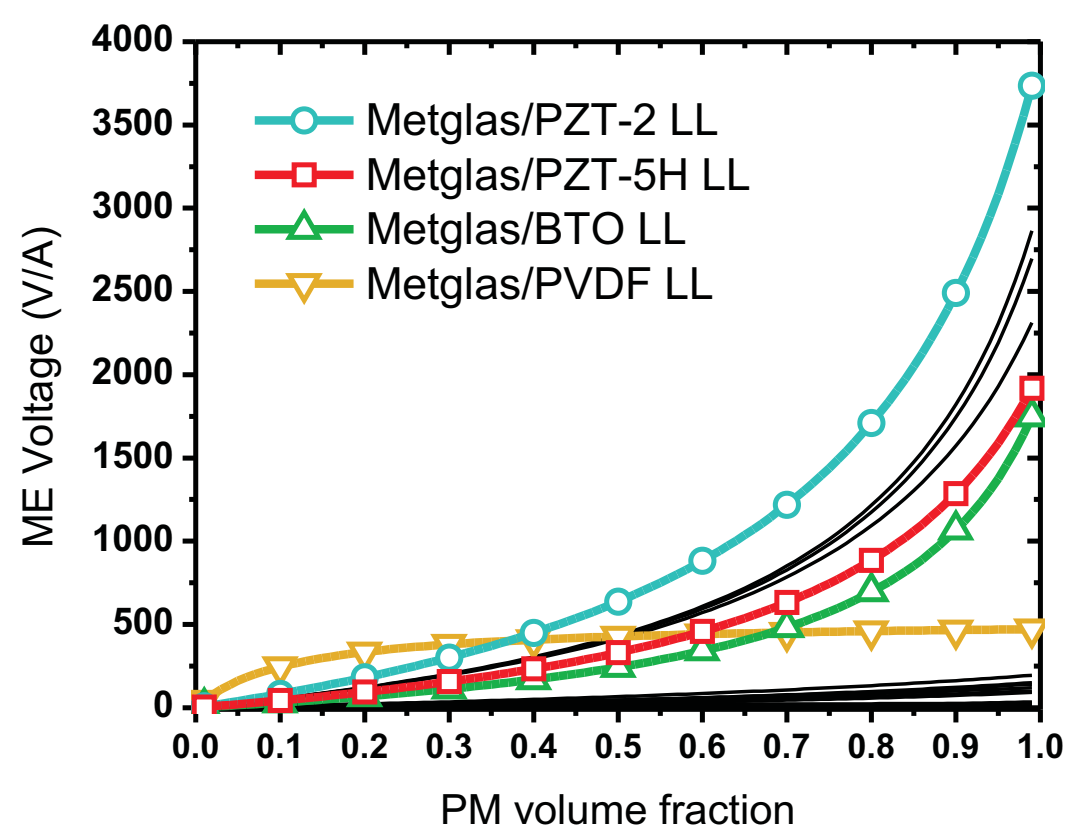

Figure 3.9: ME voltage coefficient for all the material combinations for the LL configuration.

strain in the PVDF layer and higher volume fractions of Metglas practically do not increase the strain in the PVDF.

A similar effect can be seen in the ME coupling factor (Figure 3.7). The compromise between the ME voltage coefficient and coupling factor for Metglas/PVDF in LL configuration is obtained for PM volume fraction of $22 \%$, resulting in $\alpha=345 V A^{-1}$ and $\kappa=0.05$. Another lead free option is Metglas/BTO composite with a PM volume fraction of $94 \%$ having a ME voltage of $1304 V A^{-1}$ and a coupling factor of 0.24 .

\subsubsection{Summary of intrinsic properties}

Composites with high open circuit sensitivity are shown in Table 3.3. Longitudinal magnetization yields higher coefficients due to the higher strain produced and Metglas is the best PM phase for this configuration. The first three composites require the more complex longitudinal polarization but produce higher ME voltage coefficient $(\alpha)$. For the first three composites, a PM volume fraction lower than the optimum $(\chi \rightarrow 1)$ is selected to avoid 


\begin{tabular}{c|c|c|c|c|c|c|c} 
PM Phase & Metglas & Metglas & Metglas & Metglas & Metglas & Metglas & n/a \\
\hline PE Phase & PZT-2 & PVDF & BTO & PVDF & PZT-2 & BTO & n/a \\
\hline Configuration & LL & LL & LL & LT & LT & LT & n/a \\
\hline$\chi$ & $92 \%^{a}$ & $2 \%^{a}$ & $94 \%^{a}$ & $29 \%$ & $66 \%$ & $69 \%$ & \\
\hline$\alpha$ & 2705 & 345 & 1304 & 274 & 119 & 46 & $V / A$ \\
\hline$\kappa$ & 0.32 & 0.05 & 0.24 & 0.05 & 0.16 & 0.11 & \\
\hline$\beta$ & 595 & 31 & 686 & 43 & 1290 & 1522 & $10^{-9} C /(A m)$ \\
\hline
\end{tabular}

Table 3.3: ME composites with high voltage coefficient for open circuit sensors application. ${ }^{a}$ The optimum PM volume fraction $(\chi \rightarrow 1)$ is not used to avoid $\kappa=0$, but PM volume fraction is selected to retain $70 \%$ of the maximum ME coupling factor for each configuration.

$\kappa=0$. The highest performance PE phase is PZT-2. For lead free composites, BTO followed by PVDF provide the best performances. High open circuit sensitivity is the only application where PVDF shows good performance because its small PE charge coefficient and coupling factor results in small $\beta$ and $\kappa$.

Composites with better closed circuit sensitivity are shown in Table 3.4. Transverse polarization results in a higher closed circuit sensitivity due to the higher area at which the electric displacement is produced. $\mathrm{PZT}-5 \mathrm{H}$ results in the highest $\beta$ between the PE materials selected due to its high PE charge coefficient $(d)$. Smaller PM volume fraction than the optimum $(\chi \rightarrow 1)$ is selected to achieve a good trade off between $\kappa$ and $\beta$ for the LL configuration.

Composites with longitudinal polarization have about half the ME charge coefficient compared with transverse polarization. For the PM phase, Metglas in longitudinal magnetization has the highest performance. Lead free ME composites for closed circuit applications can be fabricated using BTO with the disadvantage of decreasing $\beta$ to about a third.

Composites with better ME coupling factor are shown in Table 3.5. ME harvesters require that most of the harvested magnetic work is transformed to electric work, requiring a structure with high ME coupling factor $(\kappa)$. Among the materials considered in this study, the composite that better satisfies this is Metglas/PZT-5H in LL configuration with a PM volume fraction of $60 \%$, which results in $\kappa=0.56$ (Table 3.5).

Composites with transverse polarization have $\kappa$ more than two times smaller due to the 


\begin{tabular}{c|c|c|c|c|c} 
PM Phase & Metglas & Metglas & Metglas & Metglas & $\mathrm{n} / \mathrm{a}$ \\
\hline PE Phase & PZT-5H & BTO & PZT-5H & BTO & $\mathrm{n} / \mathrm{a}$ \\
\hline Configuration & LT & LT & LL & LL & $\mathrm{n} / \mathrm{a}$ \\
\hline$\chi$ & $89 \%^{a}$ & $92 \%^{a}$ & $60 \%$ & $69 \%$ & \\
\hline$\beta$ & 8393 & 3219 & 4246 & 1423 & $10^{-9} C /(A m)$ \\
\hline$\kappa$ & 0.16 & 0.08 & 0.56 & 0.33 & \\
\hline$\alpha$ & 45 & 26 & 458 & 465 & $V / A$ \\
\hline
\end{tabular}

Table 3.4: ME composites with high charge coefficient for closed circuit sensor applications. ${ }^{a}$ The optimum PM volume fraction $(\chi \rightarrow 1)$ is not used to avoid $\kappa=0$, but PM volume fraction is selected to retain $70 \%$ of the maximum ME coupling factor for each configuration.

\begin{tabular}{c|c|c|c|c|c} 
PM Phase & Metglas & Metglas & Metglas & Metglas & $\mathrm{n} / \mathrm{a}$ \\
\hline PE Phase & PZT-5H & BTO & PZT-5H & BTO & $\mathrm{n} / \mathrm{a}$ \\
\hline Configuration & LL & LL & LT & LT & $\mathrm{n} / \mathrm{a}$ \\
\hline$\chi$ & $60 \%$ & $69 \%$ & $53 \%$ & $64 \%$ & \\
\hline \hline$\kappa$ & 0.56 & 0.33 & 0.23 & 0.11 & \\
\hline$\alpha$ & 458 & 465 & 66 & 46 & $V / A$ \\
\hline$\beta$ & 4246 & 1423 & 3657 & 1305 & $10^{-9} C /(A m)$ \\
\hline
\end{tabular}

Table 3.5: ME composites properties with high coupling factor for harvesters applications.

smaller $k_{31}$ of the PE phase. Using lead free BTO in the LL configuration reduces the ME coupling factor to 0.33 .

\subsection{Extrinsic ME properties}

When a device is exposed to an external magnetic field $H_{0}$, the internal magnetic field $H_{3}$ is reduced by the demagnetization field

$$
H_{3}^{\prime}=H_{0}+H_{d}=H_{0}-N_{3} M
$$

where $H_{d}$ is the demagnetizing field, $N_{3}$ is the demagnetizing factor in direction 3 , and $M$ is the magnetization. The magnetization can be written as follows [77]

$$
M=\frac{H_{0}\left(\mu_{r}-1\right)}{1+N_{3}\left(\mu_{r}-1\right)}
$$


resulting in

$$
H_{3}^{\prime}=H_{0} \frac{\mu_{0}}{\mu_{0}+N_{3}\left(\mu-\mu_{0}\right)}
$$

where $H_{3}^{\prime}$ is the magnetic field inside the composite material when the magnetic behavior of the PE phase is assumed to be blended with the surrounding medium (the PM phase is the only phase which has an effect on demagnetization, not the PE). The ' symbol is used to differentiate $H_{3}^{\prime}$ from $H_{3}$, which is the magnetic field inside the composite material when the magnetic behavior of the PE phase is homogenized with the PM phase, as in [55]. We shall see in Section 3.4.1 and 3.4.2 that $H_{3}^{\prime}=H_{3}$ for longitudinal magnetization but $H_{3}^{\prime} \neq H_{3}$ for transverse magnetization.

Furthermore, $H_{0}$ is the externally applied magnetic field, $\mu$ is the magnetic permeability of the material, $\mu_{0}$ is the magnetic permeability of vacuum, $\mu_{r}=\mu / \mu_{0}$ and $N_{3}$ is the demagnetizing factor in the 3 direction (the direction in which the magnetic field is applied). $N_{3}$ is a function of the geometry of the device.

The demagnetizing factor has values between 0 and 1 . When $N_{3}=0, H_{3}^{\prime}=H_{0}$ and when $N_{3}=1, H_{3}^{\prime}=H_{0} / \mu_{r}$. Devices with high demagnetizing factor $N_{3}$ made with materials having high relative permeability $\mu_{r}$ experience the highest reduction of internal magnetic field $H_{3}^{\prime}$. While intrinsic material performance is independent of geometry, the extrinsic device performance, i.e., the voltage generated for a given magnetic field, is a function of the dimensions of the device as well as intrinsic performance. The extrinsic performance can be calculated in terms of the intrinsic material performance and the demagnetizing factor for particular device dimensions $[70,71]$.

The demagnetization factor satisfies the condition $N_{1}+N_{2}+N_{3}=1$. The simplest case is for an sphere, for which $N_{i}=1 / 3$ and constant in all directions. In the case of an infinite plate, the out of plane demagnetizing factor is 1 and the in-plane demagnetizing factor is 0 . For example, for a thin ME laminate in the shape of a large square plate, the TT configuration suffers because $N_{3} \rightarrow 1$, but the LT and LL configurations have the advantage 
because $N_{3} \rightarrow 0$.

The demagnetizing factor for non-infinite plates as a function of position can be calculated using the equations of Joseph and Schlomann [70]. The averaged demagnetization factor can be obtained using the expressions derived by Aharoni [71]. Thus, the dimensions of the ME device can be optimized taking into account the demagnetizing factor $N_{3}$. The optimal dimensions would yield $N_{3} \rightarrow 0$.

For the case of composites with longitudinal magnetization (LT and LL), the thickness has to be considerably smaller than the in-plane dimensions to approach $N \rightarrow 0$. For composites with transverse magnetization (TT and TL), measured performance, rather than intrinsic performance, may be significantly reduced due to high demagnetization factor [72].

The demagnetizing factor $N_{3}$ is constant inside an ellipsoid but not inside a cuboid [70]. To take into account the variation of the demagnetizing factor at different positions, the constitutive equations have to be solved at every point and integrated trough the volume, drastically increasing the complexity of the analysis. To simplify the analysis, in this work an approximation is used; the demagnetizing factor is averaged through the volume. This allows to us obtain explicit formulas for the ME coefficients.

The averaged demagnetizing factor is calculated using the following formula [71]

$$
\begin{aligned}
\pi N_{3}= & \frac{b^{2}-c^{2}}{2 b c} \ln \left(\frac{\sqrt{a^{2}+b^{2}+c^{2}}-a}{\sqrt{a^{2}+b^{2}+c^{2}}+a}\right)+\frac{a^{2}-c^{2}}{2 a c} \ln \left(\frac{\sqrt{a^{2}+b^{2}+c^{2}}-b}{\sqrt{a^{2}+b^{2}+c^{2}}+b}\right) \\
& +\frac{b}{2 c} \ln \left(\frac{\sqrt{a^{2}+b^{2}}+a}{\sqrt{a^{2}+b^{2}}-a}\right)+\frac{a}{2 c} \ln \left(\frac{\sqrt{a^{2}+b^{2}}+b}{\sqrt{a^{2}+b^{2}}-b}\right) \\
& +\frac{c}{2 a} \ln \left(\frac{\sqrt{b^{2}+c^{2}}-b}{\sqrt{b^{2}+c^{2}}+b}\right)+\frac{c}{2 b} \ln \left(\frac{\sqrt{a^{2}+c^{2}}-a}{\sqrt{a^{2}+c^{2}}+a}\right) \\
& +2 \arctan \left(\frac{a b}{c \sqrt{a^{2}+b^{2}+c^{2}}}\right)+\frac{a^{3}+b^{3}-2 c^{3}}{3 a b c} \\
& +\frac{a^{2}+b^{2}-2 c^{2}}{3 a b c} \sqrt{a^{2}+b^{2}+c^{2}}+\frac{c}{a b}\left(\sqrt{a^{2}+c^{2}}+\sqrt{b^{2}+c^{2}}\right) \\
& -\frac{\left(a^{2}+b^{2}\right)^{3 / 2}+\left(b^{2}+c^{2}\right)^{3 / 2}+\left(c^{2}+a^{2}\right)^{3 / 2}}{3 a b c}
\end{aligned}
$$

where $c$ is the length of the PM phase in the direction of the applied field (direction 3), and 
$a, b$, are the dimensions of the PM phase perpendicular to the applied field.

Equation (3.16) does not apply to a composite with two or more different phases. Fortunately, since the magnetic permeability of the PE phase $\mu^{P E} \approx \mu_{0}$, it can be blended with the surrounding medium. Then, the dimensions used in Equation 3.16 correspond to the dimensions of the PM lamina only. Additionally, the magnetic permeability of the material used in Equation 3.15 has to be that of the PM phase only. This will be further discussed for each extrinsic property calculated.

\subsubsection{Extrinsic ME voltage coefficient}

The extrinsic ME voltage coefficient $\hat{\alpha}$, which depends in the actual geometry of the composite is defined as

$$
\left.\hat{\alpha}=\frac{E}{H_{0}}\right]_{D=0}
$$

The intrinsic ME voltage coefficient for the homogenized composite subject to magnetic field $H_{3}$ (see Section 3.4), is defined as

$$
\left.\alpha=\frac{E}{H_{3}}\right]_{D=0}
$$

At this point is convenient to define the intrinsic ME voltage coefficient for the homogenized composite subject to magnetic field $H_{3}^{\prime}$ (which is relevant in the magnetic phase only), as follows

$$
\left.\alpha^{\prime}=\frac{E}{H_{3}^{\prime}}\right]_{D=0}
$$

which is similar to $\alpha$ but with the magnetic field applied at the boundaries of the magnetic phase only. There are two different cases. First, when longitudinal magnetization is used, $H_{3}^{\prime}=H_{3}$ and $\alpha^{\prime}=\alpha$. The second case is when the composite is magnetized in the transverse 
direction, for which the magnetic field $H_{3}$ through the composite is

$$
H_{3}=\chi H^{P M}+(1-\chi) H^{P E}
$$

Thus, $\alpha^{\prime}$ has to be calculated using (3.19) for all the with transverse magnetization. Finally, the extrinsic voltage coefficient $\hat{\alpha}$ can be obtained as follows

$$
\begin{aligned}
\left.\left.\frac{\hat{\alpha}}{\alpha^{\prime}}=\frac{E}{H_{0}} \times \frac{H_{3}^{\prime}}{E}\right]_{D=0}=\frac{H_{3}^{\prime}}{H_{0}}=\right]_{D=0} \\
\left.\frac{\hat{\alpha}}{\alpha^{\prime}}=\frac{\mu_{0}}{\mu_{0}+N_{3}\left(\mu^{\prime}-\mu_{0}\right)}\right]_{D=0}
\end{aligned}
$$

where $\left.\mu^{\prime}\right]_{D=0}$ is the magnetic permeability of the PM phase mechanically clamped to the PE phase and with $D=0$, which can be calculated as

$$
\left.\left.\left.\mu^{\prime}\right]_{D=0}=\frac{B_{3}^{\prime}}{H_{3}^{\prime}}\right]_{D=0}=\frac{B^{P M}}{H^{P M}}\right]_{D=0}
$$

where $B_{3}^{\prime}$ is the magnetic flux in the magnetic phase only.

Analytical expressions for $\left.\mu^{\prime}\right]_{D=0}$ in all four configurations are developed in this work by solving the constitutive equations subjected to appropriate boundary conditions for each configuration. For the LL configuration the following expression is obtained 


$$
\begin{aligned}
\left.\mu_{L L}\right]_{D=0} & =\frac{\epsilon_{33}^{P E}\left(A_{1}+B_{1}+C_{1}+D_{1}\right)+\chi\left(E_{1}+F_{1}+G_{1}\right)}{\epsilon_{33}^{P E}\left(H_{1}+I_{1}\right)+\chi\left(J_{1}+K_{1}\right)} \\
A_{1} & =\left(q_{33}^{P M}\right)^{2}(\chi-1)\left(S_{11}^{P M}(\chi-1)-S_{11}^{P E} \chi\right)-2 q_{31}^{P M} q_{33}^{P M}(\chi-1)\left(S_{23}^{P M}(\chi-1)-S_{23}^{P E} \chi\right) \\
B_{1} & =\left(q_{31}^{P M}\right)^{2}(\chi-1)\left(S_{33}^{P M}(\chi-1)-S_{33}^{P E} \chi\right)+\mu_{33}^{P M}\left(\left(S_{23}^{P M}\right)^{2}-S_{11}^{P M} S_{33}^{P M}\right. \\
C_{1} & =-\left(2 S_{23}^{P M}\left(S_{23}^{P M}-S_{23}^{P E}\right)+S_{11}^{P M}\left(S_{33}^{P E}-2 S_{33}^{P M}\right)+S_{11}^{P E} S_{33}^{P M}\right) \chi \\
D_{1} & \left.\left.=\left(\left(S_{23}^{P E}-S_{23}^{P M}\right)^{2}-\left(S_{11}^{P E}-S_{11}^{P M}\right)\left(S_{33}^{P E}-S_{33}^{P M}\right)\right) \chi^{2}\right)\right) \\
E_{1} & =\left(d_{33}^{P E}\right)^{2}\left(\left(q_{31}^{P M}\right)^{2}(\chi-1)+\mu_{33}^{P M}\left(S_{11}^{P M}+S_{11}^{P E} \chi-S_{11}^{P M} \chi\right)\right) \\
F_{1} & =-2 d_{31}^{P E} d_{33}^{P E}\left(q_{31}^{P M} q_{33}^{P M}(\chi-1)+\mu_{33}^{P M}\left(S_{23}^{P M}+S_{23}^{P E} \chi-S_{23}^{P M} \chi\right)\right) \\
G_{1} & =\left(d_{31}^{P E}\right)^{2}\left(\left(q_{33}^{P M}\right)^{2}(\chi-1)+\mu_{33}^{P M}\left(S_{33}^{P M}+S_{33}^{P E} \chi-S_{33}^{P M} \chi\right)\right) \\
H_{1} & =\left(S_{23}^{P M}\right)^{2}-S_{11}^{P M} S_{33}^{P M}-\left(S_{33}^{P E} S_{11}^{P M}+2 S_{23}^{P M}\left(-S_{23}^{P E}+S_{23}^{P M}\right)+\left(S_{11}^{P E}-2 S_{11}^{P M}\right) S_{33}^{P M}\right) \chi \\
I_{1} & =\left(\left(S_{23}^{P E}-S_{23}^{P M}\right)^{2}-\left(S_{11}^{P E}-S_{11}^{P M}\right)\left(S_{33}^{P E}-S_{33}^{P M}\right)\right) \chi^{2} \\
J_{1} & =2 d_{31}^{P E} d_{33}^{P E}\left(S_{23}^{P M}(\chi-1)-S_{23}^{P E} \chi\right)+\left(d_{33}^{P E}\right)^{2}\left(S_{11}^{P M}+S_{11}^{P E} \chi-S_{11}^{P M} \chi\right) \\
K_{1} & =\left(d_{31}^{P E}\right)^{2}\left(S_{33}^{P M}+S_{33}^{P E} \chi-S_{33}^{P M} \chi\right)
\end{aligned}
$$

Equation (3.23) and a similar expression for the LT configuration (not shown in this paper) can be found in the Website [69].

Values of extrinsic ME voltage coefficient are calculated for Metglas/PZT-2 composite in LL configuration and the results are shown in Figure 3.10. These results were calculated using a composite with equal length and width (square in-plane) and various thicknesses. The aspect ratio $r$ is defined as follows

$$
r=\frac{c}{t}=\frac{a}{t}
$$

where $a$ is the width of the composite, $c$ is the length of the composite (in the direction of the applied magnetic field, for the longitudinal magnetization) and $t$ is the total thickness of the 
composite. Therefore, the thickness of the PM phase is obtained in terms of the PM volume fraction as $b=\chi t$. It can be seen in Figure 3.10 that when the aspect ratio is reduced, the extrinsic ME voltage decreases.

Intrinsic properties are obtained when $r \rightarrow \infty$ because the demagnetizing factor approaches zero $\left(N_{3} \rightarrow 0\right)$. Very high values for the aspect ratio are required to preserve the intrinsic properties in the case of Metglas. This is due to its high magnetic permeability, making it very sensitive to demagnetizing effects. For example, if the aspect ratio is $r=10^{4}$ for Metglas/PZT-2 LL, the extrinsic ME voltage is $\hat{\alpha}=581 V A^{-1}$, which is considerably smaller than the intrinsic value of $\alpha=2705 \mathrm{VA}^{-1}$, where both values are calculated using $\chi=92 \%$ to retain $70 \%$ of the maximum intrinsic ME coupling factor. In practice, aspect ratios of $r=10$ are preferred to facilitate the fabrication of the device. In such case, the extrinsic ME voltage coefficient decreases to $\hat{\alpha}=2.4 \mathrm{VA}^{-1}$, which is $10^{3}$ times smaller than the intrinsic value.

Since ME voltage decreases so drastically for Metglas/PZT-2 for small aspect ratios, other materials with smaller magnetic permeability are investigated to see if they can sustain higher extrinsic ME voltage. Results for all material combinations in LL configuration are shown in Figure 3.11 for aspect ratio $r=10$. It can be seen that composites with Terfenol-D have the highest extrinsic ME voltage due to their smaller magnetic permeability. With regards to the PE phase, PZT-2 allows for the highest extrinsic ME voltage. Terfenol-D/PZT-2 has the highest extrinsic ME voltage $\hat{\alpha}=26 V A^{-1}$ for a PM volume fraction of $\chi=95 \%$. Using Galfenol instead of Terfenol-D results in a lower $\hat{\alpha}$ due to the higher magnetic permeability of Galfenol.

The effect of the aspect ratio can be seen in Figure 3.12. The demagnetizing effect is stronger for materials with higher magnetic permeability, resulting in higher decrease of the extrinsic ME voltage. It can be seen in Figure 3.12 that Metglas/PZT-2 has the highest $\hat{\alpha}$ for aspect ratios higher than $r>1200$. For small aspect ratios $(r<90)$, Terfenol-D/PZT-2 shows the highest extrinsic ME voltage, and between these aspect ratios, Galfenol/PZT-2 


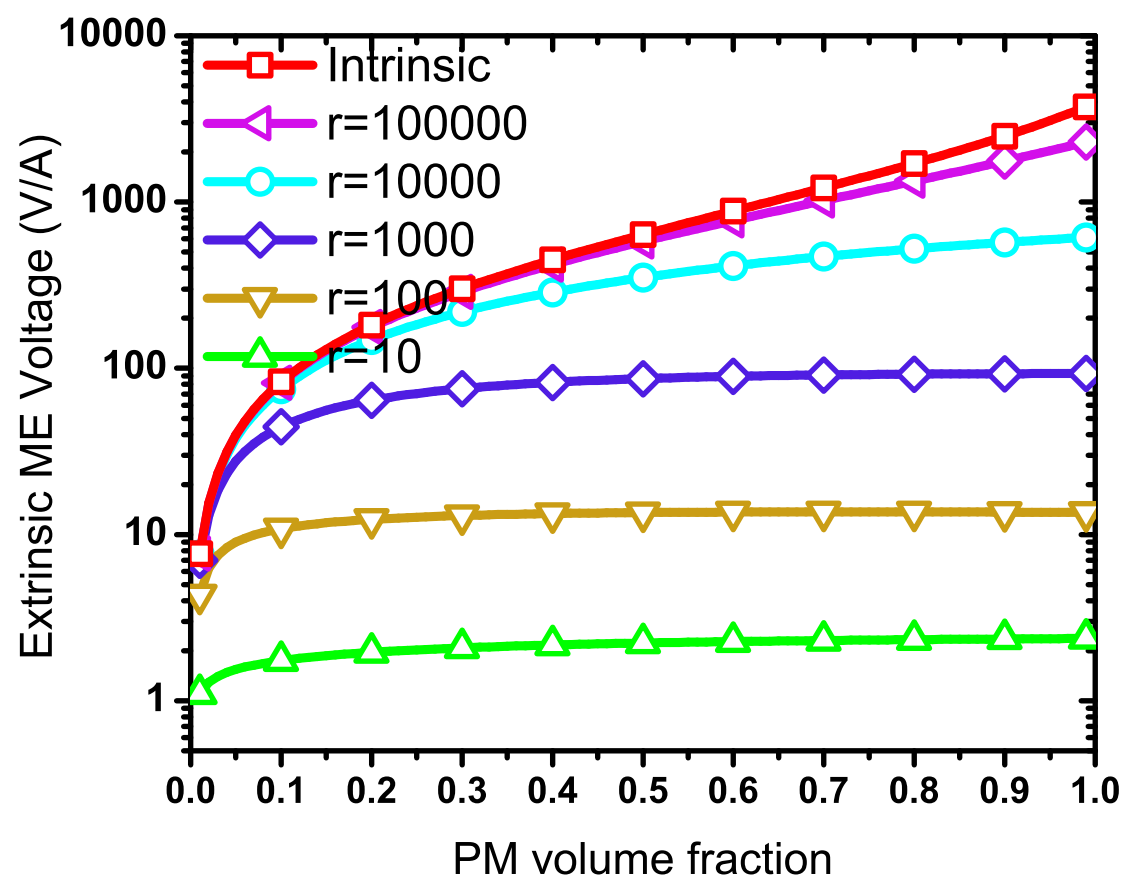

Figure 3.10: Extrinsic ME voltage coefficient for Metglas/PZT-2 in LL configuration for aspect ratios of 10,100,10000, and $r \rightarrow \infty$ (intrinsic value). 


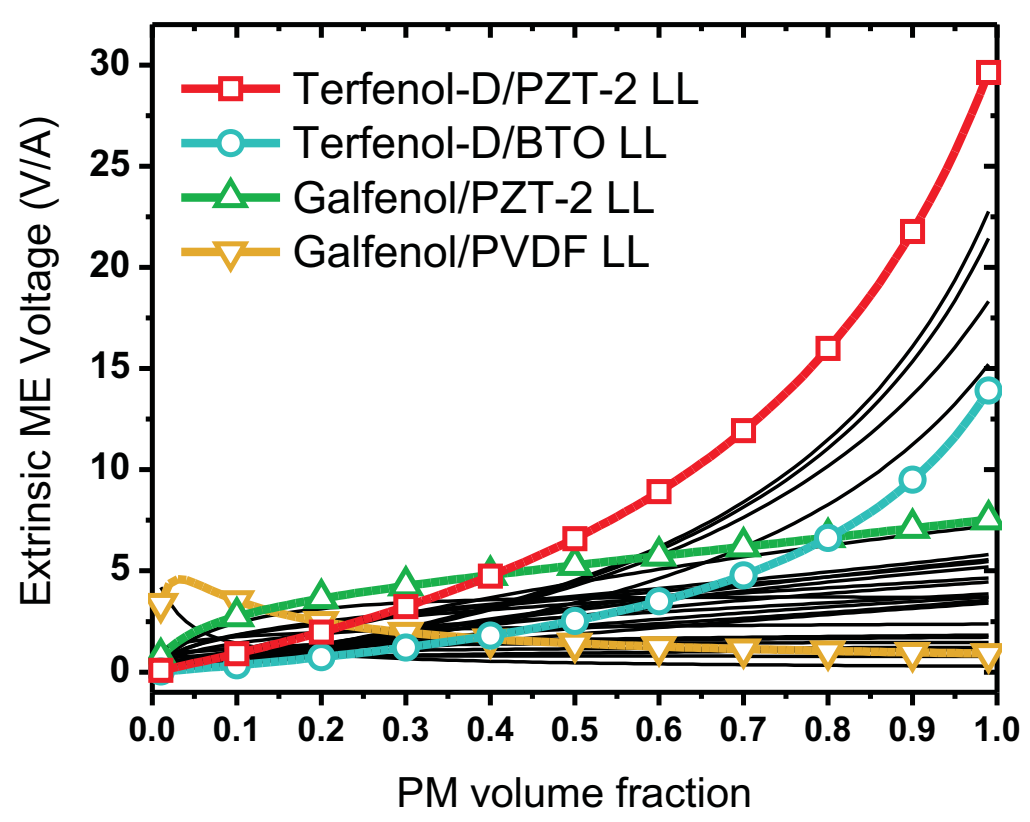

Figure 3.11: Extrinsic ME voltage coefficient for all the material combinations for an aspect ratio of $r=10$. Reproduced from [78] with permission.

has the highest $\hat{\alpha}$.

The PM volume fraction used in Figure 3.12 is the optimum for each composite and aspect ratio, which in some cases has to be reduced from the optimum of $\chi=1$ to a vales retaining $70 \%$ of the intrinsic ME coupling factor. In the case of Terfenol-D/PZT-2 and Galfenol/PZT-2 in the LL configuration, the optimum PM volume fraction is $\chi=1$, so they are reduced to 95 and $89 \%$, respectively.

For Metglas/PZT-2 and Metglas/PVDF in LL configuration, the optimum PM volume fraction changes with aspect ratio, as it can be seen in Figure 3.13. In the case of Metglas/PVDF for aspect ratios larger than 35000, the optimum PM volume fraction is given by the $70 \%$ retention criterion. When $r<35000$ is used, the optimum PM volume fraction decreases. The intrinsic ME voltage coefficient does not change when different aspect ratios are used but the demagnetizing factor does. For smaller PM volume fraction, the demagnetizing factor decreases because the thickness of the PM lamina used in (3.16) decreases. 


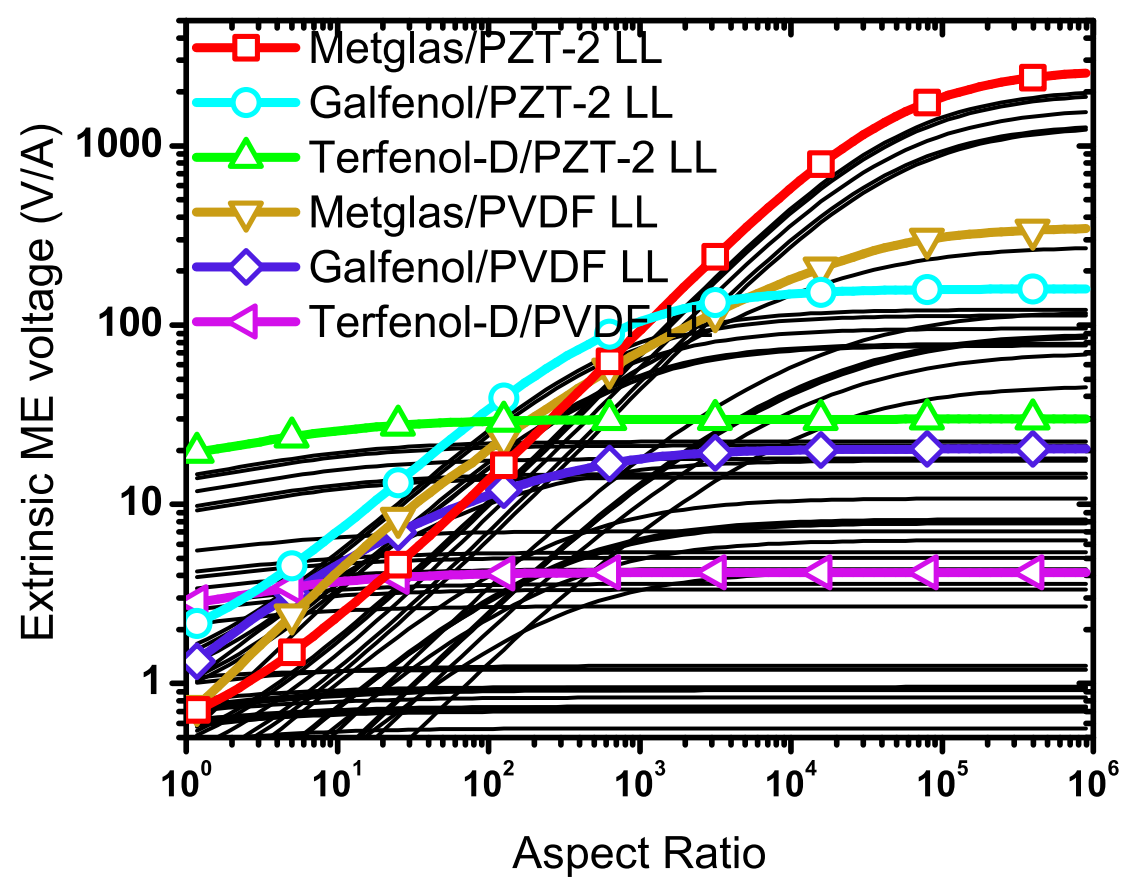

Figure 3.12: Extrinsic ME voltage coefficient for all the ME composite proposed as a function of the device aspect ratio. The optimum PM volume fraction for each material combination can be seen in Figure 3.13. Reproduced from [78] with permission. 
In some cases, like for Metglas/PVDF, the decrease in the demagnetizing factor (due to smaller optimum PM volume fraction required to stretch the PVDF) has a positive effect that more than compensates for the decrease in the intrinsic ME voltage resulting from using PVDF. This effect can be easily noticed for PVDF because PVDF is a soft PE material, not needing a thick PM lamina with high stiffness to strain it. A similar effect can be seen for Metglas/PZT-2 and for all the composites using Metglas. In Figure 3.13, for Metglas/PZT-2 LL, the aspect ratio $r$ decreases from right to left. For $30<r<500$, reducing $\chi$ reduces demagnetization more that enough to compensate for the reduction of intrinsic ME voltage coefficient $\alpha$ due to a lower $\chi$. However, For $r<30$, the effect of $\chi$ on $\alpha$ overcomes the reduction due to demagnetization, so the optimum goes back to $\chi=92 \%$. This behavior can be seen when using Metglas, but not on Terfenol-D and Galfenol, because the high $\mu^{P M}$ of Metglas, which makes the composite very susceptible to demagnetization, unless $\chi$ is so low that demagnetization is no longer relevant, and the performance is again controlled by the effect of $\chi$ on $\alpha$, i.e., the loss of magnetostriction.

The LL configuration yields maximum extrinsic ME voltage. However, LL configuration requires an insulating layer to prevent current leakage, thus fabrication is more complex. For this reason the LT configuration (most commonly used in the literature) is studied independently from the LL configuration. The predictions are shown in Figure 3.14.

LT Metglas/PVDF has the highest intrinsic voltage, as it is discussed in Section 3.3.2. Metglas/PVDF is the best option when aspect ratios higher than 250 are used. When aspect ratios from 4 to 250 are used, Galfenol/PVDF has the highest extrinsic ME voltage, and for aspect ratios smaller than 4, Terfenol-D/PVDF is the best candidate. PVDF is the best PE candidate because it has high compliance, requiring less PM thickness to stretch it, thus resulting in a thicker PE, which effectively increases the electric field. Note that, since the PM volume fraction for the composites with PVDF are small, resulting in a thin PM layer, the demagnetizing effect has a smaller impact than for other composites.

As it can be seen in Figure 3.15, the optimum PM volume fraction for LT Metglas/PVDF 


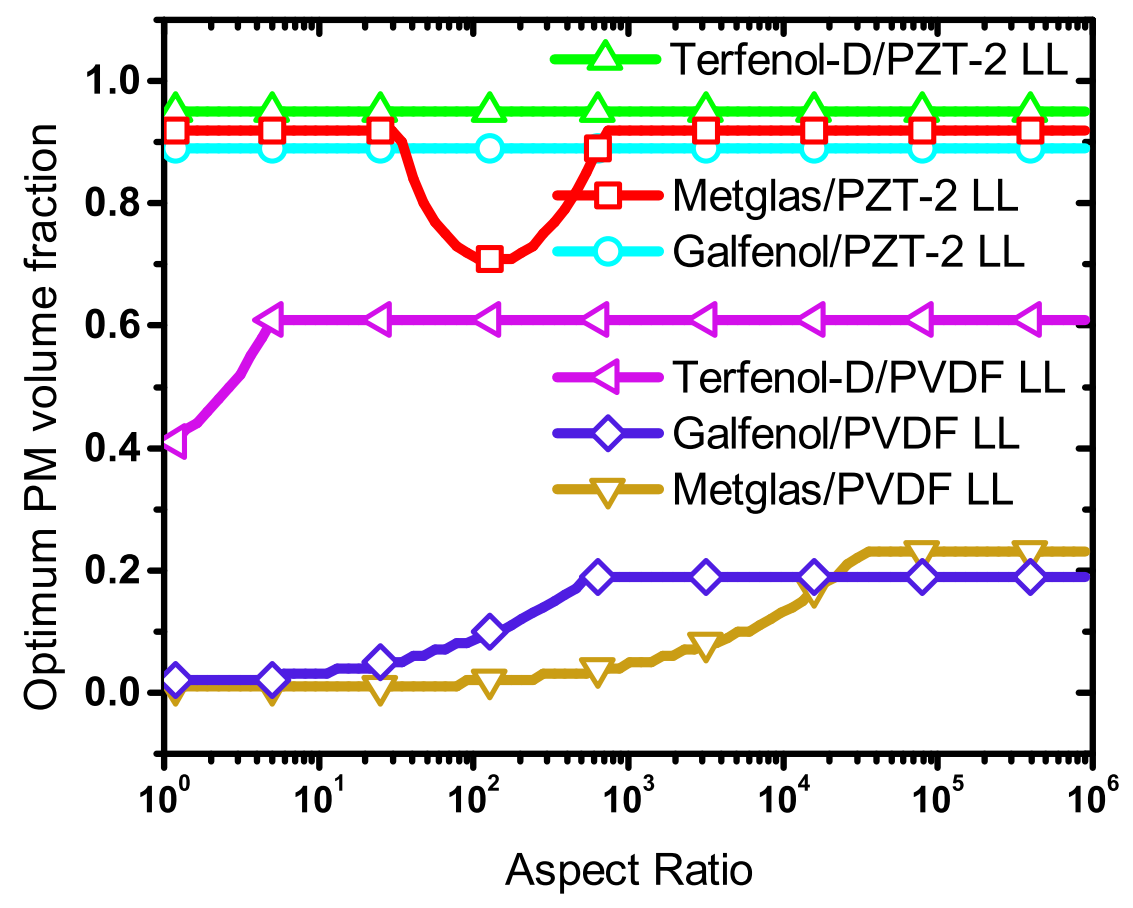

Figure 3.13: Optimum PM volume fraction, to maximize $\hat{\alpha}$, of Terfenol-D/PZT-2, Galfenol/PZT-2, Metglas/PZT-2, and Metglas/PVDF in LL configuration as a function of the device aspect ratio. Reproduced from [78] with permission. 


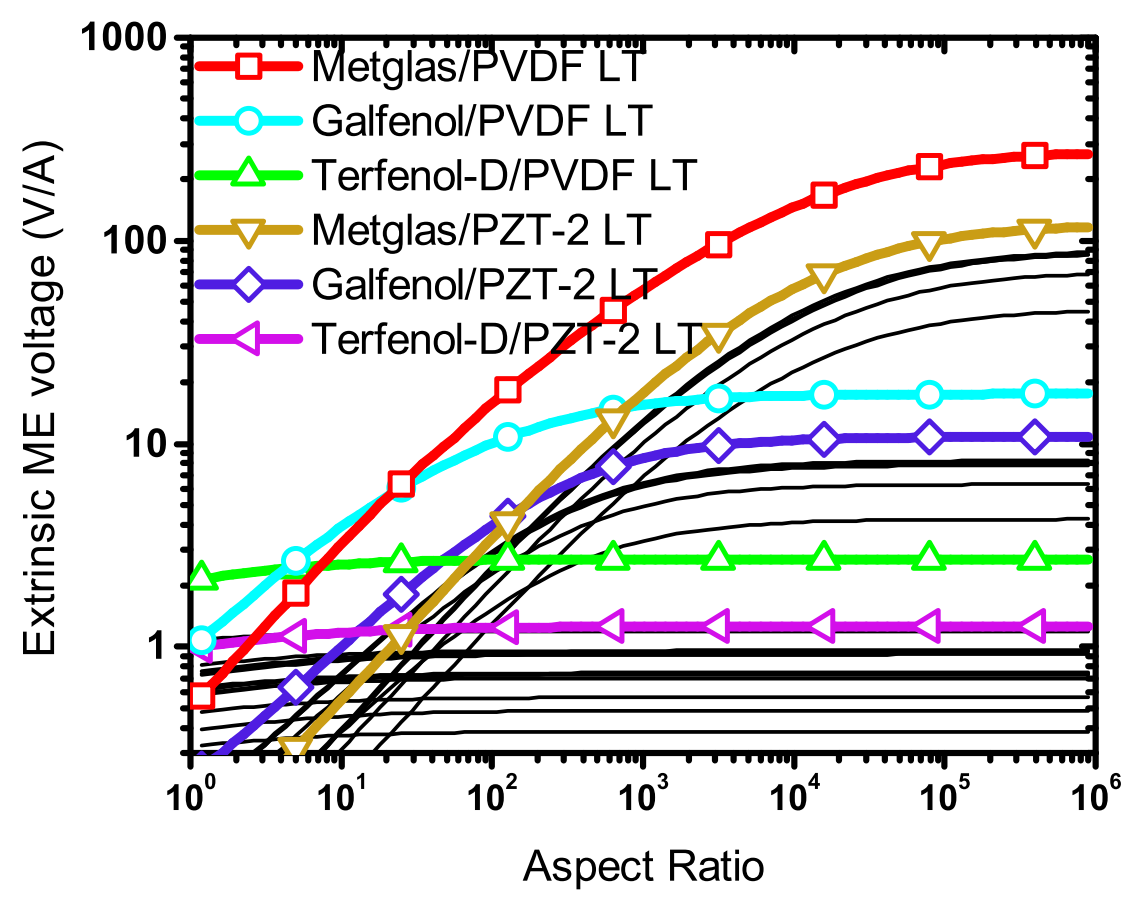

Figure 3.14: Extrinsic ME voltage coefficient all the ME composites in LT configuration as a function of the device aspect ratio. The optimum PM volume fraction for each configuration can be seen in Figure 3.15 . 
composite increases when aspect ratio increases. The optimum PM volume fraction for the intrinsic case (represented in the plot by $r=10^{6}$ ) is $23 \%$. When aspect ratios smaller that 300 are used, the optimum PM volume fraction is $1 \%$. This is due to the fact that smaller PM volume fraction results in smaller demagnetizing factor because the thickness of the PM phase is smaller. Furthermore, the PM phase does not need to have high stiffness because PVDF has a high compliance.

For LT Metglas/PVDF composite with $\chi \rightarrow 1 \%$, the intrinsic ME coupling factor plateaus at $75 \%$ of the highest ME coupling factor achievable. Thus, it satisfies the $70 \%$ retention criterion even at $\chi \rightarrow 1 \%$. If a PE phase with a lower compliance is used, such as in Metglas/PZT-2 composite, the optimum PM volume fraction does not decrease as much as for Metglas/PVDF because a thicker PM phase is required to drive the relatively stiff PZT-2.

A similar effect to the one shown by Metglas/PVDF can be seen with Galfenol/PVDF and Terfenol-D/PVDF, but in these composites the decrease in optimum PM volume fraction with decreasing aspect ratio is less severe than for Metgals/PVDF due to smaller magnetic permeability of Galfenol and Terfenol-D. An additional advantage of using PVDF instead of PZT materials is that PVDF is lead-free.

\subsubsection{Extrinsic ME charge coefficient}

Similarly to Section 3.4.1, the extrinsic ME charge coefficient $\hat{\beta}$ is defined as

$$
\left.\hat{\beta}=\frac{D}{H_{0}}\right]_{E=0}
$$

The intrinsic ME charge coefficient for the homogenized composite subject to magnetic field $H_{3}$ (see Section 3.4), is defined [55] as

$$
\left.\hat{\beta}=\frac{D}{H_{3}}\right]_{E=0}
$$




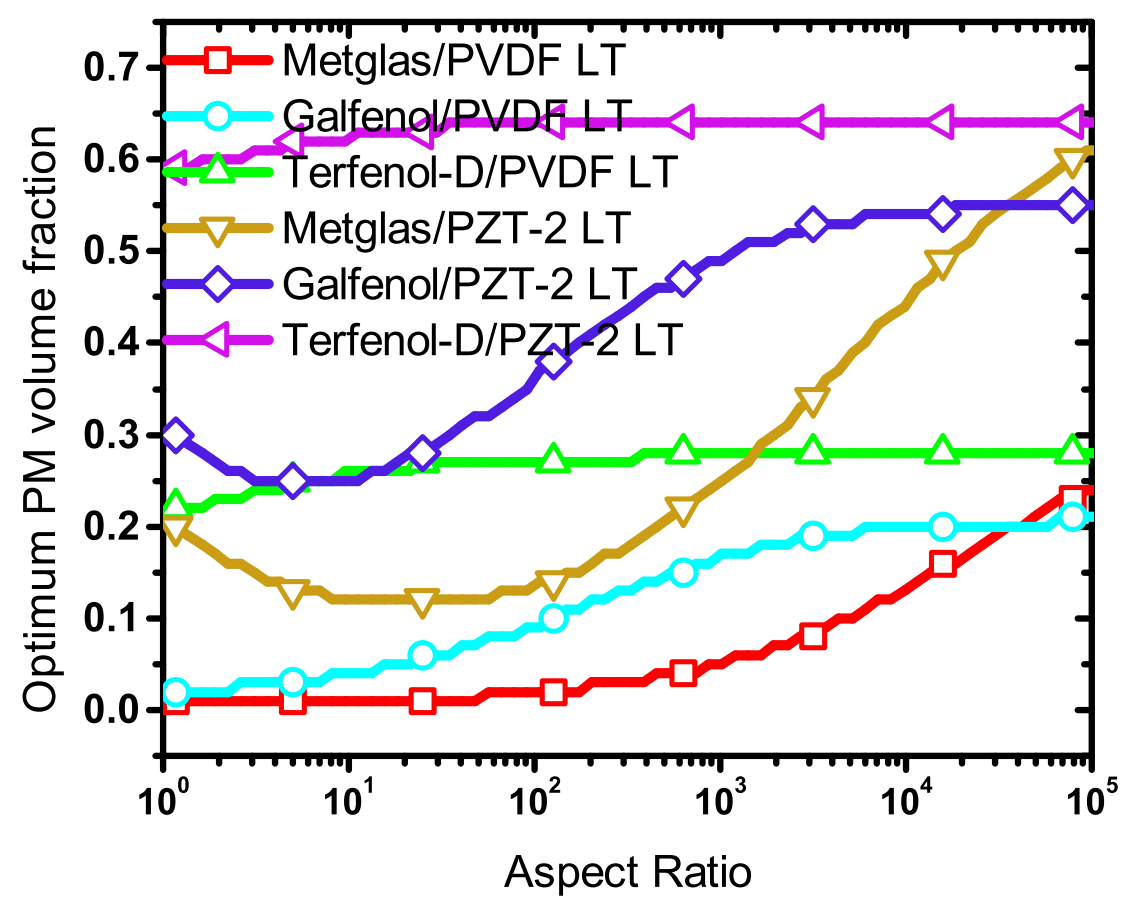

Figure 3.15: Optimum PM volume fraction (dimensionless) of Terfenol-D/PVDF, Galfenol/PVDF, Metglas/PVDF, Terfenol-D/PZT-2, Galfenol/PZT-2, and Metglas/PZT2 in LT configuration as a function of the device aspect ratio. Reproduced from [78] with permission. 
At this point is convenient to define the intrinsic ME charge coefficient for the homogenized composite subject to magnetic field $H_{3}^{\prime}$ (which is relevant in the magnetic phase only), as follows

$$
\left.\beta^{\prime}=\frac{D}{H_{3}^{\prime}}\right]_{E=0}
$$

which results in

$$
\begin{array}{r}
\left.\left.\frac{\hat{\beta}}{\beta^{\prime}}=\frac{D}{H_{0}} \times \frac{H_{3}^{\prime}}{D}\right]_{E=0}=\frac{H_{3}^{\prime}}{H_{0}}\right]_{E=0}= \\
\left.\frac{\hat{\beta}}{\beta^{\prime}}=\frac{\mu_{0}}{\mu_{0}+N_{3}\left(\mu^{\prime}-\mu_{0}\right)}\right]_{E=0}
\end{array}
$$

where $\beta^{\prime}$ and $\mu^{\prime}$ are defined in the same way as in Section 3.4.1. In the case of longitudinal magnetization $\beta=\beta^{\prime}$, but for transverse magnetization $\beta^{\prime}$ it has to be calculated using appropriate boundary conditions.

Also note that in (3.28), the magnetic permeability $\mu^{\prime}$ is the magnetic permeability of the PM phase when mechanically bonded to the PE phase, for a constant electric field $E=0$, i.e.,

$$
\left.\left.\mu^{\prime}=\frac{B_{3}^{\prime}}{H_{3}^{\prime}}\right]_{E=0} \frac{B^{P M}}{H^{P M}}\right]_{E=0}
$$

which must be calculated using the corresponding boundary conditions. The analytical expression is provided in the Website [69].

Predicted extrinsic ME charge coefficients for composites with aspect ratio $r=10$ are reported in Figure 3.16. Terfenol-D/PZT-5H in LT configuration has the best performance $\left(\hat{\beta}=77 \times 10^{-9} C A^{-1} m^{-1}\right.$ and $\kappa=0.08$ for $\left.\chi=95 \%\right)$. This is due to the smaller demagnetizing effect in a material, such as Terfenol, with relatively small magnetic permeability. In the LL configuration, the optimum extrinsic ME charge coefficient $\hat{\beta}=41 \times 10^{-9} \mathrm{CA}^{-1} \mathrm{~m}^{-1}$ is good as well, with the advantage of occurring at $\chi=55 \%$, which has a $\kappa=0.30$. Even Galfenol, 


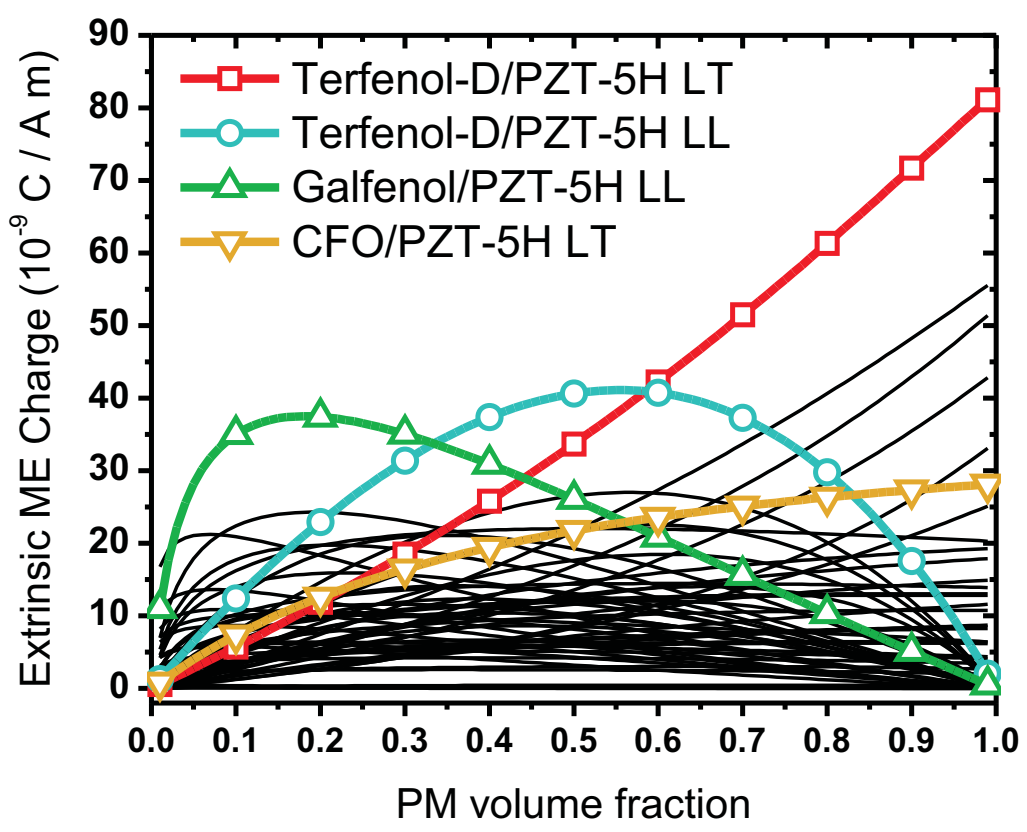

Figure 3.16: Extrinsic ME charge coefficient for all material combinations and aspect ratio $r=10$.

which has higher magnetic permeability than Terfenol, shows a high ME extrinsic charge coefficient of $\hat{\beta}=38 \times 10^{-9} C A^{-1} m^{-1}$, due to the higher intrinsic ME charge coefficient of Galfenol/PZT-5H in LL configuration. The later is just one example where intrinsic performance is useful to explain an extrinsic feature of a ME composite.

To evaluate the ME charge performance of the $28 \mathrm{ME}$ composites studied in this work, the extrinsic ME charge coefficient as a function of the aspect ratio is calculated and reported in Figure 3.17. When smaller aspect ratios are used, the demagnetizing effect is stronger for materials with high magnetic permeability, resulting in higher extrinsic ME charge coefficient for materials with smaller magnetic permeability. For aspect ratios $r>16000$, Metglas/PZT$5 \mathrm{H}$ in the LT configuration $(\chi=95 \%)$ holds the highest ME charge coefficient. For aspect ratios $126<r<16000$, Metglas/PZT-5H in the LL configuration $(\chi=10 \%$ for $r=126$ and $\chi=41 \%$ for $r=16000$ ) shows the highest $\hat{\beta}$. For aspect ratios $37<r<126$, Galfenol/PZT-5H in the LL configuration $(\chi=31 \%)$ shows the highest $\hat{\beta}$. For aspect ratios 


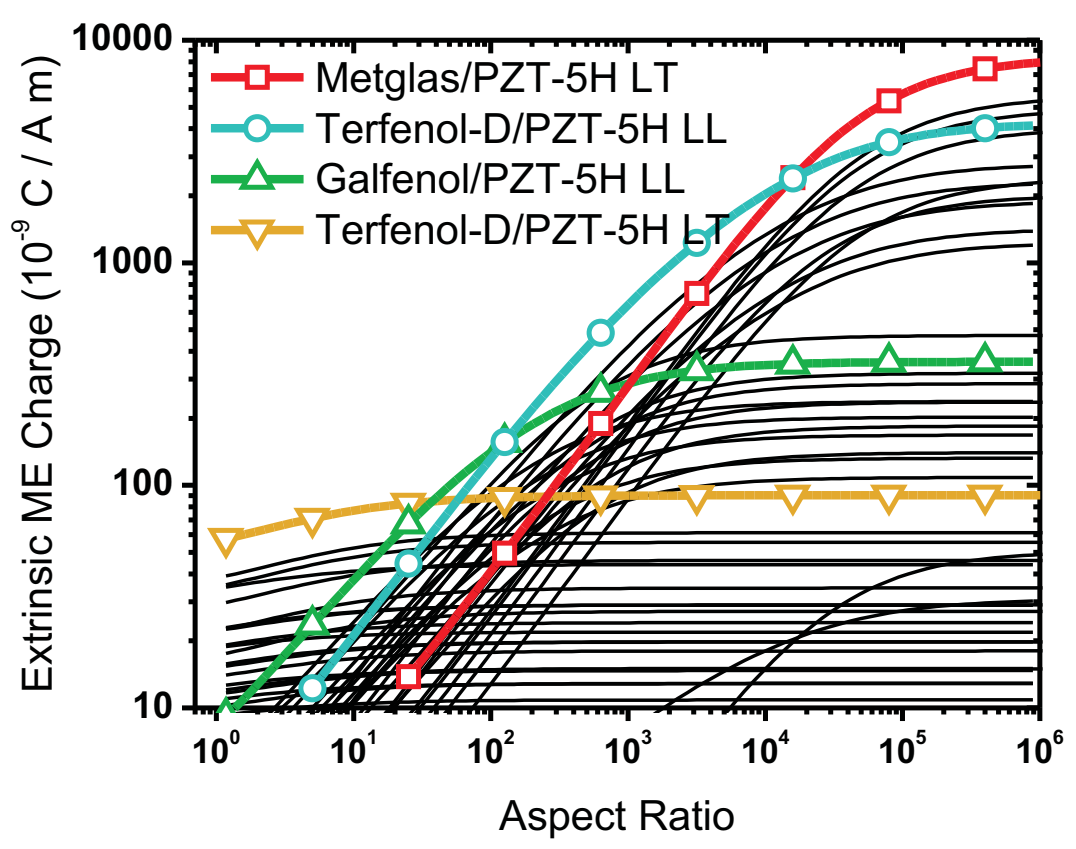

Figure 3.17: Extrinsic ME charge coefficient for all material combinations as a function of aspect ratio. Optimum PM volume fraction is used in LL configuration. $70 \%$ retention volume fraction is used for LT configuration.

$r<37$, Terfenol-D/PZT-5H in the LT configuration $(\chi=95 \%)$ shows the highest $\hat{\beta}$.

The optimum PM volume fraction for each configuration discussed in this Section varies similarly to Figure 3.13 but it is not shown for sake of space. Actual values can be calculated using the formulas provided Website [69].

\subsubsection{Extrinsic ME coupling factor}

The extrinsic ME coupling factor is defined similarly to the intrinsic ME coupling factor in Chapter 2, but using the externally applied, specific magnetic work. The later takes into account both the intrinsic work and the work in the surrounding medium (vacuum or air). Therefore,

$$
\hat{\kappa}^{2}=\frac{W_{E}^{G}}{\hat{W}_{M}^{A}}
$$


were $\hat{W}_{M}^{A}$ is the extrinsic magnetic work applied (specific magnetic work externally applied), when the electric field in the composite is constant. The extrinsic magnetic work applied can be obtained as follows

$$
\left.\left.\hat{W}_{M}^{A}=\frac{B_{0} \times H_{0}}{2}\right]_{E=0}=\frac{\mu_{e x t}\left(H_{0}\right)^{2}}{2}\right]_{E=0}
$$

were $H_{0}$ is the externally applied magnetic field, $B_{0}$ is the externally applied magnetic flux, and $\mu_{\text {ext }}$ is the magnetic permeability of the medium (vacuum, or air) with the composite structure inside. When the magnetic field is applied far enough (at infinity), the value of $\mu_{\text {ext }}$ approaches the magnetic permeability of vacuum $\mu_{0}$.

Because the average demagnetizing factor formula (3.16) only applies to a single phase lamina, in this work the PE phase is included in the surrounding medium and $\kappa^{\prime}$ is defined as

$$
\left(\kappa^{\prime}\right)^{2}=\frac{W_{E}^{G}}{W_{M}^{\prime A}}
$$

where the extrinsic work applied in terms of magnetic field $H_{3}^{\prime}$ in the PM phase is

$$
\left.\left.\left.W_{M}^{\prime A}=\frac{B_{3}^{\prime} \times H_{3}^{\prime}}{2}\right]_{E=0}=\frac{B^{P M} \times H^{P M}}{2}\right]_{E=0}=\frac{\mu^{\prime}\left(H_{3}^{\prime}\right)^{2}}{2}\right]_{E=0}
$$

and $\kappa^{\prime}$ has to be calculated for all four configurations. It can be shown that:

$$
\left(\kappa_{i j}^{\prime}\right)^{2}=\frac{\left(\beta_{i j}^{\prime}\right)^{2}}{\epsilon_{j j}^{H} \times \mu_{i i}^{\prime E}}
$$

were $\epsilon^{H}$ is the dielectric constant of the composite at constant magnetic field, calculated in 
Chapter 2. Next, the extrinsic ME coupling factor can be obtained as follows

$$
\begin{array}{r}
\left.\left.\left(\frac{\hat{\kappa}}{\kappa^{\prime}}\right)^{2}=\frac{W_{E}^{G}}{\hat{W}_{M}^{A}} \times \frac{W_{M}^{\prime A}}{W_{E}^{G}}=\frac{W_{M}^{\prime A}}{\hat{W}_{M}^{A}}=\frac{H_{3}^{\prime}}{H_{0}} \times \frac{B_{3}^{\prime}}{B_{0}}\right]_{E=0}=\left(\frac{H_{3}^{\prime}}{H_{0}}\right)^{2} \times \frac{\mu^{\prime}}{\mu_{0}}\right]_{E=0} \\
\left.\left(\frac{\hat{\kappa}}{\kappa^{\prime}}\right)^{2}=\frac{\mu^{\prime} \mu_{0}}{\left(\mu_{0}+N_{3}\left(\mu^{\prime}-\mu_{0}\right)\right)^{2}}\right]_{E=0}
\end{array}
$$

Note that $\mu^{\prime}$ is the magnetic permeability of the PM phase, bonded to the PE phase, when the electric field is constant, which has to be calculated.

For aspect ratios $r \rightarrow \infty$ the demagnetizing factor $N_{3} \rightarrow 0$ reducing (3.35) to

$$
\hat{\kappa}=\kappa^{\prime} \sqrt{\left.\frac{\mu^{\prime}}{\mu_{0}}\right]_{E=0}}
$$

This equation implies that $\hat{\kappa}>\kappa^{\prime}$ because $\mu^{\prime}>\mu_{0}$ when a PM material of any kind is present in the medium (air or vacuum). Values of $\hat{\kappa}$ could be higher than unity because (3.30) is a ratio of specific work over different volumes, with $W_{E}^{G}$ measured over the volume of the device and $\hat{W}_{M}^{A}$ over the volume where the external magnetic field is applied.

Maximum values of extrinsic ME coupling coefficient (obtained at optimum PM volume fraction for all materials) are shown in Figure 3.18. It can be seen that Metglas/PZT-5H in LL configuration has the highest ME coupling coefficient for aspect ratios higher than 350. This is due to the fact that Metglas and PZT-5H have the maximum PM and PE coupling factors, respectively. For aspect ratios between 27 and 350 Galfenol/PZT-5H in the LL configuration has the highest extrinsic ME coupling coefficient. As previously discussed, demagnetization is smaller on Galfenol than on Metglas. Finally, for aspect ratios smaller than 27, Terfenol-D/PZT-5H LL has the highest $\hat{\kappa}$.

\subsection{Conclusions}

The effects of demagnetization are drastic, not only in magnitude but also drastically changing material selection and volume fraction, both as function of the device's geometrical aspect 


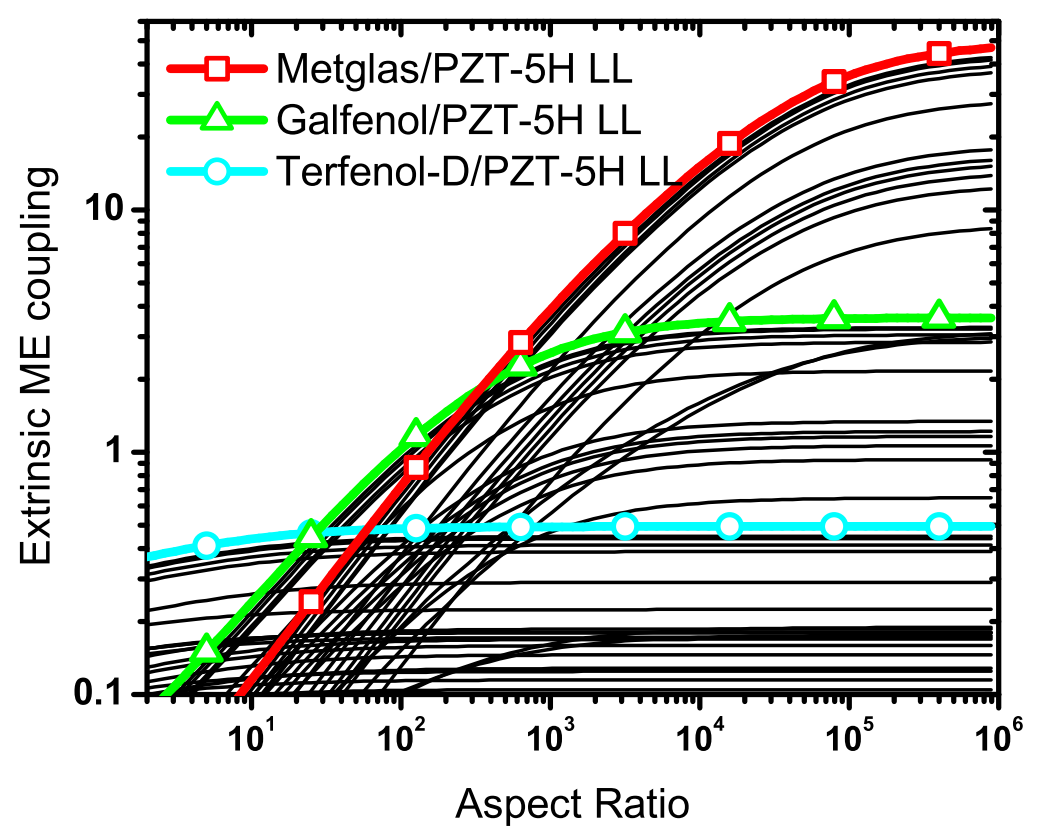

Figure 3.18: Optimum extrinsic ME coupling factor (dimensionless) for all material combinations as a function of aspect ratio.

ratio. PM materials with high magnetic permeability are very sensitive to demagnetizing effects and loose their comparative advantage even if they also posses high intrinsic coupling. There are two ways to minimize demagnetization: use a PM with low permeability $\mu$ or a PE with high compliance $S$. A composite with low PM permeability $\mu$ is better because the usable magnetic field $H_{3}$ in (3.15) is inversely proportional to $\mu$, so less $\mu$ means the performance is less affected by demagnetization. A composite with high PE compliance (e.g., PVDF) is best in LT and LL configurations because less PM is needed, and since only the PM is affected by demagnetization, the extrinsic performance is less affected.

The material combinations identified as having best intrinsic performance are replaced by different material combinations when extrinsic properties are calculated. Furthermore, the best material selection, optimum volume fraction, and even optimal configuration vary with the aspect ratio, thus requiring to include device geometry in the design process. The LL configuration yields maximum extrinsic ME voltage. For an aspect ratio of 10, ME 
devices based on Terfenol-D are predicted to have the highest extrinsic voltage coefficient due to its relatively small magnetic permeability $\mu_{33} / \mu_{0}=3$ (Table 3.2). In some cases, like for Metglas/PVDF, the decrease in the demagnetizing factor (due to smaller optimum PM volume fraction required to stretch the compliant PVDF) has a positive effect that more than compensates for the decrease in the intrinsic ME voltage resulting from using PVDF. However, different PM phases are required to achieve optimum performance at different values of aspect ratio.

The highest extrinsic $\alpha$ and $\kappa$ are predicted when the ME composite is polarized and magnetized in the longitudinal direction, justifying the more complicated fabrication required by longitudinal polarization, which requires an insulator to prevent charge leakage from the PE phase through the PM phase. Longitudinal magnetization yields higher extrinsic open circuit sensitivity due to the higher strain produced. 


\section{Chapter 4}

\section{Prediction of magnetoelectric}

\section{coefficients for particulate composites}

In the present work magnetoelectric (ME) properties of particulate composites are calculated using Eshelby theory and two homogenization techniques: dilute approximation and MoriTanaka mean field theory ${ }^{1}$. A method that allows the calculation of all ME properties under any boundary conditions is proposed. These boundary conditions are dictated by the experimental configuration, e.g. films on a substrate, free-standing composites, etc. Predictions are compared with calculations reported by Harshe et al. and Nan et al., and good correlation is obtained with those, but to achieve good correlation with experimental data, the conductivity of the piezomagnetic (PM) phase must be taken into account, and a method is proposed to that effect. Percolated composites do not have any piezoelectric (PE) or ME properties because the charge leaks through the conductive PM phase. The experimental parameters that influence the percolation threshold are discussed and the best particulate composite design is proposed. Unlike previous models that did not account for conductivity, correlation between the proposed model and experimental data is much better.

\footnotetext{
${ }^{1}$ This chapter was submitted to Smart Materials and Structures journal
} 


\subsection{Introduction}

ME composites are made of piezoelectric and magnetostrictive phases coupled by a strain field. The composite geometry can vary from laminated to particulate. The particulate composites do not need to experience overall strain to produce ME effect. While particulate composites deposited on a rigid substrate are able to produce ME effect, laminated composites do not, due to the clamping effect. Percolation of the conductive dispersed phase is a non desired effect in ME composites because it leaks the generated charge. Taking into account the dispersed phase geometry (e.g. spherical, rods, plates, etc.) and phase size, a design criterion will be established in $\S 4.4$ to prevent percolation even when a high amount of PM phase is used.

Several models for particulate composites had been proposed in literature [46, 79, 42, 80, $47,81,82]$. The geometry used for the dispersed phase include spheres, elliptic cylinder, circular cylinder, disk, and ribbons. Literature predictions deviate from experimental data because the conductive behavior of the PM phase has not been considered.

After the conductive PM phase percolates on a ME composite, the ME and PE properties vanish because the charge leaks through the conductive path of the percolated PM phase $[83,84]$. The experimental parameters that influence percolation threshold as well as the effect of the dispersed phase geometry are considered in this work. Spherical particles are selected for because they maximize the percolation threshold. The actual percolation threshold is not calculated because it depends on experimental parameters, but guidelines on how to increase it are given, which result in better ME properties.

Before the conductive PM phase percolates, the ME properties of the composite are affected by the conductivity of the PM phase. Previously, the conductive behavior of the PM phase was ignored, leading to poor correlation with experimental data $[79,47]$. This is due to the fact that the constitutive equations used are static and do not consider conductivity. If the material is a conductor instead of an insulator, the electric field inside will decrease to zero after a short period of time. To take this into account the model would have to consider the 
conductivity and the model would change from static to dynamic, increasing the complexity and adding more variables and constants. In this work the conductive behavior of the PM phase is taken into account using the approach proposed in Chapter 2 and Chapter 3 for laminate structures. As a result of it, the model predicts ME properties substantially closer to the experimentally measured values.

\subsection{Model}

The constitutive equations used in this work consider the elastic, electric, magnetic, piezoelectric, piezomagnetic, and magnetoelectric interaction. The polarization and magnetization are in the 3-direction for the PM and PE phase, resulting in transversally isotropic symmetry around the 3-direction. The linear constitutive equations are:

$$
\left[\begin{array}{l}
\sigma_{1} \\
\sigma_{2} \\
\sigma_{3} \\
\sigma_{4} \\
\sigma_{5} \\
\sigma_{6} \\
D_{1} \\
D_{2} \\
D_{3} \\
B_{1} \\
B_{2} \\
B_{3}
\end{array}\right]=\left[\begin{array}{cccccccccccc}
C_{11} & C_{12} & C_{23} & 0 & 0 & 0 & 0 & 0 & e_{31} & 0 & 0 & q_{31} \\
C_{12} & C_{11} & C_{23} & 0 & 0 & 0 & 0 & 0 & e_{31} & 0 & 0 & q_{31} \\
C_{23} & C_{23} & C_{33} & 0 & 0 & 0 & 0 & 0 & e_{33} & 0 & 0 & q_{33} \\
0 & 0 & 0 & C_{44} & 0 & 0 & 0 & e_{15} & 0 & 0 & q_{15} & 0 \\
0 & 0 & 0 & 0 & C_{44} & 0 & e_{15} & 0 & 0 & q_{15} & 0 & 0 \\
0 & 0 & 0 & 0 & 0 & C_{66} & 0 & 0 & 0 & 0 & 0 & 0 \\
0 & 0 & 0 & 0 & e_{15} & 0 & \epsilon_{11} & 0 & 0 & \lambda_{11} & 0 & 0 \\
0 & 0 & 0 & e_{15} & 0 & 0 & 0 & \epsilon_{11} & 0 & 0 & \lambda_{11} & 0 \\
e_{31} & e_{31} & e_{33} & 0 & 0 & 0 & 0 & 0 & \epsilon_{33} & 0 & 0 & \lambda_{33} \\
0 & 0 & 0 & 0 & q_{15} & 0 & \lambda_{11} & 0 & 0 & \mu_{11} & 0 & 0 \\
0 & 0 & 0 & q_{15} & 0 & 0 & 0 & \lambda_{11} & 0 & 0 & \mu_{11} & 0 \\
q_{31} & q_{31} & q_{33} & 0 & 0 & 0 & 0 & 0 & \lambda_{33} & 0 & 0 & \mu_{33}
\end{array}\right] *\left[\begin{array}{l}
s_{1} \\
s_{2} \\
s_{3} \\
s_{4} \\
s_{5} \\
s_{6} \\
E_{1} \\
E_{2} \\
E_{3} \\
H_{1} \\
H_{2} \\
H_{3}
\end{array}\right]
$$

where $C$ is the elastic tensor measured at constant electric and magnetic field $\left(E_{i}=0\right.$ and $\left.H_{i}=0\right), \epsilon$ is the dielectric tensor measured at constant strain and magnetic field $\left(s_{i}=0\right.$ and $\left.H_{i}=0\right), \mu$ is the magnetic permeability tensor measured at constant strain and constant electric field, $e$ is the piezoelectric tensor defined as:

$$
\left.e_{i j}=\frac{\sigma_{j}}{E_{i}}\right]_{s=0, H=0}
$$

$q$ is the piezomagnetic tensor defined as:

$$
\left.q_{i j}=\frac{\sigma_{j}}{H_{i}}\right]_{s=0, E=0}
$$


and $\lambda$ is the magnetoelectric tensor defined as:

$$
\left.\lambda_{i i}=\frac{D_{i}}{H_{i}}\right]_{s=0, E=0}
$$

Equation (4.1) can be written as:

$$
\Sigma_{i J}=L_{i J M n} Z_{M n}
$$

where $\Sigma_{i J}$ contains $\sigma, D$ and $B$, and $Z_{M n}$ contains the $s, E$, and $H$, and $L_{i J M n}$ contains the materials properties. The index mapping is made using an expanded Voigt notation:

$$
\begin{aligned}
& 11 \rightarrow 1, \quad 22 \rightarrow 2, \quad 33 \rightarrow 3, \quad 23 \rightarrow 4, \quad 31 \rightarrow 5, \quad 12 \rightarrow 6, \\
& 41 \rightarrow 7, \quad 42 \rightarrow 8, \quad 43 \rightarrow 9, \quad 51 \rightarrow 10, \quad 52 \rightarrow 11, \quad 53 \rightarrow 12 .
\end{aligned}
$$

As it can be noticed, some indexes are capitalized and other are not. In this work the capitalized indexes have values from 1 to 5 and the non capitalized indexes have values from 1 to 3 . The indexes that have values from 1 to 5 indicates the physics, i.e., 4 is electric, 5 is magnetic and 1,2, and 3 are for mechanics. The indexes that range from 1 to 3 relate to the direction. For example, 41 would be electric in the 1-direction.

The proposed model is based on Eshelby theory [85], that allows us to calculate the elastic, magnetic, and electric field inside an ellipsoid when the composite is subject to an elastic, magnetic, and/or electric perturbation. After the Eshelby tensor is obtained, an approximation can be used to calculate the homogenized properties of the composite. Eshelby theory was first introduced for elastic materials only, but the same theory can be used for any linear constitutive model. The Eshelby tensor is defined as follows:

$$
Z_{M n}=S_{M n A b} Z_{A b}^{*}
$$


where $Z_{A b}^{*}$ is the eingenfield, which is the field (elastic, magnetic, and electric) in the ellipsoid, due to an external perturbation, when the inclusion is removed from the matrix. For example, the elastic strain produced in a PM particle when a magnetic field is applied to the particle in vacuum. Next, $Z_{M n}$ is the field inside the particle when the particle with an eingenfield is introduced in the matrix. For example, the strain produced in a PM particle embedded in a matrix when a magnetic field is applied. Finally, $S_{M n A b}$ is the Eshelby tensor.

The Eshelby tensor can be calculated using the Green function for the model described by (4.1) and the properties of the matrix $\left(L_{i J m n}^{m}\right)^{2}$, as follows [79]:

$$
\begin{aligned}
S_{m n a b} & =\frac{1}{8 \pi}\left[C_{i j a b}^{m}\left(G_{m j i n}+G_{n j i m}\right)+q_{i a b}^{m}\left(G_{m 5 i n}+G_{n 5 i m}\right)\right] \\
S_{m n 4 b} & =\frac{1}{8 \pi} \epsilon_{i b}^{m}\left(G_{m 4 i n}+G_{n 4 i m}\right) \\
S_{m n 5 b} & =\frac{1}{8 \pi}\left[q_{b i j}^{m}\left(G_{m j i n}+G_{n j i m}\right)+\mu_{i b}^{m}\left(G_{m 5 i n}+G_{n 5 i m}\right)\right] \\
S_{4 n a b} & =\frac{1}{4 \pi}\left(C_{i j a b}^{m} G_{4 j i n}+q_{i a b}^{m} G_{45 i n}\right) \\
S_{4 n 4 b} & =\frac{1}{4 \pi} \epsilon_{i b}^{m} G_{44 i n} \\
S_{4 n 5 b} & =\frac{1}{4 \pi}\left(q_{b i j}^{m} G_{4 j i n}+\mu_{i b}^{m} G_{45 i n}\right) \\
S_{5 n a b} & =\frac{1}{4 \pi}\left(C_{i j a b}^{m} G_{5 j i n}+q_{i a b}^{m} G_{55 i n}\right) \\
S_{5 n 4 b} & =\frac{1}{4 \pi} \epsilon_{i b}^{m} G_{54 i n} \\
S_{5 n 5 b} & =\frac{1}{4 \pi}\left(q_{b i j}^{m} G_{5 j i n}+\mu_{i b}^{m} G_{55 i n}\right)
\end{aligned}
$$

The Green function is calculated as the integral over the volume of the inclusion (Figure 4.1):

$$
G_{M J i n}=\int_{-1}^{1} \int_{0}^{2 \pi} N_{M J}(\xi) D^{-1}(\xi) \xi_{i} \xi_{n} d \theta d \varsigma_{3}
$$

where $N_{M J}(\xi)$ and $D(\xi)$ are the cofactor and determinant of the $\left(L_{i J M n}^{m} \xi_{i} \xi_{n}\right)$ matrix, respec-

\footnotetext{
${ }^{2}$ Using superscript ${ }^{m}$ indicates properties of the matrix and ${ }^{d}$ indicates properties of the dispersed phase (particles).
} 
tively.

The relationship between $\theta, \varsigma_{3}$, and $\xi_{n}$ are given by the dispersed phase geometry. First, from the $x_{n}$ Cartesian coordinate system the following mapping is made, $\xi_{n}=x_{n} / a_{n}$, where $a_{n}$ are the semi-principal axes of the ellipsoid. The particle domain ( $\Omega$ in Figure 4.1) is defined as:

$$
\frac{x_{1}^{2}}{a_{1}^{2}}+\frac{x_{2}^{2}}{a_{2}^{2}}+\frac{x_{3}^{2}}{a_{3}^{2}}<1
$$

In this work, spherical particles are considered $\left(a=a_{1}=a_{2}=a_{3}\right)$ because spherical particles increase the percolation threshold. This will be further discussed in Section 4.4. The mapping of the spherical inclusion surface is defined as a function of $\theta$ and $\varsigma_{3}$ as follows:

$$
\begin{aligned}
& \xi_{1}=\sqrt{1-\varsigma_{3}^{2}} * \cos [\theta] \\
& \xi_{2}=\sqrt{1-\varsigma_{3}^{2}} * \sin [\theta] \\
& \xi_{3}=\varsigma_{3}
\end{aligned}
$$

where $\theta$ is the angle around $x_{3}$ in Figure 4.1. Note that $x_{3}$ is the axis af transverse isotropy for both materials, as reflected in (4.1), but one could choose different polarization and magnetization directions. Using polarization and magnetization perpendicular to each other results in different ME response, similarly to the TL (or LT) configuration in laminated ME composites.

After the Eshelby tensor is obtained, the homogenized properties can be obtained by using a number of homogenization approximations, including the dilute approximation and Mori-Tanaka mean field theory. These approximations require that the size of the dispersed phase be much smaller than the size of the composite. In the case of the dilute approximation, no interaction between particles is taken into account. Due to this approximation, the dilute theory is accurate for small particulate volume fractions $\chi$ only. The homogenized properties 


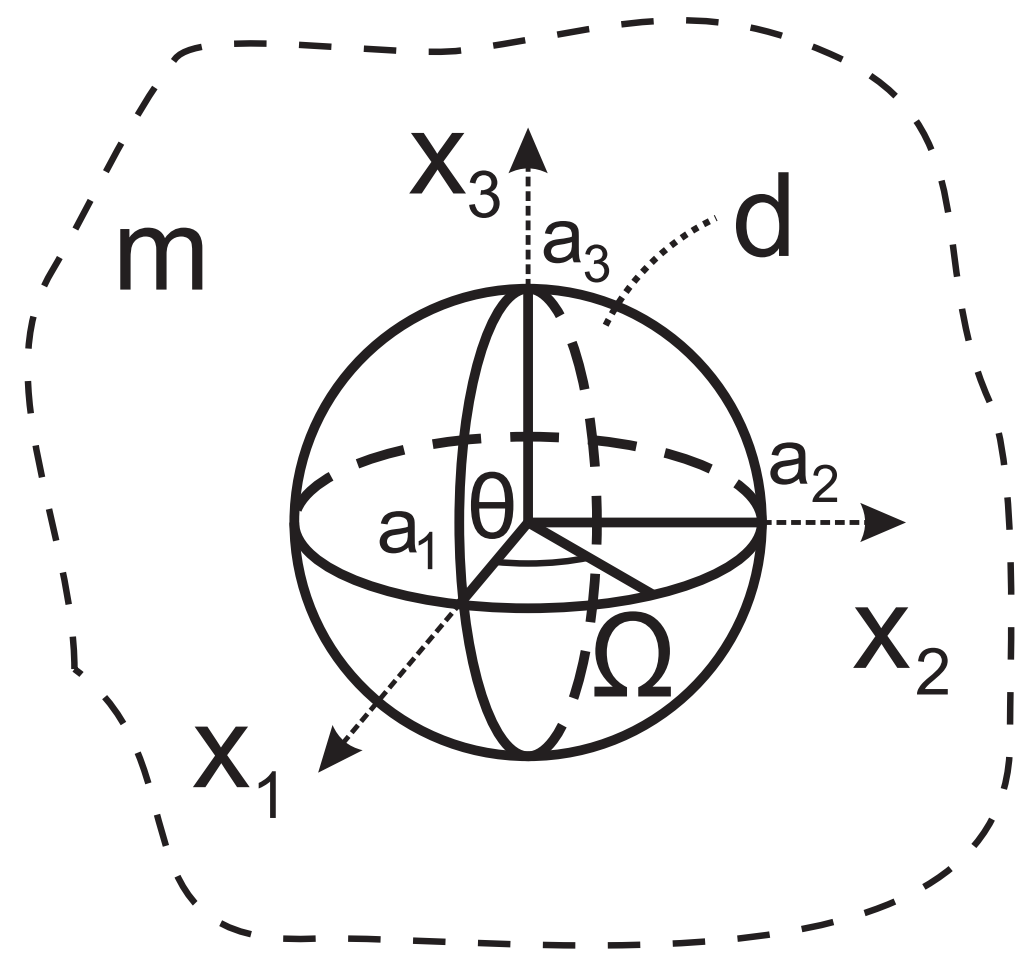

Figure 4.1: Geometry used in this model. Spherical dispersed phase in a matrix.

of the composite $\bar{L}_{i J M n}$ is obtained using dilute approximation [86] as follows:

$$
\bar{L}_{i J M n}=L_{i J M n}^{m}+\chi\left(L_{i J A b}^{d}-L_{i J A b}^{m}\right) V_{A b M n}^{-1}
$$

where $V_{A b M n}^{-1}$ is the inverse of $V_{M n A b}$ defined by ${ }^{3}$ :

$$
V_{M n A b}=I_{M n A b}+S_{M n J i}\left(L_{q R J i}^{m}\right)^{-1}\left(L_{q R A b}^{d}-L_{q R A b}^{m}\right)
$$

where $I_{M n A b}$ is the identity tensor:

$$
I_{M n A b}=\left[\begin{array}{l}
\left(\delta_{m a} \delta_{n b}+\delta_{m b} \delta_{n a}\right) / 2, \quad M, A \leq 3 \\
\delta_{n b}, \quad M=A=4 \quad \text { or } \quad M=A=5 \\
0, \quad \text { otherwise. }
\end{array}\right.
$$

Another method to calculate the homogenized properties of the composite is the Mori-

\footnotetext{
${ }^{3}$ The tensor is inverted using the approach discussed in Appendix A.5 in [87]
} 
Tanaka mean field theory [88], where the interaction between particles is taken into account making it a better method than the dilute approximation for higher values of $\chi$. The interaction between the particles is incorporated by using the volume averaged fields[86]. The homogenized properties of the composite are obtained as follows:

$$
\bar{L}_{i J M n}=L_{i J M n}^{m}+\chi L_{i J A b}^{m} T_{A b q R}^{-1}\left(L_{q R M n}^{d}-L_{q R M n}^{m}\right)
$$

where $T_{A b i J}^{-1}$ is the inverse of $T_{i J A b}$ :

$$
T_{i J A b}=(1-\chi)\left(L_{i J M n}^{d}-L_{i J M n}^{m}\right) S_{M n A b}+L_{i J A b}^{m}
$$

\subsection{Magnetoelectric constants}

After the homogenized properties tensor $\bar{L}_{i J M n}$ is obtained, the desired ME coefficient of coupling has to be calculated. The ME voltage coefficient is defined as:

$$
\left.\alpha_{33}=\frac{E_{3}}{H_{3}}\right]_{D_{3}=0}
$$

This can be obtained by solving the equation system given by (4.1) subject to appropriate boundary conditions. The boundary condition $D_{3}=0$ is given by the definition of $\alpha$. Since the electric field is measured in the 3-direction and the electrodes short circuit the 2- and 1-direction, then $E_{1}=E_{2}=0$. Since the magnetic field is applied only in the 3-direction, then $H_{1}=0$ and $H_{2}=0$. Finally, the mechanical boundary conditions have to be applied. In this work two different conditions are considered. The first condition is unrestrained meaning that the composite can move freely, then $\sigma_{M}=0$ on the whole boundary. The other condition is completely clamped, meaning that is restrained from displacement in all directions. The mechanical boundary conditions needed to prevent displacement are obtained by setting the strain is to zero in all directions $s_{M}=0$. Even though this configuration is not 
found in experiments, it is commonly used by other authors in theoretical calculations and will be useful for validation of the proposed model [47]. The method proposed to calculate the ME voltage coefficient allows us to take into account the real boundary conditions as well. For example, films deposited on a substrate that have mechanical boundary conditions $s_{1}=s_{2}=\sigma_{3}=0$ can be analyzed.

The ME charge coefficient is defined as:

$$
\left.\beta_{33}=\frac{D_{3}}{H_{3}}\right]_{E_{3}=0}
$$

Similarly to $\alpha, \beta$ can be obtained by solving the equation system given by (4.1) subject to appropriate boundary conditions. The boundary condition $E_{3}=0$ is given by the definition of $\beta$. Since the electric displacement is measured in the 3 -direction and the electrodes short circuit the 2- and 1-direction, then $E_{1}=E_{2}=0$. Since the magnetic field is applied only in the 3 -direction, then $H_{1}=0$ and $H_{2}=0$. Finally, the mechanical boundary conditions have to be applied. In this work for preliminary results only the unrestrained condition will be used.

The ME coupling factor is the ratio between the electrical work generated and magnetic work applied (or magnetic work generated and electrical work applied). It is an useful property to compare different ME composites in their ability to be used as energy transducers. It can be calculated as a function of other properties of the ME composite as follows [55]:

$$
\kappa_{33}^{2}=\frac{\beta_{33}^{2}}{\epsilon_{33}^{H} \mu_{33}^{E}}
$$

were $\epsilon_{33}^{H}$ is the dielectric constant in the 3-direction with $H=0$ and $\mu_{33}^{E}$ is the magnetic permeability in the 3-direction with $E=0$. To calculate the $\mathrm{ME}$ coupling factor the mechanical boundary conditions have to be specified. Similarly to $\beta$, in this work only the unrestrained condition will be used.

The effect of the surrounding medium on the magnetic field has to be considered when 
the ME properties are calculated. This is accounted by the demagnetizing factor. The values calculated by Equation (4.16) (4.17) (4.18) are the intrinsic ME properties. These properties are measured when the magnetic field is applied in the boundaries of the composite (internal magnetic field). These properties are intrinsic in the sense that they do not depend on the geometry of the ME composite.

The extrinsic properties take into account a magnetic field that is applied far enough from the boundaries of the ME composite. For example, the extrinsic ME voltage coefficient is:

$$
\left.\alpha_{33}=\frac{E_{3}}{H_{0}}\right]_{D_{3}=0}
$$

where $H_{0}$ is the externally applied magnetic field (far from the composite). The relationship between $H_{0}$ and $H_{3}$ is given by the demagnetizing effect as follows:

$$
H_{3}=H_{0} \frac{\mu_{0}}{\mu_{0}+N_{3}\left(\mu-\mu_{0}\right)}
$$

where $\mu$ is the magnetic permeability of the $\mathrm{ME}$ composite and $N_{3}$ is the demagnetizing factor. The ME composite is assumed to be a continuum. This is a valid approximation when the size of the dispersed particles is significantly smaller than the size of the composite. In the later case the homogenized properties of the composites can be used. The average value of $N_{3}$ for a plate can be calculated using the expression given by [71]. In this work only the intrinsic properties of the ME composites are calculated.

\subsection{Percolation threshold}

The composites studied in this work are made of a matrix and a dispersed phase. The matrix is connected in all directions, meaning that one can start on any side of the composite and reach the opposite side on a path that does not leave the matrix phase. When small volume fractions are used, the dispersed phase is not connected and as a consequence a path 
connecting two sides without leaving the dispersed phase is not possible. At this point the dispersed phase is not percolated. If higher volume fraction of the dispersed phase is used, the particles will start producing connected chains of the dispersed phase. At high $\chi$ a path that connect the sides of the composites, without leaving the dispersed phase, will be possible and this composite is in the percolated state. The percolation threshold is defined as the value of $\chi$ that separates the non-percolated state from the percolated state.

In ME composites, percolation is not desired because of the high conductivity of the PM phase (Terfenol-D, Metglas, Galfenol, Ferrites) [78]. Whenever the PM phase percolates, the charge produced in the $\mathrm{PE}$ phase leaks through the conductive path produced by the percolated PM phase. A well know value for the percolation threshold is the Sher-Zallen invariant, approximately $16 \%$. This value is for a composite obtained with non-agglomerated spherical particles with similar size for both, the dispersed and matrix precursor. Also, the particles have to have a size bigger than a $\mu m$, to prevent tunneling. Practically, the percolation threshold will be different than the Sher-Zallen invariant due to the dependence with the microstructure and it will be affected by the fabrication process used.

One of the main factors that influence the percolation threshold is the geometry of the dispersed phase. For example, if very long fibers are used, a smaller $\chi$ will be required to achieve a connected path between the sides of the composite [89]. This is due to the fact that the long fibers are a long connected path already. This will result in a percolation threshold smaller than the Sher-Zallen invariant. The same is observed if plates or ribbons are used. For ME composites with conductive PM phase, spherical particles are required to increase the percolation threshold and to avoid leakage currents.

Another important factor that influences the percolation threshold is the size of the matrix precursor and the size of the dispersed phase particles [90]. For example, if the particulate composite is obtained by solid state sintering, particles of the PE phase will be mixed with particles of the PM phase. If those particles have the same size the percolation threshold will be closer to the Sher-Zallen invariant. If the particles of the PE phase are smaller than the 
particles of the PM phase, then the PE particles will cover the PM phase more easily, reducing the connection between PM particles, thus resulting on a higher percolation threshold. If the PM particles are smaller than the PE phase, then the percolation threshold will decrease. Because of this reason, the dispersed PM phase particles have to be larger than the PE phase particles used for the matrix of the composite.

One final effect that has to be considered is tunneling [86]. To conduct electrons between two conductive particles (separated by an insulator) direct contact is not required. There is a distance at which the electrons can "jump" between conductors, called the tunneling distance. This effect is particularly relevant when the particle size is in the order of the tunneling distance. As a result of tunneling the percolation threshold will decrease when small particles, in the nano meter size, are used.

The proposed model applies to non-percolated composites only. The Eshelby model predicts properties under the assumption that the dispersed phase is not in contact. Percolation has to be taken into account during fabrication and avoided as much as possible. Then, if the obtained composite is not percolated, the properties calculated with the proposed model will be valid.

\subsection{Validation}

The ME voltage coefficient calculated with the present model is compared with the results of the model reported by Nan et al., results can be seen in Figure 4.2. The properties of the PE and PM phase are listed in Table 4.1. The ME voltage coefficient is calculated using both, dilute approximation and Mori-Tanaka mean-field theory. In Figure 4.2, it can be seen that the slope of both approximations is similar for small PM volume fractions and the ME voltage coefficient is zero when $\chi \rightarrow 0$. Additionally, two different boundary conditions are used; clamped and unrestrained (as explained in Section 4.3). Results show that for Terfenol-D/PVDF composite, the ME voltage coefficient increases in the clamped condition. 


\begin{tabular}{c|c|c|c} 
Property & PVDF & Terfenol-D & Units \\
\hline$C_{11}$ & 4.84 & 82 & $10^{9} \mathrm{~N} / \mathrm{m}^{2}$ \\
\hline$C_{12}$ & 2.72 & 40 & $10^{9} \mathrm{~N} / \mathrm{m}^{2}$ \\
\hline$C_{13}$ & 2.22 & 40 & $10^{9} \mathrm{~N} / \mathrm{m}^{2}$ \\
\hline$C_{33}$ & 4.63 & 82 & $10^{9} \mathrm{~N} / \mathrm{m}^{2}$ \\
\hline$C_{44}$ & 0.526 & 38 & $10^{9} \mathrm{~N} / \mathrm{m}^{2}$ \\
\hline$\epsilon / \epsilon_{0}$ & 8 & 6 & \\
\hline$e_{31}$ & 0.0043 & 0 & $C / \mathrm{m}^{2}$ \\
\hline$e_{33}$ & -0.11 & 0 & $C / \mathrm{m}^{2}$ \\
\hline$e_{15}$ & -0.015 & 0 & $C / \mathrm{m}^{2}$ \\
\hline$\mu / \mu_{0}$ & 1 & 3 & \\
\hline$q_{13}$ & 0 & $-100^{a}$ & $N /(A m)$ \\
\hline$q_{33}$ & 0 & $700^{a}$ & $N /(A m)$ \\
\hline
\end{tabular}

Table 4.1: Material properties of PVDF (P(VDF-TrFE)) and Terfenol-D $\left(T b_{0.3} D y_{0.7} F e_{2}\right)$ from [91]. ${ }^{a}$ calculated from Figure 2.b (right) and elastic properties.

This can be attributed to the fact that more stress will be produced in PVDF by Terfenol-D if the composite is clamped at the boundaries. Nan et al. didn't report which boundary conditions where used for their calculations. From Equation (1) and (5) from [91], it can be inferred that unrestrained conditions were used.

The proposed model is further compared with the model of Harshe et al. [46], see Figure 4.3. The calculations for our model are made using the Mori-Tanaka approximation and unrestrained and clamped boundary conditions. In this case, the unrestrained configuration shows higher ME voltage coefficient than using clamped boundary conditions. This may be due to the fact that the elastic constant of both materials is similar resulting in higher stress in the PE phase for unrestrained conditions. The results calculated by Harshe et al. show the same trend but are three times higher in magnitude. This may be due to the fact that in their model they consider cubic particles, the shear stress is not taken into account, and the magnetic field distribution is not calculated (the model does not use $\mu$ of the PM and PE phase). Additionally, the results calculated by Nan et al. are shown in Figure 4.3. Nan et al. does not specify under which boundary conditions the ME voltage was calculated but it can be assumed that it was calculated in the unrestrained configuration. 


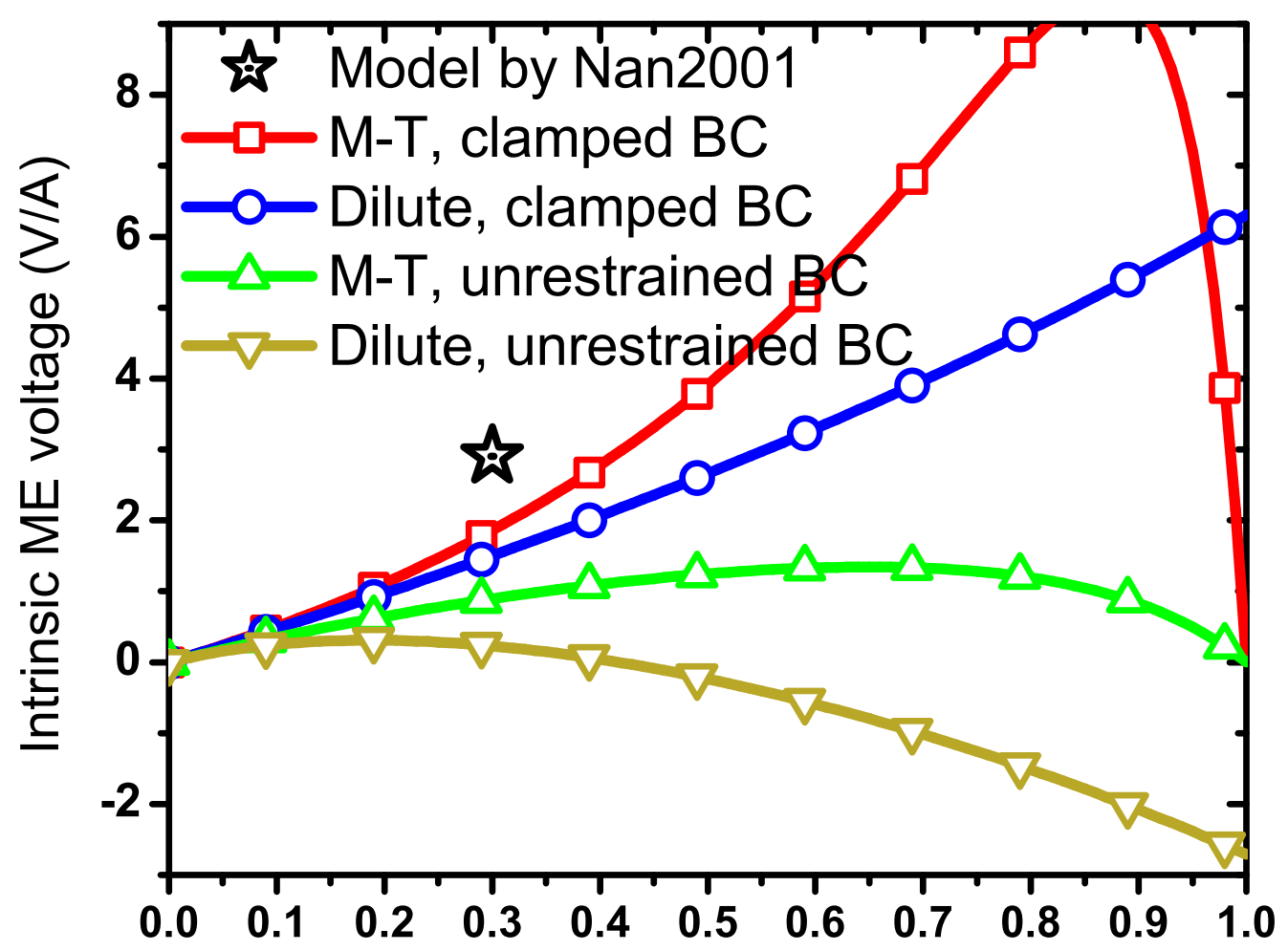

Figure 4.2: Calculated ME voltage coefficient of Terfenol-D/PVDF composite (Properties in Table 4.1) compared with the value reported by [91].

\begin{tabular}{c|c|c|c} 
Property & BTO & CFO & Units \\
\hline$C_{11}$ & 283 & 265 & $10^{9} \mathrm{~N} / \mathrm{m}^{2}$ \\
\hline$C_{12}$ & 186 & 152 & $10^{9} \mathrm{~N} / \mathrm{m}^{2}$ \\
\hline$C_{13}$ & 142 & 152 & $10^{9} \mathrm{~N} / \mathrm{m}^{2}$ \\
\hline$C_{33}$ & 178 & 265 & $10^{9} \mathrm{~N} / \mathrm{m}^{2}$ \\
\hline$C_{44}$ & 48 & 56 & $10^{9} \mathrm{~N} / \mathrm{m}^{2}$ \\
\hline$\epsilon / \epsilon_{0}$ & 1345 & 10 & \\
\hline$e_{31}$ & -9.69 & 0 & $C / \mathrm{m}^{2}$ \\
\hline$e_{33}$ & 11.7 & 0 & $C / \mathrm{m}^{2}$ \\
\hline$\mu / \mu_{0}$ & $8^{a}$ & $125^{a}$ & \\
\hline$q_{13}$ & 0 & 517 & $N /(A m)$ \\
\hline$q_{33}$ & 0 & 665 & $N /(A m)$ \\
\hline
\end{tabular}

Table 4.2: Material properties of BTO $\left(\mathrm{BaTiO}_{3}\right)$ and $\mathrm{CFO}\left(\mathrm{CoFe}_{2} \mathrm{O}_{4}\right)$ from [46]. Some properties are calculated using the relationship between coefficients [74]. ${ }^{a}$ obtained from [47]. 


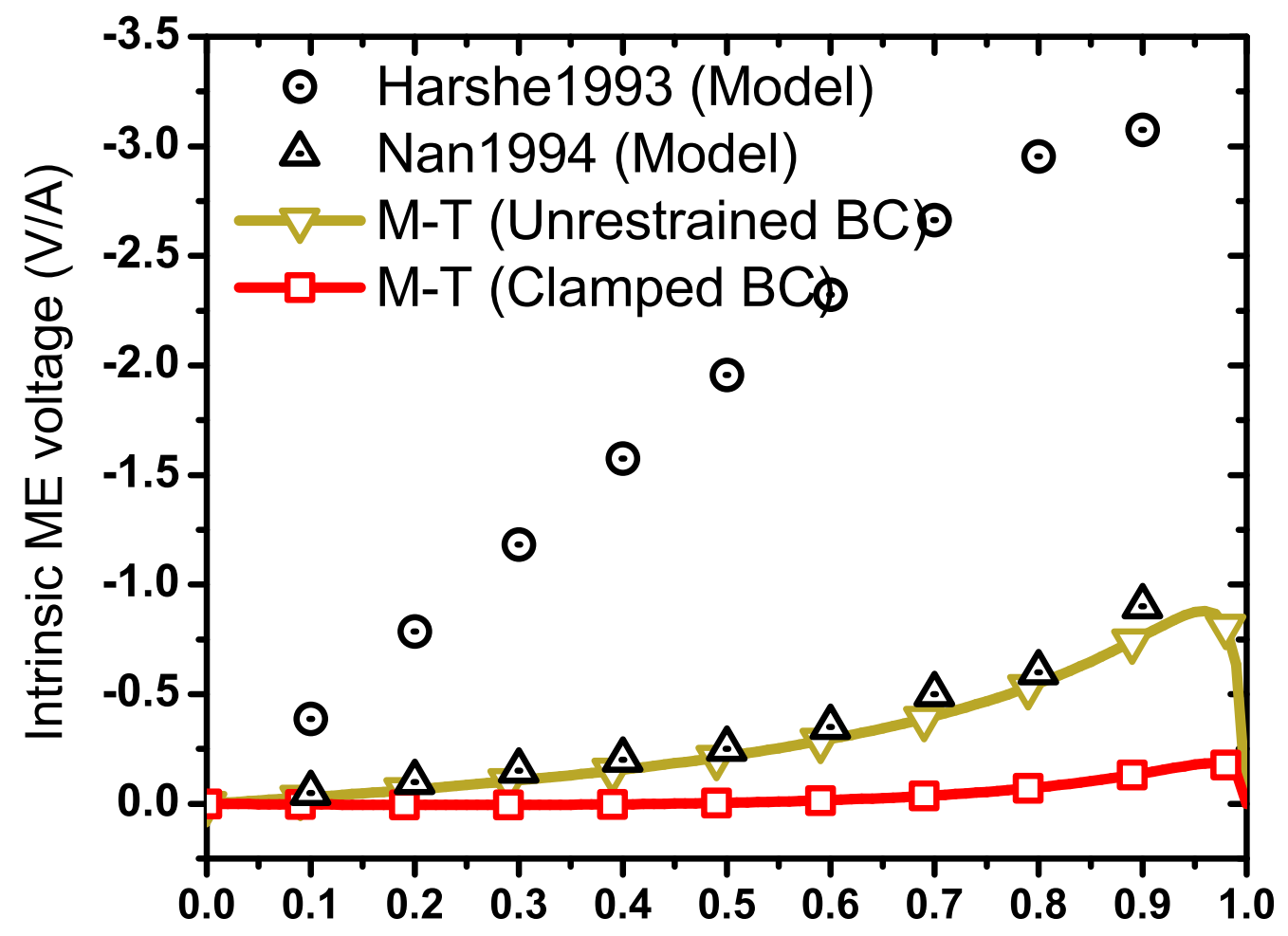

Figure 4.3: Calculated ME voltage coefficient of $\mathrm{CFO} / \mathrm{BTO}$ composite (Properties used in Table 4.2) compared with the value reported by [46] and [47]. 


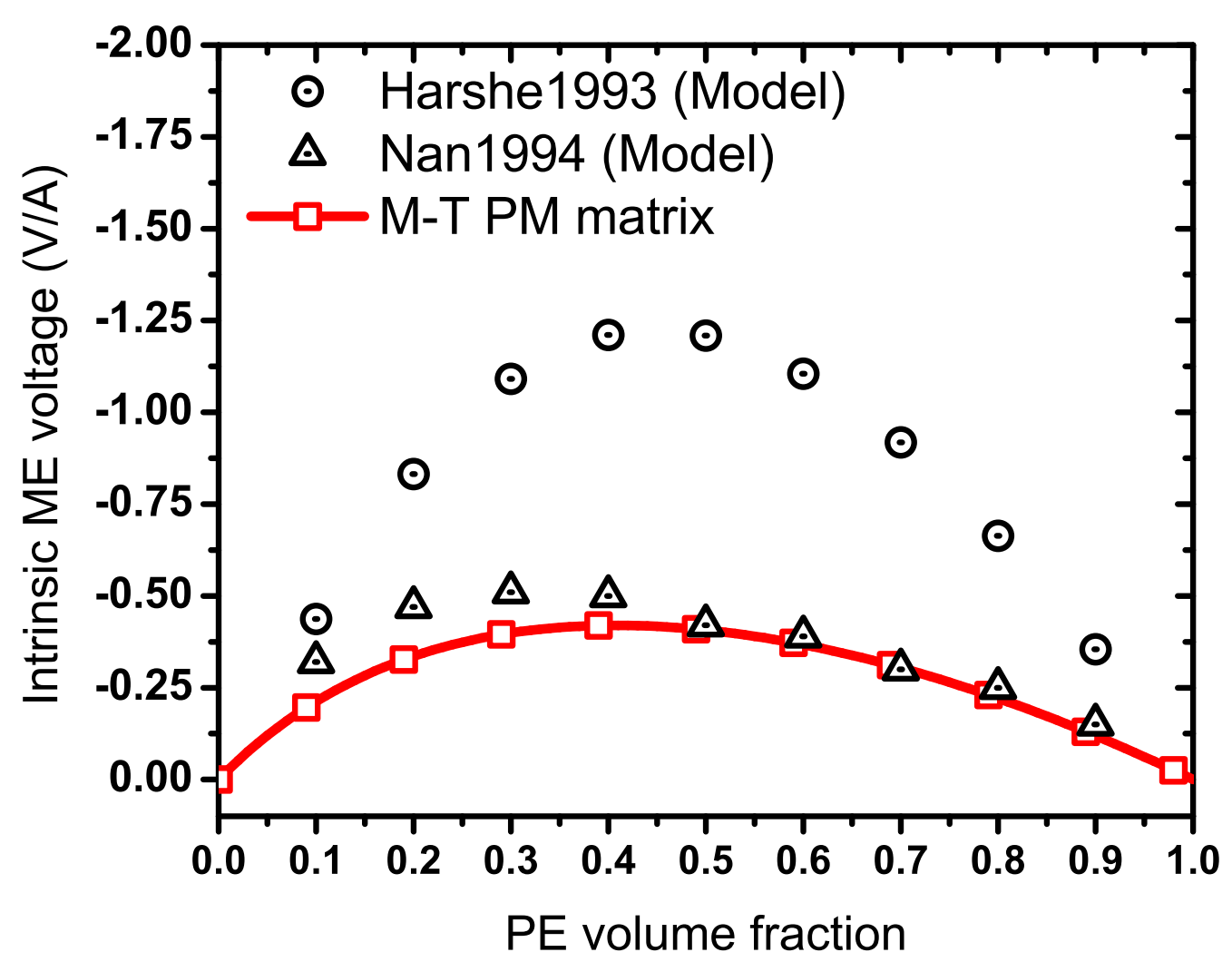

Figure 4.4: Calculated ME voltage coefficient of $\mathrm{CFO}$ (matrix)/BTO(dispersed phase) composite (Properties in Table 4.2) compared with the value reported by [46] and [47].

\subsubsection{Non-conductive PM matrix}

In some theoretical publications, the ME composites are assumed to be made out of PM matrix and the PE phase is supposed to be dispersed. To further validate the proposed model, this configuration (PM matrix with PE inclusions) was considered and compared with the results published by [46] and [47] (Figure 4.4). Here it can be seen that the proposed model has a similar trend to the values calculated by Nan et al. and Harshe et al.. The magnitude of the results calculated by [47] is the same and the values reported by [46] are over two times higher.

In practical applications the PM phase cannot be used as matrix because of the high electrical conductivity, as discussed in section 4.4. Using it as matrix will short circuit the PE 
particles, resulting in zero ME voltage coefficient. This is not observed in the results shown in Figure 4.4 because the conductive behavior is not taken into account when computing those results. However, the conductivity of the PM phase is accounted in the rest of this work by the approach proposed in Section 2, where the dielectric constant of the PM phase $\epsilon_{r}^{P M}$ is increased in order to reduce the electric field, as it is the case in a conductive material.

\subsubsection{Conductive PM matrix}

The use of the PM phase as a matrix cancels the ME effect due to the conductivity of the PM phase. Even though this cannot be obtained experimentally, previous models predict ME effect under these conditions. This is due to the fact that these predictions do not take into account the conductivity of the PM phase. The effect of the higher $\epsilon_{r}^{P M}$ to reduce the electric field inside the PM phase (as proposed in Section 2) is shown in Figure 4.5. The curve for $\epsilon_{r}^{P M}=10$ is the curve "M-T PM matrix" in Figure 4.4. As it is experimentally observed, the ME voltage coefficient is zero when a PM material is used as matrix. To model this, the PM dielectric constant has to be increased until the electric field in the PM phase is zero. In this case the value of the dielectric constant is $\epsilon_{r}^{P M}>10^{6}$. No difference can be seen between $\epsilon_{r}^{P M}=10^{6}$ and $\epsilon_{r}^{P M}=10^{9}$, because the electric field vanishes and further increment has no effect in the calculations.

\subsubsection{PE matrix with dispersed PM phase}

The approach used in Section 4.5.2 to account for the conductivity of the PM phase is used in this section for the practical case of PE matrix and further compared to experimental data. Without including the conductivity of the PM phase, when $\mathrm{CFO} / \mathrm{BTO}$ with unrestrained boundary conditions is compared with experimental data reported by Harshe et al., it can be seen that the predictions are over an order of magnitude higher [46]. If the conductivity of the PM phase is included by artificially increasing the dielectric constant, the ME voltage coefficient decreases as shown in Figure 4.6. $\epsilon_{r}^{P M}=10$ data is "M-T (Unrestrained BC)" in 


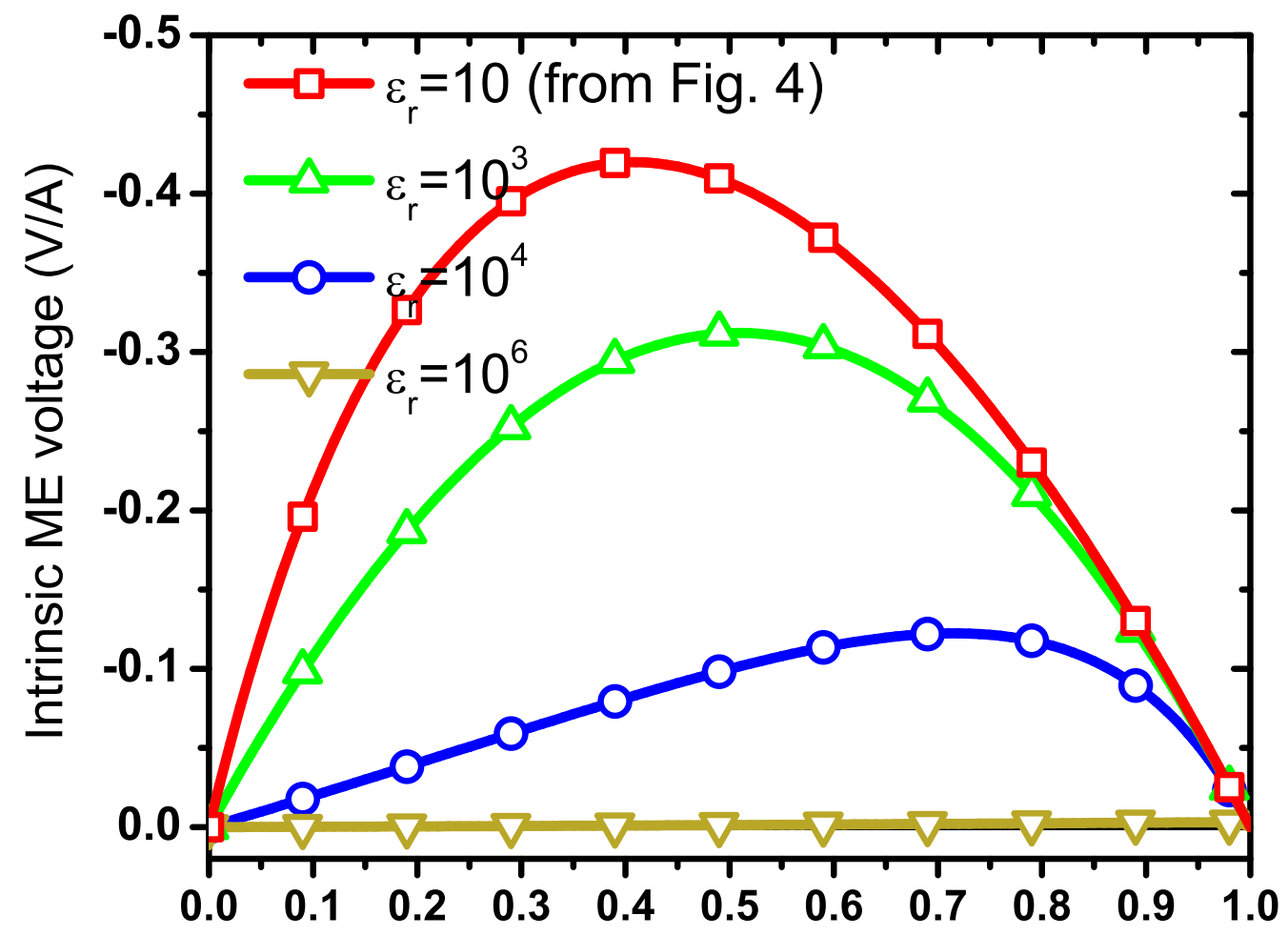

Figure 4.5: Calculated ME voltage coefficient of $\mathrm{CFO}$ (matrix)/BTO(dispersed phase) composite (Properties used in Table 4.2) for different values of the PM phase dielectric constant. The curve for $\epsilon_{r}=10$ is the curve "M-T PM matrix" in Figure 4.4. 
Figure 4.3 . In Figure 4.6, it can be seen that the predicted ME voltage coefficient changes sign. The reason behind the sign change is complex to identify and explain. For example, when a magnetic field is applied, the PM particles will expand. This expansion will produce compression on the sides and traction on the top of the matrix. The compression will produce a negative electric field and the traction a positive electric field. In this case the addition of these fields may result in a negative electric field. Then, when the dielectric constant of the PM phase is increased (representing the conductivity of the PM phase) the matrix on the sides of the particle will not be able to produce an electric field resulting in only a positive electric field from the matrix on top of the particle. In reality the model takes into account more effects such as shear stress and stresses in different directions, but a change in sign is reasonable and expected, as it was reported by Huang et al. and Nan et al. as well [42, 47].

Figure 4.7 shows a closer view of Figure 4.6 with the addition of calculations for matrix with $\epsilon_{r}^{P M}=10^{5}$ and $\epsilon_{r}^{P M}=10^{12}$. Here it can be seen that the proposed model approaches the experimental data when the dielectric constant of the PM phase is increased. There is no noticeable difference when the relative dielectric constant of the PM phase is increased over $10^{6}$ because the electric field in the PM phase is canceled already.

\subsection{Results}

The effect of the proposed model is further studied in the ME charge coefficient and the ME coupling factor. Both ME properties are calculated under the unrestrained condition. For design proposes this condition can be changed as explained in Section 4.3, but the objective of this work is not to design the optimum ME composite but to propose a model which has a good correlation with the experimental data. The effect of the PM volume fraction $\chi$ and the

dielectric permittivity of the PM phase $\epsilon_{r}^{P M}$ on the ME charge coefficient is shown in Figure 4.8. These results are similar to the ones observed for the ME voltage coefficient, showing consistency of the proposed approach. When $\epsilon_{r}^{P M}$ is increased to simulate the conductivity 


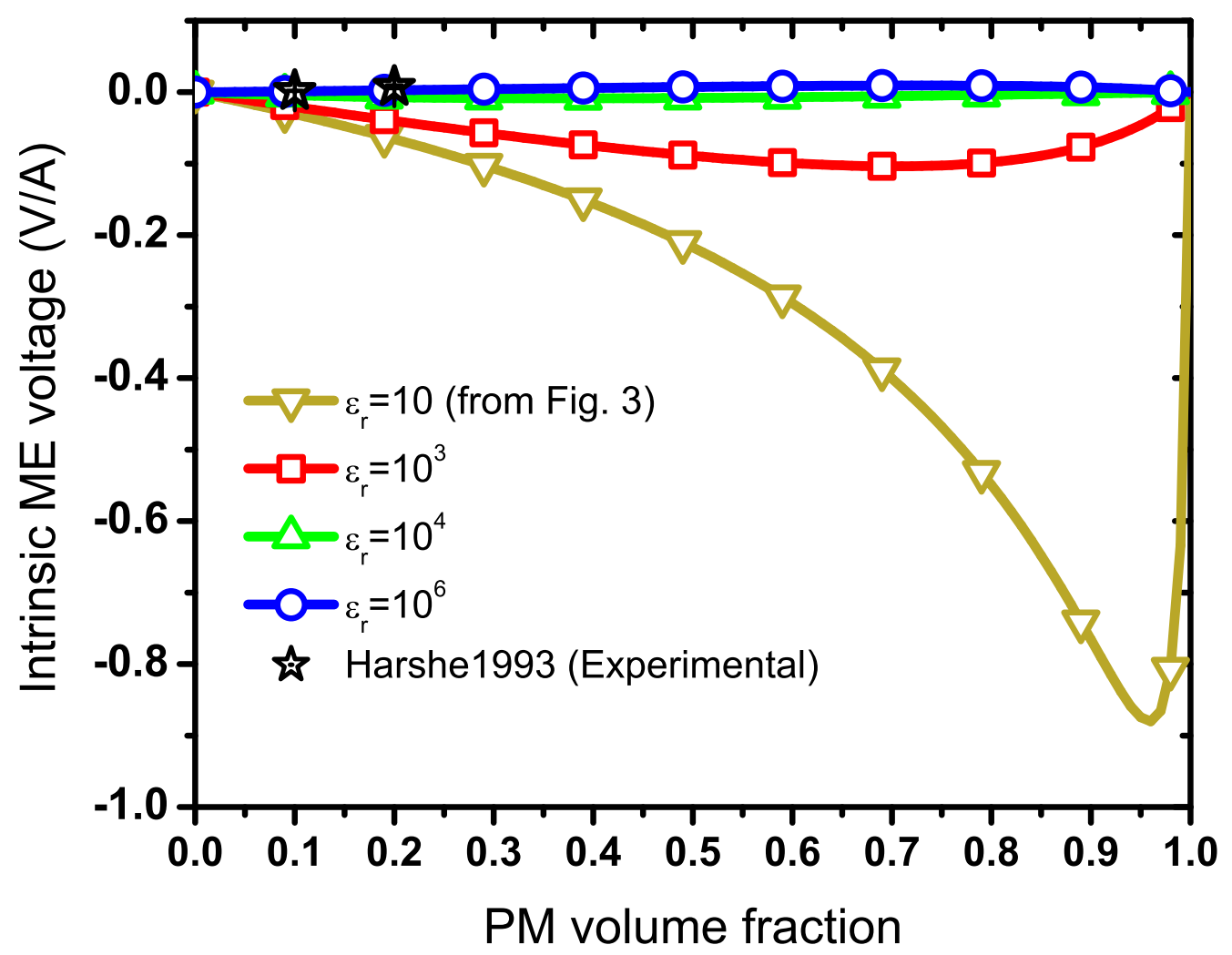

Figure 4.6: Calculated ME voltage coefficient using M-T approximation of $\mathrm{CFO}$ (dispersed phase)/BTO(matrix) composite (Properties in Table 4.2) for different values of the PM phase dielectric constant. Experimental data from [46]. $\epsilon=10$ data is "M-T (Unrestrained BC)" in Figure 4.3. 


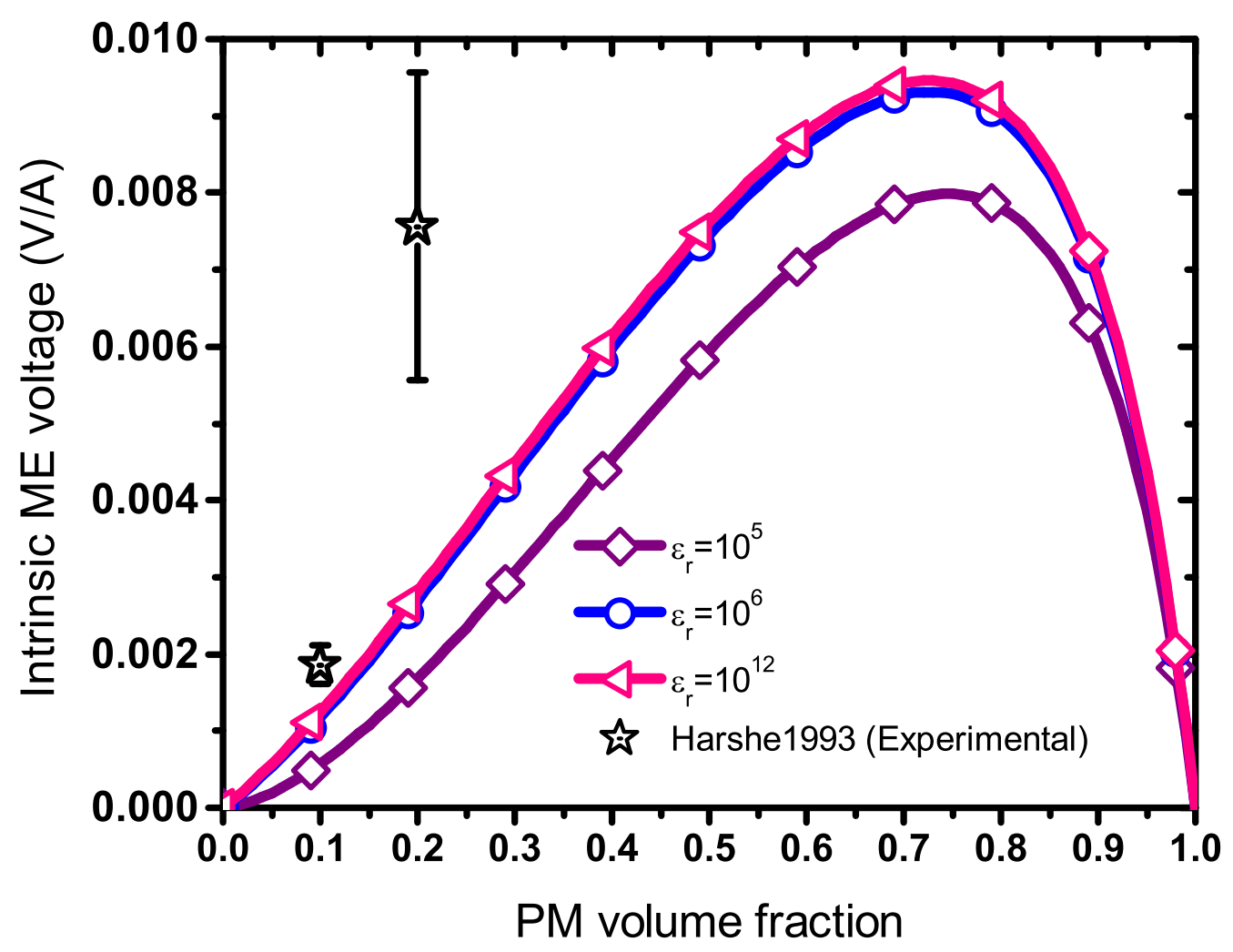

Figure 4.7: Zoom of figure 4.6. Calculated ME voltage coefficient of $\mathrm{CFO}$ (dispersed phase)/BTO(matrix) composite (Properties in Table 4.2) for different values of the PM phase dielectric constant. Experimental data from [46]. 


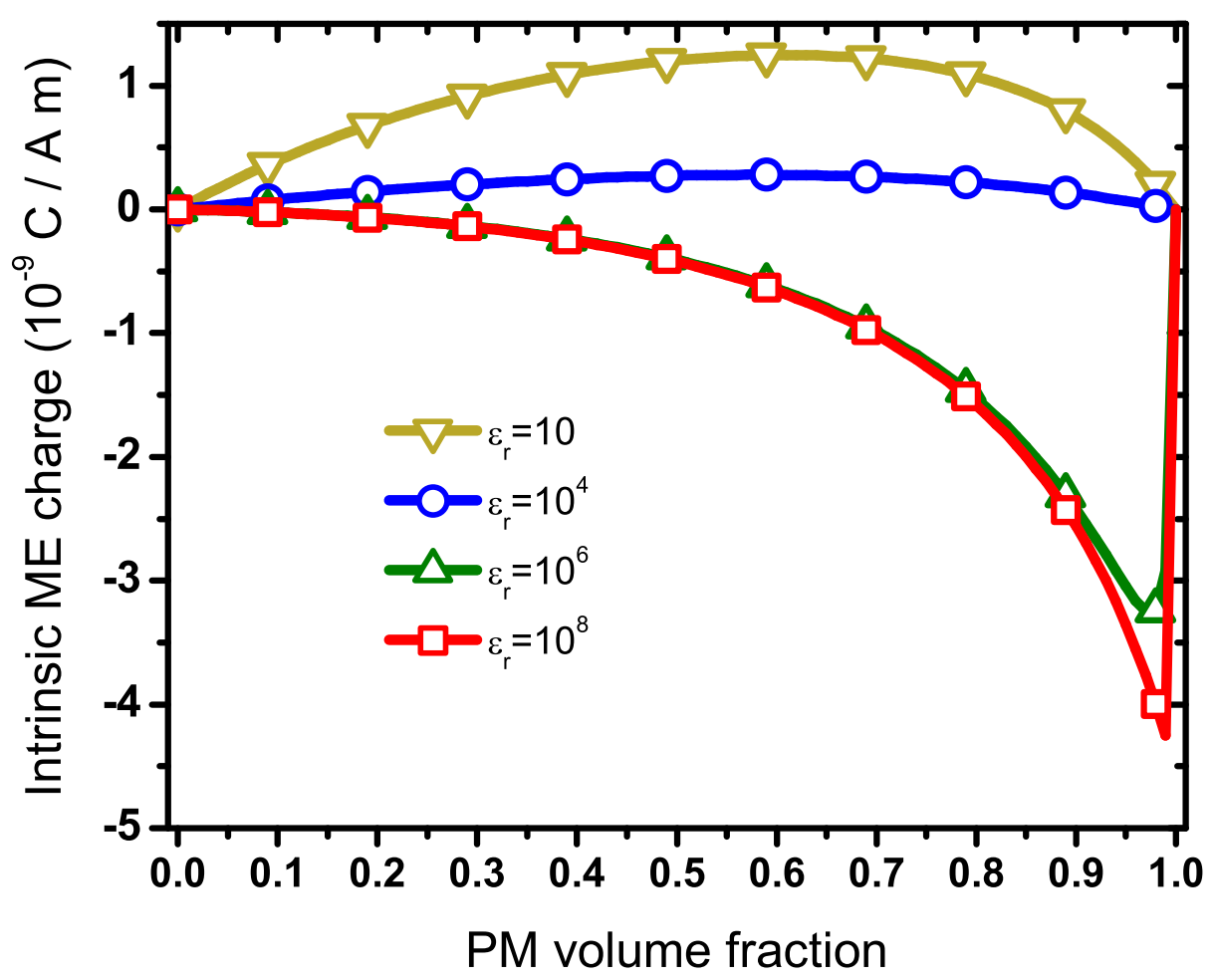

Figure 4.8: Calculated ME charge coefficient $\beta$ of $\mathrm{CFO}$ (dispersed phase)/BTO(matrix) composite (Properties in Table 4.2) for different values of the PM phase dielectric constant $\epsilon_{r}^{P M}$.

of the PM phase the $\beta$ decreases, changing sign. $\beta$ shows convergence when $\epsilon_{r}^{P M}$ is increased reaching a constant value for $\epsilon_{r}^{P M}>10^{6}$. The difference with the $\alpha$ and $\beta$ is that the magnitude of $\beta$ is bigger when the electric field inside the PM phase is zero.

The ME coupling factor $\kappa$ is a very important ME property for transducer applications like magnetic harvesters. The effect of the PM volume fraction $\chi$ and the dielectric permittivity of the PM phase $\epsilon_{r}^{P M}$ on the ME coupling factor is shown in Figure 4.9. Here it can be seen that when the electric field in the PM phase is reduced, by the increase of the $\epsilon_{r}^{P M}$, the $\kappa$ reduces almost an order of magnitude. The value of $\kappa$ shows convergence when the $\epsilon_{r}^{P M}$ is increased reaching a constant value for $\epsilon_{r}^{P M}>10^{6}$. 


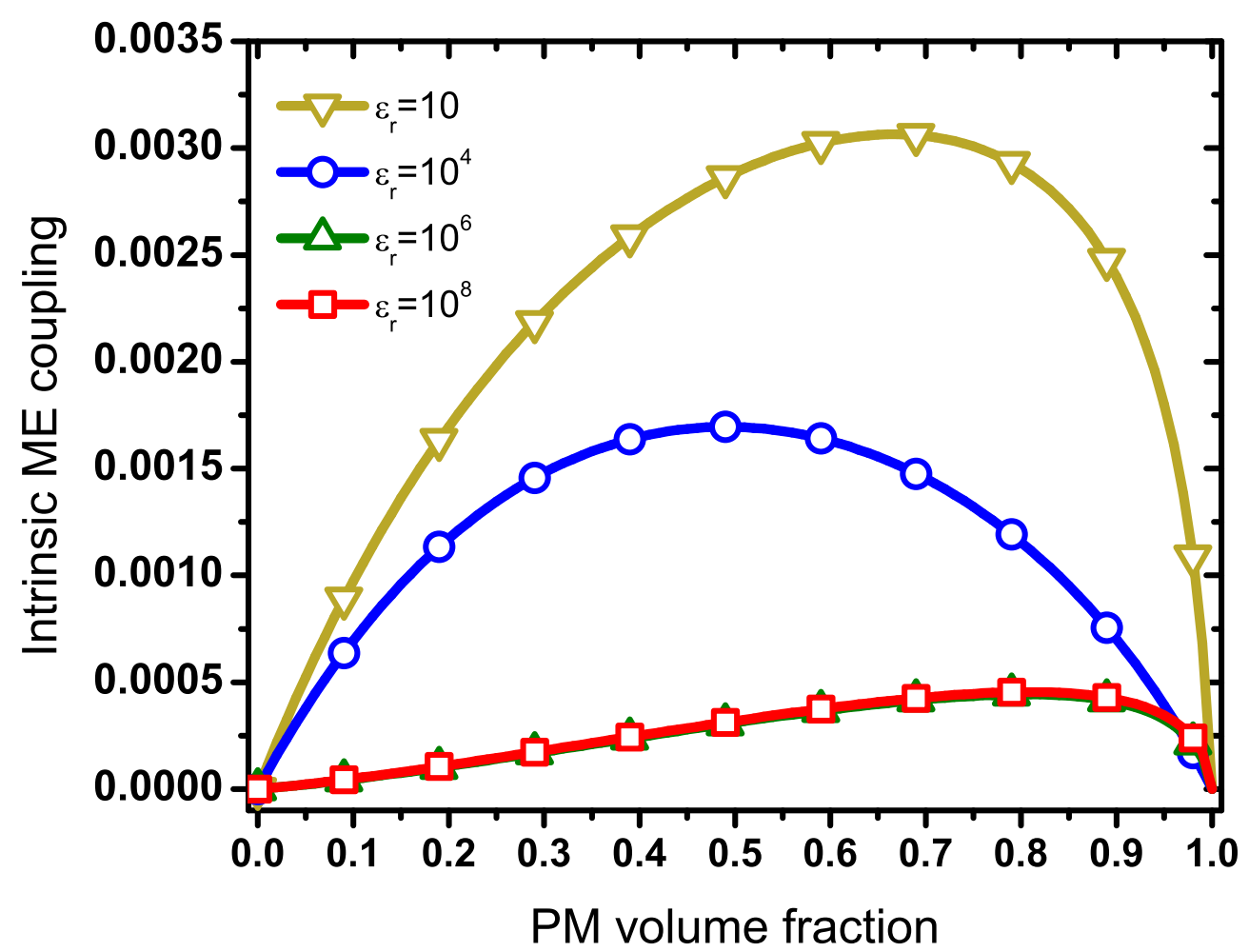

Figure 4.9: Calculated ME coupling factor $\kappa$ of CFO(dispersed phase)/BTO(matrix) composite (Properties in Table 4.2) for different values of the PM phase dielectric constant $\epsilon_{r}^{P M}$. 


\subsection{Conclusions}

An analytical solution is presented for predicting the magnetoelectric voltage, charge, and work conversion performance of particulate composites using Eshelby theory and MoriTanaka homogenization. The conductivity of the PM phase must be taken into account to achieve good correlation with experimental data. The model is able to reproduce the physical fact that a composite with PM matrix cannot deliver any output because the current is short circuited in the conductive matrix. Accounting for lack of conductivity, the model predictions compare well with other models in the literature that neglect conductivity. Including conductivity leads to good correlation with experimental data. The dilute approximation is satisfactory for very low volume fraction but the Mori-Tanaka is necessary for practical applications. 


\section{Chapter 5}

\section{Conclusions}

\subsection{Contributions}

In chapter 2, a new method is proposed to take into account the high conductivity of the PM phase. The method consists of artificially increasing the electrical permittivity of the PM phase. From the analysis of three composites made with three PM and three PE materials, analyzed for PM volume fraction continuously varying from zero to one, we conclude that setting the relative permittivity of the PM to $10^{9}$ is equivalent to a fully conducting PM material and thus a negligible electric field in the PM.

Artificially increasing the dielectric permittivity of the PM phase in the numerical calculation to simulate the high conductivity of said phase proved to be an expedient method for taking into account the conductivity of the PM material without having to introduce additional equations modeling electrical conductivity in the proposed model. In fact, as permittivity $\epsilon^{P M} \rightarrow \infty$, the ME charge coefficient vs. PM volume fraction plot becomes insensitivity to permittivity and the values predicted are close to experimental data for FeBSiC/PZN-PT (Fig. 2.6) for LT configuration. Also, the formulas become much simpler.

For the material systems analyzed, both ME voltage and charge coefficients are maximized when the PM volume fraction $\chi \rightarrow 1$ (in particular configurations). But such design is 
unrealistic because an infinitely thin layer of PE cannot generate any significant work. Thus, measuring the voltage or the current would be very difficult. In this regard, the newly developed ME coupling factor equations $(2.55,2.55,2.57)$ prove to be very useful in that they provide an indication of the work conversion that can be achieved.

For Terfenol-D/PZT-5H in TT configuration, a reduction of PM volume fraction from $100 \%$ to $90 \%$ results in a reduction of ME charge coefficient from an upper bound of $192 \times$ $10^{-9} \mathrm{CA}^{-1} \mathrm{~m}^{-1}$ down to $130 \times 10^{-9} \mathrm{CA}^{-1} \mathrm{~m}^{-1}$, while the ME coupling factor increases from zero to 0.17 . Note that a value of coupling factor $\kappa=1.0$, although practically unattainable, would indicate $100 \%$ work conversion.

In LL configuration, a reduction of PM volume fraction from $100 \%$ to $85 \%$ results in a reduction of ME voltage coefficient from an upper bound $\alpha=18 V A^{-1}$ down to $\alpha=$ $10.7 V A^{-1}$, while the ME coupling factor increases from zero to 0.32 . The formulas presented herein can be used to tune the volume fraction to achieve an acceptable compromise between ME charge, or voltage, and work conversion.

If work conversion needs to be maximized (for transducer applications), the optimum configuration is not TT but LL, at PM volume fraction $73 \%$, yielding a ME coupling factor (i.e., conversion) of 0.36 .

For accurate calculation, it is very important to use the appropriate elastic compliance of the material, which can vary significantly depending on the testing conditions. For example, the compliance in the polarization direction $S_{33}$ of PZT-5H changes from $20.7 \times 10^{-12}$ to $8.9 \times 10^{-12} \mathrm{~m}^{2} \mathrm{~N}^{-1}$ when it is measured at constant $E$ and constant $D$, respectively.

A trade off between voltage and PM volume fraction is evident for LSMO/PZT (Figure 2.3) yielding an optimum PM volume fraction $\chi=65 \%$ in TT configuration. Optimum values like this are dependent on material properties and configuration. Therefore, it is of significant interest for preliminary design and material selection to count with a complete set of formulas such as those proposed in this manuscript to be able to assess voltage, charge, and energy conversion for any of the four laminated configurations, as function of material 
and geometrical parameters.

In chapter 3 the extrinsic ME properties are proposed and calculated. The effects of demagnetization are drastic, not only in magnitude but also drastically changing material selection and volume fraction, both as function of the device's geometrical aspect ratio. PM materials with high magnetic permeability are very sensitive to demagnetizing effects and loose their comparative advantage even if they also posses high intrinsic coupling. There are two ways to minimize demagnetization: use a PM with low permeability $\mu$ or a PE with high compliance $S$. A composite with low PM permeability $\mu$ is better because the usable magnetic field $H_{3}$ in (3.15) is inversely proportional to $\mu$, so less $\mu$ means the performance is less affected by demagnetization. A composite with high PE compliance (e.g., PVDF) is best in LT and LL configurations because less PM is needed, and since only the PM is affected by demagnetization, the extrinsic performance is less affected.

The material combinations identified as having best intrinsic performance are replaced by different material combinations when extrinsic properties are calculated. Furthermore, the best material selection, optimum volume fraction, and even optimal configuration vary with the aspect ratio, thus requiring to include device geometry in the design process. The LL configuration yields maximum extrinsic ME voltage. For an aspect ratio of 10, ME devices based on Terfenol-D are predicted to have the highest extrinsic voltage coefficient due to its relatively small magnetic permeability $\mu_{33} / \mu_{0}=3$ (Table 3.2). In some cases, like for Metglas/PVDF, the decrease in the demagnetizing factor (due to smaller optimum PM volume fraction required to stretch the compliant PVDF) has a positive effect that more than compensates for the decrease in the intrinsic ME voltage resulting from using PVDF. However, different PM phases are required to achieve optimum performance at different values of aspect ratio.

The highest extrinsic $\alpha$ and $\kappa$ are predicted when the ME composite is polarized and magnetized in the longitudinal direction, justifying the more complicated fabrication required by longitudinal polarization, which requires an insulator to prevent charge leakage from the 
PE phase through the PM phase. Longitudinal magnetization yields higher extrinsic open circuit sensitivity due to the higher strain produced.

In chapter 4 , an analytical solution is presented for predicting the magnetoelectric voltage, charge, and work conversion performance of particulate composites using Eshelby theory and Mori-Tanaka homogenization. The conductivity of the PM phase must be taken into account to achieve good correlation with experimental data. The model is able to reproduce the physical fact that a composite with PM matrix cannot deliver any output because the current is short circuited in the conductive matrix. Accounting for lack of conductivity, the model predictions compare well with other models in the literature that neglect conductivity. Including conductivity leads to good correlation with experimental data. The dilute approximation is satisfactory for very low volume fraction but the Mori-Tanaka is necessary for practical applications.

\subsection{Future work}

In order to further validate the proposed models, a more systematic experimental procedure (than available data in literature) has to be made. The most important parameter are the properties of the PM and PE phase. These properties have to be measured for the particular polarization or magnetization and under the appropriate boundary conditions (i.e., when measuring the elastic properties the electrodes have to be in short or open circuit) because of the strong electro-mechanic coupling. As it was discussed in Chapter 2, the compliance changes from $20.7 \times 10^{-12}$ to $8.9 \times 10^{-12} \mathrm{~m}^{2} \mathrm{~N}^{-1}$ when it is measured at constant $E$ and constant $D$, respectively, for the case of PZT- $5 \mathrm{H}$. When the magnetic properties of the PM phase and the ME composite are measured, the corresponding demagnetizing effect has to be considered and accounted in the measurements as well.

To better understand the influence that the PM and PE phase properties have on the ME properties of the composite $(\alpha, \beta$ and $\kappa)$, a sensitivity study of all the inputs is required. 
This is helpful to elucidate which properties have higher impact in the ME output.

In the case of the particulate composite, the percolation of the PM phase has to be further studied experimentally. For example, the use of PE precursor with smaller particle size than the PM precursor to prevent from percolation and leakage of the generated charge. Also, a third phase can be incorporated to insulate the PM phase by coating it, and thus drastically reduce the conductivity and leakage currents.

The extrinsic ME properties of the particulate composite have to be calculated, accounting for demagnetizing effects, as described in Chapter 4. Furthermore, a systematic study like the one made for ME laminate composites (Chapter 3) has to be made for ME particulate composites. All the PM and PE material combinations and mechanical boundary conditions (bulk or thin film) have to be considered and the best candidates have to be selected for the corresponding applications and desired aspect ratio. 


\section{Bibliography}

[1] J.-P. Rivera, "On definitions, units, measurements, tensor forms of the linear magnetoelectric effect and on a new dynamic method applied to $\mathrm{Cr}-\mathrm{Cl}$ boracite," Ferroelectrics, vol. 161, no. 1, pp. 165-180, 1994.

[2] W. C. Rontgen, "Ueber die durch bewegung eines im homogenen electrischen felde befindlichen dielectricums hervorgerufene electrodynamische kraft," Annalen der Physik, vol. 271, no. 10, pp. 264-270, 1888.

[3] H. A. Wilson, "On the electric effect of rotating a dielectric in a magnetic field," Philosophical Transactions of the Royal Society of London. Series A, Containing Papers of a Mathematical or Physical Character, vol. 204, no. 372-386, pp. 121-137, 1905.

[4] P. Curie, E. translarion by J. Rosen, and P. Copie, "On symmetry in physical phenomena, symmetry of an electric field and of a magnetic field," Journal de physique, vol. 3, pp. $395-415,1894$.

[5] P. Debye, "Bemerkung zu einigen neuen versuchen ber einen magneto-elektrischen richteffekt.," The European physical journal. A, Hadrons and nuclei, vol. 36, pp. 300-301, 1926.

[6] G. T. Rado and V. J. Folen, "Observation of the magnetically induced magnetoelectric effect and evidence for antiferromagnetic domains," Physical review letters, vol. 7 , pp. 310-311, 1961. 
[7] J.-P. Rivera, "The linear magnetoelectric effect in $\mathrm{LiCoPO}_{4}$ revisited," Ferroelectrics, vol. 161, no. 1, pp. 147-164, 1994.

[8] M. Fiebig, "Revival of the magnetoelectric effect," Journal of Physics D: Applied Physics, vol. 38, no. 8, p. R123, 2005.

[9] B. Krichevtsov, V. Pavlov, and R. Pisarev, "Giant linear magnetoelectric effect in garnet ferrite films," JETP LETTERS, vol. 49, pp. 535-539, APR 251989.

[10] G. T. Rado, J. M. Ferrari, and W. G. Maisch, "Magnetoelectric susceptibility and magnetic symmetry of magnetoelectrically annealed $\mathrm{TbPO}_{4}$, " Physical review B, vol. 29, pp. 4041-4048, 1984.

[11] J. Ryu, S. Priya, K. Uchino, and H.-E. Kim, "Magnetoelectric effect in composites of magnetostrictive and piezoelectric materials," Journal of Electroceramics, vol. 8, no. 2, pp. 107-119, 2002.

[12] B. D. H. Tellegen, "The gyrator, a new electric network element.," Philips research reports, vol. 3, pp. 81 - 101, 1948.

[13] J. Boomgaard, D. R. Terrell, R. A. J. Born, and H. F. J. I. Giller, "An in situ grown eutectic magnetoelectric composite material," Journal of Materials Science, vol. 9, pp. 17051709, 1974 .

[14] A. M. J. G. Run, D. R. Terrell, and J. H. Scholing, "An in situ grown eutectic magnetoelectric composite material," Journal of Materials Science, vol. 9, pp. 1710-1714, 1974.

[15] M. Kothale, K. Patankar, S. Kadam, V. Mathe, A. Rao, and B. Chougule, "Dielectric behaviour and magnetoelectric effect in copper cobalt ferrite+barium lead titanate composites," Materials Chemistry and Physics, vol. 77, no. 3, pp. 691 - 696, 2003. 
[16] J. Y. Zhai, N. Cai, L. Liu, Y. H. Lin, and C. W. Nan, "Dielectric behavior and magnetoelectric properties of lead zirconate titanate/ Co -ferrite particulate composites," Materials Science and Engineering: B, vol. 99, no. 1, pp. 329 - 331, 2003.

[17] J. Ryu, A. V. Carazo, K. Uchino, and H.-E. Kim, "Piezoelectric and magnetoelectric properties of lead zirconate titanate/ $N i$-ferrite particulate composites," Journal of Electroceramics, vol. 7, pp. 17-24, 2001.

[18] V. Laletin and V. Pan'kov, "Influence of powder surface area on the magnetoelectric effect in barium lead zirconate titanate/nickel ferrite composite ceramics," Inorganic Materials, vol. 48, no. 1, pp. 87-92, 2012.

[19] R. Adnan Islam and S. Priya, "Progress in dual (piezoelectric-magnetostrictive) phase magnetoelectric sintered composites," 2012.

[20] C. A. Randall, N. Kim, J.-P. Kucera, W. Cao, and T. R. Shrout, "Intrinsic and extrinsic size effects in fine-grained morphotropic-phase-boundary lead zirconate titanate ceramics," Journal of the American Ceramic Society, vol. 81, no. 3, pp. 677-688, 1998.

[21] C.-W. Nan, N. Cai, L. Liu, J. Zhai, Y. Ye, and Y. Lin, "Coupled magnetic-electric properties and critical behavior in multiferroic particulate composites," Journal of Applied Physics, vol. 94, no. 9, pp. 5930-5936, 2003.

[22] J. Ryu, S. Priya, A. V. Carazo, K. Uchino, and H.-E. Kim, "Effect of the magnetostrictive layer on magnetoelectric properties in lead zirconate titanate/terfenol- D laminate composites," Journal of the American Ceramic Society, vol. 84, no. 12, pp. 2905-2908, 2001.

[23] J. Ryu, A. V. Carazo, K. Uchino, and H.-E. Kim, "Magnetoelectric properties in piezoelectric and magnetostrictive laminate composites," Japanese Journal of Applied Physics, vol. 40, no. Part 1, No. 8, pp. 4948-4951, 2001. 
[24] K. Mori and M. Wuttig, "Magnetoelectric coupling in terfenol D polyvinylidenedifluoride composites," Applied Physics Letters, vol. 81, no. 1, pp. 100-101, 2002.

[25] K. Uestuener, M. Katter, and W. Rodewald, "Dependence of the mean grain size and coercivity of sintered $N d-F e-B$ magnets on the initial powder particle size," in Magnetics Conference, 2006. INTERMAG 2006. IEEE International, p. 228, may 2006.

[26] N. Cai, C.-W. Nan, J. Zhai, and Y. Lin, "Large high-frequency magnetoelectric response in laminated composites of piezoelectric ceramics, rare-earth iron alloys and polymer," Applied Physics Letters, vol. 84, no. 18, pp. 3516-3518, 2004.

[27] G. Srinivasan, E. T. Rasmussen, and R. Hayes, "Magnetoelectric effects in ferrite-lead zirconate titanate layered composites: The influence of zinc substitution in ferrites," Phys. Rev. B, vol. 67, p. 014418, Jan 2003.

[28] M. I. Bichurin, D. A. Filippov, V. M. Petrov, V. M. Laletsin, N. Paddubnaya, and G. Srinivasan, "Resonance magnetoelectric effects in layered magnetostrictivepiezoelectric composites," Phys. Rev. B, vol. 68, p. 132408, Oct 2003.

[29] S. Dong, J. Zhai, F. Bai, J.-F. Li, and D. Viehland, "Push-pull mode magnetostrictive/piezoelectric laminate composite with an enhanced magnetoelectric voltage coefficient," Applied Physics Letters, vol. 87, no. 6, p. 062502, 2005.

[30] S. Dong, J. Zhai, J. Li, and D. Viehland, "Small de magnetic field response of magnetoelectric laminate composites," Applied Physics Letters, vol. 88, no. 8, p. 082907, 2006.

[31] S. Dong, J. Zhai, Z. Xing, J.-F. Li, and D. Viehland, "Extremely low frequency response of magnetoelectric multilayer composites," Applied Physics Letters, vol. 86, no. 10, p. 102901, 2005. 
[32] S. Dong, J. Zhai, J. F. Li, D. Viehland, and S. Priya, "Multimodal system for harvesting magnetic and mechanical energy," Applied Physics Letters, vol. 93, no. 10, p. 103511, 2008.

[33] S. Priya, J. Ryu, C.-S. Park, J. Oliver, J.-J. Choi, and D.-S. Park, "Piezoelectric and magnetoelectric thick films for fabricating power sources in wireless sensor nodes," Sensors, vol. 9, no. 8, pp. 6362-6384, 2009.

[34] S. P. Beeby, M. J. Tudor, and N. M. White, "Energy harvesting vibration sources for microsystems applications," Measurement Science and Technology, vol. 17, no. 12, p. R175, 2006.

[35] S. Priya, "Advances in energy harvesting using low profile piezoelectric transducers," Journal of Electroceramics, vol. 19, no. 1, pp. 165-182, 2007.

[36] R. Andosca, T. G. McDonald, V. Genova, S. Rosenberg, J. Keating, C. Benedixen, and J. Wu, "Experimental and theoretical studies on MEMS piezoelectric vibrational energy harvesters with mass loading," Sensors and Actuators A: Physical, vol. 178, no. 0, pp. 76 $-87,2012$.

[37] S. Dong, J. Zhai, J. Li, D. Viehland, and S. Priya, "High power density magnetoelectric energy harvester," in Applications of Ferroelectrics, 2008. ISAF 2008. 17th IEEE International Symposium on the, vol. 1, pp. 1 -2, feb. 2008.

[38] P. Li, Y. Wen, P. Liu, X. Li, and C. Jia, "A magnetoelectric energy harvester and management circuit for wireless sensor network," Sensors and Actuators A: Physical, vol. 157 , no. 1 , pp. $100-106,2010$.

[39] J. Yang, Y. Wen, P. Li, X. Dai, and M. Li, "A broadband vibration energy harvester using magnetoelectric transducer," in Sensors, 2010 IEEE, pp. 1905 -1909, nov. 2010. 
[40] S. D. Moss, J. E. McLeod, I. G. Powlesland, and S. C. Galea, "A bi-axial magnetoelectric vibration energy harvester," Sensors and Actuators A: Physical, vol. 175, no. 0, pp. 165 $-168,2012$.

[41] P. Li, Y. Wen, and L. Bian, "Enhanced magnetoelectric effects in composite of piezoelectric ceramics, rare-earth iron alloys, and ultrasonic horn," Applied Physics Letters, vol. 90, no. 2, p. 022503, 2007.

[42] J. Huang, R. O'Handley, and D. Bono, "New, high-sensitivity, hybrid magnetostrictive/electroactive magnetic field sensors," Proceedings of SPIE - The International Society for Optical Engineering, vol. 5050, pp. 229-237, 2003.

[43] X. Dai, Y. Wen, P. Li, J. Yang, and G. Zhang, "Modeling, characterization and fabrication of vibration energy harvester using Terfenol-D/PZT/Terfenol-D composite transducer," Sensors and Actuators A: Physical, vol. 156, no. 2, pp. 350 - 358, 2009.

[44] S. Roundy and Y. Zhang, "Toward self-tuning adaptive vibration based microgenerators," Proceedings of SPIE - The International Society for Optical Engineering, vol. 5649, no. PART 1, pp. 373-384, 2005.

[45] I. Sari, T. Balkan, and H. Kulah, "An electromagnetic micro power generator for wideband environmental vibrations," Sensors and Actuators, A: Physical, vol. 145-146, no. 12, pp. 405-413, 2008.

[46] G. Harshe, J. Dougherty, and R. Newnham, "Theoretical modelling of multilayer magnetoelectric composites," International journal of applied electromagnetics in materials, vol. 4, no. 2, pp. 145-159, 1993.

[47] C.-W. Nan, "Magnetoelectric effect in composites of piezoelectric and piezomagnetic phases," Physical Review B, vol. 50, pp. 6082-6088, 1994. 
[48] I. Osaretin and R. Rojas, "Theoretical model for the magnetoelectric effect in magnetostrictive/ piezoelectric composites," Physical Review B - Condensed Matter and Materials Physics, vol. 82, no. 17, 2010.

[49] M. Bichurin and V. Petrov, "Modeling of magnetoelectric interaction in magnetostrictive-piezoelectric composites," Advances in Condensed Matter Physics, 2012 .

[50] T. Sun, L. Sun, Z. Yong, H. Chan, and Y. Wang, "Estimation of the magnetoelectric coefficient of a piezoelectric- magnetostrictive composite via finite element analysis," Journal of Applied Physics, vol. 114, no. 2, 2013.

[51] K. Azoum, M. Besbes, F. Bouillault, and T. Ueno, "Modeling of magnetostrictive phenomena. application in magnetic force control," EPJ Applied Physics, vol. 36, no. 1, pp. $43-47,2006$.

[52] N. Galopin, X. Mininger, F. Bouillault, and L. Daniel, "Finite element modeling of magnetoelectric sensors," IEEE Transactions on Magnetics, vol. 44, no. 6, pp. 834-837, 2008.

[53] T. Nguyen, F. Bouillault, L. Daniel, and X. Mininger, "Finite element modeling of magnetic field sensors based on nonlinear magnetoelectric effect," Journal of Applied Physics, vol. 109, no. 8, 2011.

[54] X. Mininger, N. Galopin, Y. Dennemont, and F. Bouillault, "3d finite element model for magnetoelectric sensors," EPJ Applied Physics, vol. 52, no. 2, 2010.

[55] T. I. Muchenik and E. J. Barbero, "Charge, voltage, and work-conversion formulas for magnetoelectric laminated composites," Smart Mater. Struct., vol. 24, no. 025039, 2015.

[56] Z. Shi, C. Wang, X. Liu, and C. Nan, "A four-state memory cell based on magnetoelectric composite," Chinese Science Bulletin, vol. 53, no. 14, pp. 2135-2138, 2008. 
[57] A. Lakhtakia and T. G. Mackay, "Meet the metamaterials," Optics and Photonics, vol. 1, pp. 32-39, 2007.

[58] R. Kambale, D.-Y. Jeong, and J. Ryu, "Current status of magnetoelectric composite thin/thick films," Advances in Condensed Matter Physics, 2012.

[59] E. Barbero, Multifunctional Composites. CreateSpace, first ed., 2015.

[60] Wolfram Research, "Mathematica 9.0," tech. rep., Wolfram Research, Champaign, Illinois, 2013.

[61] S. Dong, J.-F. Li, and D. Viehland, "Magnetoelectric coupling, efficiency, and voltage gain effect in piezoelectric-piezomagnetic laminate composites," Journal of Materials Science, vol. 41, no. 1, pp. 97-106, 2006.

[62] K. Sun and Y. Kim, "Design of magnetoelectric multiferroic heterostructures by topology optimization," Journal of Physics D: Applied Physics, vol. 44, no. 18, 2011.

[63] C.-M. Chang and G. Carman, "Modeling shear lag and demagnetization effects in magneto-electric laminate composites," Physical Review B - Condensed Matter and Materials Physics, vol. 76, no. 13, 2007.

[64] C.-M. Chang and G. Carman, "Experimental evidence of end effects in magneto-electric laminate composites," Journal of Applied Physics, vol. 102, no. 12, 2007.

[65] E. Barbero, Finite Element Analysis of Composite Materials Using Abaqus. Composite Materials: Analysis and Design Series, Boca Raton, FL: Taylor and Francis, 2013.

[66] S. Dong, J. Zhai, J. Li, and D. Viehland, "Near-ideal magnetoelectricity in highpermeability magnetostrictive/ piezofiber laminates with a (2-1) connectivity," Applied Physics Letters, vol. 89, no. 25, 2006. 
[67] J. Na, T. Lee, and S. Park, "Effects of cation distribution on the magnetic and electrical properties of cobalt ferrite," IEEE Transactions on Magnetics, vol. 28, no. 5 pt 2, pp. 2433-2435, 1992.

[68] S. Dong, J. Zhai, Z. Xing, J. Li, and D. Viehland, "Giant magnetoelectric effect (under a de magnetic bias of $2 \mathrm{Oe}$ ) in laminate composites of $\mathrm{FeBSiC}$ alloy ribbons and $\mathrm{Pb}\left(\mathrm{Zn}_{1 / 3}, \mathrm{Nb}_{2 / 3}\right) \mathrm{O}_{3}-7$ \% PbTiO 3 fibers," Applied Physics Letters, vol. 91, no. 2, 2007.

[69] Web resource, 2014. http://barbero.cadec-online.com/papers/source/ME/ laminate/.

[70] R. Joseph and E. Schlomann, "Demagnetizing field in nonellipsoidal bodies," Journal of Applied Physics, vol. 36, no. 5, pp. 1579-1593, 1965.

[71] A. Aharoni, "Demagnetizing factors for rectangular ferromagnetic prisms," Journal of Applied Physics, vol. 83, no. 6, pp. 3432-3434, 1998.

[72] E. Quandt, S. Stein, and M. Wuttig, "Magnetic vector field sensor using magnetoelectric thin-film composites," IEEE Transactions on Magnetics, vol. 41, no. 10, pp. 3667-3669, 2005.

[73] D. Berlincourt and H. H. A. Krueger, "Properties of morgan electro ceramic ceramics," tech. rep., Morgan Electro Ceramics.

[74] C. Sherman and J. Butler, Transducers and Arrays for Underwater Sound. The Underwater Acoustics Series, Springer, 2007.

[75] T. I. Muchenik and E. J. Barbero, "Prediction of extrinsic charge, voltage, and workconversion factors for laminated magnetoelectric composites," Smart Materials and Structures, vol. 25, no. 1, p. 015006, 2016. 
[76] D. M. Esterly, Manufacturing of Poly(vinylidene fluoride) and Evaluation of its Mechanical Properties. PhD thesis, Virginia Polytechnic Institute and State University, 2002.

[77] B. D. Cullity and C. D. Graham, Experimental Methods, pp. 23-86. John Wiley \& Sons, Inc., 2008.

[78] T. I. Muchenik and E. J. Barbero, "Magnetoelectric composites," in Multifunctional Composites (E. J. Barbero, ed.), Charleston, SC: CreateSpace, 2015.

[79] J. Huang and W.-S. Kuo, "The analysis of piezoelectric/piezomagnetic composite materials containing ellipsoidal inclusions," Journal of Applied Physics, vol. 81, no. 3, pp. 1378$1386,1997$.

[80] M. Liu, X. Li, J. Lou, S. Zheng, K. Du, and N. X. Sun, "A modified sol-gel process for multiferroic nanocomposite films," Journal of Applied Physics, vol. 102, no. 8, p. 083911, 2007.

[81] C.-W. Nan, M. I. Bichurin, S. Dong, D. Viehland, and G. Srinivasan, "Multiferroic magnetoelectric composites: Historical perspective, status, and future directions," Journal of Applied Physics, vol. 103, no. 3, p. 031101, 2008.

[82] Y.-Z. Wang, "Influences of imperfect interface on effective magnetoelectric properties in multiferroic composites with elliptical fibers," Smart Materials and Structures, vol. 24, no. $4,2015$.

[83] M. Rawat and K. Yadav, "Electrical, magnetic and magnetodielectric properties in ferrite-ferroelectric particulate composites," Smart Materials and Structures, vol. 24, no. $4,2015$.

[84] H. Yang, G. Zhang, and Y. Lin, "Electrical, magnetic and magnetoelectric properties of laminated $0.65 \mathrm{BiFeO}_{3}-0.35 \mathrm{BaTiO}_{3} / \mathrm{BiY}_{2} \mathrm{Fe}_{5} \mathrm{O}_{12}$ composites," Smart Materials and Structures, vol. 24, no. 6, 2015. 
[85] J. D. Eshelby, "The elastic field outside an ellipsoidal inclusion," Proceedings of the Royal Society of London. Series A, Mathematical and Physical Sciences, vol. 252, no. 1271, pp. pp. 561-569, 1959 .

[86] T. Minoru, Electronic Composites. Cambridge University Press, 2005. Cambridge Books Online.

[87] E. Barbero, Finite Element Analysis of Composite Materials Using ANSYS. CRC Press, second ed., 2013.

[88] T. Mori and K. Tanaka, "Average stress in matrix and average elastic energy of materials with misfitting inclusions," Acta Metallurgica, vol. 21, no. 5, pp. 571-574, 1973.

[89] C.-W. Nan, Y. Shen, and J. Ma, "Physical properties of composites near percolation," Annual Review of Materials Research, vol. 40, pp. 131-151, 2010.

[90] R. Kusy, "Influence of particle size ratio on the continuity of aggregates," Journal of Applied Physics, vol. 48, no. 12, pp. 5301-5305, 1977.

[91] C. Nan, M. Li, X. Feng, and S. Yu, "Possible giant magnetoelectric effect of ferromagnetic rare-earth-iron-alloys-filled ferroelectric polymers," Applied Physics Letters, vol. 78, no. 17, pp. 2527-2529, 2001. 